\title{
Soft probes for bio-electrochemical imaging
}

\author{
THÈSE NO 8024 (2017) \\ PRÉSENTÉE LE 31 OCTOBRE 2017 \\ À LA FACULTÉ DES SCIENCES DE BASE \\ LABORATOIRE D'ÉLECTROCHIMIE PHYSIQUE ET ANALYTIQUE \\ PROGRAMME DOCTORAL EN CHIMIE ET GÉNIE CHIMIQUE \\ ÉCOLE POLYTECHNIQUE FÉDÉRALE DE LAUSANNE
}

POUR L'OBTENTION DU GRADE DE DOCTEUR ÈS SCIENCES

PAR

\section{Tzu-En LIN}

acceptée sur proposition du jury:

G. Wagnières, président du jury

Prof. H. Girault, Dr A. Lesch, directeurs de thèse

Prof. F. Kanoufi, rapporteur

Prof. S. Arbault, rapporteur

Prof. A. Hagfeldt, rapporteur

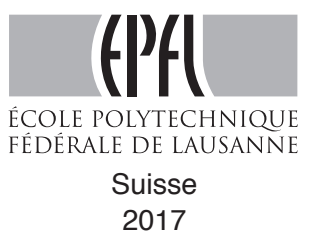





\section{Acknowledgement}

Firstly, I would like to express my sincere gratitude to my advisor Prof. Hubert Girault for the continuous support of my Ph.D study and for his creativity, motivation, and immense knowledge. His brilliant ideas inspired me to explore new fields of electrochemistry. His optimistic and generous attitude encouraged me to challenge difficult projects without fear. In addition, his humorous personality impressed everyone that science is not only dull equations but also full of interesting concepts related to our daily life. I could not have imagined having a better advisor for my Ph.D study.

Besides my supervisor, I would like to thank the rest of my thesis committee: Prof. Frédéric Kanoufi, Prof. Stéphane Arbault, Prof. Anders Hagfeldt, Georges Prof. Wagnières and Dr. Andreas Lesch, for their insightful comments and encouragement, as well as the questions they proposed during the defense which incented me to widen my research from various perspectives.

I am grateful to be under the co-supervision of Dr. Andreas Lesch and Fernando Cortés (for the first year), for assistance with the experiments I performed, and providing valuable comments that greatly improved the quality of my paper manuscripts and thesis for enlightening me the first glance of research.

Nevertheless, I am also thankful to the Prof. Gerald Fuller in Stanford University (USA) for his aspiring guidance during my visiting period in Stanford. A very special gratitude goes out to all down at Taiwanese Ministry of Education of scholarship and also Firmenich company for helping and providing the funding for the work and for the visiting of Stanford University. Also, I thank our lab secretary Patricia in particular for all the assistance she provided. I would like to express gratitude to all of the research collaborators for their help and support.

I thank my fellow labmates, especially Zhen, Alexandra, Yindi, Xiaoqin, Justyna, Milica, Victor, Natalia, Veronique, Shuai, Liang and Eugene, for the stimulating discussions, working heard in the gym to stay in shape, watching the aurora at $-20^{\circ} \mathrm{C}$ and for all the fun we have had in the last four years.

I also thank my parents for the unceasing encouragement, support and attention. Finally, I am grateful to God for the good health and wellbeing that were necessary to complete this thesis. 


\section{Abstract}

The aim of bioimaging is to visualize properties and processes of living objects or biological samples and extracts image-related information for revealing important physical structures or map the distribution of specific biomolecules in tissues for clinical purposes. However, current bioimaging methods which mainly rely on optical detection methods can suffer from optical interferences and thus bias the imaging results. Bio-electrochemical imaging with micrometer resolution represents a promising alternative tool since electrochemical signals, e.g. faradaic currents, depend exclusively on the redox reactions occurring at the sample surface and a sensing probe.

Scanning electrochemical microscopy (SECM) is a scanning probe technique that is composed of a micro- or nanoelectrode that can be positioned or scanned in close proximity to an interface. Faradaic current signals can be recorded due to the flux of redox active species between the sample and an amperometric SECM probe. SECM can be used to image the topography and reactivity of biological specimens for mapping localized biochemical activity. Although SECM has been applied to different biological systems, SECM studies of tissues are still under exploration. The reason is due to the shape and high roughness of such real samples and requires overcoming major drawbacks in conventional SECM instrumentation when scanning large, i.e. square centimeter sized, areas with irregular surface keeping a constant working distance.

This thesis aims to develop various reliable SECM bioimaging techniques for the study of the antioxidant defense system of fruit peels, distribution of biomarkers and nanomaterials in thin and thick animal samples, as well as human melanoma. Particularly the last is of major importance, because melanoma is the most lethal form of skin cancer striking thousands of people around the world. The survival rate depends on the stage of the cancer when it is diagnosed. Therefore, reliable methodologies for early diagnosis and unequivocal identification of cancer stages are of high relevance. One of the well-known melanoma biomarkers is «tyrosinase » which is the key enzyme involved in the biosynthesis of melanin and fruit maturation. Different tyrosinase SECM detection strategies were developed for the analysis of the spatial distribution of tyrosinase in melanoma as well as in banana samples. It is demonstrated in this thesis that SECM could improve the diagnosis and understanding of different melanoma stages based on highly resolved maps of the tyrosinase distribution while being immune 
against optical interferences, e.g. from the presence of melanin in the skin samples. Spider probe composed of eight independent microelectrodes was developed in this thesis and allowed the large area scanning in contact mode on thin and thick animal tissues. The redox active proteins inside the entire mouse heart were imaged with an SECM system for the first time by spider probe.

In addition, the distributions of injected conductive graphene nanoribbons (GONRs) for drug delivery were studied by Soft-Probe-SECM. Through the mapping of feedback mode currents over conductive GONRs, the GONRs were found concentrated inside lobules, which are hexagonal microstructures in the liver. Finally, this thesis describes a non-invasive electrochemical strategy for mapping the antioxidant (AO) activity of apple peels using Soft-Probe-SECM. The global AO activity in the apple peel including lenticels and regions with artificially degraded AOs were mapped.

In the future, further functionalization of the soft probes by integrating microfluidics could allow the delivery of anti-cancer drugs at the tip of the probe and the extraction of species locally generated by stimulated living cells for cancer research.

\section{Keywords}

scanning electrochemical microscopy, microelectrodes, soft probe, spider probe, tyrosinase, melanoma, graphene oxide nanoribbon, thick tissue, contact mode scanning, feedback mode, generation collection mode. 


\section{Résumé}

Le but de la bioimagerie est de visualiser les propriétés et les processus d'objets vivants ou d'échantillons biologiques et d'extraire des informations liées à l'image pour révéler des structures physiques importantes ou cartographier des biomolécules spécifiques à des fins cliniques. Cependant, les méthodes actuelles de bioimagerie qui reposent principalement sur les méthodes optiques peuvent subir des interférences optiques et donc altérer le résultat de l'imagerie. L'imagerie bio-électrochimique représente un outil alternatif prometteur car son signal dépend exclusivement des réactions rédox qui se produisent à la surface de la sonde.

La microscopie électrochimique à balayage (SECM) est une technique de sonde de balayage composée d'une micro ou d'une nanoélectrode qui peut être positionnée ou scannée à proximité d'une interface. Les signaux de courant Faradaic peuvent être enregistrés en raison du flux d'espèces actives rédox entre l'échantillon et une sonde SECM ampérométrique. SECM peut être utilisé pour imaginer la topographie et la réactivité des spécimens biologiques pour la cartographie de l'activité biochimique localisée. Bien que SECM ait été appliqué à différents systèmes biologiques, les études SECM sur les tissus sont toujours en cours d'exploration. La raison en est que l'étude des échantillons réels nécessite de remédier à plusieurs inconvénients de l'instrumentation SECM conventionnelle lors du balayage de surfaces grandes et irrégulières.

Cette thèse vise à développer diverses techniques de bioimagerie SECM pour étudier des échantillons de fruits, des échantillons d'animaux épais et minces, ainsi que le mélanome humain, la forme la plus létale de cancer de la peau qui frappe des milliers de personnes dans le monde. Le taux de survie dépend du stade du cancer lorsqu'il est diagnostiqué. Par conséquent, des méthodologies fiables pour le diagnostic précoce et l'identification sans équivoque des stades du cancer sont très pertinentes. L'un des biomarqueurs de mélanome bien connus est la «tyrosinase» qui est l'enzyme clé impliquée dans la biosynthèse de la mélanine et la maturation des fruits. Différentes stratégies de détection de la tyrosinase SECM ont été développées pour l'analyse de la distribution spatiale de la tyrosinase dans le mélanome et sa présence dans des échantillons de bananes. SECM peut surmonter l'interférence optique de la mélanine et améliorer le diagnostic et la compréhension des différentes étapes du mélanome en 
utilisant différents modèles de distribution de la tyrosinase. La sonde spider composée de huit microélectrodes indépendantes développée dans cette thèse a permis le balayage sur du grande surface de tissus animaux minces et épais en mode contact. Les protéines redox actives à l'intérieur du cœur entier de la souris ont été imagées pour la première fois par la sonde spider.

De plus, les distributions de nanorubans conducteurs à graphène injecté (GONR) pour la délivrance de médicaments ont été étudiées par Soft-Probe-SECM. Grâce à la cartographie des courants de la régénération du médiateur redox des GONR, les GONR ont été trouvés concentrés dans les lobules, qui sont des microstructures hexagonales dans le foie. Enfin, cette thèse décrit une stratégie électrochimique non invasive pour cartographier l'activité antioxydante (AO) des pelures de pomme en utilisant SoftProbe-SECM. L'activité AO globale dans la pelures de pomme, y compris les lenticelles et les régions avec AOs artificiellement dégradé, a été cartographiée.

À l'avenir, une fonctionnalisation accrue des sondes douces devrait conduire à l'intégration de la microfluidique permettant la délivrance d'un médicament anti-cancer donné à la pointe de la sonde et l'extraction des espèces générées par l'interaction avec les échantillons tels que les cellules vivantes.

\section{Mots-clés}

scanning electrochemical microscopy, microelectrodes, soft probe, spider probe, tyrosinase, melanoma, graphene oxide nanoribbon, thick tissue, contact mode scanning, feedback mode, generation collection mode. 


\section{List of abbreviations}

\begin{tabular}{|c|c|}
\hline $2 \mathrm{D}$ & Two dimensional \\
\hline $3 \mathrm{D}$ & Three dimensional \\
\hline BCIP & 5-bromo-4-chloro-3-indoly phosphate \\
\hline $\mathrm{Ab}$ & Antibody \\
\hline $\mathrm{AC}$ & Alternating current \\
\hline AFM & Atomic force microscopy \\
\hline ALP & Alkaline phosphatase \\
\hline $\mathrm{AJCC}$ & American joint Committee on Cancer \\
\hline $\mathrm{AO}$ & Antioxidant \\
\hline AP & alkaline phosphatase \\
\hline BSA & Bovine serum ablbumin \\
\hline $\mathrm{CA}$ & Chronoamperometry \\
\hline $\mathrm{CE}$ & Counter electrode \\
\hline $\mathrm{CGH}$ & Comparative genomic hybridization \\
\hline CNT & Carbon nanotube \\
\hline $\mathrm{CV}$ & Cyclic voltammograms \\
\hline $\mathrm{DAB}$ & 3,3'-diaminobenzidine \\
\hline DHI & 5,6-dihydroxyindole \\
\hline DOPA & L-3,4-dihydroxyphenylalanine \\
\hline DPV & Differential pulse voltammetry \\
\hline $\mathrm{DOX}$ & Doxorubicin \\
\hline EGFR & Epidermal growth factor receptor \\
\hline ECSTM & Electrochemical scanning tunneling microscopy \\
\hline ESTASI & Electrostatic spray ionization \\
\hline $\mathrm{FcMeOH}$ & Ferrocene carboxylic acid \\
\hline FFPE & Formalin-fixed paraffin-embedded \\
\hline FISH & Fluorescence in situ hybridization \\
\hline FRAP & Ferric ion reducing antioxidant power \\
\hline GO & Graphene oxide \\
\hline GONR & Graphene oxide nanoribbon \\
\hline GQD & Graphene quantum dot \\
\hline GSH & Glutathione \\
\hline $\mathrm{Hb}$ & Hemoglobin \\
\hline HRP & Horse radish peroxidase \\
\hline IFE & Isoelectrofocusing \\
\hline $\mathrm{IHC}$ & Immunohistochemistry \\
\hline
\end{tabular}




\begin{tabular}{|c|c|}
\hline LOD & Limit of detection \\
\hline LOQ & Limit of quantification \\
\hline MC1R & Melanocortin 1 receptor antigen \\
\hline $\mathrm{mCi}$ & millicurie \\
\hline MIRA & Microscopic image rapid analysis \\
\hline MMD & Multi-metal-deposition \\
\hline MNP & Magnetic nanoparticle \\
\hline MS & Mass spectrometry \\
\hline MSI & Mass spectrometry imaging \\
\hline MWCNT & Multiwall carbon nanotubes \\
\hline NBT & Nitroblue tetrazolium \\
\hline $\mathrm{NE}$ & Nanoelectrode \\
\hline $\mathrm{NF}$ & Negative feedback \\
\hline NFR & Nuclear fast red \\
\hline $\mathrm{O}$ & Oxidized form of the redox active species \\
\hline ORR & Oxygen reduction reaction \\
\hline PAP & P-aminophenol \\
\hline PAPP & P-aminophenol phosphate \\
\hline PBS & Phosphate buffer saline \\
\hline PET & Polyethylene terephathalate \\
\hline \multirow{2}{*}{$\mathrm{PET} / \mathrm{CT}$} & Positron Emission \\
\hline & Tomography \\
\hline PFA & Paraformaldehyde \\
\hline PEG & Polyethylene glycol \\
\hline PI & Polyimide \\
\hline PTFE & Polytetrafluoroethylene \\
\hline PVDF & Polyvinyldifluoride \\
\hline QRE & Quasi-reference electrode \\
\hline $\mathrm{R}$ & Reduced form of the redox active species \\
\hline $\mathrm{RE}$ & Reference electrode \\
\hline RES & Reticuloendothelial system \\
\hline rGO & Reduced graphene oxide \\
\hline RGP & Radial growth phase \\
\hline ROS & Reactive oxygen species \\
\hline RT & Room temperature \\
\hline SDS-PAGE & $\begin{array}{l}\text { Sodium dodecyl sulfate polyacrylamide gel } \\
\text { electrophoresis }\end{array}$ \\
\hline SECM & Scanning electrochemical microscopy \\
\hline
\end{tabular}




\begin{tabular}{ll}
\hline SG/TC & Substrate generation/tip collection \\
SICM & Scanning ion-conductive microscopy \\
SPM & Scanning probe microscopy \\
T & Tip \\
TG/SG & Tip generation/substrate collection \\
TMA & Tissue micro-arrays \\
TMB & 3,3 ',5,5'-tetramethylbenzidine \\
TNM & Tumor, node, metastasis system \\
TyR & Tyrosinase \\
UME & Ultramicroelectrode \\
UV & Ultraviolet \\
VGP & Vertical growth phase \\
WE & Working electrode \\
$\mu C P$ & Microcontact printing \\
\hline
\end{tabular}




\section{List of symbols}

\begin{tabular}{|c|c|c|}
\hline Symbol & Meaning Units & Units \\
\hline$c$ & Concentration & $\mathrm{mol} \mathrm{m}^{-3}$ \\
\hline$c_{0}$ & Maximum concentration & $\mathrm{mol} \mathrm{m}^{-3}$ \\
\hline$c_{\text {Bulk }}$ & Bulk concentration & $\mathrm{mol} \mathrm{m}^{-3}$ \\
\hline$D$ & Diffusion coefficient & $\mathrm{m}^{2} \mathrm{~s}^{-1}$ \\
\hline$d$ & Probe-substrate distance & $\mu \mathrm{m}$ \\
\hline$E_{\mathrm{T}}$ & Probe potential & $\mathrm{V}$ \\
\hline $\mathrm{F}$ & Faraday constant & $\mathrm{C}$ \\
\hline$h \mathrm{p}$ & $\begin{array}{l}\text { Real probe-substrate } \\
\text { distance for plastic } \\
\text { microelectrode }\end{array}$ & $\mu \mathrm{m}$ \\
\hline$i$ & Current & $\mathrm{nA}$ \\
\hline$I$ & Normalized current & None \\
\hline$i_{\mathrm{T}}$ & $\begin{array}{l}\text { Steady-state diffusion } \\
\text { current recorded at each } \\
\text { point }\end{array}$ & $\mathrm{nA}$ \\
\hline$i_{\mathrm{T}, \infty}$ & $\begin{array}{l}\text { Steady-state diffusion } \\
\text { current recorded at the } \\
\text { solution bulk }\end{array}$ & $\mathrm{nA}$ \\
\hline$i_{\mathrm{T}, \mathrm{offset}}$ & $\begin{array}{l}\text { Correction values vector } \\
\text { for the current offset }\end{array}$ & $\mathrm{nA}$ \\
\hline$k$ & $\begin{array}{l}\text { Heterogeneous kinetic } \\
\text { constant }\end{array}$ & $\mathrm{m} \mathrm{s}^{-1}$ \\
\hline$L$ & $\begin{array}{l}\text { Normalized probe- } \\
\text { substrate distance }\end{array}$ & None \\
\hline$n$ & $\begin{array}{l}\text { Number of transfer } \\
\text { electrons }\end{array}$ & None \\
\hline$R$ & Resistance & $\Omega$ \\
\hline$R G$ & $\begin{array}{l}\text { The ratio between } r_{\text {glass }} \\
\text { and } r_{\mathrm{T}}\end{array}$ & None \\
\hline$r_{\text {glass }}$ & $\begin{array}{l}\text { Insulating glass sheath } \\
\text { radius }\end{array}$ & $\mu \mathrm{m}$ \\
\hline$r_{\mathrm{T}}$ & Tip radius & $\mu \mathrm{m}$ \\
\hline $\mathrm{s}$ & $\begin{array}{l}\text { Correction values vector } \\
\text { for the dimensionless scale } \\
\text { factor }\end{array}$ & None \\
\hline
\end{tabular}




\begin{tabular}{lll}
\hline$t$ & Time & $\mathrm{s}$ \\
$v_{\mathrm{T}}$ & Translation rate & $\mu \mathrm{s}^{-1}$ \\
$\Lambda$ & Apparent kinetic constant & \\
\hline
\end{tabular}




\section{Content}

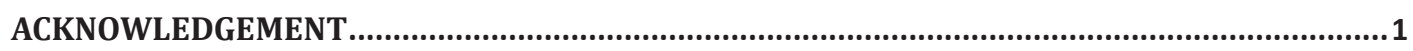

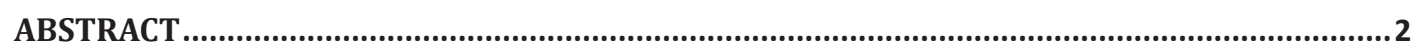

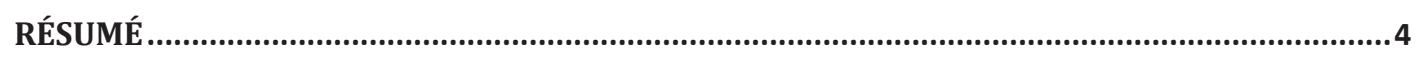

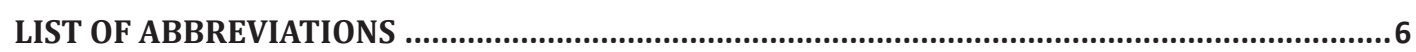

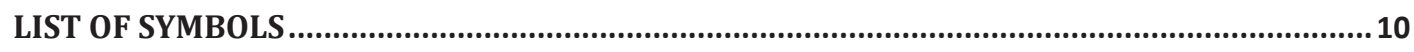

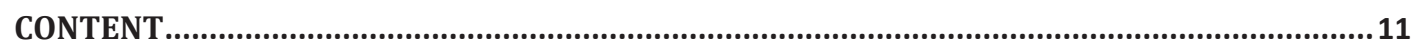

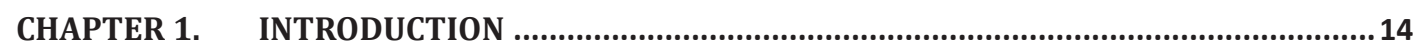

1.1 THEORY AND PRINCIPLES OF SCANNING ELECTROCHEMICAL MICROSCOPY (SECM) .............................. 14

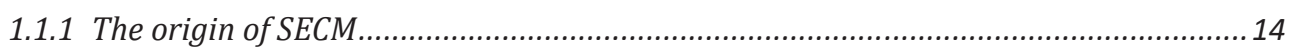

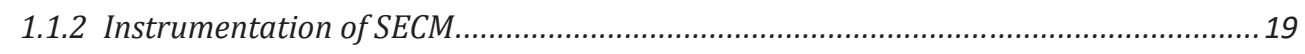

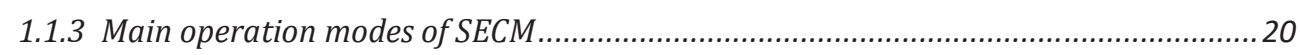

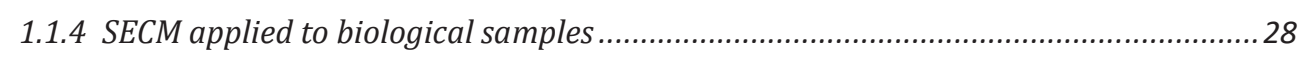

1.1.5 Limitations of SECM for real samples and approaches to overcome them .................29

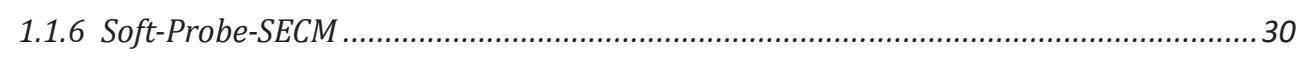

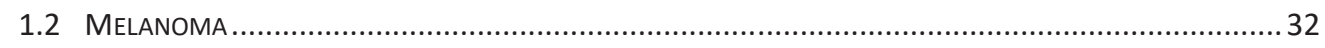

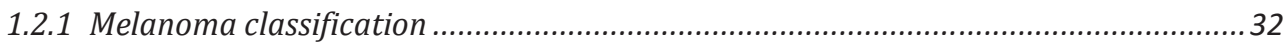

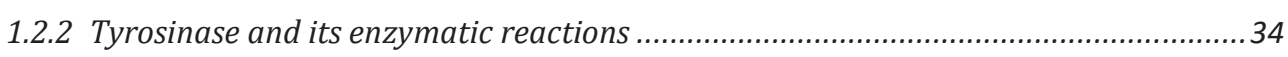

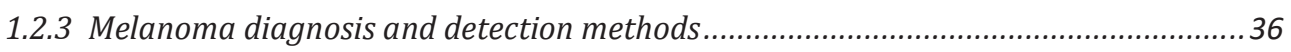

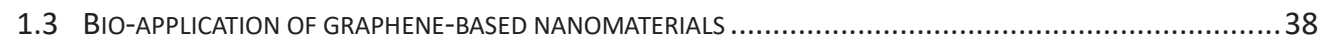

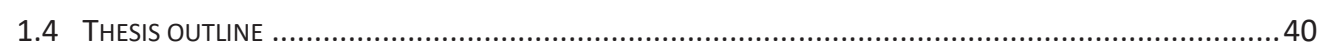

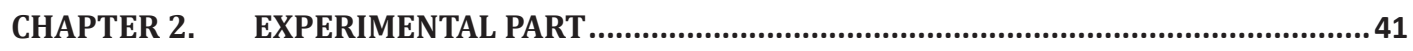

2.1 Preparation of GONRs, PEG-GONRs ANd PEG-MNP-GONRs ............................................. 41

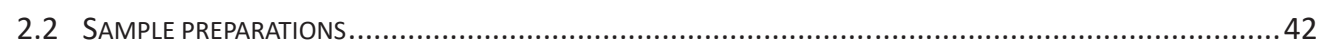

2.2.1 Immobilization of biological and nanomaterials on polyvinylidene difluoride (PVDF)

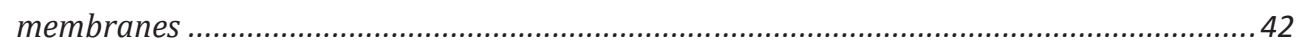

2.2.2 Preparation of tissue, organ and fruit samples................................................... 44

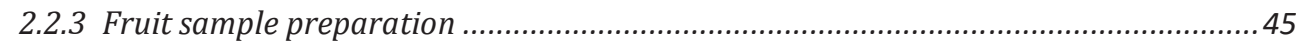

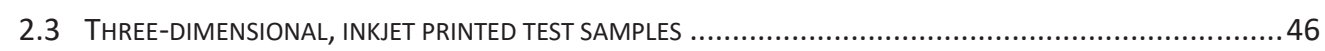

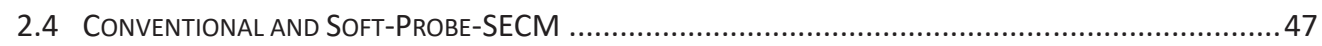

2.4.1 Preparation of conventional Pt electrode ................................................................. 47

2.4.2 Soft stylus probes manufacturing for Soft-Probe-SECM ........................................... 47

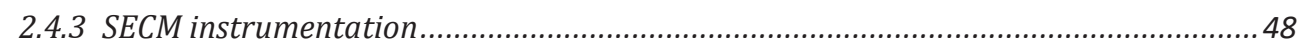

2.5 SECM MEASUREMENTS AND GENERAL MEASUREMENTS PROCEDURES .......................................... 49 
2.5.1 Indirect SECM detection of adsorbed proteins on PVDF membrane by oxygen

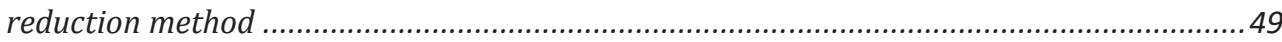

2.5.2 SECM detection of tyrosinase activity immobilized on PVDF membrane .................... 49

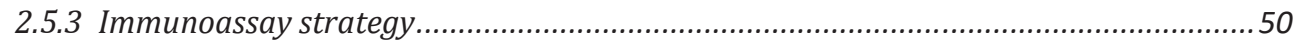

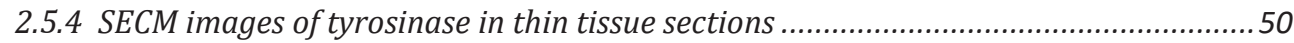

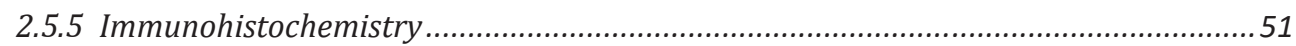

2.6 SINGLE-PHOTON EMISSION COMPUTED TOMOGRAPHY (SPECT)/COMPUTED TOMOGRAPHY (CT)............51

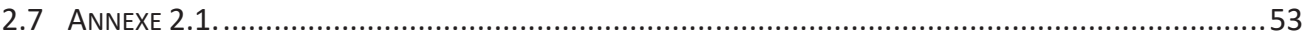

3 MULTIPLE SECM MAPPING OF TYROSINASE IN MICRO-CONTACT PRINTED FRUIT SAMPLES ON POLYVINYLIDENE FLUORIDE MEMBRANE...................................................56

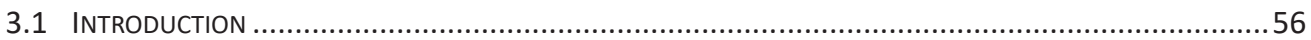

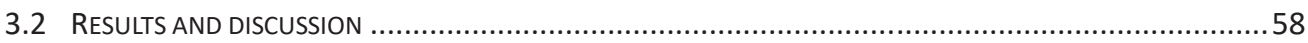

3.2.1 Indirect SECM detection of adsorbed proteins on PVDF membrane by oxygen

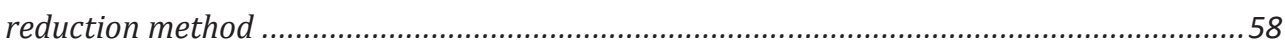

3.2.2 Detection of tyrosinase enzymatic activity by SECM ..............................................62

3.2.3 Mass spectrometry analysis of the enzymatic reaction ...........................................66

3.2.4 SECM immunoassay of tyrosinase immobilized on PVDF membrane ..........................68

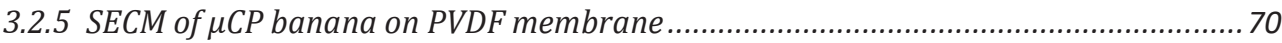

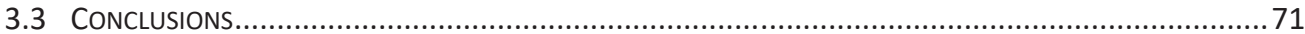

4 MONITORING TYROSINASE EXPRESSION IN NON-METASTATIC AND METASTATIC

MELANOMA TISSUES BY SECM................................................................................................

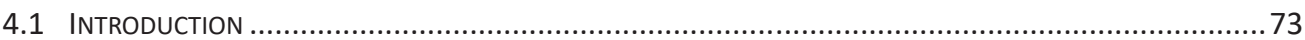

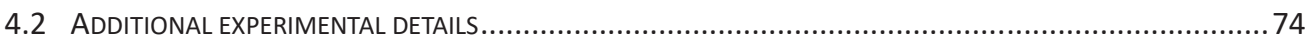

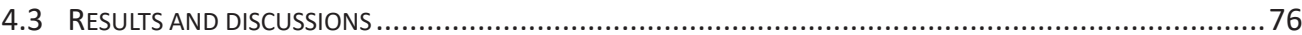

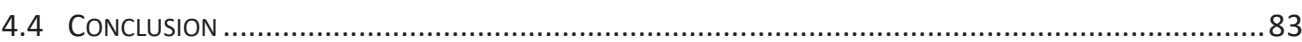

5 FROM TISSUES TO ORGANS: SOFT ELECTROCHEMICAL PROBES FOR SCANNING

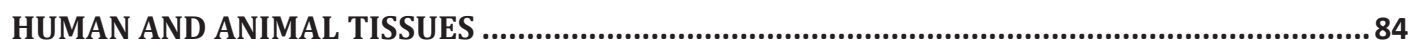

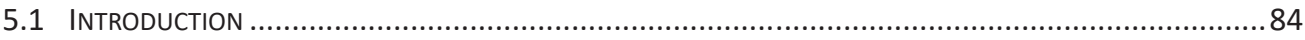

5.2 AdDITIONAL EXPERIMENTAL DETAILS OF SOFT PROBE FABRICATION ............................................. 87

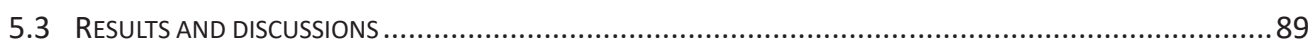

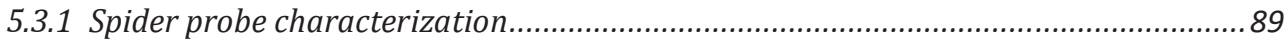

5.3.2 Soft single probe on thick melanoma tissues ........................................................ 97

5.3.3 Thick melanoma tissue scanning by spider probe ................................................. 101

5.3.4 Scanning an entire mouse heart section ............................................................... 103

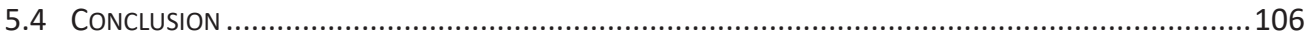

5.5 ADDITIONAL INFORMATION FOR NORMALIZATION OF THE SECM IMAGES....................................... 107 
6 IMAGING THE DISTRIBUTION OF GRAPHENE OXIDE NANORIBBONS IN MICE LIVERS

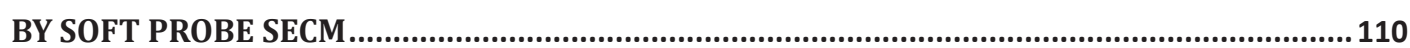

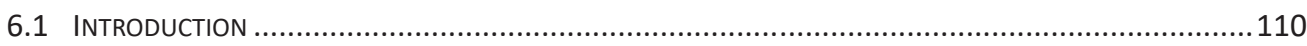

6.2 RESULTS AND DISCUSSIONS ......................................................................................112

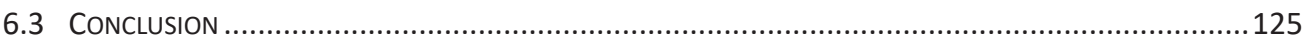

7 MAPPING THE ANTIOXIDANT ACTIVITY OF APPLE PEELS WITH SOFT PROBE

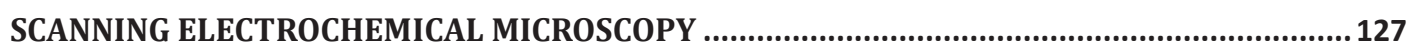

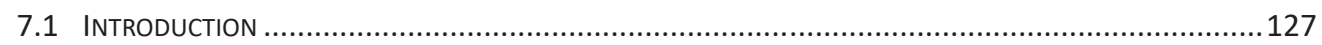

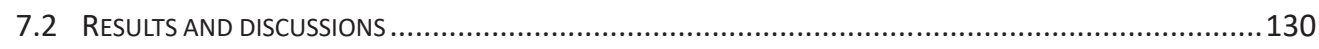

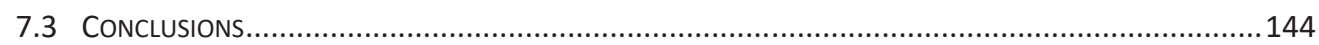

8 GENERAL CONCLUSIONS AND FUTURE PERSPECTIVES................................................ 146

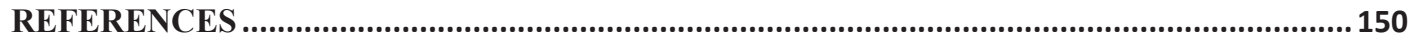




\section{Chapter 1. Introduction}

\subsection{Theory and principles of scanning electrochemical}

\section{microscopy (SECM)}

\subsubsection{The origin of SECM}

SECM is a scanning probe technique that is composed of a micro- or nanoelectrode (ME or NE) that can be positioned or scanned in close proximity to a solid-liquid, air-liquid, or liquid-liquid interface. ${ }^{[1-5]}$ Faradaic current signals can be recorded at the sample and the electrode. Scanning a surface creates usually highly resolved maps of local surface reactivity or mass transport phenomena. The first experiment that is generally considered as an SECM measurement was reported in 1986 by Engstrom et al. who used a microelectrode as sensor for monitoring the concentration profile of a redox species inside the diffusion layer of a macroscopic electrode ${ }^{[6]}$ A contemporary progress performed by Bard and co-workers showed large currents in electrochemical scanning tunneling microscopy (ECSTM) experiments, when the tip was situated outside the tunneling distance. ${ }^{[7]}$ Quickly, they realized that the nature of such current was not related to tunneling currents, but due to an enhanced flux of redox active species. This inspired Bard and co-workers to formulate a quantitative description of the diffusion-limited current at a ME as a function of the working distance $d$ above a macroscopic planar sample and coined the term "scanning electrochemical microscopy (SECM)". ${ }^{[8]}$

Over the years, SECM has been employed for various applications, for instance to map the topography and surface reactivity of various materials, probe enzymatic activities in living cells, screen electrocatalytic materials for energy research, track oxygen consumption in microtissues and in embryos, and investigate kinetics at the liquid/liquid interface. ${ }^{[9-20]}$ In order to satisfy various types of applications and experiments, several methods for the fabrication of different types of SECM probes have been developed. For instance, submicrometer glass-encapsulated microelectrodes and self-assembled spherical gold microelectrodes. ${ }^{[21]}$ The tip (often abbreviated as "T") 
typically used for SECM experiments is made of a microwire of $\mathrm{Pt}$, or $\mathrm{Au}$ or a carbon embedded in an insulating material like glass and connected to a thicker metallic wire for electrical connection via $\mathrm{Ag}$ epoxy resin or Sn soldering (Fig.1.1a). Finally, the active area of the electrode is exposed by mechanical polishing until a flat, smooth and centered electrode is obtained in the shape of a microdisk (typically with diameters below $25 \mu \mathrm{m}$, Fig.1.1b).
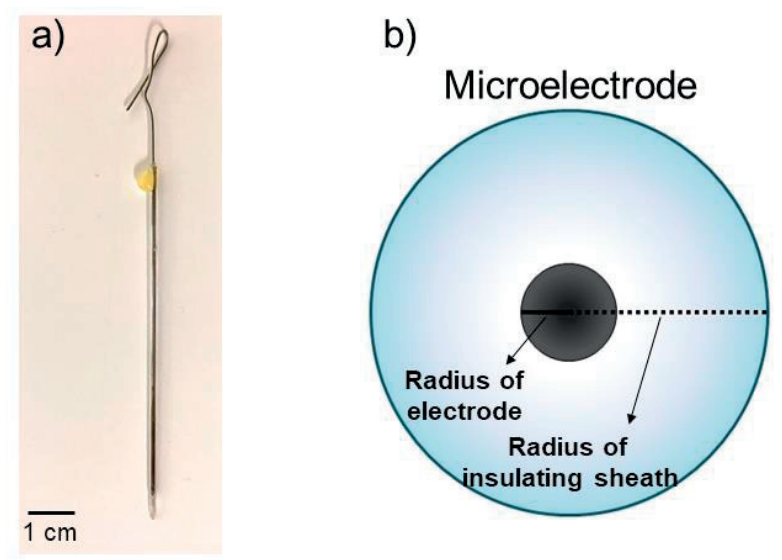

Fig.1.1. a) Optical image of a typical glass-Pt ME. b) The ratio between the radius of the insulating sheath and the radius of the electrode is called $R G$.

Microelectrodes, with the at least one dimension in the micrometer range, ${ }^{[22]}$ possess several special features and advantages for electrochemical experiments in comparison to macroelectrodes, such as low ohmic drop, low capacitive current and fast mass transport. ${ }^{[23-25]}$ For instance, with macroelectrodes the mass transport profile is mainly perpendicular to the electrode surface and a one-dimensional linear diffusion field continuously grows, which results in a time dependence of the measured current under mass-transport control conditions (Fig.1.2). However, as the size of the electrode becomes smaller, e.g. in the low micrometer range, the contribution of species diffusing from the electrode edge becomes more important and as a consequence the diffusion layer grows not only perpendicular, but also parallel to the surface of the electrode leading to the development of a hemispherical diffusion profile with finite size (Fig.1.2b). Thus, the amount of species reaching the surface of the electrode per unit of area and time is much higher than in the case of a macroelectrode. 
a) Linear diffusion at a macroelectrode

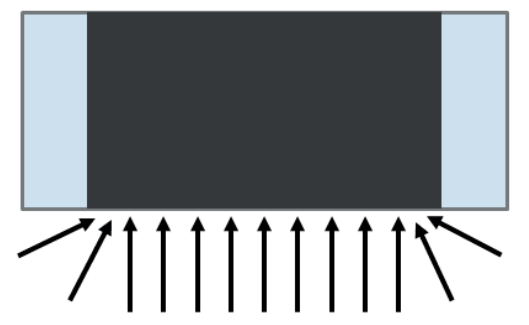

b) Hemispherical diffusion at a microelectrode

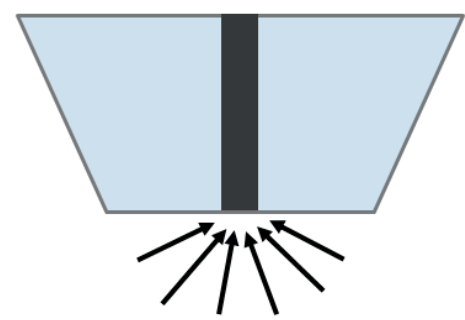

Fig.1.2 Schematic representation of a) linear diffusion at a macroelectrode and b) hemispherical diffusion at a microelectrode.

Indeed, such situation produces a rapid equilibrium on the flux of species in and out of the diffusion layer volume generating a time independent diffusion layer. ${ }^{[26]}$ This contributes to the rapid establishment of a steady-state diffusion-limited current $i_{T, \infty}$ that for a disk-shaped electrode is defined by: ${ }^{[27]}$

$$
i_{\mathrm{T}, \infty}=4 n F D c_{R}{ }^{*} r_{\mathrm{T}} \quad \text { (Equation 1.1) }
$$

where $F$ is the Faraday constant, $n$ is the number of electrons transferred per molecule or ion, $D$ is the diffusion coefficient, $c_{R}{ }^{*}$ is the concentration of a species $\mathrm{R}$ in the bulk solution and $r_{\mathrm{T}}$ is the radius of the disk-shaped microelectrode. This equation can be used for evaluating the size and shape of a microelectrode (if all other parameters except $r_{\mathrm{T}}$ are known), or to determine the diffusion coefficient of a given redox species (if all parameters except $D$ are known). However, this equation is based on the assumption of an infinitely wide insulating plane around the disk electrode suppressing efficiently the diffusion from the back of the microelectrode. The ratio between the radius of the insulating sheath $\left(r_{\text {glass }}\right)$ to the radius of the active electrode area $\left(r_{T}\right)$ has been defined as $R G$ and is an important parameter employed for characterizing the microelectrode (Fig.1.1b). Microelectrodes with a thickness of the insulating sheath similar to the microelectrode radius would present a deviation from this equation due to the diffusion of redox species from the back plane of the electrode towards the surface of the electrode. In such cases, a steady-state diffusion-limited current $i_{\mathrm{T}, \infty}$ for a disk-shaped electrode is defined as:

$$
i_{\mathrm{T}, \infty}=g n F D c_{R}{ }^{*} r_{\mathrm{T}}
$$

(Equation 1.2)

Where $g$ (4 for disk electrode with infinity large insulator) is a factor that represents the 
influence of the insulating geometry on the $i_{\mathrm{T}, \infty}$ recorded at inlaid disk microelectrodes.

Capacitive currents are caused by double layer charging at the interface between the working electrode surface and the solution in which the microelectrode is immersed in. An electrode with a smaller surface area can hold less charge when it is biased and thus reduces the capacitive current. As a result, fast cyclic votammetry (i.e. $1 \mathrm{kV} / \mathrm{s}$ ) can be performed with low impact of capacitive currents, for instance for the detection of reaction intermediaries of very short lifetimes. ${ }^{[28-30]}$ In general terms, the smaller the characteristic dimension of the electrode, the smaller the effect of the capacitive current.

Another advantage of using microelectrodes is the low ohmic $(i R)$ drop that is typically achieved at such electrodes. The $i R$ drop stands for the product of the current (i) and the resistance $(R)$ of the electrolyte solution. Thus, as the current measured with microelectrodes is within the nano- or pico-ampere range, the $i R$ drop tends to be negligible when working with microelectrodes even in highly resistive media. ${ }^{[24,25]}$ Fig.1.3 shows a typical sigmoidal shaped cyclic voltammogram (CV) recorded at a microelectrode with a fairly slow scan rate in an electrolyte solution containing $2 \mathrm{mM}$ $\mathrm{FcMeOH}$ as a redox active species and a supporting electrolyte. In Fig.1.3 the current increases as the applied potential increases. When the applied potential at the microelectrode is high enough, the amount of $\mathrm{FcMeOH}$ molecule being oxidized at the electrode surface reaches the maximum. Therefore, the current is constant and is called a plateau current. 


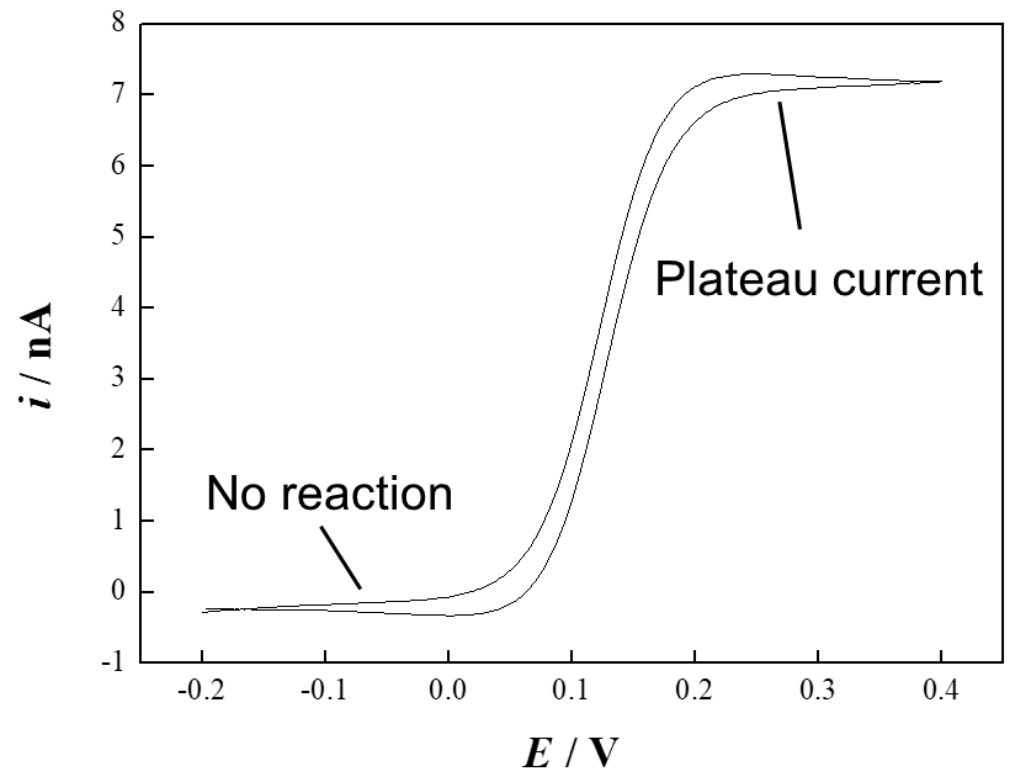

Fig.1.3 Cyclic voltammogram of $2 \mathrm{mM} F \mathrm{FMeOH}$ at the conventional Pt ME. $R G=10, \mathrm{Ag}$ QRE, Pt CE, FcMeOH $2 \mathrm{mM}$ in phosphate buffer, $\mathrm{pH} 6$, scan rate $=50 \mathrm{mV} / \mathrm{s}$.

In addition to measuring the current or potential, SECM has been applied as a tool for creating micro- or nanostructures on a broad range of surfaces. ${ }^{[31-36]}$ SECM can drive chemical reactions such as metal deposition by generating through anodic dissolution metal cations from a sacrificial SECM tip being electrochemically reduced on a biased conducting surface or generating etchants, such as bromine, at the SECM tip to etch and hence microstructure semiconductor surfaces or self-assembled monolayers on $\mathrm{Au} .{ }^{[37]}$ The direct mode and feedback mode are two operation modes commonly used for the surface patterning. The former consists of only two electrodes where the tip is biased $v s$ the substrate. ${ }^{[34,38]}$ Recently, this mode has been used for patterning carboxylation of multilayered graphene on nickel. ${ }^{[38]}$ Carbon dioxide serves as the carboxylation agent and the degree of modification or the surface coverage can be easily controlled by the experimental parameters and the size of SECM tip.

The versatility of the SECM feedback mode made it popular for the fabrication of microstructures. ${ }^{[31]}$ Under the operation of feedback mode, the tip placed close to the substrate can reduce or oxidized the redox active species that can drive microelectrochemical processes on the sample surface. For instance, it can be used to corrode magnesium alloys in $\mathrm{NaCl}$ solutions and control the electrodeposition of metal nanoparticles on single walled carbon nanotubes. ${ }^{[39,40]}$ Moreover, the microstructuring procedures can be fastened by using soft linear arrays of carbon microelectrodes. Lesch 
et. al. used a soft linear microelectrode array for drawing and reading modified selfassembled monolayers on an Au surface. ${ }^{[41]}$

\subsubsection{Instrumentation of SECM}

The basic SECM set up consists of hardware components to precisely position and scan a microelectrode as a local probe near a sample surface (Fig.1.4). For the purpose of controlling the position of the probe, the microelectrode is attached to a fine $x, y, z$ positioning system composed of piezoelectric, stepper or hybrid motors. Additionally, a (bi)potentiostat is required to control the applied potential at the tip and/or the substrate, and to measure the current produced at the substrate and/or the tip. Since low current values (i.e. $\mathrm{pA}$ to $\mathrm{nA}$ ) are measured and high precision probe positioning (i.e. $\mathrm{nm}$ range) is required, electromagnetic noise and mechanical vibrations should be avoided by isolating the SECM setup inside a Faraday cage and by placing it on top of an anti-vibration table. However, rapid movements of the tip can cause convection in the solution around the microelectrode, which perturbs the diffusion of redox active species and thus have influences on the measurement results, especially for high-resolution experiments. As a result, experiments with translation speeds higher than $10 \mu \mathrm{m} / \mathrm{s}$ could already show some influence on the obtained results. ${ }^{[42]}$ A delay time (i.e. the time between probe translation and data acquisition; in this work simply abbreviated as ADC for analog-to-digital conversion) can be applied to avoid the interference from convection. This results in a stop and go movement of the probe. Some commercial SECM systems, such as the one from CHI instruments measure continuously while the probe is moving and SECM images distorted by convection can be found in literature. SECM imaging is a line-by-line experiment and long experimental times can result when large areas $\left(e . g .1 \mathrm{~cm}^{2}\right)$ are scanned. In such cases, a compromise between SECM imaging resolution and probe translation rate (hence imaging time) is generally found. Various approaches have been developed to decrease the imaging time and are discussed in Chapter 5. 


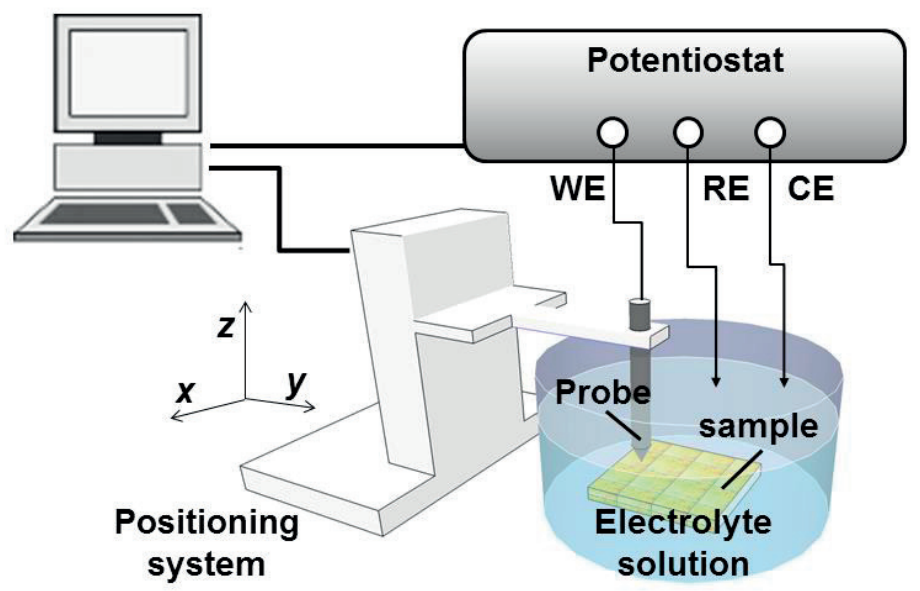

Fig.1.4 Basic setup of SECM consisting of a microelectrode probe, a potentiostat, that controls a three electrode cell (i.e. working electrode (WE), reference electrode (RE) and counter electrode (CE)). The positioning system allows the probe moving in $x, y, z$ directions.

\subsubsection{Main operation modes of SECM}

Different operation modes of SECM have been reported, such as the feedback mode, tip generation/substrate collection (TG/SC) mode as well as substrate generation/tip collection (SG/TC) mode, penetration mode and redox competition mode. ${ }^{[37,43-45]}$ However, the feedback mode and the generation/collection mode are the most widely used for SECM imaging.

\section{SECM Feedback Mode}

Under feedback mode operation, the tip is immersed in the bulk of a solution containing an electroactive species, e.g. the reduced species $\mathrm{R}$. The microelectrode is biased to a potential so that the redox mediator can be oxidized to $\mathrm{O}$. At a large distance between the tip and the substrate, a steady-state diffusion-limited current $i_{\mathrm{T}, \infty}$ is established due to the hemispherical diffusion field around the microelectrode (Fig.1.5a). When the biased microelectrode is approached within a few tip radii towards a flat electrical insulator or non-active substrate, the recorded current at the tip $\left(i_{T}\right)$ decreases as the tip gets closer to the substrate. This behavior is called negative feedback, which is a consequence of the physical hindering of the diffusion of the redox mediator to the electrode surface by the substrate (Fig.1.5b) producing a decrease of the recorded current $\left(i_{T}<i_{T, \infty}\right)$. 
Conversely, if the probe is approaching a conductive or chemically active substrate, the oxidized form of the redox active species $(\mathrm{O})$ previously formed at the microelectrode can immediately be reduced back to $\mathrm{R}$ at the sample surface (Fig.1.5c). This recycling process leads to an increase of the recorded current at the tip $\left(i_{T}\right)$, which can become larger than the current in the bulk solution $\left(i_{T}>i_{T, \infty}\right)$. The parameters that affect the signal measured include concentration of redox mediator, tip-substrate distance, the size of the sample area and the electron transfer rate. Therefore, it is possible to quantify the conductivity of thin films by SECM through adjusting these variables.

$$
\mathrm{R} \rightarrow \mathrm{O}+n e^{-}
$$

a)

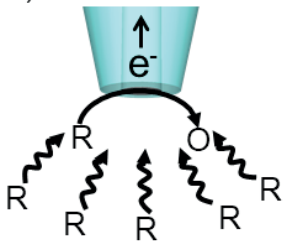

b)

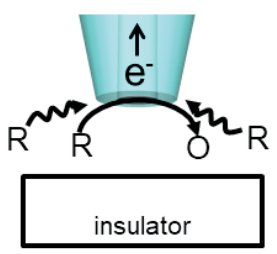

(Equation 1.3)

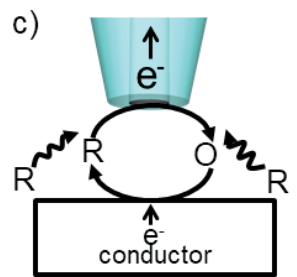

Fig.1.5 Schematic representation of SECM feedback operation mode. a) Hemispherical diffusion field around the microelectrode, $\mathrm{R}$ stands for the reduced form of a redox couple. The microelectrode is in the bulk solution and far away from the substrate. $b$ ) Diffusion of $R$ towards the microelectrode is hindered by the substrate surface in the negative feedback mode. c) Species $\mathrm{R}$ is regenerated at the substrate in the positive feedback mode.

If the probe is approached toward an unbiased conductive substrate immersed in a solution of redox-active species $\mathrm{R}$, the substrate potential $\left(E_{\mathrm{S}}\right)$ is given by the concentration ratio between $\mathrm{R} / \mathrm{O}$ at the vicinity of the interface between the solution and substrate below the microelectrode. Indeed, due to the local perturbation introduced by the microelectrode at the vicinity of the conductive substrate, different potential regions can be generated. The oxidation of $\mathrm{R}$ and the reduction of $\mathrm{O}$ on the same surface of the conductive substrate can take place owing to the lateral charge transport inside the conductive film (Fig.1.6). 


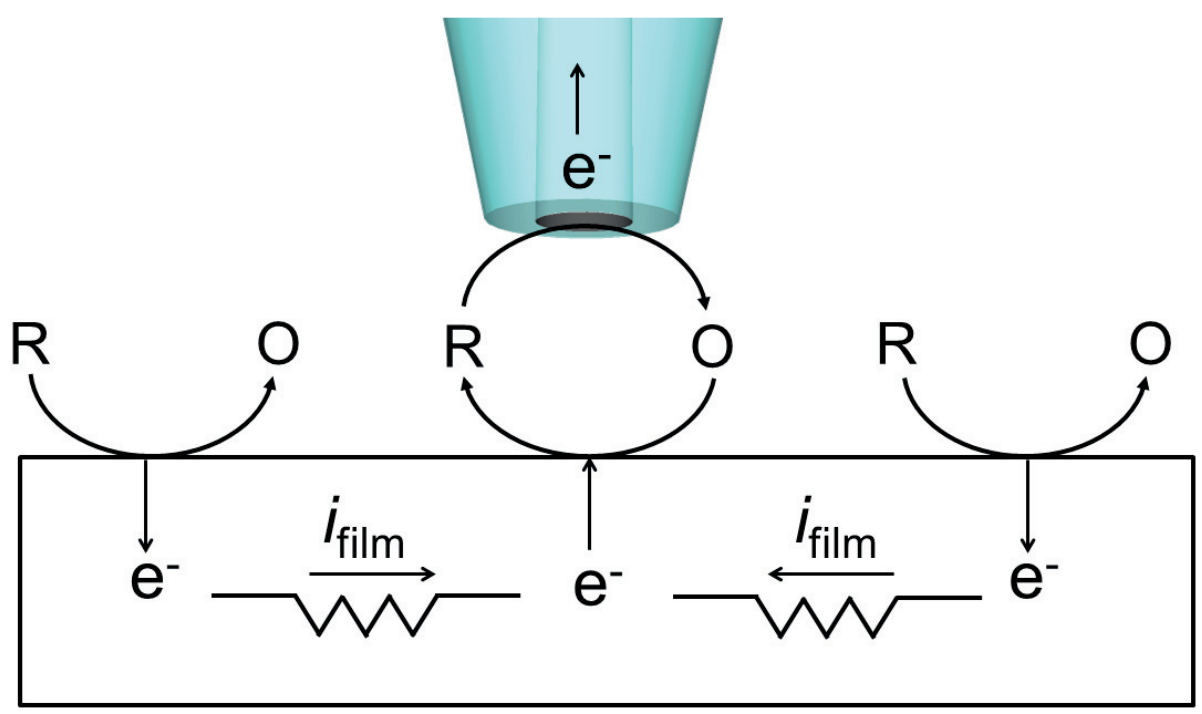

Fig.1.6 Schematic diagram showing the recycling of the redox mediator and the charge transfer processes across an unbiased conductive substrate. ${ }^{[46]}$

Fig.1.7 shows an example of negative feedback and positive feedback approach curves, which correspond to the relationship of $i_{T}$ versus $d$. To consider the measurements using different redox mediators or electrodes with slightly different electroactive area, normalizations of the currents and distance are usually performed. $L$ is the normalized distance defined by the ratio of the tip-substrate separation $d$ to the radius of the electrode $r_{\mathrm{T}} . I$ is the normalized current defined by the ratio of the tip current $i_{\mathrm{T}}$ recorded at any $z$ position to the current in the bulk of the solution $i_{\mathrm{T}, \infty}$. 


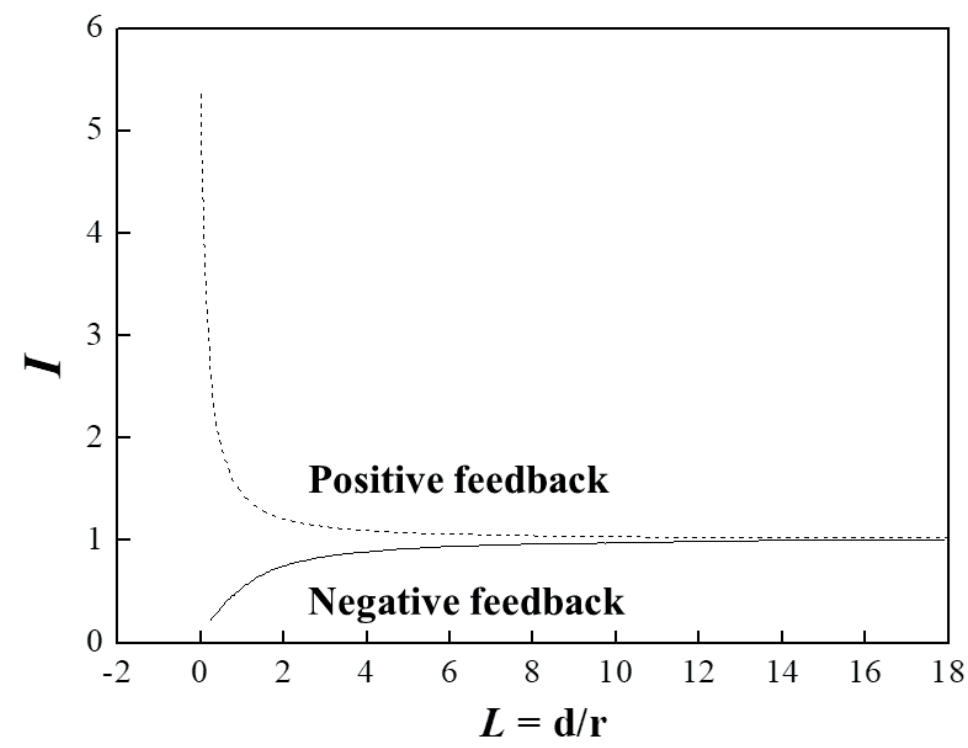

Fig.1.7 Normalized SECM approach curves of negative and positive feedback. Where $L$ is the normalized distance defined by the ratio of the tip-substrate separation $d$ to the radius of the electrode $r_{\mathrm{T}} . I$ is the normalized current defined by the ratio of the tip current $i_{\mathrm{T}}$ recorded at any $z$ position to the current in the bulk of the solution $i_{\mathrm{T}, \infty}$.

SECM approach curves are very useful since they do not only provide information about the position of the sample surface in regard to the SECM tip, but also about the kinetics of the chemical reactions taking place at the substrate. Cornut et al. proposed recently an approximated analytical expression for SECM approach curves, where the $R G$ of the probe and the finite kinetics of the process are taken into account: ${ }^{[47]}$ $I_{\mathrm{T}}(L, \Lambda, R G)=I_{\mathrm{T}}^{\text {active }}\left(L+\frac{1}{\Lambda}, R G\right)+\frac{I_{\mathrm{T}}^{\text {inactive }}(L, R G)-1}{\left(1+2.47 R G^{0.31} L \Lambda\right)\left(1+L^{0.006 R G+0.113} \Lambda^{-0.0236 R G+0.91}\right)}$ (Equation 1.4)

Where the normalized currents for active $\left(I_{\mathrm{T}}^{\text {active }}\right)$ and inactive $\left(I_{\mathrm{T}}^{\text {inactive }}\right)$ substrates are equal to 


$$
\begin{gathered}
I_{\mathrm{T}}^{\text {active }}\left(L+\frac{1}{\Lambda}, R G\right)=\alpha(R G)+\frac{\pi}{4 \beta(R G) \operatorname{ArcTan}\left(L+\frac{1}{\Lambda}\right)} \quad \text { (Equatio } \\
+\left(1-\alpha(R G)-\frac{1}{2 \beta(R G)}\right) \frac{2}{\pi} \operatorname{ArcTan}\left(L+\frac{1}{\Lambda}\right) \\
I_{\mathrm{T}}^{\text {inactive }}(L, R G)=\frac{\frac{2.08}{R G^{0.358}}\left(L-\frac{0.145}{R G}\right)+1.585}{\frac{2.08}{R G^{0.358}}(L+0.0023 R G)+1.57+\frac{\ln R G}{L}+\frac{2}{\pi R G} \ln \left(1+\frac{\pi R G}{2 L}\right)}
\end{gathered}
$$

(Equation 1.6)

And with

$$
\alpha(R G)=\ln 2+\ln 2\left(1-\frac{2}{\pi} \operatorname{ArcCos}\left(\frac{1}{R G}\right)\right)-\ln 2\left(1-\left(\frac{2}{\pi} \operatorname{ArcCos}\left(\frac{1}{R G}\right)\right)^{2}\right)
$$

(Equation 1.7)

$$
\beta(R G)=1+0.639\left(1-\frac{2}{\pi} \operatorname{ArcCos}\left(\frac{1}{R G}\right)\right)-0.186\left(1-\left(\frac{2}{\pi} \operatorname{ArcCos}\left(\frac{1}{R G}\right)\right)^{2}\right)
$$

(Equation 1.8)

$$
\Lambda=\frac{k r_{T}}{D} \quad \text { (Equation 1.9) }
$$

Where $\Lambda$ is the kinetic constant, $k$ is the first order heterogeneous rate constant and $D$ is the diffusion coefficient of the redox species. Thus, by fitting the theoretical approach curve to the experimental ones, $k$ can be deduced from $\Lambda$ as obtained from fitting procedure. All other parameters can be determined independently or can be found in the literature (e.g., D). Fig.1.8 shows several calculated approach curves by using different $k$ values. If $k$ values are higher or equal to $0.1 \mathrm{~cm} \mathrm{~s}^{-1}$, the approach curves present a fast increase when the working distance decreases and basically overlap with the theoretical fully positive feedback approach curve. However, when $k$ takes smaller values, the approach curves deviate clearly from the positive feedback behavior and after passing by an intermedium regime, it reaches the theoretical fully negative 
feedback behavior and $k$ is around $0 \mathrm{~cm}^{-1}$.

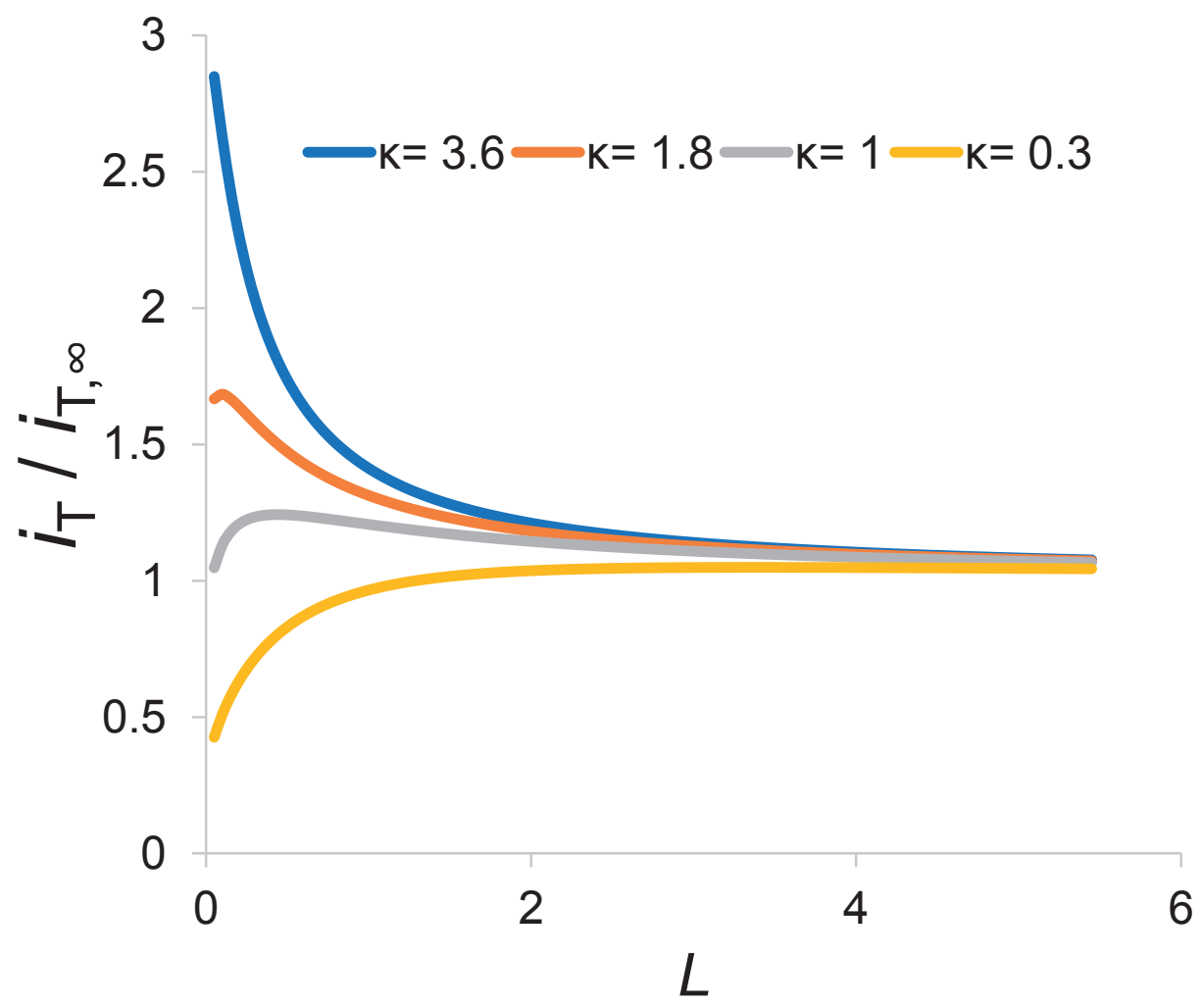

Fig.1.8 Influence of the electrochemical reaction rate that takes place at the substrate on the SECM approach curves. ${ }^{[47]}$

By using the previous model (equation 3), it can also be depicted the influence that the $R G$ has over the approach curves (Fig.1.9). For instance, probes with larger $R G$ values provide a higher current contrast in SECM feedback mode, as at close working distances $(L<2)$ the separation between the positive and negative approach curves is higher than in the cases of smaller $R G$ values. The latter is mainly due to the most efficient hindering of the diffusion of the redox mediator towards the surface of the electrode with a bigger insulating sheath. In spite of the better current contrast provided by larger $R G$ value probes, experimentally it is more difficult to bring the probe close to the substrate (without any probe-substrate crash) due to the potential misalignment between the plane of the probe and the substrate, which originates from the difficulty of placing the microelectrode and sample perfectly vertically or horizontally, respectively. Therefore, depending on the goal of the experiment, a compromise has to be made to achieve a good current contrast, while avoiding probe-substrate crashes. 


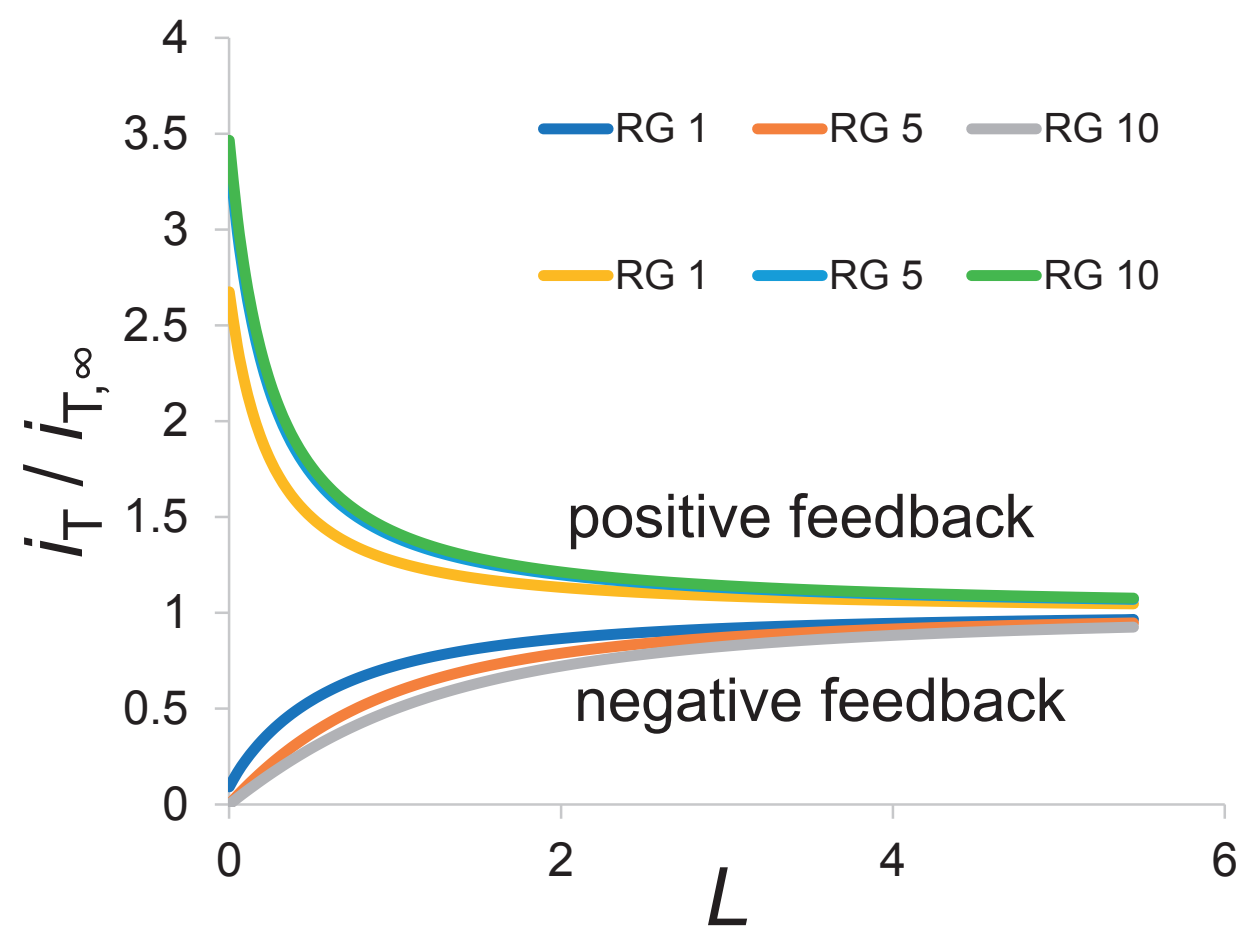

Fig.1.9 Effect of $R G$ on SECM approach curves for positive and negative feedback. $R G=1$, 5 , and 10 .

\section{Substrate Generation/Tip Collection Mode}

As a matter of fact, the SECM substrate generation/tip collection (SG/TC)) mode (Fig.1.10) was carried out in the first SECM-like experiment for measuring the concentration profile of a redox species inside the diffusion layer generated at a macroscopic electrode. ${ }^{[6]}$ In the SG/TC mode, the substrate can convert chemically or electrochemically species $\mathrm{R}$ into species $\mathrm{O}$ that diffuses away from the substrate and can be collected at the SECM probe. Consequently, $i_{\mathrm{T}}$ reflects the concentration profile of the redox species around an active site of the sample. Similarly, as the feedback mode, qualitative and quantitative information can be extracted by correlating the mass transport regime with the kinetics of the process. In general, this mode is often used for monitoring enzymatic reactions, corrosion and electron transfer processes at different interfaces. ${ }^{[48-50]}$ For instance, Bard's group monitored spatially the activity of immobilized glucose oxidase based on the detection of hydrogen peroxide at an SECM tip. ${ }^{[51]}$ The method provided an excellent spatial sensitivity for hydrogen peroxide and thus allowing the monitoring of the catalytic activity of immobilized enzymes. The 
main advantages of using the SG/TC or tip generation/substrate collection (TG/SC) mode are less background currents and the less dependence of the working distance on the probe response. This enables the measurements at larger distances between the probe and the substrate that could be beneficial when studying samples with special topography. Furthermore, SG/TC has been widely applied to the study of biological samples, for instance when the metabolic response from a cell is expressed as a chemical species released to the extracellular medium and detected at the scanning probe. ${ }^{[52]}$ All in all, the lateral resolution could decrease compared to feedback mode SECM. To overcome these drawbacks, smaller probes and slower scan rates and smaller step sizes are preferred.

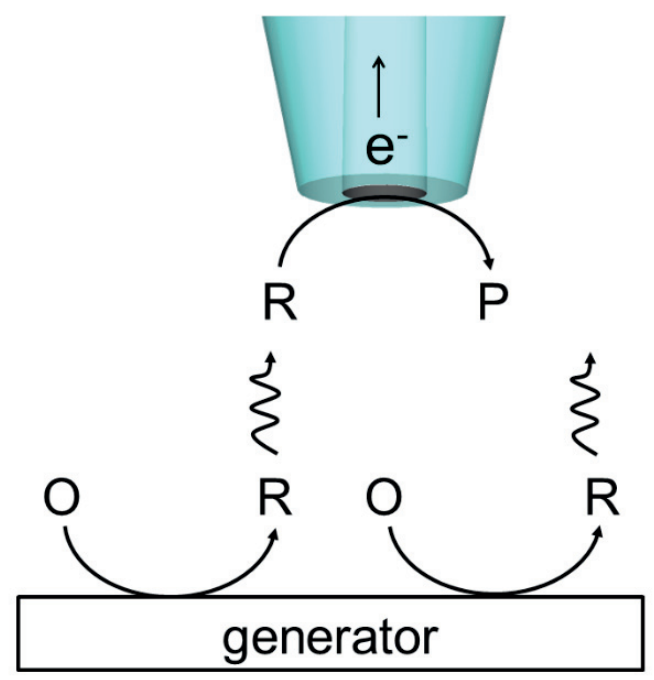

Fig.1.10 Schematic diagram of substrate generation/tip collection mode. Electrochemically active species $\mathrm{O}$ is generated at the substrate surface and collected at the tip. ${ }^{[43]}$ 


\subsubsection{SECM applied to biological samples}

SECM can be used to image the topography and reactivity of biological specimens, as well as, to detect electrochemically metabolites for mapping localized biochemical activity. ${ }^{[53-57]}$ Among the SECM operation modes, generation/collection and feedback mode measurements are the two main SECM modes employed to study biological samples. For instance, Takahashi's group imaged an important membrane protein, i.e., epidermal growth factor receptor (EGFR), on the surface of a single living mammalian cell by SECM SG/TC mode. ${ }^{[58]}$ The EGFR is one of the key membrane proteins associated with cancer. In order to estimate EGFR expression levels by SECM, EGFR on the cell surface was labeled with alkaline phosphatase (ALP) via an antibody. Thus, in presence of $\mathrm{p}$-aminophenol phosphate (PAPP, the enzymatic substrate of ALP) ALP catalyzes enzymatically the production of p-aminophenol (PAP), which can be detected electrochemically and hence used to monitor spatially the presence of EGFR. The main advantage of such method provided the information about EGFR level in situ without peeling the living cell.

In feedback mode experiments, the tip is used to oxidize or reduce a redox mediator, and hence the topography and redox activity of biological samples can be examined according to the feedback currents. Mirkin's group used SECM feedback mode to demonstrate the possibility of distinguishing between non-transformed human breast epithelial cells (MCF-10A) and metastatic breast cells (MDA-MB-231) based on their redox activities. ${ }^{[59]}$ With this aim, a chemical mediator (e.g. quinone) was employed that rapidly crosses the cell membrane, participates in intracellular redox reactions and then goes out of the cell to be detected at the microelectrode positioned close to the cell membrane.

Although SECM has been applied to different biological systems, SECM studies of tissues are still under exploration. In most of the cases, the samples which were scanned are "micro tissues" instead of real tissues. For instance, Sridhar et al. monitored the respiratory activity of $3 \mathrm{D}$ cellular aggregates or "micro tissues" by SECM. ${ }^{[18]}$ In their approach, a microwell array for large-scale production of microtissues was stamped on a Petri dish and in situ SECM analysis was performed on the present micro tissues. For instance, the respiratory activity of live HeLa microtissues was assessed by monitoring the oxygen consumption over the cell aggregates at a constant height mode. Similarly, non-invasive SECM assays for detecting osteogenic differentiation on arrays 
of microtissues have been also reported.$^{[17]}$

Although numerous approaches have been applied to the study of tissues, such as colorimetric and fluorometric assays, ${ }^{[60,61]} \mathrm{SECM}$ can provide a more sensitive spatial detection of biomarkers in comparison to colorimetric methodologies, and a wider range of information can be extracted since not only the fluorescent tagged species can be detected. Moreover, when studying living cells by optical methods, genetic modifications or the addition of fluorescent tags are required affecting not only their energy status, but also their whole metabolic response. On the contrary, SECM can be used to monitor natural cell responses like the production of metabolites or the uptake of drugs. ${ }^{[62,63]}$

\subsubsection{Limitations of SECM for real samples and approaches to overcome them}

However, the study of real samples requires overcoming several drawbacks encountered in SECM, such as the fact that the probe response varies depending not only on the chemical reactivity of the surface, but also on the working distance. As a consequence, it is required to maintain a constant $d$ during scanning to avoid topographic artifacts (e.g., with tilted or rough samples). Furthermore, when scanning large area samples (i.e, $1>\mathrm{cm}^{2}$ ) experimental times could be as long as $24 \mathrm{~h}$. The scan rate cannot be increased infinitely otherwise the effect of convection cannot be ignored. ${ }^{[42]}$ This limitation and approaches to overcome such will be described in Chapter 5. As a result, artifacts generated by solvent evaporation, sample aging or electrode fouling can take place. Finally, probe-substrate crashes, i.e. a physical contact between probe and sample, can lead to the damage of the fragile probe or the sample if the working distance becomes zero due to the sample topography. The latter is critical in the case of biological samples, since the hard glass body of the commonly used microelectrodes can scratch cells easily upon contact. To alleviate the above mentioned SECM limitations, several approaches have been proposed where mainly additional instrumentation or routines are incorporated into the basic SECM setup in order to control, for instance, the working distance during scanning (e.g., SECM-atomic force microscopy (AFM), shear force, alternating current (AC)-SECM, among others). ${ }^{[54,64-}$ ${ }^{69]}$ Alternatively, a new family of SECM probes, so-called soft stylus microelectrodes, has been developed recently to carry out SECM experiments of rough, tilted, and large 
substrates in contact mode without the need of any additional equipment (Fig.1.11). ${ }^{[10,12,41,70-72]}$

a) Scan direction

Contact mode Constant height mode

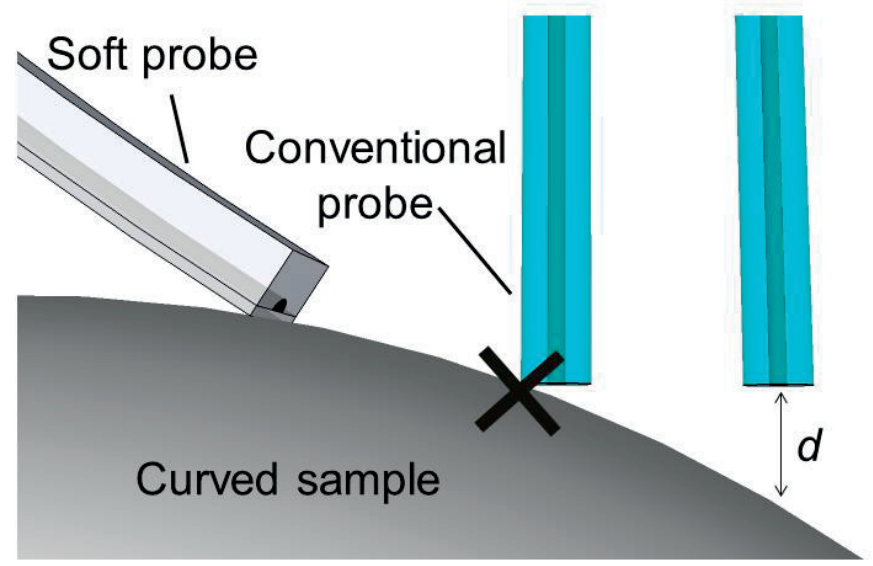

b)

c)
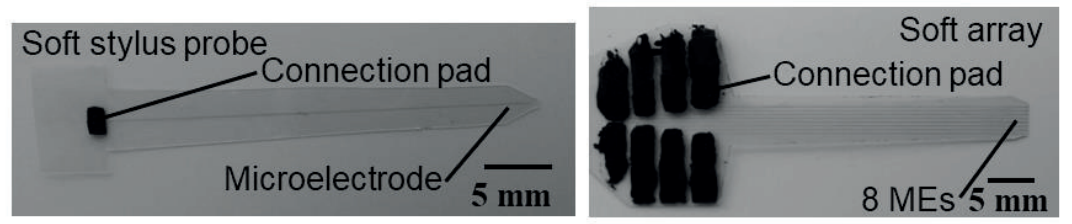

Fig.1.11 a) Schematic representation of lateral SECM scanning over a curved sample with a conventional microelectrode (right) and a soft probe (left). Photographs of a soft stylus probe (b) and a soft array (c).

\subsubsection{Soft-Probe-SECM}

The fabrication of soft stylus probes is based on the UV-photoablation of microchannels into thin polyethylene terephthalate (PET) films (Fig.1.12). Then, a carbon ink is manually applied into the microchannels to create a carbon track that is cured thermally and finally enclosed by a thin layer of Parylene C. The active electrode area is simply exposed by blade or laser-assisted cutting. Soft probes allow SECM imaging in contact mode of strongly tilted and curved substrates, since the flexibility of the soft probes allows the probe body to follow the topography of the sample surface. As a result, an almost constant working distance is achieved avoiding changes in the recorded current related to different topographic artifacts. Furthermore, the soft stylus probe concept can be multiplexed into an array format (Fig.1.11c), so that larger sample areas can be scanned in a shorter time. ${ }^{[41,70,71]}$ Further functionalization of the soft 
probes has led to the integration of microfluidic channels allowing the delivery of a given electrolyte solution at the tip of the probe and the extraction of the species generated from the interaction with the sample. ${ }^{[73,74]}$

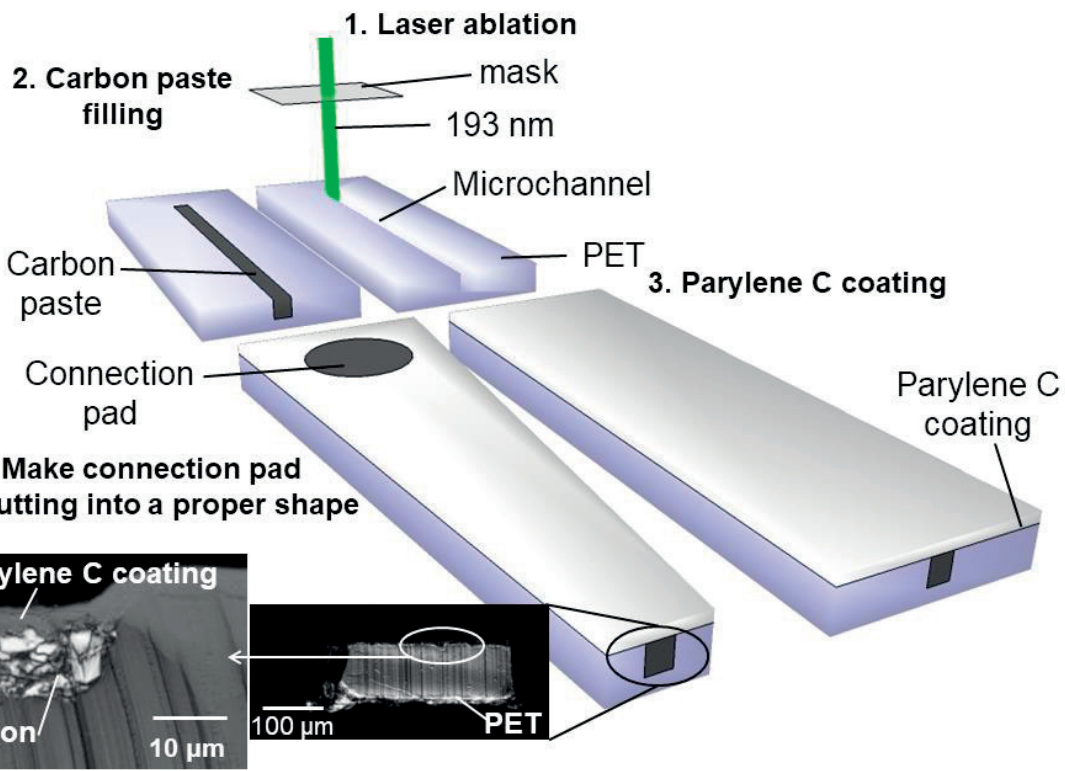

Fig.1.12 a) Soft probe fabrication: 1.) UV laser ablation of a microchannel in a PET sheet, 2.) filling of the microchannel with a carbon paste followed by a curing process at $80^{\circ} \mathrm{C}, 3$.) covering and sealing of the conductive carbon track with a Parylene C coating. 4.) Shaping of the probe body and preparation of the electrical connection pad. The active microelectrode area is exposed by razor blade or laser assisted cutting.

In order to plot the approach curves after touching of the sample, a vertical coordinate $h_{\mathrm{p}}$ is applied (Fig.1.13). It is the difference $h_{\mathrm{P}}=h_{\mathrm{A}}-l_{\mathrm{T}}$ between the height of the attachment point above the sample $h_{\mathrm{A}}$ and the vertical extension $l_{\mathrm{T}}$ of the unbent probe. Thus, the effective working distance $d$ is defined as

$$
\begin{array}{cc}
d=h_{\mathrm{p}}+t_{\mathrm{L}} \sin (\alpha) ;\left(h_{\mathrm{p}} \geqq 0,\right. \text { non-contact region) } & \text { (Equation 1.10) } \\
d=t_{\mathrm{L}} \sin (\alpha) ;\left(h_{\mathrm{p}}<0,\right. \text { contact region) } & \text { (Equation } 1.11)
\end{array}
$$

where $\alpha$ is the angle between the cross-sectional plane of the probe and the sample surface and $t_{\mathrm{L}}$ is the thickness of the Parylene $\mathrm{C}$ film covering the carbon tracks. The geometry of the probes in contact and non-contact regime is shown in Fig.1.13a. 
a) 3. Sliding $\quad$ Just in contact 1 . Non contact

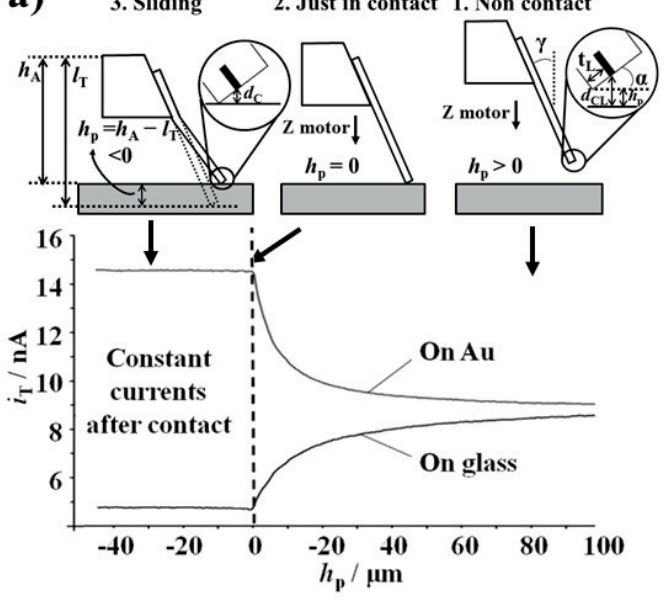

Contact less:

$d_{\mathrm{CL}}=h_{\mathrm{p}}+t_{\mathrm{L}} \cdot \sin (\alpha) ; h_{\mathrm{p}} \geq 0$

Contact:

$d_{\mathrm{C}}=t_{\mathrm{L}} \cdot \sin (\alpha) ; h_{\mathrm{p}}<0$

b)

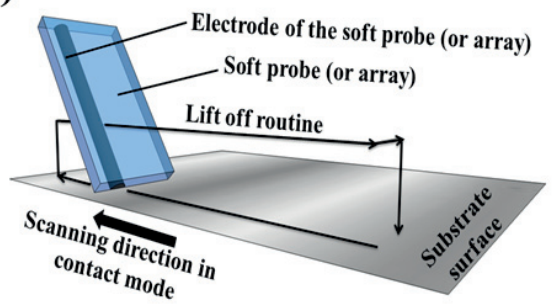

Fig.1.13 a) Recording current-distance curves with a soft probe on gold and glass surfaces. b) Schematic representation of soft probe translations during SECM imaging. The data of approach curves were kindly provided by Dr. Andreas Lesch.

SECM imaging is performed by translating the probe in the horizontal $x$-y-plane while recording a current at defined grid points. The scanning mode using the soft probes is different from typical SECMs where the probe is moved forwardly and reversely in an identical manner. Due to its predefined bending direction, the soft probe needs to be retracted using a lift off mode after a contact mode line scan and while repositioning the probe with a small displacement perpendicularly to the scanning direction (Fig.1.13b). In this way, over-bending and mechanical stress to the probe as well as to the substrate do not occur. In order to avoid double scanning of surface areas with the adjacent microelectrodes of a soft array (the typical step size is 5-10 $\mu \mathrm{m}$, microelectrode separation $500 \mu \mathrm{m}$ ), a larger displacement is performed to image a new frame next to the previously measured region.

\subsection{Melanoma}

\subsubsection{Melanoma classification}

Melanoma is one of the most lethal cutaneous malignancies that strike thousands of people around the world. According to the US Cancer Statistics report, there were 76,100 new cases of melanoma incidence in the U.S. in 2014. ${ }^{[75]}$ The causes of melanoma can be abnormal immunosuppression or ultraviolet radiation that induces 
genetic mutation in the skin. The stepwise progression of melanocytes to malignant melanoma is depicted by the Clark model in Fig.1.14. ${ }^{[76]}$ The characters of five levels of melanoma are $i$ ) the proliferation of the melanocytes in the process of forming nevi, which is also called melanoma in situ. ii) The increase of aberrant cell growth and subsequent development of dysplasia and the invasion into the papillary dermis (below the epidermis). iii) The radial-growth phase (RGP) leads to the invasion to the junction of the papillary and dermis. $i v$ ) The lesion invades dermis and progresses to vertical growth phase (VGP). v) The metastasis of melanoma to other organs. Clinically, the staging criteria of melanoma is suggested by American Joint Commission on Cancer (AJCC) which classifies melanoma according to the tumor, node, metastasis system (TNM) (Table 1.1). ${ }^{[77,78]}$ The survival rate of patients depends on the stage of the cancer when it is diagnosed. Therefore, reliable methodologies for early diagnosis and unequivocal identification of cancer stages are of high relevance. ${ }^{[79,80]}$ The appearance of melanoma and innocuous skin moles are sometimes similar. Thus, the early detection of melanoma is still a challenge.

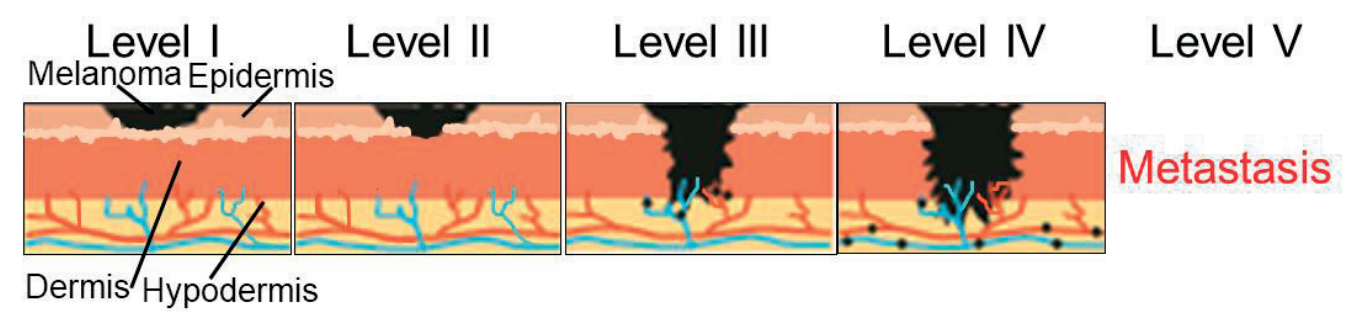

Fig.1.14 Melanoma progression according to Clark model.

Table 1.1 Staging system of melanoma suggested by AJCC. ${ }^{[77,78]}$

\begin{tabular}{l|l} 
Stage & Criteria \\
\hline $\boldsymbol{I A}$ & Non-invasive localized melanoma with thickness smaller than $0.75 \mathrm{~mm}$. \\
$\boldsymbol{I B}$ & Non-invasive localized melanoma with thickness of 0.76-1.5 mm. \\
$\boldsymbol{I I A}$ & Non-metastatic localized melanoma with thickness of 1.5-4 mm. \\
$\boldsymbol{I I B}$ & Non-metastatic localized melanoma with thickness larger than $4 \mathrm{~mm}$. \\
$\boldsymbol{I I I}$ & Invasion of regional lymph node and limited metastasis but without nodal \\
& metastases \\
$\boldsymbol{I V}$ & Advanced regional metastases or distant metastases
\end{tabular}




\subsubsection{Tyrosinase and its enzymatic reactions}

Tyrosinase (EC 1.14.18.1) is a multifunctional copper-containing enzyme from the oxidase superfamily. Tyrosinase is also the key enzyme involved in the biosynthesis of melanin, which is the entity in charge to protect the skin from UV radiation. This enzyme catalyzes the first two steps of melanin synthesis that corresponds to the hydroxylation of monophenol derivative to $o$-diphenol (monophenolase or cresolase activity) and the oxidation of $o$-diphenol to $o$-quinone (diphenolase or catecholase activity, see Fig.1.15). ${ }^{[81,82]}$ The further steps of melanin formation involve a series of non-enzymatic steps, which are typically faster than the enzymatic ones. ${ }^{[83,84]}$ Normally, melanin is synthesized in epidermal melanocytes and then it is transferred into epidermal keratinocytes through the melanocytes' dendrites. ${ }^{[85]}$ Subsequently, melanin is distributed by melanocytes within the skin or the hair. Melanin formation is of high importance, and abnormalities on its synthesis can lead to problems related to skin photocarcinogenesis $^{[86,87]}$ or Parkinson's disease. ${ }^{[88]}$

One of the natural substrates of tyrosinase is tyrosine, which is oxidized by tyrosinase to produce dopaquinone (Fig.1.15). The latter compound either cyclizes to cyclodopa, which leads to eumelanin (black/brown pigment) or reacts with cysteine to give cysteinyldopa resulting in pheomelanin (red/yellow pigment). Afterwards, eumelanin and pheomelanin polymerize in different ratios according to genetic phenotype and give mixed-type melanins. 
<smiles>[R10]CC(C)Oc1ccc(CC(N)C(=O)O)cc1O</smiles>

Tyrosine

Dopa

Dopaquinone

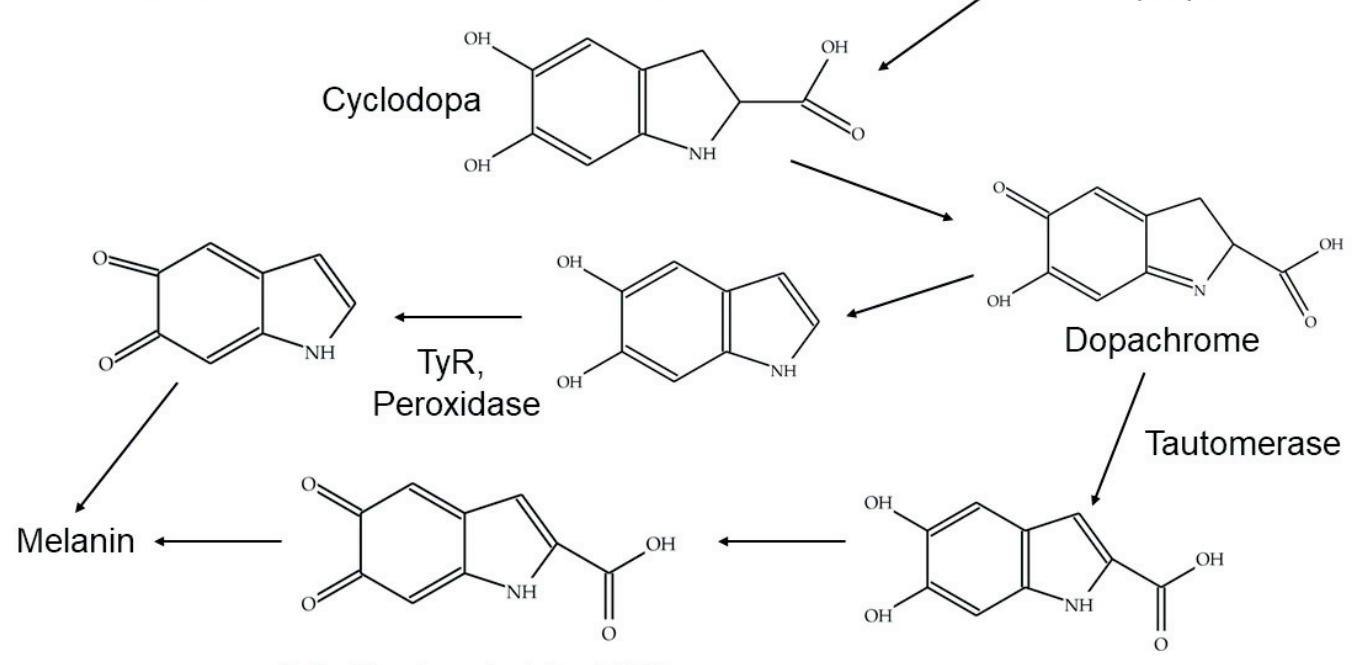

5,6-dihydroxyindole (DHI)

Fig.1.15 Schematic representation of the reactions of L-3,4-dihydroxyphenylalanine (DOPA) catalyzed by tyrosinase.

For melanoma studies, it was shown that tyrosinase is a more sensitive biomarker compared to other protein biomarkers such as HMB-45 and MART-1. ${ }^{89]}$ The commercial monoclonal antibody T311 (provided by Abcam) binds to tyrosinase and has been found to be $94 \%$ sensitive for melanomas, but the sensitivity was correlated inversely with clinical stage. In the clinical stages I and II 100\% immunopositivity was shown. However, stage III and stage IV showed $86 \%$ immunoreactivity each. ${ }^{[90]}$ T311 staining also changed with clinical stages, from an exclusively homogeneous pattern in early stages to a more heterogeneous pattern in later stages. ${ }^{[90]}$ Thus, T311 allows detection of melanoma-associated tyrosinase protein expression and through recognizing tyrosinase derived epitopes, the clinical stages can be diagnosed and distinguished. Therefore, monitoring the spatial localization of tyrosinase on suspected malignant tissues will not only provide earlier and more reliable tumor detection, but also a better understanding of the spatial distribution of special biomarkers, the metabolic state of the tumor and perhaps overcome limitations related to fluorescence quantifications. 


\subsubsection{Melanoma diagnosis and detection methods}

Clinically, dermatologists usually examine skin moles or suspicious skin areas by searching “ABCDE" signs for early recognition of melanoma. ${ }^{[91]}$ ABCDE represents the characteristics of Asymmetry, Border irregularity, Color variegation, Diameter greater than $6 \mathrm{~mm}$ and Evolving (i.e. lesions that have changed over time). If the lesion has the $\mathrm{ABCDE}$ characteristics, a further examination must be done. However, even with dermoscopy, malignant melanoma in its early stages sometimes can be difficult to be distinguished from innocuous skin moles. Traditional ABCDE features may not be present.

To further confirm the malignant skin cancer, a piece of skin lesion will be removed to perform a skin biopsy, and the skin sample will be sent to a pathologist to render a microscopic diagnose. Staining processes for selectively imaging cancer biomarkers or specific genes are necessary to reveal cancer cells under microscopy. Commonly used tests include immunohistochemistry (IHC), ${ }^{[92]}$ fluorescence in situ hybridization (FISH) ${ }^{[93]}$ or comparative genomic hybridization $(\mathrm{CGH}) .{ }^{\left[{ }^{[94]}\right.} \mathrm{IHC}$ exploits the principle of antibodies conjugated with enzymes for the specific binding to the antigens in biological tissues. The enzyme conjugated to the antibody can be peroxidase, which catalyses a color-producing reaction. Thus, the colorful enzymatic products can be observed under an optical microscope. FISH is a molecular cytogenetic technique that uses fluorescent probes that bind to a target DNA or RNA (e.g. mRNA, lncRNA and miRNA) sequence in cells, circulating tumor cells, and tissue samples. Unfortunately, there are some drawbacks in IHC particularly when staining melanoma. The main interference comes from melanin which shows a dark color similar to 3,3'diaminobenzidine (DAB), a universally used chromogen in IHC. ${ }^{[61]}$ Fig.1.16 shows the interference from melanin in a melanoma sample when performing IHC. In Fig.1.16a, the tyrosinase inside human skin slices was labeled with primary Abs and HRPconjugated secondary Abs and DAB was used as the chromogen. In Fig.1.16b, no antibodies were applied. The brown color in Fig.1.16b resulted exclusively from melanin, because no immunostaining has been performed. Therefore, the brown color in Fig.1.16a cannot exclusively be adjudicated to the presence of tyrosinase, but also to the melanin coloration. Another widely employed staining method is FISH. However, it could be affected by autofluorescence and photobleaching, leading to wrong diagnosis. In addition, fluorophore labels on the biomarkers are often toxic, unstable 
and require expensive and sophisticated equipments to measure the fluorescence signals.
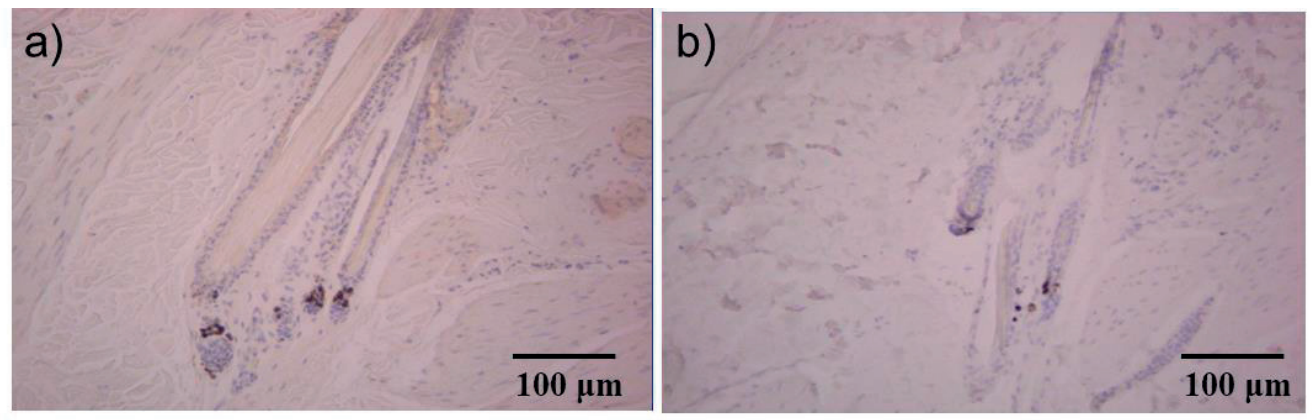

Fig.1.16 a) Optical microscopic image of an immunostained normal skin tissue for tyrosinase detection. b) Optical microscopic image of a normal skin tissue without tyrosinase immunostaining showing the presence of melanin.

In order to improve the traditional melanoma diagnosis, different modalities were developed to enhance the clinical examination quality. For instance, dermoscopy with computer-based analysis, confocal scanning laser microscopy, multispectral digital dermoscopy, and optical coherence tomography are optical instruments for analyzing pigmentation patterns of suspicious melanoma areas. ${ }^{[95,96]}$ Interestingly, daily used hand held devices with high resolution cameras, such as smartphones, can be employed using software that can process the analysis algorithms for the comparison of suspicious skin moles and melanoma images based on a database and thus can help to determine malignancy. ${ }^{[97]}$ Despite the versatility of these optical modalities, the pigmentation can only represent a part of the melanoma characteristics. Thus, these optical methods are not accurate enough for all the melanoma diagnosis because the molecular information such as biomarker distribution is also very important for clinical diagnosis. ${ }^{[98]}$

In contrast, electrochemistry provides efficient tools for the monitoring of malignant tumors, while avoiding common limitations found in optical techniques. ${ }^{[12,99]}$ Electrochemical biosensors have the advantages of low detection limits, wide linear response ranges, and accessibility of quantifying the analytes. Several electrochemical techniques have been reported for melanoma detection. Melanoma biomarkers such as tyrosinase $,{ }^{[100]} \mathrm{S} 100 \mathrm{~B},{ }^{[99]} \mathrm{CD} 146,{ }^{[101]}$ and melanocortin 1 receptor antigen $(\mathrm{MC} 1 \mathrm{R})^{[102]}$ can be detected electrochemically. It was also reported that electrochemical detection of the ratio of L-3,4-dihydroxyphenylalanine (L-DOPA) and tyrosine can indicate the aggressiveness of malignant melanoma. ${ }^{[103]}$

Although SECM has been applied to different biological systems, SECM studies 
of tissues are still under exploration. In most of the cases, the samples which were scanned were "micro tissues" not real tissues from animals. For instance, Sridhar et al. monitored the respiratory activity of 3D cellular aggregates or "micro tissues" by SECM ${ }^{[18]}$ In their approach, microtissues were grown on a Petri dish in situ and the oxygen consumption or the osteogenic differentiation were monitored. ${ }^{[17]}$ Nevertheless, the growing conditions of microtissues and real tissue inside animal bodies are very different. Compared to microtissues, tissues directly from patients are representative and provide a higher reliability for clinical studies.

\subsection{Bio-application of graphene-based nanomaterials}

Graphene-based nanomaterials have attracted tremendous attention since they have several advantages, such as low toxicity, high bio-compatibility for tissue engineering, medical bionic devices and bioelectric sensors. ${ }^{[104-106]}$ Graphene is composed of two-dimensional (2D) honeycomb monolayer $s p^{2}$ hybridized carbon atoms in a plane. ${ }^{[107]}$ The free $\pi$ electrons in graphene provide reactive sites for surface reactions and thus graphene can be chemically modified or oxidized, forming watersoluble derivatives. ${ }^{[108]}$ Graphene oxide (GO) is a highly oxidized form of chemically modified graphene, which consists of $s p^{2}$ and $s p^{3}$ carbon domains with hydroxyl $(-\mathrm{OH})$ and epoxide ( $-\mathrm{O}-$ ) functional groups on the two accessible sides, and carboxylic acid $(-\mathrm{COOH})$ groups at the edges. ${ }^{[106,109]}$ In general, GOs families have better dispersibility, amphiphilicity and functionalizability. Furthermore, the versatility of GO also makes it a popular drug carrier facilitating drug delivery that enables the increasing of the local concentration of therapeutic molecules, promotion of cellular uptake, and enhance the efficiency of photo-thermal therapy. ${ }^{[110-112]}$ This represents a promising strategy for targeting dangerous diseases such as cancer, virus infection, as well as for repair brain injury. ${ }^{[113-115]}$ Other members of the graphene family, such as reduced graphene oxide (rGO), graphene quantum dots (GQDs) and their derivatives were also extensively explored in fields of biosensors, drug delivery, bioimaging, and theranostics. Since GOs have been widely used as a drug carrier and a label for tissues and cell cultures, there are several imaging techniques that can study the update, accumulation and excretion of graphene oxides in animals. Mass spectrometry imaging (MSI) has been one of the 
popular tools. For instance, Huang et al. modified aptamer functionalized $\mathrm{Au}$ nanoparticles on graphene oxide for cancer marker mapping in mouse tissue. ${ }^{[16]}$ Through the detection of characteristic fingerprints in Raman spectra, Raman bioimaging of graphene derivatives is commonly used. ${ }^{[117,118]}$ However, the inherent Raman intensity of graphene derivatives is usually too weak so it requires the enhancer for Raman signal amplification for bio-imaging. ${ }^{[117]}$ Conjugating fluorescent molecules or doping specific elements to increase fluorescence are methods to visualize graphene derivatives in bio-structures. ${ }^{[17]}$ However, tedious doping procedures, fast photobleaching and self-auto fluorescence limit their application. To summarize current methods for the detection of graphene derivatives in biological structures, in most cases the labels attached to the graphene derivates were detected, not the graphene derivative itself. $^{[119]}$ The conjugation between graphene derivative and labels or enhancers might be weakened especially in tissues or cells, resulting in false positive signals. Only by detecting labels, it is also difficult to judge whether graphene derivatives are degraded in the tissues or not. Moreover, the resolution of popular bio-imaging tools such as Positron Emission Tomography/Computed Tomography (PET/CT) is close to $1 \mathrm{~mm}$, which is not high enough to reveal the important details in animals.

SECM imaging of GO based nanomaterials has been reported recently. The imperfections in graphene that disrupt the $s p^{2}$ conjugation can be imaged by SECM in feedback mode. ${ }^{[120]}$ The single flakes of GO also have been visualized by Rapino's group using SECM. ${ }^{[121]}$ The charges present on the surface of a monoatomic GO layer can hinder the SECM tip current or enhance it. Hence, GOs coated on different substrates can be identified in different redox mediators. Interestingly, the current contrast of GO and substrate in the images depends on the electrostatic interactions between the molecular redox mediator and the charges of the sheet surface. Despite that there are a lot of studies about GOs using SECM, the SECM of GOs in animal tissues has never been done before. This is due to the limitations of conventional instrumentation. In this thesis, the soft probes enable the label-free exploration of the GO related materials in the mouse tissues and this will improve the understanding of the in vivo fate of these nanomaterials. 


\subsection{Thesis outline}

This thesis aims at applying Soft-Probe-SECM for the bioimaging of human thick and thin tissues, mouse organs and tissues, and fruit samples. The information from the SECM images can be used for the investigation of the most dangerous form of skin cancer, i.e. melanoma, as well as nanoparticles distributions in the animal tissues and the antioxidants in apple peels. The experiments parts present the chemicals used in this thesis and the experimental procedures of the study. In Chapter 3, tyrosinase on PVDF membrane can be detected electrochemically by taking advantage of its enzymatic activity or its molecular recognition with a tyrosinase antibody. Three tyrosinase detection strategies were developed. In Chapter 4, Soft-Probe-SECM was used for monitoring tyrosinase expression in non-metastatic and metastatic melanoma tissues. SECM can overcome the limitations of optical methods, meanwhile, improved diagnosis and understanding of the spatial distribution of tyrosinase in different melanoma stages was achieved. Chapter 5 presents a non-invasive electrochemical strategy for mapping the antioxidant (AO) activity of apple peels, which counterbalances oxidative stress caused by various external effectors. The global AO activity in the apple peel including lenticels and regions with artificially degraded AOs were mapped using the Soft-Probe-SECM. In Chapter 6, the conductive graphene oxide nanoribbons (GONR) accumulated in the mouse liver were studied by SECM. This is also the first time the SECM was used to scan thick tissue sections. Chapter 7 is devoted to the characterization and implementation of the newly developed "spider probe" which contains eight parallel electrodes that can move independently from each other. The last chapter describes the future perspectives and the general conclusion. 


\section{Chapter 2. Experimental part}

All the chemicals were of analytical grade and used as received. They are listed in Annexe 2.1. The phosphate-buffered saline (PBS) (10 mM, pH 7.4) was composed of $\mathrm{NaCl} 137 \mathrm{mM}, \mathrm{KCl} 2.7 \mathrm{mM}, \mathrm{Na}_{2} \mathrm{HPO}_{4} 10 \mathrm{mM}$ and $\mathrm{KH}_{2} \mathrm{PO}_{4} 1.8 \mathrm{mM}$. Washing and blocking buffers of $1 \%(\mathrm{v} / \mathrm{v})$ BSA were prepared in PBS. The substrate solution for the enzymatic reaction during SECM experiments was made in $0.1 \mathrm{M}$ citrate-phosphate buffer, $\mathrm{pH}=5.0$, and contained additionally commercial TMB and $\mathrm{H}_{2} \mathrm{O}_{2}$ of unknown concentration (not specified by manufacturer).

\subsection{Preparation of GONRs, PEG-GONRs and PEG-MNP-}

\section{GONRs}

GONRs of $\sim 0.8 \mu \mathrm{m}$ size were synthesized and kindly provided by Prof. ChiaLiang Sun and Prof. Yu-Jen Lu (Chang Gung University and Chang Gung Memorial Hospital). ${ }^{[122]}$ Their synthesis was in brief: MWNTs were unzipped by using the microwave assisted synthesis approach followed by chemical oxidation using $\mathrm{KMnO}_{4}$. The crude GONRs were purified by using a PTFE membrane filter $(0.1 \mu \mathrm{m})$ and then re-suspended in water. The D-band and G-band intensities were 2997 and 3191, respectively, resulting in a $I_{\mathrm{D}} / I_{\mathrm{G}}$ ratio of 0.94 (data provided by Sun and $\mathrm{Lu}$ ).

GONRs coated with magnetic $\mathrm{Fe}_{3} \mathrm{O}_{4}$ nanoparticles (MNPs) were synthesized by the chemical co-precipitation method as described elsewhere. ${ }^{[123]}$ In brief, GONRs, $\mathrm{FeCl}_{3} \cdot 6 \mathrm{H}_{2} \mathrm{O}$ and $\mathrm{FeCl}_{2} \cdot 4 \mathrm{H}_{2} \mathrm{O}$ (molar ratio 1:1:2) were mixed in water and sonicated for 30 min under nitrogen purging to facilitate the synthesis of magnetic $\mathrm{Fe}_{3} \mathrm{O}_{4}$ and avoid $\alpha-\mathrm{Fe}_{2} \mathrm{O}_{3}$ formation, which is a product in the presence of oxygen. After purging for 30 min, the temperature was raised to $65^{\circ} \mathrm{C}$. After one hour, $\mathrm{NaOH}$ was added into the system and allowed to react for $30 \mathrm{~min}$ to facilitate the co-precipitation. The MNPGONRs were purified by using magnetic separation and washing three times with water.

PEG was used as a coating reagent for the GONRs and MNP-GONRs to increase the stability. i.e., to prevent aggregation in blood, and to reduce non-specific adsorption. The ratio of PEG and MNP-GONRs was 1:5 (w/w). The GONRs were modified by 
adding the PEG into the GONR solution and sonicating the mixture for $30 \mathrm{~min}$ before injection.

\subsection{Sample preparations}

\subsubsection{Immobilization of biological and nanomaterials on polyvinylidene difluoride (PVDF) membranes}

\section{Preparation of PVDF membranes}

Squared polyvinylidene difluoride (PVDF) membranes $\left(c a .1 \mathrm{~cm}^{2}\right)$ were first wetted in methanol between 5 to 10 seconds and then transferred into water for about 1 min. Then, the PVDF membranes were taken from the water bath and the excess of water was removed carefully with a filter paper.

\section{Immobilization of proteins on PVDF membrane}

$0.8 \mu \mathrm{L}$ of a protein solution $(2 \mathrm{mg} / \mathrm{mL})$, e.g., tyrosinase, was deposited onto a preprepared PVDF membrane by using a calibrated $2.5 \mu \mathrm{L}$ micropipette (Eppendorf). To avoid the formation of any significant topographic feature on the surface of the PVDF membrane, special care was taken that the tip of the pipette was never in physical contact with the PVDF membrane during the protein deposition process. After the deposition, the immobilized protein spot on the PVDF membrane was dried under a gentle stream of nitrogen. The membrane was then placed on a flat glass slide and covered by a Teflon cup to form an electrochemical cell.

If an immunoassay was performed, e.g., for the detection of tyrosinase, on the PVDF membrane for the detection of the specific protein, further experimental procedures were used as follows. A solution of $1 \%$ bovine serum albumin (BSA) in 50 $\mathrm{mM}$ phosphate buffer was employed to block the protein-free regions on the PVDF membrane. Afterwards, the BSA solution was removed and a $\sim 0.2 \mathrm{mg} / \mathrm{mL}$ solution of primary antibody was added to the system and incubated for $30 \mathrm{~min}$. Then, the system was washed with PBS solution $(\mathrm{pH}=7.4)$. Furthermore, a $\sim 0.2 \mathrm{mg} / \mathrm{mL}$ solution of secondary antibody labeled with horse radish peroxidase (HRP) was added and incubated at room temperature for another $30 \mathrm{~min}$. Finally, the system was washed 
twice with the PBS.

\section{Micro-contact printing of a banana cross section}

A fresh banana cross section $(c a .0 .5 \mathrm{~mm} \times 0.5 \mathrm{~mm})$ was cut with a scalpel blade and washed with DI water. A PVDF membrane was brought in contact with the banana piece for around $1 \mathrm{~min}$ in order to transfer biological materials onto the membrane (Fig.2.1) After the membrane with the banana stamp was completely dried, a solution of $50 \mathrm{mM}$ phosphate buffer $(\mathrm{pH}=6.0)$ was employed for washing. The PVDF membrane was then fixed on a microscope glass slide and placed on the bottom of an electrochemical cell that was completed by a Teflon body. This procedure enclosed the sample into a given volume and where the CE and QRE were placed.
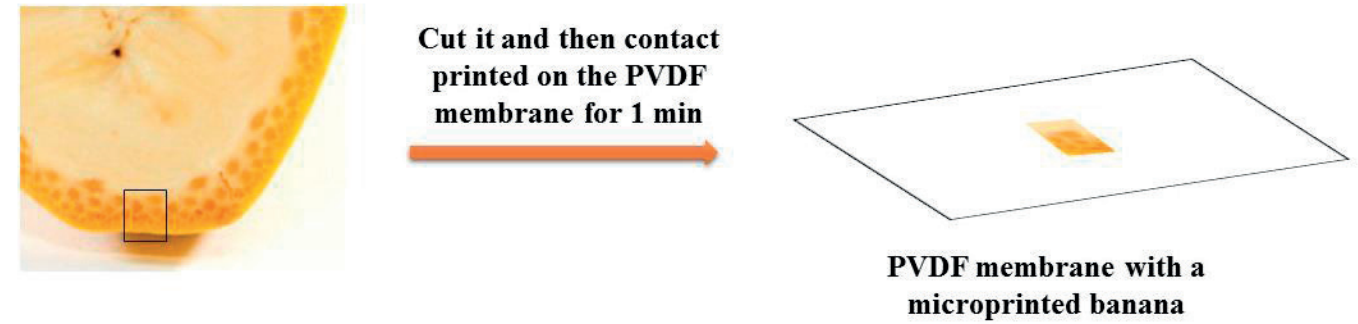

Fig.2.1 Schematic representation for the preparation of microcontact-printed banana on PVDF membranes.

\section{Micro-contact printing of apple cross section}

The apple cross section was cut from a cleaned apple with a scalpel blade and then put in contact with the PVDF membrane for 15 min. Thereafter, the PVDF membrane was placed after a short drying period into the electrochemical cell for SECM experiments.

\section{Spotting nanomaterials on PVDF membrane}

Solutions with different concentrations of nanomaterials (e.g., GONRs) were prepared. Then, $0.8 \mu \mathrm{L}$ of each nanomaterial solution was deposited on the membrane. One membrane can adsorb various parallel nanomaterial spots carefully separated from each other in order not to overlap. After the nanomaterial containing droplets dried completely, they were placed into an electrochemical cell for SECM experiments. 


\subsubsection{Preparation of tissue, organ and fruit samples}

\section{Protocol for the immunodetection of tyrosinase in thin tissues}

Antigen retrieval was done in $10 \mathrm{mM}$ citrate buffer at $95{ }^{\circ} \mathrm{C}$ for $20 \mathrm{~min}$ and followed by cooling at room temperature for $15 \mathrm{~min}$. Finally, the tissues were incubated for $5 \mathrm{~min}$ in PBS buffer. The endogenous peroxidase activity was blocked by $10 \mathrm{~min}$ incubation of the samples in $3 \%(\mathrm{v} / \mathrm{v}) \mathrm{H}_{2} \mathrm{O}_{2}$ in PBS at room temperature (RT). The blocking solution was applied for one hour at RT, and the residual fluid was completely removed. Thereafter, the solution of primary Abs against tyrosinase (100 times diluted in PBS) was added to the samples, incubated for one hour at RT and washed three times with PBS by placing the tissue in the buffer for $5 \mathrm{~min}$. Finally, the slices were immersed in Abs-HRP (100 times diluted in PBS), incubated for one hour at RT and washed with PBS as in the previous step. The slices were placed in an electrochemical cell.

\section{Protocol for the immunodetection of tyrosinase and S100 protein in thick tissues}

Before the immunoassay, the tissue sections were de-paraffinized by immersing them twice in xylene for 40 minutes and rehydrated by immersing them sequentially in solutions of ethanol in water $(100 \%, 95 \%, 70 \%$ and $30 \%$ of ethanol) $(\mathrm{v} / \mathrm{v})$ for $1 \mathrm{~h}$ each. Antigen retrieval was done in $10 \mathrm{mM}$ citrate buffer, $95^{\circ} \mathrm{C}, 40 \mathrm{~min}$, and followed by cooling at RT for $10 \mathrm{~min}$. Finally, the tissues were incubated for $30 \mathrm{~min}$ in PBS. The endogenous peroxidase activity was blocked by $30 \mathrm{~min}$ incubation of the samples in $3 \%(\mathrm{v} / \mathrm{v}) \mathrm{H}_{2} \mathrm{O}_{2}$ in PBS at RT. The blocking solution containing $1 \%$ of BSA in PBS was applied for overnight at $4{ }^{\circ} \mathrm{C}$, and the residual fluid was completely removed. Thereafter, the solution of primary Abs against tyrosinase or S100 proteins (20 times diluted in PBS) was added to the samples, incubated overnight at $4{ }^{\circ} \mathrm{C}$ and washed three times with PBS by placing the tissue in the buffer for one hour at $4{ }^{\circ} \mathrm{C}$. Finally, the slices were immersed in Abs-HRP (20 times diluted in PBS), incubated overnight at $4{ }^{\circ} \mathrm{C}$ and washed with PBS as the previous step described. The slices were placed in an electrochemical cell and the presence of tyrosinase was investigated by SECM using the sample generation-tip collection mode by the detection of $\mathrm{FcMeOH}^{+}$generated enzymatically at Abs-HRP. 
Mice were obtained from the National Laboratory Animal Center (Taiwan) and samples prepared by Prof. Yu-Jen Lu. All experiments were performed within one month after the mice were born. The organs of the mice were taken out 2 hours after the nanoparticle injection. Afterwards, the organs were sealed in paraffin and sectioned into thin or thick slices.

For rehydration, the thin tissue sections were de-paraffinized by immersing them twice in xylene for $10 \mathrm{~min}$ and sequentially in ethanol-water mixtures (100\%, 95\%, $70 \%$ and $30 \%$ of ethanol (v/v)) for 5 min each. The thick tissue sections were deparaffinized followed by the same procedure but with $30 \mathrm{~min}$ of reaction time for each step. After de-paraffinization, the tissue slices were incubated for $30 \mathrm{~min}$ in PBS (10 mM, pH 7.4) composed of $\mathrm{NaCl} 137 \mathrm{mM}, \mathrm{KCl} 2.7 \mathrm{mM}, \mathrm{Na}_{2} \mathrm{HPO}_{4} 10 \mathrm{mM}$ and $\mathrm{KH}_{2} \mathrm{PO}_{4}$ $1.8 \mathrm{mM}$. Finally, the tissues were immersed in the experimental solution for SECM.

\subsubsection{Fruit sample preparation}

A thin slice of apple peel was cut out from the apple with a peeler, placed on a glass slide and cleaned with isopropanol and a soft wipe to remove possible protective layers and other contaminants from previous agricultural, transport and storage treatments. Most importantly, this procedure removed also the highly hydrophobic natural wax layer on the apple peel to allow the permeation of the electrolyte solution into the apple peel. In general, the apple skin consists of a natural epicuticular wax layer (sub- to several micrometer thickness) covering the cuticle $(22-55 \mu \mathrm{m})$, epidermis and hypodermis. ${ }^{[124]}$ The natural wax layer is water repellent and reduces the water as well as hydrophilic AO permeability through the apple skin, but can carefully be removed with organic solvents. ${ }^{[125,126]}$ By using rapidly and gently an isopropanol wetted wipe instead of immersing the apple peel into isopropanol, the natural wax layer was removed and the AO activity was preserved. Previous works have demonstrated that the alcohol based removal of the cuticular wax layer on apples was performed without damaging the epidermal cells. ${ }^{[126]}$ However, the apple peel was quickly covered with a rectangular frame made of Parafilm to expose a fruit peel area of approximately $0.5 \mathrm{~cm}^{2}$ before the immersion of the apple peel in the electrolyte. The electrochemical cell for SECM experiments was completed by a cylindrical Teflon cover mounted on the top and filled with the electrolyte solution (Fig.2.2). 

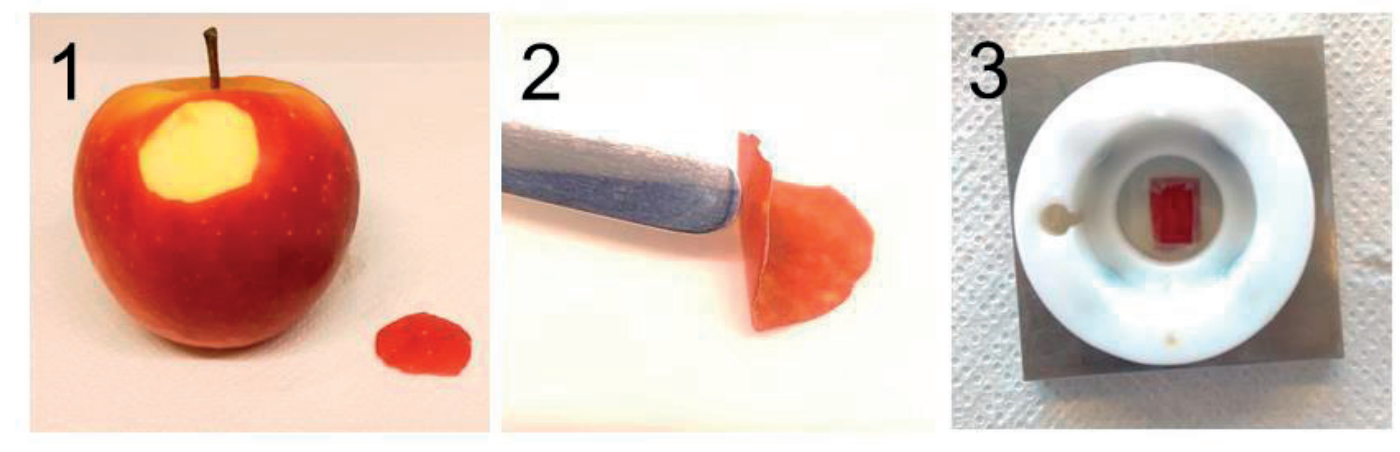

Fig.2.2 Preparation steps of an apple peel sample: 1. The peel was cut with a peeler; 2 .The pulp was removed; 3 . The apple peel was cut into a rectangular piece of about $0.5 \mathrm{~cm}^{2}$ size. The apple peel sample was fixed on a glass slide with a frame made of Parafilm and then a Teflon cup was mounted on the top forming the electrochemical cell.

\subsection{Three-dimensional, inkjet printed test samples}

Three-dimensional test samples were composed of insulating and conductive patterns of various heights and were prepared by using an X-Serie Ceraprinter (Ceradrop). Polyimide (PI, Goodfellow) sheets of $125 \mu \mathrm{m}$ thickness served as substrates and were cleaned with isopropanol prior to printing. A jettable insulator EMD6201 (Sun Chemical) and a carbon nanotube (CNT) dispersion CNTRENE ${ }^{\circledR} 3024$ A3-R (Brewer Science) were subsequently inkjet-printed using $10 \mathrm{pL}$ nominal droplet volume cartridges (Dimatix Fujifilm) to create the insulating and conductive patterns, respectively. The CNTs were mainly double-walled CNTs non-covalently functionalized with a tri-sulfonic acid derivate and have shown good adhesion and electrochemical activity after thermal curing. ${ }^{[127]}$ The insulator was a UV curable dielectric ink that was photo-polymerized simultaneously to printing thanks to a UV LED FireEdge FE300 (380-420 nm; Phoseon Technology) integrated into the corresponding printhead slot of the Ceraprinter. Multilayer printing of the insulator allowed the formation of well-defined three-dimensional microstructures. All printing parameters, such as waveform, jetting frequency and substrate temperature and all postprocessing parameters, such as thermal curing, were optimized. The topography was measured by using a mechanical profilometer Dektak XT from Bruker. 


\subsection{Conventional and Soft-Probe-SECM}

\subsubsection{Preparation of conventional Pt electrode}

Conventional Pt microelectrodes used in this thesis were disk electrodes fabricated by encapsulating a Pt microwire with diameter of $25 \mu \mathrm{m}$ and length of $1 \mathrm{~cm}$ into a glass capillary (outer diameter around $1 \mathrm{~mm}$, thickness of the glass around $50 \mu \mathrm{m}$ ) and sealed with a flame. ${ }^{[128]}$ The Pt wire and the capillary were sealed under high temperature and the other side of the capillary was connected to a vacuum source to ensure a sealing procedure free of gas bubbles (Kopf Vertical Pipette Puller, David Kopf instrument, USA). A copper wire for making the electronic connection part was then inserted into the capillary and connected to the Pt wire by soldering. The tip of the microelectrode was mechanically polished using diamond lapping disks of various roughness to reveal the Pt surface and to adjust the $R G$ value to 5-10.

\subsubsection{Soft stylus probes manufacturing for Soft-Probe-SECM}

The general fabrication principle of the soft probes is described in Chapter 1 . Microchannels were prepared by UV-photoablation (193 nm ArF excimer laser, OPTEC LSV3) in polyethylene terephthalate (PET, Goodfellow) sheets of $100 \mu \mathrm{m}$ thickness. The microchannels were filled with an Electrador carbon ink (Electra Polymer \& Chemicals Ltd.) and after curing at $80^{\circ} \mathrm{C}$ the conductive traces were coated with a Parylene C layer of $2 \mu \mathrm{m}$ thickness (Comelec C-30-S Parylene deposition system). Prior to each SECM experiment, the soft stylus probe was cut manually either with a surgical scalpel blade (Swan-Morton, Sheffield), razor blade or laser assisted cutting to obtain a fresh electrode surface and to achieve a V-shaped tip at the probe of $0.5-2 \mathrm{~mm}$ width with a microelectrode area of $\sim 160 \mu \mathrm{m}^{2}$. However, the electrode size can vary slightly based on the laser ablation process, channel filling procedure and manual cutting result. The cutting procedures were optimized in this thesis and details can be found in the corresponding chapters. For instance, in order to scan samples such as thick tumor sections with Soft-Probe-SECM, the tip of the soft probe was cut with a Spirit 1040-4-SHG laser with $\mu$ FAB Workstation (Spectra Physics - Newport) into a stair case shape providing a small probe-sample contact area for high spatial resolution imaging and maintaining the stable scanning properties of the probe when it is pressed 
against the sample while brushing over it. This type of soft probe will be discussed in detail in Chapter 6.

\subsubsection{SECM instrumentation}

SECM measurements were carried out using a custom-built SECM setup running under SECMx software (G. Wittstock, University of Oldenburg) and comprising an Ivium Compactstat (Ivium Technologies, Netherlands) for single microelectrode measurements or an Ivium CompactStat connected to a MultiWE32 module (Ivium Technologies) for measuring eight microelectrodes in soft arrays simultaneously versus one counter and one reference electrode. A silver wire was used as quasi-reference electrode (QRE) and a platinum wire as the counter electrode (CE). All potentials given herein are referred to the QRE. Conventional SECM experiments were carried out using a platinum microelectrode disk (radius of the Pt disk $12.5 \mu \mathrm{m}$ ) as working electrode. Before each experiment, the platinum microelectrode was mechanically polished with a series of diamond lapping discs starting from $1 \mu \mathrm{m}$ down to $0.05 \mu \mathrm{m}$ particle sizes. The quality of the microelectrode and its $R G$ (i.e. the ratio between the radius of the insulating glass and the radius of the microelectrode $\left(r_{\mathrm{T}}\right)$ ) were determined with a Laborlux D optical microscope (Leitz, Germany). Prior to conventional SECM experiments performed with a rigid Pt microelectrode, the sample surface was leveled by comparing the substrate height (determined by SECM approach curves) at three corners of the area to be scanned. A sample correction angle was applied by using a motorized tilt table (Zaber, USA) on which the electrochemical cell was placed. SoftProbe-SECM experiments were performed with single or arrays of soft microelectrode probes. While the rigid Pt microelectrodes were vertically mounted in a probe holder, the soft probes were placed into a flexible holder that allowed to adjust the probe angle (details and schemes in Chapter 1).

The soft probe was fixed in a special home-made holder to provide a $70^{\circ}$ inclination angle in the probe holder to control the probe bending direction. The soft probe was then brought into mechanical contact with the sample surface until an $h_{p}$ value of several ten to hundred micrometers was achieved. A lift-off routine included into the SECMx software was employed to perform the SECM images. Briefly, the soft stylus probe was kept in contact with the sample surface during forward line scans, in which current values were recorded, while it was operated in a contact-less regime (e.g.. 1000 
$\mu \mathrm{m}$ tip-to-substrate distance) during the relocation of the probe at the starting position of the following forward line scan. In this way, overbending and additional pressure on the probe as well as on the substrate were avoided that would appear when translating the probe back in contact mode.

SECM data analyses were carried out using MIRA software (University of Oldenburg). ${ }^{[129,130]}$ All experiments were performed at room temperature $\left(25 \pm 2{ }^{\circ} \mathrm{C}\right)$.

\subsection{SECM measurements and general measurements}

\section{procedures}

\subsubsection{Indirect SECM detection of adsorbed proteins on PVDF membrane by oxygen reduction method}

After the tyrosinase spot was immobilized on the PVDF membrane and placed inside the electrochemical cell, a solution of $50 \mathrm{mM}$ phosphate buffer $(\mathrm{pH}=6.0)$ was added into the system. Then, the Pt microelectrode was biased at an electrode potential $\left(E_{\mathrm{T}}\right)$ of $-0.8 \mathrm{~V}$ to monitor the reduction of oxygen while scanning the microelectrode closely over the PVDF sample in constant height mode. On the other hand, the oxygen reduction method can be used for a cleaning step to prevent passivation of the electrode. During the oxygen process, the free radicals such as hydroxyl radicals were produced at the tip of the microelectrode. The radicals react with the contaminants on the Pt surfaces, leading to the destruction of the contaminant film and thus a clean Pt surface can be revealed.

2.5.2 SECM detection of tyrosinase activity immobilized on PVDF membrane

L-3,4-dihydroxyphenylalanine (L-DOPA) strategy: After the tyrosine spot was deposited on the PVDF membrane, a solution of $2 \mathrm{mM}$ L-DOPA in $50 \mathrm{mM}$ phosphate buffer $(\mathrm{pH}=6.0)$ was added into the electrochemical cell. Then the Pt microelectrode was scanned close to the sample and biased at $0.7 \mathrm{~V}$ to monitor the tyrosinase activity in constant height mode. 


\subsubsection{Immunoassay strategy}

The SECM read-out of the sandwich immunoassay was performed by adding a commercial solution of $\mathrm{TMB}_{\text {red }}$ and $\mathrm{H}_{2} \mathrm{O}_{2}$, which in presence of HRP produces $\mathrm{TMB}_{o x}$ that can be detected amperometrically at the scanning Pt microelectrode biased at $E_{\mathrm{T}}=$ $-0.3 \mathrm{~V}$.

\subsubsection{SECM images of tyrosinase in thin tissue sections}

For the SECM imaging of tyrosinase in tissue slices deposited on glass slides, both the contact-less (i.e. constant height mode with a conventional Pt microelectrode) and the contact mode (i.e. a soft probe brushing over the surface while keeping $d$ constant) were employed. Approach curves were performed with the Pt microelectrode over the bare glass surface to measure the hindered diffusion current of the oxygen reduction in PBS. By this procedure, the sample tilt was eliminated and the working distance between glass surface and tip was set to $20 \mu \mathrm{m}$. Before SECM imaging, several test line scans were performed to ensure the working distance was larger than the thickness of the tissue. In case the thickness of the tissue slice appeared larger, $d$ was adjusted. Contact mode SECM experiments were performed using a soft carbon microelectrode probe (electrode area about $160 \mu \mathrm{m}^{2}, 0.5-2 \mathrm{~mm}$ width of the $\mathrm{V}$-shaped soft probe tip). The probe was approached on the glass under an inclination angle between the substrate and the carbon microelectrode of $70^{\circ}$. In the beginning of the HRP catalyzed enzymatic reaction, the concentration of $\mathrm{TMB}_{\text {ox }}$ was low and SECM signals were weak and less reproducible. Therefore, in all experiments, special care was taken that the response from the substrate was stable during the time scale (15 min of equilibration) of the experiments in terms of steady-state diffusion layers from the active sites of the tissues. The presence of tyrosinase was established by using the SECM sample generation-tip collection mode through the detection of $\mathrm{TMB}_{o x}$ generated enzymatically at Abs-HRP at $-0.2 \mathrm{~V}$. FcMeOH can replace TMB as the redox mediator for immuno detection and appeared more stable than TMB in the experiments performed in this thesis. Following this detection strategy, $2 \mathrm{mM}$ of $\mathrm{FcMeOH}$ and $1 \mathrm{mM}$ of $\mathrm{H}_{2} \mathrm{O}_{2}$ were added to the experimental solution. When applying $-0.2 \mathrm{~V}$ at the microelectrode using SG/TC mode, $\mathrm{FcMeOH}^{+}$produced by HRP can be detected and thus resulted in an increased current. 


\subsubsection{Immunohistochemistry}

Before the immunoassay, the tissue slices were de-paraffinized by immersing them twice in xylene for $10 \mathrm{~min}$ and rehydrated by immersing them sequentially in solutions of ethanol in water $(100 \%, 95 \%, 70 \%$ and $30 \%$ of ethanol) (v/v) for 5 min each. Antigen retrieval was done in $10 \mathrm{mM}$ citrate buffer, $95^{\circ} \mathrm{C}, 20 \mathrm{~min}$, followed by a cooling down to room temperature for $15 \mathrm{~min}$. Finally, the tissue slices were incubated for $5 \mathrm{~min}$ in PBS buffer. The endogenous peroxidase activity was blocked by $10 \mathrm{~min}$ incubation of the samples in $3 \%(\mathrm{v} / \mathrm{v}) \mathrm{H}_{2} \mathrm{O}_{2}$ in PBS at RT. Subsequently, primary Abs and HRP conjugated secondary Abs were applied. Then, the tissue slices were equilibrated in alkaline phosphatase (AP) buffer ( $\mathrm{pH}$ 9.5), which was composed of 0.1 M Tris base, $0.1 \mathrm{M}$ sodium chloride, and $50 \mathrm{mM}$ magnesium chloride. The tissue sections were covered with nitroblue tetrazolium (NBT) $0.03 \%$ (v/v) and 5-bromo-4-chloro-3-indoly phosphate (BCIP) $0.02 \%(\mathrm{v} / \mathrm{v})$ in AP buffer for $2 \mathrm{~h}$. The tissues were washed with PBS solution and then nuclear fast red (NFR) commercial solution was added in order to stain the nuclei. The tissues were all covered by NFR and stained for $5 \mathrm{~min}$. Subsequently, they were fixed for $10 \mathrm{~min}$ in $4 \%(\mathrm{v} / \mathrm{v})$ paraformaldehyde (PFA) at RT. A quick dehydration was performed in EtOH 70\% (v/v), EtOH 96\% (v/v), 3× EtOH $100 \%(\mathrm{v} / \mathrm{v})$ and xylene. The samples were then covered with proper cover slips.

\subsection{Single-photon emission computed tomography (SPECT)/computed tomography (CT)}

The biodistribution of the PEG-MNP-GONRs was studied in mice by SPECT/CT (NanoSPECT/CT; Bioscan Inc) with a ${ }^{99 m}$ Tc-pertechnetate radiolabel by Prof. Yu-Jen Lu. The mouse experiment was prepared under anesthesia. Scintigraphy was performed immediately after the injection of 2 millicurie (mCi) ${ }^{99 \mathrm{~m}} \mathrm{Tc}-$ labled PEG-MNP-GONRs or ${ }^{99 \mathrm{~m}}$ Tc-labeled PEG-MNP-GONRs into the tail vein. The scintigraphic images were quantitatively analyzed to evaluate the distribution of ${ }^{99 \mathrm{~m}}$ Tc-labled PEG-MNP-GONRs within the systemic circulation. Image intensities were normalized to steady blood pool mean counts around 10 min post-injection for comparison. ${ }^{99} \mathrm{~m}$ Tc-labeled PEG-GONR accumulated at a high level in the liver, relatively high in spleen, and minimal in lung. 
The PEG-MNP-GONRs particles show rapid accumulation in the RES which contains liver Kupffer cells that can rapidly remove foreign particulate materials from circulation. 


\subsection{Annexe 2.1.}

\begin{tabular}{|c|c|c|c|}
\hline Chemical & Supplier & Formula & Chapter \\
\hline $\begin{array}{l}\text { Anti-tyrosinase } \\
\text { monoclonal antibody (Ab) } \\
\text { T311 }\end{array}$ & Bio Medical (USA) & & $3,4,5$ \\
\hline $\begin{array}{l}\text { Bovine serum albumin } \\
\text { (BSA) }\end{array}$ & $\begin{array}{l}\text { Sigma-Aldrich } \\
\text { (Switzerland) }\end{array}$ & & $3,4,5,6$ \\
\hline Catechol & Fluka (Switzerland) & $\mathrm{C}_{6} \mathrm{H}_{4}(\mathrm{OH})$ & 3 \\
\hline Citric acid & $\begin{array}{l}\text { Sigma-Aldrich } \\
\text { (Switzerland) }\end{array}$ & $\mathrm{C}_{6} \mathrm{H}_{8} \mathrm{O}_{7}$ & $3,4,5$ \\
\hline $\begin{array}{l}\text { Commercial 3,3',5,5'- } \\
\text { tetramethylbenzidine } \\
\left(\mathrm{TMB}_{r e d}\right) \text { solution }\end{array}$ & ABRAXIS (USA) & & $3,4,5$ \\
\hline Cripps Pink apples & local market & & 7 \\
\hline $\begin{array}{l}\text { Deionized water Milli-Q } \\
\text { plus } 185 \text { model (produced } \\
\text { on site) }\end{array}$ & $\begin{array}{l}\text { Millipore } \\
\text { (Switzerland) }\end{array}$ & $\mathrm{H}_{2} \mathrm{O}$ & $3,4,5,6,7$ \\
\hline $\begin{array}{l}\text { Dipotassium } \\
\text { monohydrogen phosphate }\end{array}$ & $\begin{array}{l}\text { Sigma-Aldrich } \\
\text { (Switzerland) }\end{array}$ & $\mathrm{K}_{2} \mathrm{HPO}_{4}$ & $3,4,5,6,7$ \\
\hline Ethanol & $\begin{array}{l}\text { Sigma-Aldrich } \\
\text { (Switzerland) }\end{array}$ & $\begin{array}{l}\mathrm{CH} 3 \mathrm{CH}_{2} \mathrm{O} \\
\mathrm{H}\end{array}$ & $4,5,6$ \\
\hline $\begin{array}{l}\text { Ferrocenemethanol } \\
(\mathrm{FcMeOH})\end{array}$ & $\begin{array}{l}\text { Fisher Chemicals } \\
\text { (Switzerland) }\end{array}$ & $\begin{array}{l}\mathrm{Fe}\left(\mathrm{C}_{5} \mathrm{H}_{5}\right)( \\
\left.\mathrm{C}_{5} \mathrm{H}_{4}\right) \mathrm{CH}_{2} \\
\mathrm{OH}\end{array}$ & $3,4,5,6,7$ \\
\hline $\begin{array}{l}\text { Formaldehyde solution } \\
(4 \% \text { in PBS) }\end{array}$ & $\begin{array}{l}\text { Alfa Aesar } \\
\text { (Karlsruhe, } \\
\text { Germany) }\end{array}$ & $\mathrm{H}_{2} \mathrm{CO}$ & $3,4,5,6,7$ \\
\hline Hemoglobin (human) & $\begin{array}{l}\text { Sigma-Aldrich } \\
\text { (Switzerland) }\end{array}$ & & 6 \\
\hline Hydrogen peroxide ( $3 \%$ ) & $\begin{array}{l}\text { Sigma-Aldrich } \\
\text { (Switzerland) }\end{array}$ & $\mathrm{H} 2 \mathrm{O} 2$ & $3,4,5$ \\
\hline Hydrogen peroxide, $60 \%$ & $\begin{array}{l}\text { Fisher Chemicals } \\
\text { (Switzerland) }\end{array}$ & $\mathrm{H} 2 \mathrm{O} 2$ & 7 \\
\hline
\end{tabular}




\begin{tabular}{|c|c|c|c|}
\hline $\begin{array}{l}\text { L-3,4- } \\
\text { dihydroxyphenylalanine } \\
\text { (L-DOPA, 99\%) }\end{array}$ & $\begin{array}{l}\text { Sigma-Aldrich } \\
\text { (Switzerland) }\end{array}$ & $\mathrm{C}_{9} \mathrm{H}_{11} \mathrm{NO}_{4}$ & 3 \\
\hline Methanol ( $\geq 99 \%)$ & Merck (Switzerland) & $\mathrm{CH} 3 \mathrm{OH}$ & $3,4,5,6,7$ \\
\hline $\begin{array}{l}\text { Monopotassium } \\
\text { dihydrogen phosphate }\end{array}$ & $\begin{array}{l}\text { Sigma-Aldrich } \\
\text { (Switzerland) }\end{array}$ & $\mathrm{KH}_{2} \mathrm{PO}_{4}$ & $3,4,5,6,7$ \\
\hline Mouse organ sections & $\begin{array}{l}\text { Chang Gung } \\
\text { Memorial Hospital } \\
\text { (Taiwan) }\end{array}$ & & 5,6 \\
\hline $\begin{array}{l}\text { Multiwall carbon } \\
\text { nanotubes (MWNTs) } \\
\text { (TNSMC1, diameter 6-8 } \\
\text { nm; length = 0.5-2 } \mu \mathrm{m} ;- \\
\text { COOH content = } 3.86 \mathrm{wt} \% \text { ) }\end{array}$ & $\begin{array}{l}\text { Chengdu Organic } \\
\text { Chemicals Co. Ltd. } \\
\text { (Chinese Academy of } \\
\text { Sciences, Chengdu, } \\
\text { China) }\end{array}$ & & 6 \\
\hline $\begin{array}{l}\text { Nitro blue tetrazolium } \\
\text { (NBT) }\end{array}$ & Roche (Switzerland) & $\begin{array}{l}\mathrm{C}_{40} \mathrm{H}_{30} \mathrm{Cl}_{2} \\
\mathrm{~N}_{10} \mathrm{O}_{6}\end{array}$ & 4 \\
\hline $\begin{array}{l}\text { Paraffin-embedded skin } \\
\text { tissue micro-arrays (TMA) }\end{array}$ & $\begin{array}{l}\text { US Biomax, Inc. } \\
\text { (USA) }\end{array}$ & & 4 \\
\hline $\begin{array}{l}\text { Paraffin-embedded thick } \\
\text { melanoma and normal } \\
\text { skin tissues }\end{array}$ & Sofia Bio (Bulgaria) & & 5 \\
\hline Phosphoric acid & J.T. Baker (USA) & $\mathrm{H}_{3} \mathrm{PO}_{4}$ & $3,4,5,6,7$ \\
\hline $\begin{array}{l}\text { Polyethylene glycol (PEG) } \\
\text { (Sunbright DSPE-050-PA, } \\
\text { molecular weight of PEG } \\
\text { chains = 5000) }\end{array}$ & $\begin{array}{l}\text { NOF Cooperation } \\
\text { (Japan) }\end{array}$ & & 6 \\
\hline $\begin{array}{l}\text { Polytetrafluoroethylene } \\
\text { (PTFE) membrane ( } 0.1 \mu \mathrm{m} \\
\text { pore size) }\end{array}$ & $\begin{array}{l}\text { Millipore } \\
\text { (Switzerland) }\end{array}$ & & 6 \\
\hline $\begin{array}{l}\text { Polyvinylidene difluoride } \\
\text { (PVDF) membranes }\end{array}$ & Bio-Rad (USA) & & $3,4,5,6,7$ \\
\hline $\begin{array}{l}\text { Potassium chloride ( } \geq \\
99.5 \% \text { ) }\end{array}$ & Fluka (Switzerland) & $\mathrm{KCl}$ & $3,4,5,6,7$ \\
\hline Potassium permanganate & J.T. Baker (USA) & $\mathrm{KMnO}_{4}$ & 6 \\
\hline $\begin{array}{l}\text { Secondary anti-mouse Abs } \\
\text { conjugated with horse } \\
\text { radish peroxidase (Abs- } \\
\text { HRP) }\end{array}$ & Abcam (UK) & & $3,4,5$ \\
\hline
\end{tabular}




\begin{tabular}{|c|c|c|c|}
\hline Sodium chloride $(99.5 \%)$ & Fluka (Switzerland) & $\mathrm{NaCl}$ & $3,4,5,6,7$ \\
\hline Sodium hydroxide & J.T. Baker (USA) & $\mathrm{NaOH}$ & 6 \\
\hline $\begin{array}{l}\text { Sodium phosphate } \\
\text { monobasic (99\%) }\end{array}$ & Fluka (Switzerland) & $\mathrm{NaH}_{2} \mathrm{PO}_{4}$ & $3,4,5,6,7$ \\
\hline Sulfuric acid & J.T. Baker (USA) & $\mathrm{H}_{2} \mathrm{SO}_{4}$ & 6 \\
\hline $\begin{array}{l}\text { Tris base, } 3,3^{\prime}, 5,5^{\prime}- \\
\text { tetramethylbenzidine } \\
\text { (TMB) powder }\end{array}$ & $\begin{array}{l}\text { Sigma-Aldrich } \\
\text { (Switzerland) }\end{array}$ & $\mathrm{C}_{16} \mathrm{H}_{20} \mathrm{~N}_{2}$ & $3,4,5$ \\
\hline Triton X-100 & $\begin{array}{l}\text { Sigma-Aldrich } \\
\text { (Switzerland) }\end{array}$ & & 4 \\
\hline $\begin{array}{l}\text { Tyrosinase (from } \\
\text { mushroom, lyophilized } \\
\text { powder, >=1000 unit/mg } \\
\text { solid) }\end{array}$ & $\begin{array}{l}\text { Sigma-Aldrich } \\
\text { (Switzerland) }\end{array}$ & & $3,4,5$ \\
\hline $\begin{array}{l}\text { Ruthenium hexaamine } \\
\text { chloride }\end{array}$ & $\begin{array}{l}\text { Sigma-Aldrich } \\
\text { (Switzerland) }\end{array}$ & $\begin{array}{l}\mathrm{Cl}_{3}[\mathrm{Ru}(\mathrm{N} \\
\left.\left.\mathrm{H}_{3}\right)_{6}\right]\end{array}$ & 5,6 \\
\hline Xylene & $\begin{array}{l}\text { Sigma-Aldrich } \\
\text { (Switzerland) }\end{array}$ & $\begin{array}{l}\left(\mathrm{CH}_{3}\right)_{2} \mathrm{C}_{6} \mathrm{H} \\
4\end{array}$ & $4,5,6$ \\
\hline $\begin{array}{l}\text { 3,3'-diaminobenzidine } \\
\text { (DAB) }\end{array}$ & $\begin{array}{l}\text { Sigma-Aldrich } \\
\text { (Switzerland) }\end{array}$ & $\mathrm{C}_{12} \mathrm{H}_{14} \mathrm{~N}_{4}$ & 4 \\
\hline $\begin{array}{l}\text { 5-bromo-4-chloro- } \\
\text { 3indolyphosphate (BCIP) }\end{array}$ & $\begin{array}{l}\text { Fisher Chemicals } \\
\text { (Switzerland) }\end{array}$ & $\begin{array}{l}\mathrm{C}_{8} \mathrm{H}_{6} \mathrm{BrCl} \\
\mathrm{NO}_{4} \mathrm{P}\end{array}$ & 4 \\
\hline
\end{tabular}


tyrosinase in micro-contact printed fruit samples on polyvinylidene fluoride membrane

Adapted from: Tzu-En Lin, Fernando Cortés-Salazar, Andreas Lesch, Liang Qiao, Alexandra Bondarenko and Hubert H. Girault, Electrochimica Acta 2015, 179, 57-64.

\subsection{Introduction}

Tyrosinase is a copper-containing enzyme known for catalyzing the hydroxylation of monophenols and their oxidation to the respective quinones in the presence of oxygen. Furthermore, tyrosinase is directly involved in fruit ripening, in the biosynthesis of the skin pigment melanin and in skin disorders such as vitiligo (i.e. skin depigmentation due to loss of melanin). ${ }^{[131-134]}$ Furthermore, it is a biomarker in melanoma and its expression level is very high in some stages. ${ }^{[79,90,135-137]}$ Therefore, the accurate and sensitive detection of tyrosinase could provide relevant information for a better understanding of different tyrosinase-related biological processes.

The importance of tyrosinase attracts scientists' attention and thus the detection of tyrosinase or its substrates have been widely investigated using various analytical techniques. Most methods for the detection of tyrosinase are optical based assays which take advantages of a color or fluorescence change of a substrate converted by an enzymatic reaction. ${ }^{[138,139]}$ Tyrosinase can be incorporated into electrochemical sensors for the detection of phenolic compounds using amperometric methods. The tyrosinase immobilized on the electrodes can provide selectivity for the detection of neurotransmitters, catechol, coliforms metabolites, and phenols. ${ }^{[140-143]}$ Alternatively, these enzymatic reactions could potentially be used to localize tyrosinase immobilized on substrate surfaces by detecting the reaction products of the enzymatic reaction locally. This is important, because tyrosinase, as well as other enzymes, is normally expressed inside cells or tissues in concentration levels that are temporally and spatially dependent. 
In proteomics, a common detection strategy employed to detect tyrosinase is based on the extraction, separation and identification of proteins by using protein electrophoresis (e.g. isoelectrofocusing (IEF) electrophoresis or sodium dodecyl sulfate polyacrylamide gel electrophoresis (SDS-PAGE)), protein blotting on a suitable membrane and protein identification by using different labeling protocols. ${ }^{[144]}$ Among the different employed membranes for protein blotting and detection, polyvinylidene fluoride (PVDF) is widely used since it is a highly hydrophobic and porous support with a superior protein binding capacity and a remarkable mechanical and chemical stability. Moreover, PVDF maintains the enzymatic activity of adsorbed proteins and is compatible with different protein labeling protocols including those based on Coomassie Blue, silver or gold staining and fluorescent or chemiluminescent dyes. ${ }^{[145-}$ ${ }^{147]}$ Recently, proteins immobilized on PVDF membranes have been employed for the visualization of human finger prints by using SECM. ${ }^{[148]}$ For this purpose, silverstaining, ${ }^{[146]}$ multi-metal-deposition (MMD $)^{[130]}$ or benzoquinone-tagging ${ }^{[130]}$ strategies have been successfully coupled with SECM for the sensitive and selective spatial detection of immobilized proteins. Although these staining methods are powerful, they are time consuming and require the use of expensive dyes. Therefore, developing label free detection methods is attractive.

SECM enables a highly resolved spatial detection either in blotted protein separations or in the original samples based on a specific enzymatic activity or a selective tagged protein while avoiding optical interferences such as sample color background and light scattering. ${ }^{[148-155]}$

In this chapter, three strategies are presented to further extend the capabilities of SECM as a tool for the detection of adsorbed proteins on PVDF membranes. First, an indirect SECM protein detection method based on local oxygen concentration differences between protein-bound and protein-free PVDF regions was implemented. Second, the enzymatic activity of tyrosinase spots immobilized on PVDF membranes was studied using the substrate L-3,4-dihydroxyphenylalanine (L-DOPA). Finally, a third tyrosinase detection method based on the SECM readout of a tyrosinase specific immune reaction was developed adopting an immunoassay. All three strategies were consecutively employed over the same sample region to detect the presence and enzymatic activity of tyrosinase inside banana peels microcontact printed $(\mu \mathrm{CP})$ on a PVDF membrane. Finally, differential pulse voltammetry (DPV) and mass 
spectrometry (MS) analysis were performed in order to unravel the origin of the recorded SECM response.

\subsection{Results and discussion}

\subsubsection{Indirect SECM detection of adsorbed proteins on PVDF membrane by oxygen reduction method}

The location of tyrosinase spots immobilized on a PVDF membrane was determined indirectly through a so-called "oxygen reduction method". ${ }^{[156]}$ This strategy is based on the detection of oxygen at a scanning microelectrode. The concentration of oxygen in the PVDF membrane pores differ significantly between the regions with and without adsorbed proteins (Fig.3.1a). Such difference results from the protein adsorption on the porous PVDF membrane, which locally reduces the hydrophobicity of the PVDF and enables the wetting of the protein-bound PVDF pores that otherwise remain filled with air. The pore wetting is most likely caused by the polar groups of the adsorbed proteins that might mainly be exposed to the center of the pores. As a result, the concentration of oxygen in the pores filled with air (ca. $\left.274 \mathrm{mg} / \mathrm{L}, 25^{\circ} \mathrm{C}, 1 \mathrm{~atm}\right)$ contrasts with the oxygen concentration in the pores filled with adsorbed proteins and water ( $\left.c a .9 \mathrm{mg} / \mathrm{L}, 25^{\circ} \mathrm{C}, 1 \mathrm{~atm}\right)$. Therefore, by amperometrically monitoring the oxygen concentration at the scanning probe (by its electrochemical reduction at $E_{\mathrm{T}}=-$ $0.8 \mathrm{~V}$ ), a clear differentiation between the zones without adsorbed proteins (depicted as higher negative current values or higher normalized currents $\left(I_{\mathrm{T}}\right)$ ) and with adsorbed proteins (depicted as lower negative current values or lower $I_{\mathrm{T}}$ ) can be achieved.

Indeed, it has already been shown that SECM is a powerful tool for the spatial probing of oxygen in diffusion-limited processes as it has already been applied for the monitoring of the oxygen transport through porous materials, such as cartilages and porous membranes. ${ }^{[157,158]}$ Furthermore, SECM has been used for screening oxygen reduction reaction (ORR) catalyst libraries and providing both qualitative and quantitative local catalytic activity information. ${ }^{[159,160]}$ Oxygen consumption methods have been applied for study cell viability and detection of respiratory responses in living cells. ${ }^{[161-164]}$ 
a)

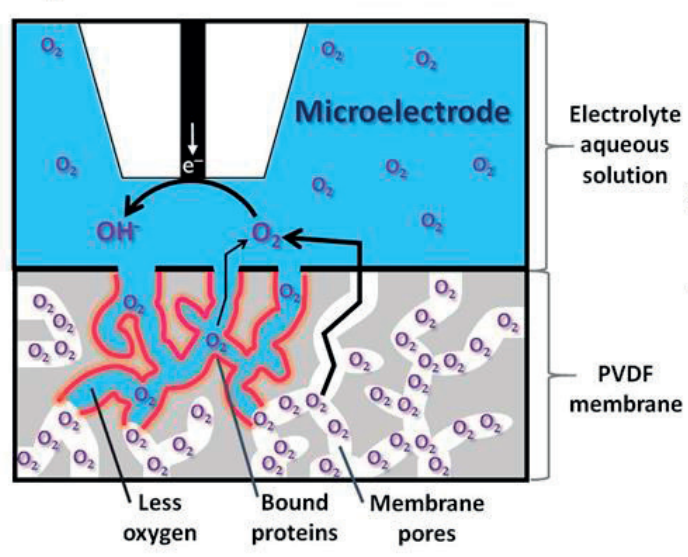

b)

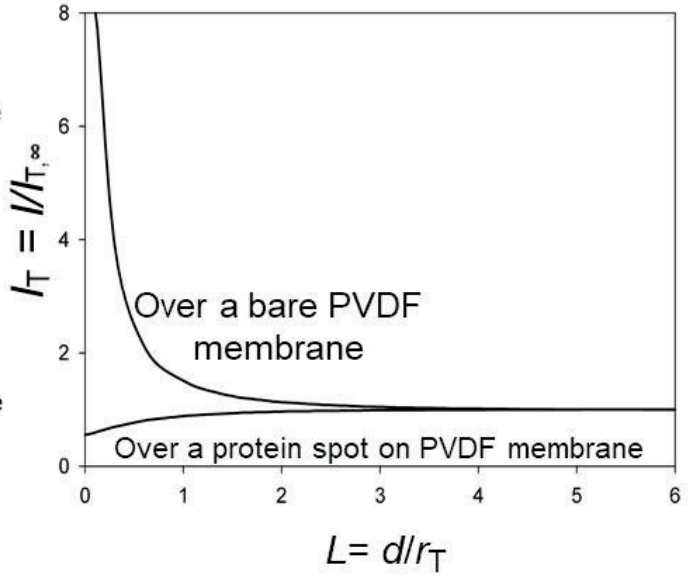

Fig.3.1 a) Schematic representation of the oxygen reduction method for the indirect SECM detection of adsorbed proteins on PVDF membranes. b) SECM approach curves over a PVDF membrane with and without adsorbed proteins in a solution of $0.15 \mathrm{M} \mathrm{KCl}$. Experimental conditions: $R G$ was between 3 to $4 . E_{\mathrm{T}}=-0.8 \mathrm{~V}$, step size $0.5 \mu \mathrm{m}$ and probe translation speed $1 \mu \mathrm{m} / \mathrm{s}$.

The SECM approach curves performed on a protein-free PVDF region with the probe biased at $-0.8 \mathrm{~V}$ show a clear increase of $I_{\mathrm{T}}$ as the concentration of $\mathrm{O}_{2}$ surrounding the air-filled PVDF pores is higher than in solution bulk (Fig.3.1b). Accordingly, a current decrease is observed when the probe was brought to a working distance $(d)$ closer to the protein-bound PVDF membrane, since the concentration of $\mathrm{O}_{2}$ (that is equal inside and outside the protein-bound PVDF pores) is depleted due to its consumption at the microelectrode and the blocking of $\mathrm{O}_{2}$ diffusion towards the microelectrode in the gap between the sample and the probe (Fig.3.1b). It is expected that with such methodology a considerable current contrast between the regions with and without adsorbed proteins will be achieved. For comparison, two protein labeling methods coupled with SECM feedback mode readout were performed for the visualization of the adsorbed proteins from fingerprints, i.e., benzoquinone tagging and silver staining (experimental data were kindly provided by Dr. Fernando CortésSalazar). Fig.3.2 describes the protein detection strategy by the mediated reduction of protein-quinone adducts. The free cysteines in the protein have been tagged with benzoquinone to form protein-quinone adducts. The quinone group undergoes a reduction reaction with $\mathrm{Fe}(\mathrm{CN})_{6}{ }^{4-}$, which is produced at the SECM tip by the electrochemical reduction of $\mathrm{Fe}(\mathrm{CN})_{6}{ }^{3-}$. Consequently, this results in the recycling of 
the redox mediator and contributes to the increment in current signals. Fig.3.4a displays the SECM image of human fingerprints using protein ink deposited on PVDF membrane and with benzoquinone tagging. ${ }^{[165]}$

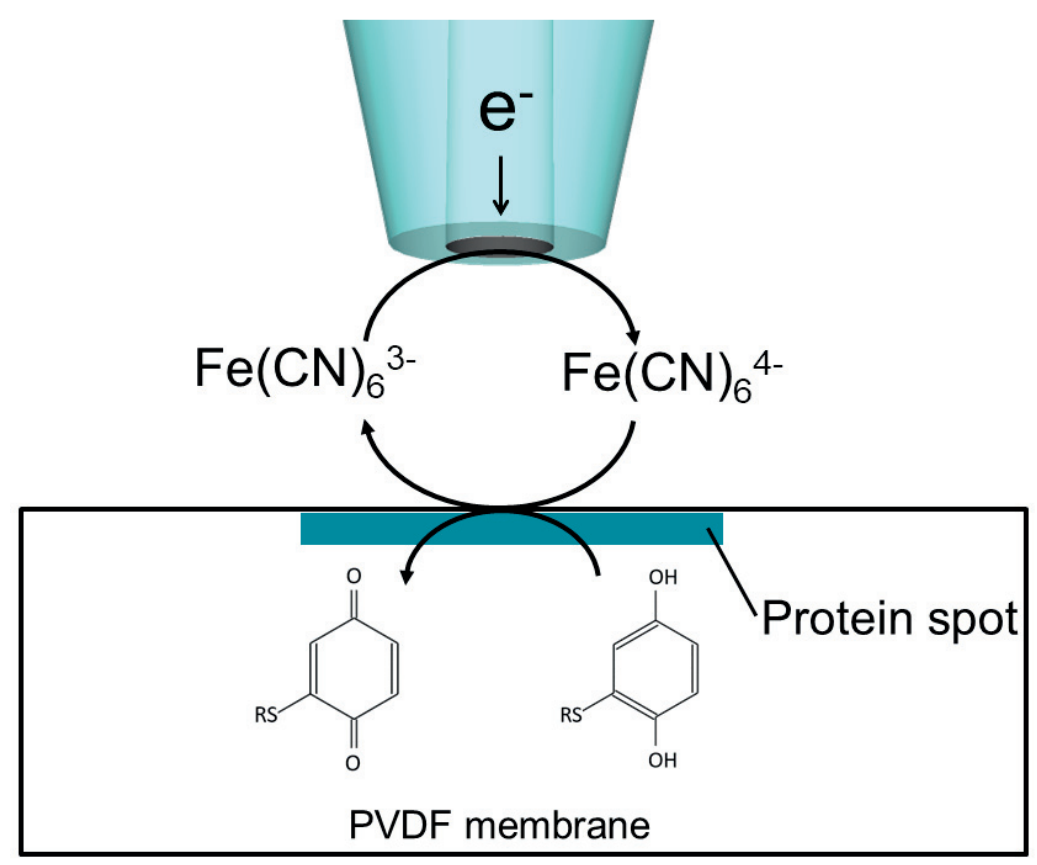

Fig.3.2 Schematic representation of the protein detection principle by the mediated reduction of protein-quinone adducts. ${ }^{[166]}$

Similar to benzoquinone tagging methods, silver staining is commonly used for adsorbed protein detection on the PVDF membrane. ${ }^{[130,167,168]}$ Silver nanoparticles precipitate at the location of the proteins, first by binding $\mathrm{Ag}$ ions that then get chemically reduced, and can be detected. The regeneration of the redox mediator by silver deposited area is expressed in Fig.3.3. Fig.3.4c shows the SECM image of the human finger print based on silver staining. In contrast, Fig.3.4b and $\mathbf{d}$ show the results of SECM imaging applying the oxygen reduction strategy of the same samples. In fact, this concept could compete with the sensitivity of benzoquinone tagging and silver staining, because significant patterns and features of the finger print were identically located. 


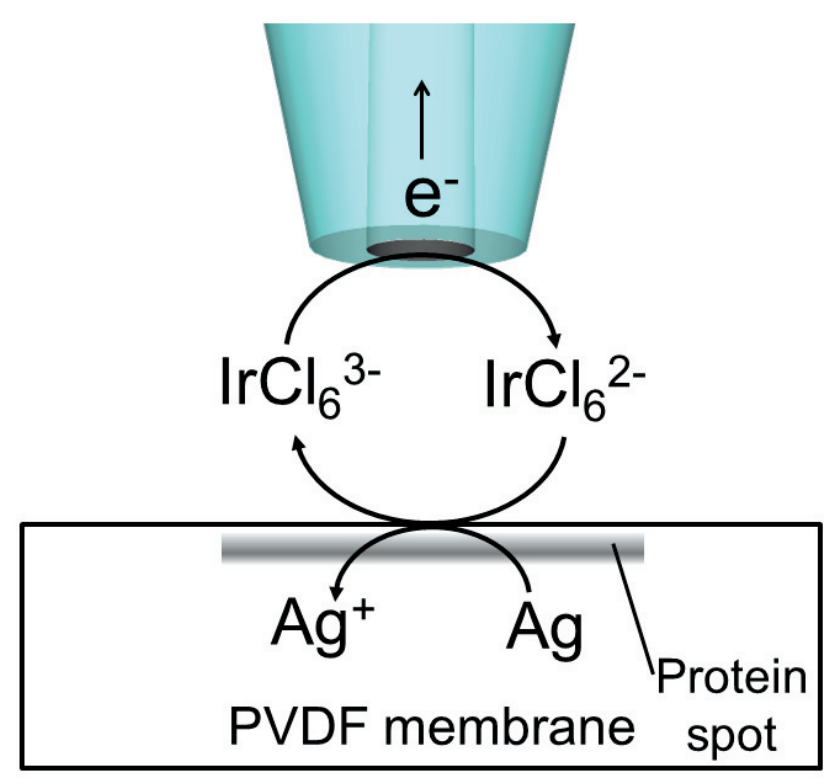

Fig.3.3 Schematic representation of SECM detection strategy of protein with silver staining on the PVDF membrane.

It is important to note that the formation of reactive oxygen species (ROS) can take place during the reduction of oxygen at Pt microelectrodes, as reported elsewhere. ${ }^{[156]}$ Those ROS species have been demonstrated to be able to etch different substrates as elucidated by SECM imaging of the affected areas. In the studied system, it is otherwise less likely that ROS species play a major role on the observed signal since the presence of scavengers (e.g. L-DOPA, vide infra) in the media and the use of a highly stable substrate such as the PVDF membrane reduces certainly such possibility. ${ }^{[169]}$ 

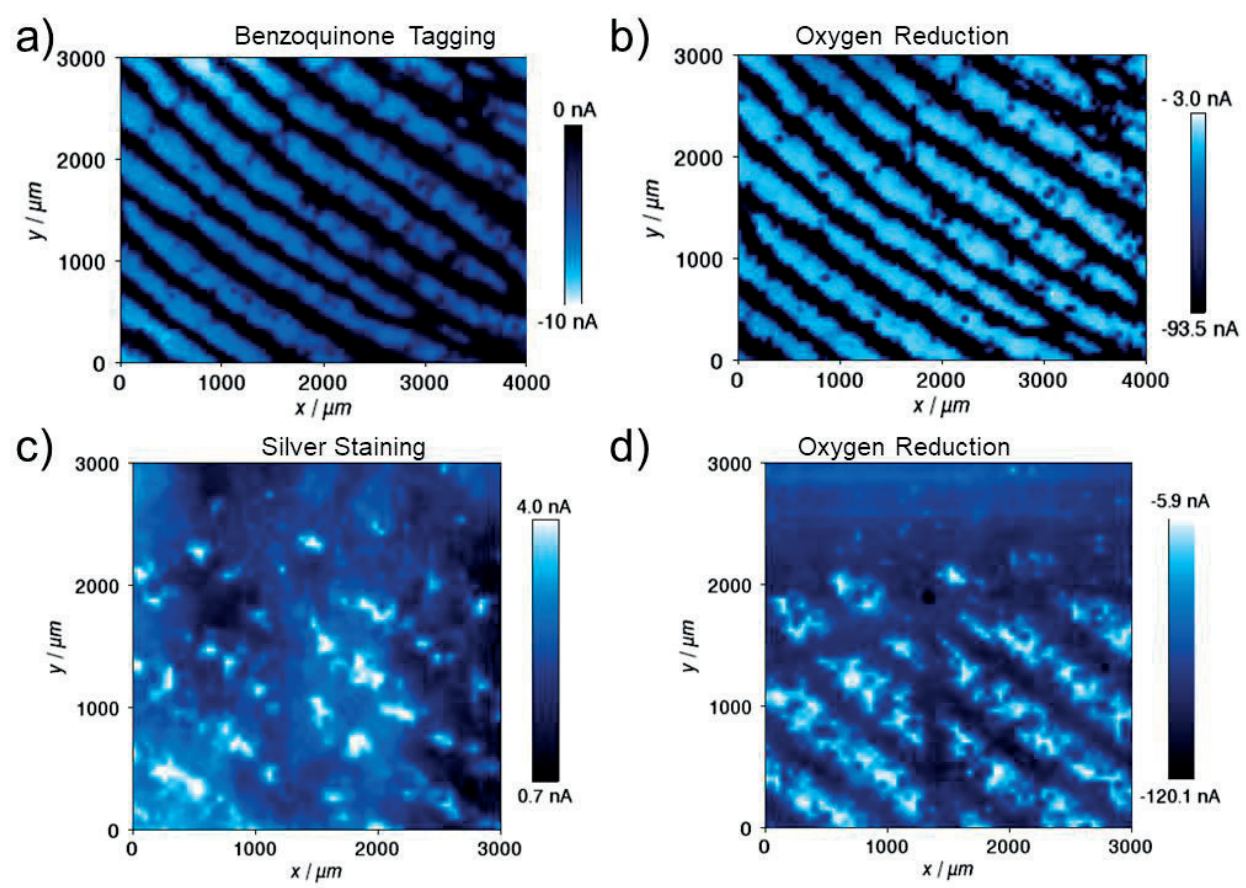

Fig.3.4 Constant height image of human fingerprints on a PVDF membrane imaged by SECM coupled with a) benzoquinone tagging (electrode potential $=E_{\mathrm{T}}=-0.2 \mathrm{~V}$ in $2 \mathrm{mM} \mathrm{K}_{3}\left[\mathrm{Fe}(\mathrm{CN})_{6}\right]$ and $\left.\left.0.1 \mathrm{M} \mathrm{KNO}_{3}\right), \mathrm{b}\right)$ and d) oxygen reduction method $\left(E_{\mathrm{T}}=-0.8 \mathrm{~V}\right)$ and c) silver staining $\left(E_{\mathrm{T}}\right.$ $=0.8 \mathrm{~V}$ in $2 \mathrm{mM} \mathrm{K}_{3}\left[\mathrm{IrCl}_{6}\right], 0.1 \mathrm{M} \mathrm{KCl}$ ). The scanned areas in a) and b), and in c) and d) are identical. Experimental conditions: $d=3 \mu \mathrm{m}$, step size $=50 \mu \mathrm{m}$, translation speed $=50 \mu \mathrm{m} / \mathrm{s}$. This figure was kindly provided by Dr. Fernando Cortés-Salazar.

\subsubsection{Detection of tyrosinase enzymatic activity by SECM}

The enzymatic activity of an adsorbed tyrosinase spot on PVDF membrane was analyzed in a $2 \mathrm{mM}$ solution of L-DOPA (i.e., a natural tyrosinase substrate) in phosphate buffer $(50 \mathrm{mM}, \mathrm{pH}=6.5)$. The enzymatic product of L-DOPA and tyrosinase was detected at the microelectrode and thus induced an increased current over the spot when applying $0.7 \mathrm{~V}$ at the microelectrode (analysis of the mechanism follows vide infra). However, during the imaging process a clear decrease of the current was obtained due to the electrode passivation (Fig.3.5a). Fig.3.5b shows a cyclic voltammogram (CV) of L-DOPA where an almost steady-state signal is recorded for its electrochemical oxidation to dopaquinone. The significant decrease in the observed signal can be explained by electrode fouling taking place during the oxidation of LDOPA at the Pt microelectrode, which is most likely the adsorption of polymerized products. To lower the impact of such passivation during long-term SECM experiments, 
an electrochemical cleaning procedure based on biasing the microelectrode at $-0.8 \mathrm{~V}$ for some seconds in between line scans and hence before applying again the oxidation potential of L-DOPA was implemented. As it can be seen from chronoamperometry (CA) experiments (see Fig.3.5c), the current recorded for the oxidation of L-DOPA (ca., $8 \mathrm{nA}$ ) is recovered almost completely after a potential step of $-0.8 \mathrm{~V}$ has been applied for $20 \mathrm{~s}$. By applying the cleaning steps, significantly longer imaging times were achieved that allowed the complete scanning of the tyrosinase spot (Fig.3.5d). It is important to notice that the efficient electrochemical cleaning of the electrode is achieved only at $\mathrm{pH}$ values around 6 , but not equal or higher than 7 where an even stronger electrode passivation took place impeding any complete SECM mapping of the tyrosinase spot (results not shown). However, the optimum $\mathrm{pH}$ value for tyrosinase activity is around $7,{ }^{[82]}$ thus slower kinetics for the enzymatic reaction are expected.

a)

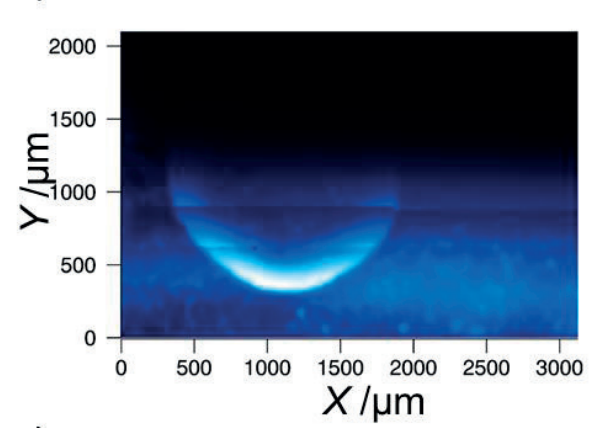

c)

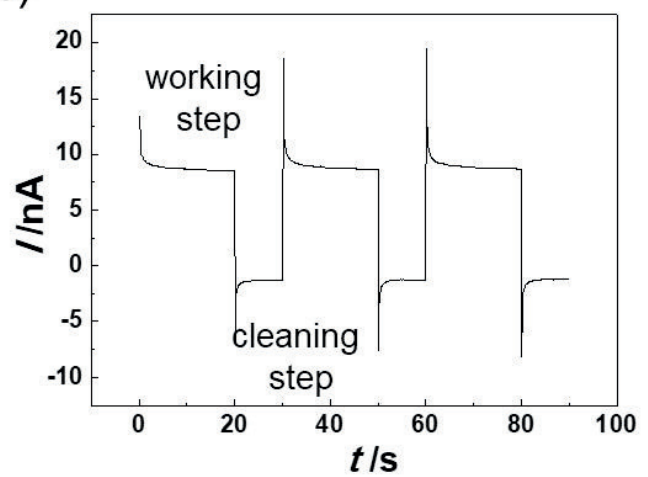

b) 15

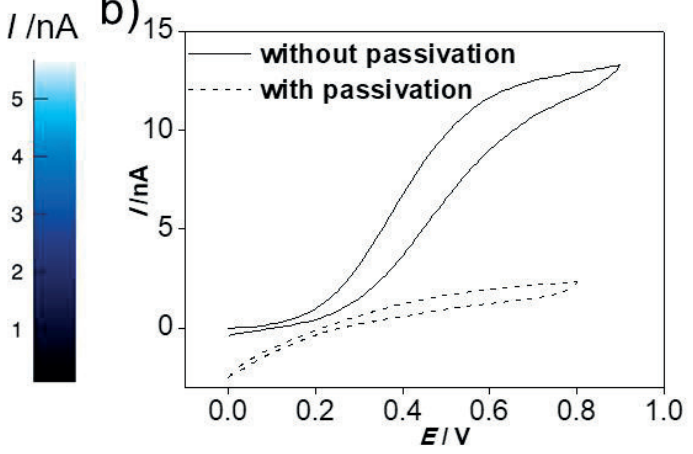

d)

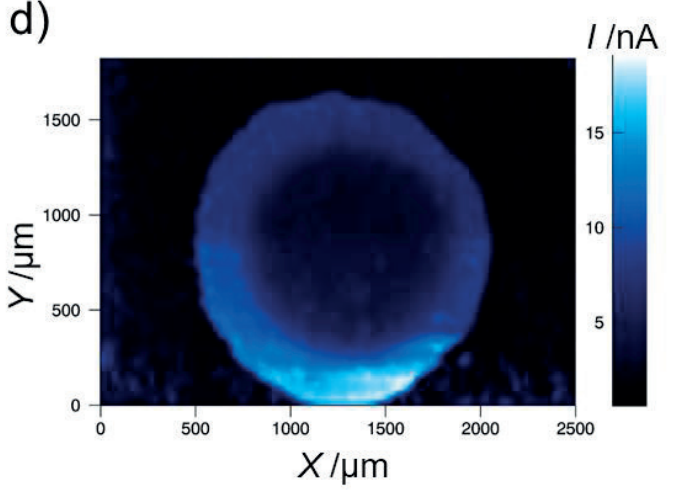

Fig.3.5 SECM image of a tyrosinase spot on a PVDF membrane in $50 \mathrm{mM}$ phosphate buffer (a) without and (d) with electrochemical cleaning. Experimental conditions: Forward $E_{\mathrm{t}}=0.7$ $\mathrm{V}$, reverse $E_{\mathrm{t}}=-0.8 \mathrm{~V}, \mathrm{Pt}$ microelectrode $\left(r_{\mathrm{T}}=12.5 \mu \mathrm{m}, \mathrm{RG}=9-10\right)$, step size $=25 \mu \mathrm{m}$, translation speed $=20 \mu \mathrm{m} / \mathrm{s}, d=5-7 \mu \mathrm{m}$. b) Cyclic voltammetry performed before and after 2.5 $\mathrm{h}$ of biasing a Pt microelectrode at $0.7 \mathrm{~V}$ in the presence of L-DOPA $2 \mathrm{mM}, \mathrm{pH}=6.5$. c) Chronoamperometry of a solution of L-DOPA $2 \mathrm{mM}$ in phosphate buffer $50 \mathrm{mM}, \mathrm{pH}=6.5$. 
For cyclic voltammetry: scan rate $=50 \mathrm{mV} / \mathrm{s}$. For chronoamperometry: 5 cleaning cycles were performed by applying a potential of $0.7 \mathrm{~V}$ for 20 seconds and $-0.8 \mathrm{~V}$ for 10 seconds. The measured currents at the plateau during the working steps $(0.7 \mathrm{~V})$ are nearly stable and reproducible after the cleaning cycles. Experimental conditions: Pt microelectrode $\left(r_{\mathrm{T}}=12.5\right.$ $\mu \mathrm{m}, R G=9-10)$.

In the SECM imaging routines applied typically in this chapter, the microelectrode traveled twice over the same line scan (i.e. forward and backward) before it was perpendicularly displaced to carry out the next line scan. In this way, two different images were recorded in one experiment by changing the experimental parameters between the forward and reverse scans. Herein, during the forward line scans, a potential of $0.7 \mathrm{~V}$ for the oxidation of L-DOPA was applied for SECM imaging, while on the reverse scans the microelectrode was switched to $-0.8 \mathrm{~V}$ for the electrochemical cleaning of the microelectrode and at the same time to detect the tyrosinase location using the oxygen reduction method. Before each forward line scan, a delay period of 2 $\mathrm{s}$ was introduced to achieve the steady state conditions at the microelectrode.
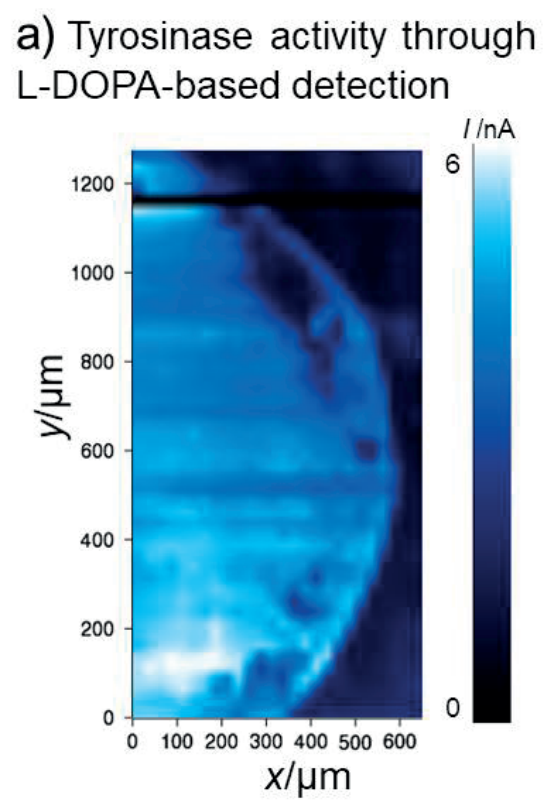

b) Tyrosinase location through the oxygen reduction method

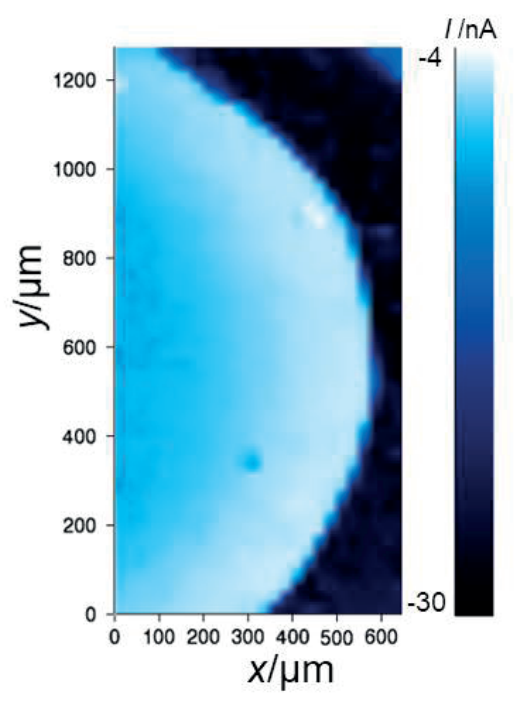

Fig.3.6 SECM images of a tyrosinase spot adsorbed on a PVDF membrane in $50 \mathrm{mM}$ phosphate buffer by monitoring (a) the tyrosinase activity in presence of L-DOPA $2 \mathrm{mM}$, and (b) conforming the tyrosinase location by the oxygen reduction strategy. For (a) forward scan $E_{\mathrm{T}}=$ $0.7 \mathrm{~V}$, for (b) backward scan $E_{\mathrm{T}}=-0.8 \mathrm{~V}$. Other experimental conditions: Pt microelectrode $\left(r_{\mathrm{T}}\right.$ $=12.5 \mu \mathrm{m}, R G=9-10), d=7 \mu \mathrm{m}$, step size $=25 \mu \mathrm{m}$, translation speed $=20 \mu \mathrm{m} / \mathrm{s}$. 
The SECM image obtained in the presence of L-DOPA (Fig.3.6a) depicts the tyrosinase spot as a region of higher current values. Furthermore, the application of the negative potential during the backward scan enables the indirect SECM detection of the adsorbed tyrosinase based on the oxygen detection that is in good agreement with the spatially detected tyrosinase activity (Fig.3.6b), although the enzymatic activity image presents some surface heterogeneity (see Fig.3.6a). The result shown in Fig.3.6a, i.e., an increased current over the tyrosinase is in contradiction from what has been expected. In fact, a decrease on the recorded signal over the tyrosinase active regions due to a depletion of L-DOPA within the gap between the microelectrode and sample was targeted, as the microelectrode and the enzyme should compete for L-DOPA like in the SECM redox competition mode. ${ }^{[170]}$ The result presented in Fig.3.5a suggests that in the critical time scale of the experiment an electroactive species is being generated from the enzymatic reaction between tyrosinase and L-DOPA, and that can be monitored at the scanning microelectrode biased at $0.7 \mathrm{~V}$. Indeed, in the biosynthesis pathway of melanin (Fig.3.7), L-dopaquinone might lead to the formation of hydroxylated aromatic compounds that can be oxidized at similar or lower potentials than L-DOPA. ${ }^{[171]}$<smiles>C#CCCCCCCCCCCCCCCCCC(N)Cc1ccc(O)cc1</smiles>

Tyrosine

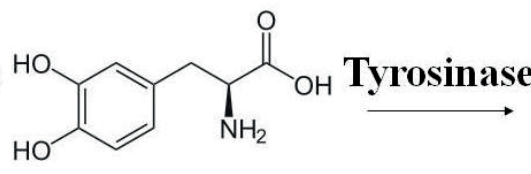

L-DOPA<smiles>NC(CC1=CC(=O)C(=O)C=C1)C(=O)O</smiles>

Dopaquinone

\section{Melanin}

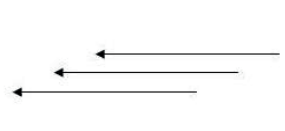<smiles>O=C1C=C2CC(C(=O)[O-])NC2=CC1=O</smiles>

Dopachrome

5,6-Dihydroxyindole (DHI)

Fig.3.7 Abbreviated biosynthesis pathway of melanin: starting from tyrosine and L-DOPA in the presence of tyrosinase.

Similar results were obtained when 4-methyl catechol was employed as enzymatic substrate instead of L-DOPA (Fig.3.8). The underlying mechanisms of the detection of the enzymatic activity of tyrosinase in the performed SECM experiments was further investigated by using differential pulse voltammetry (DPV) and mass spectrometry (MS) experiments (vide infra). 


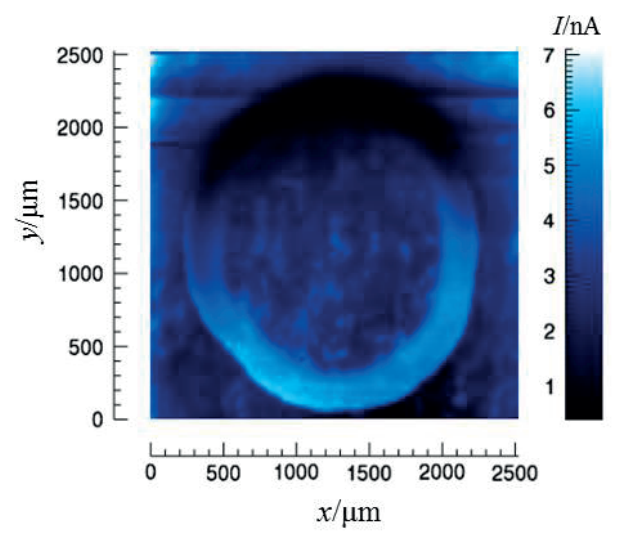

Fig.3.8 Constant height SECM image of an immobilized tyrosinase spot over a PVDF membrane in $2 \mathrm{mM}$ of 4-methyl catechol and $50 \mathrm{mM}$ phosphate buffer, $\mathrm{pH}$ 6.0. Experimental conditions: Forward scan $E_{\mathrm{T}}=0.7 \mathrm{~V}$, reverse scan $E_{\mathrm{T}}=-0.8 \mathrm{~V}$, Pt microelectrode $\left(r_{\mathrm{T}}=12.5\right.$ $\mu \mathrm{m}, \mathrm{RG}=9-10)$, step size $=25 \mu \mathrm{m}$, translation speed $=20 \mu \mathrm{m} / \mathrm{s}, d=7 \mu \mathrm{m}$.

\subsubsection{Mass spectrometry analysis of the enzymatic reaction}

Fig.3.9a displays the mass spectrum of a $2 \mathrm{mM}$ L-DOPA solution where a clear intense signal corresponding to the parent peak of protonated L-DOPA is observed (i.e. $\left.[\mathrm{M}+\mathrm{H}]^{+}=198.19\right)$. This result was obtained by analyzing a droplet of a $2 \mathrm{mM}$ L-DOPA solution over a tyrosinase-free PVDF membrane by using the electrostatic spray ionization (ESTASI) MS. ${ }^{[171]}$ In contrast, when the droplet of L-DOPA solution was placed over a tyrosinase-bound PVDF region, the peak of L-DOPA disappeared rapidly after only $8 \mathrm{~min}$ due to the enzymatic reaction between L-DOPA and tyrosinase (Fig.3.9b). 

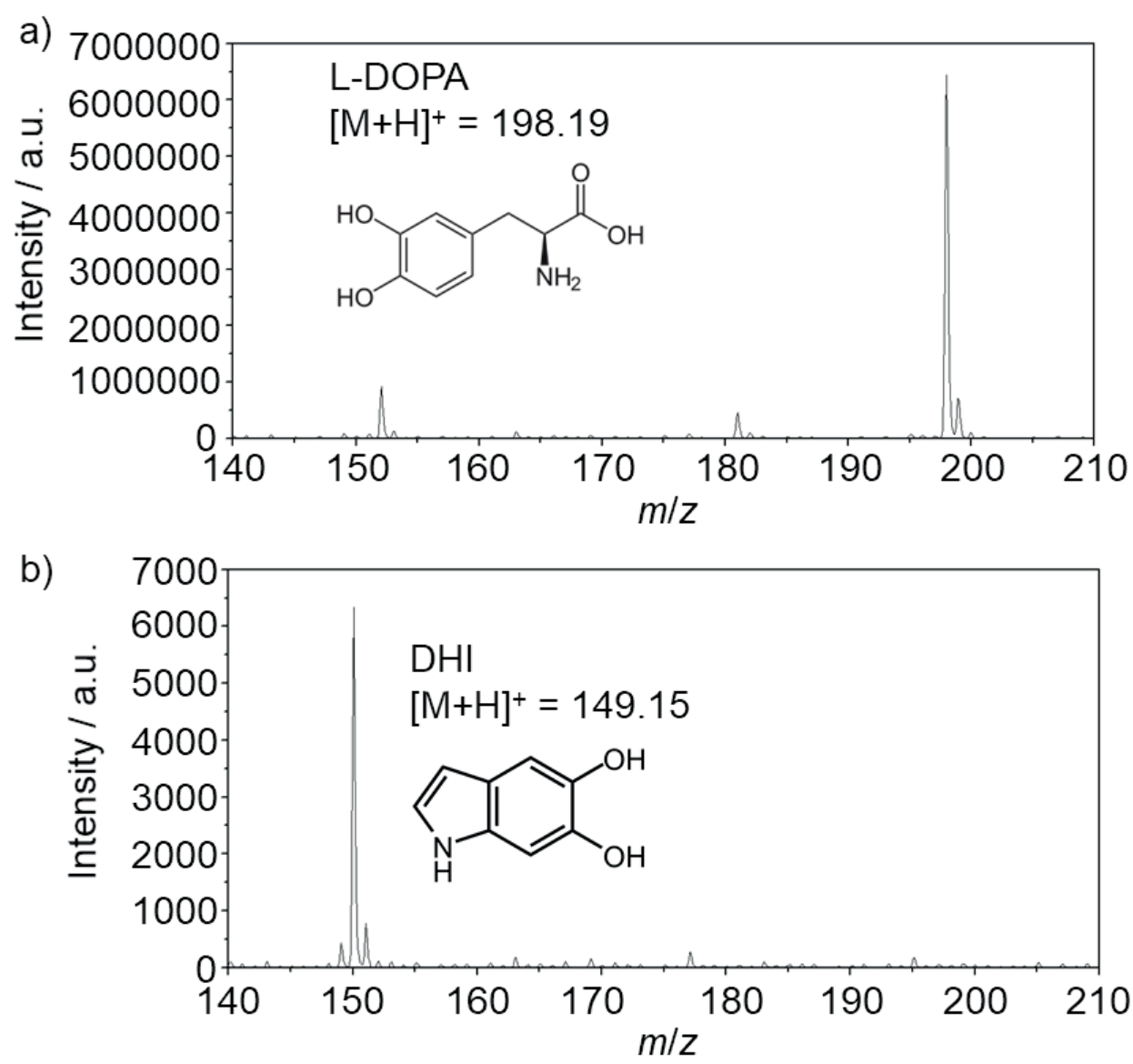

Fig.3.9 a) Mass spectrum of L-DOPA. (b) Mass spectra of L-DOPA (2 mM phosphate buffer, $\mathrm{pH}$ 6) after 8 min reaction with tyrosinase adsorbed on a PVDF membrane.

Indeed, a new peak located at $150.15 \mathrm{~m} / \mathrm{z}$ appeared as a result of the enzymatic reaction. The peak corresponds to 5,6-dihydroxyindole (DHI). As reported previously, DHI has been recognized as one of the products of the reaction between L-DOPA and tyrosinase. ${ }^{[171]} \mathrm{CV}$ and DPV experiments of DHI solutions showed oxidation processes taken place at potentials close to the one of L-DOPA (Fig.3.10). 
a)

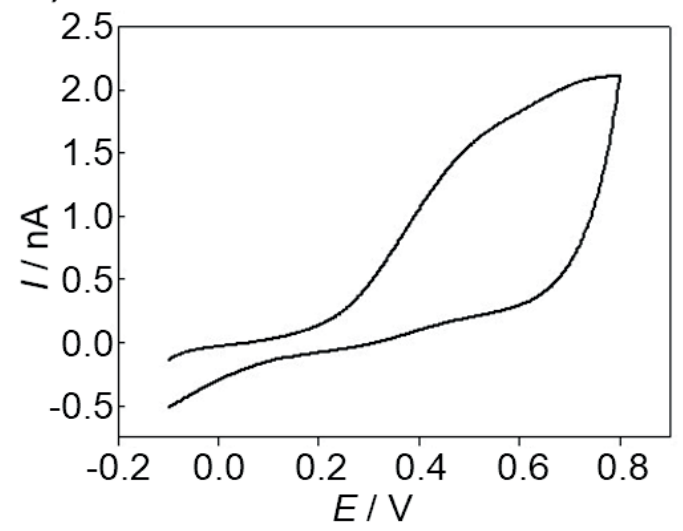

b)

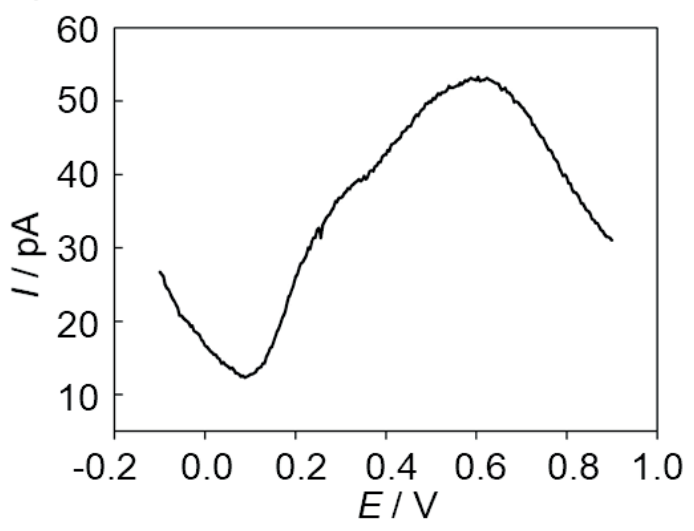

Fig.3.10 a) Cyclic voltammetry and b) DPV of a solution of $1 \mathrm{mM}$ DHI at a Pt microelectrode $\left(r_{\mathrm{T}}=12.5 \mu \mathrm{m}, R G=9-10\right)$. For $\mathrm{CV}$ : scan rate $=50 \mathrm{mV} / \mathrm{s}$. For DPV: $E_{\text {step }}=4 \mathrm{mV}$, pulse amplitude $=10 \mathrm{mV}$, pulse time $=0.2 \mathrm{~s}$, scan rate $=4 \mathrm{mV} / \mathrm{s}$.

As a result, it can be concluded that the SECM visualization of the tyrosinase enzymatic activity is due to the detection of DHI (and perhaps other electroactive species) as a product of the enzymatic reaction between tyrosinase and L-DOPA (Fig.3.11). Hence, the developed tyrosinase detection with L-DOPA is based on the sample generation-tip collection mode.

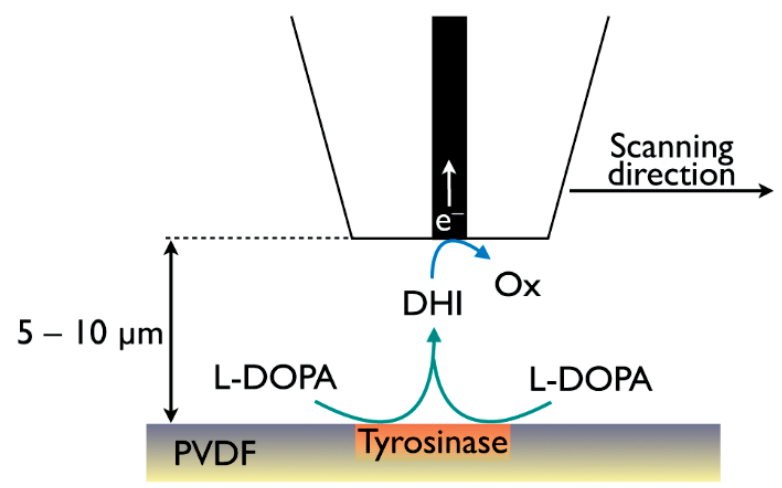

Fig.3.11 Proposed principle of SECM detection of the enzymatic activity of adsorbed tyrosinase spots on PVDF membrane. "Ox" indicates the oxidized products from DHI at the microelectrode.

3.2.4 SECM immunoassay of tyrosinase immobilized on PVDF membrane

In order to verify that the detected enzymatic activity is due to the presence of tyrosinase, an immunoassay strategy has been applied to the tyrosinase spots immobilized on the PVDF membrane. Primary and HRP-modified secondary 
antibodies were used to label the adsorbed tyrosinase and to enable its spatial detection based on the amperometric monitoring of the product $\mathrm{TMB}_{o x}$ of the enzymatic reaction between HRP and $\mathrm{TMB}_{r e d}$ using the SECM sample generation-tip collection mode (Fig.3.12a).

In the SECM image of the tyrosinase spot, the regions depicted with more negative current values correspond to the areas where tyrosinase is present (and the electrochemical reduction of $\mathrm{TMB}_{o x}$ at the microelectrode is possible), in contrast to the less negative current regions where no tyrosinase was present (Fig.3.12b). The inhomogeneous current observed in Fig.3.12b is due to a coffee ring effect during the spot deposition resulting in a higher tyrosinase coverage at the rim of the deposited spot. The main advantage of the immunoassay-based SECM strategy relies on the fact that the tyrosinase detection is based on a specific structural recognition event. As a result, tyrosinase distribution profiles in different samples can be obtained.

a)

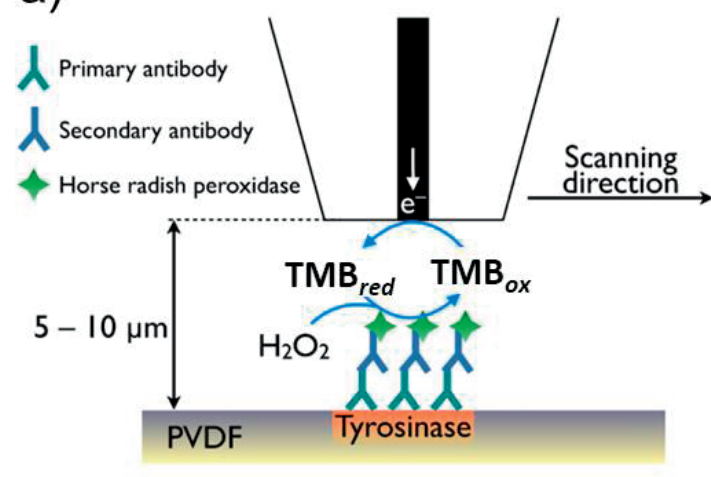

b)

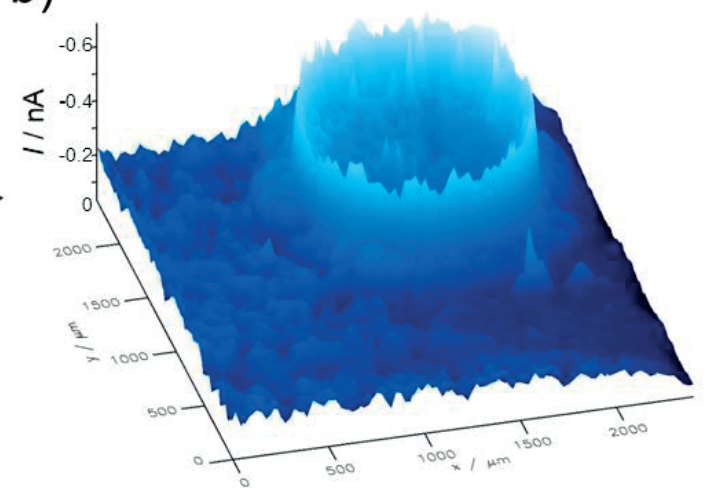

Fig.3.12 a) Schematic representation of the immunoassay of tyrosinase adsorbed on PVDF membrane readout by SECM. b) SECM image of a tyrosinase spot over a PVDF membrane in commercial TMB solution. Experimental conditions: $E_{\mathrm{T}}=-0.15 \mathrm{~V}, \mathrm{Pt}$ microelectrode $\left(r_{\mathrm{T}}=\right.$ $12.5 \mu \mathrm{m}, R G=9-10)$, step size $=25 \mu \mathrm{m}$, translation speed $=20 \mu \mathrm{m} / \mathrm{s}, d=7 \mu \mathrm{m}$.

Fig.3.13 displays the CV plot of the TMB solution in which two oxidation peaks refer to the two-electron transfer reaction of TMB. The TMB oxidation peaks can be observed at potentials around $0.15 \mathrm{~V}$ and $0.35 \mathrm{~V}$ when using the Pt microelectrode, while the reduction takes place at around $0.25 \mathrm{~V}$ and $0.1 \mathrm{~V} .^{[172,173]}$ 


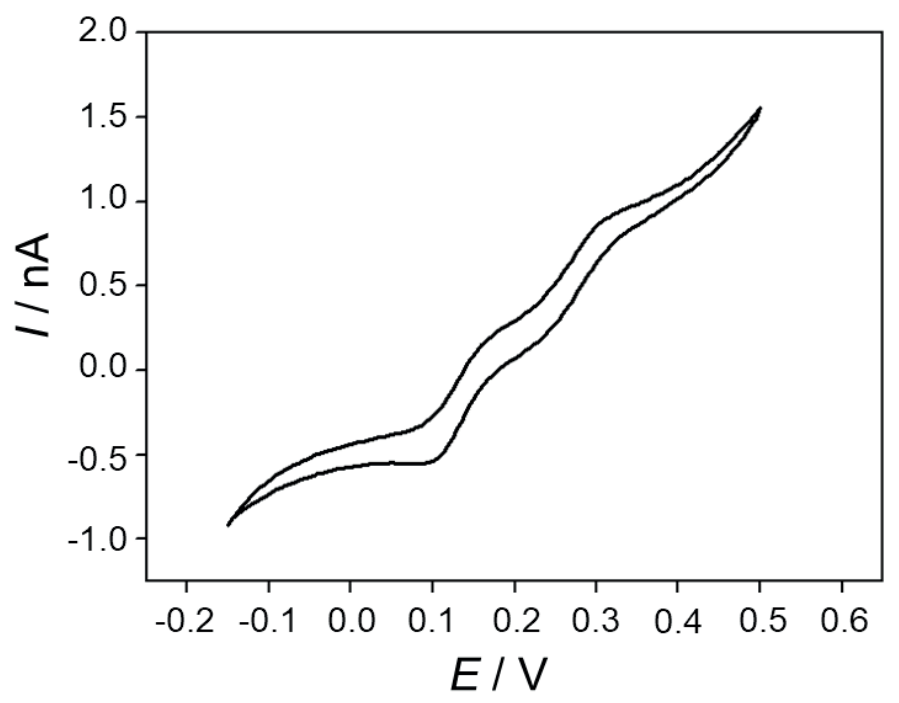

Fig.3.13 Cyclic voltammetry of a solution of $\mathrm{TMB}_{r e d}$ at a Pt microelectrode $\left(r_{\mathrm{T}}=12.5 \mu \mathrm{m}, R G\right.$ =9-10) with a Ag wire as QRE and a Pt wire as counter electrode. Scan rate $=50 \mathrm{mV} / \mathrm{s}$.

\subsubsection{SECM of $\mu$ CP banana on PVDF membrane}

For the analysis of the presence and enzymatic activity of tyrosinase in a $\mu \mathrm{CP}$ banana sample, a tyrosinase spot was deposited next to the printed banana sample as a control pattern. The sample was then examined in the following order: simultaneous application of the L-DOPA-based tyrosinase activity and oxygen reduction methods during forward and reverse scans, respectively, followed by the SECM readout of the tyrosinase immunoassay (Fig.3.14). All SECM images are in good agreement with the obtained optical microscopic image of the studied sample in terms of the shape and position of the tyrosinase spot (Fig.3.14a). However, different features can be recognized on each of the performed SECM experiments of the $\mu \mathrm{CP}$ banana, which can be expected from such complex biological sample. For instance, certain areas depict a low tyrosinase content and activity (between $x=0-500 \mu \mathrm{m}, y=0-200 \mu \mathrm{m}$, Fig.3.14c and $\mathbf{d}$ respectively), but that otherwise in the oxygen method image represent a proteinbound membrane region (Fig.3.14b). The latter can be explained by the fact that not only adsorbed proteins, but also other solid materials probably adsorbed and blocked the PVDF membrane pores. However, all SECM images show the localization and activity of tyrosinase over the region between $x=0-500 \mu \mathrm{m}$ and $y=400-600 \mu \mathrm{m}$. 
a)

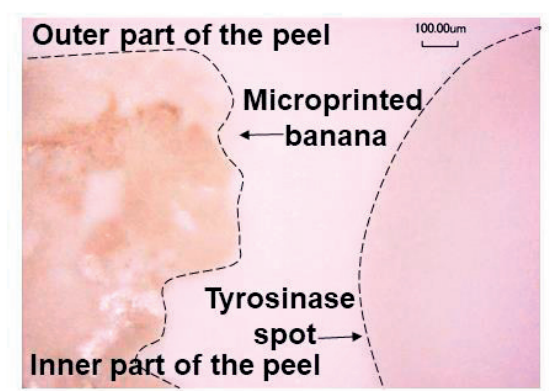

c)

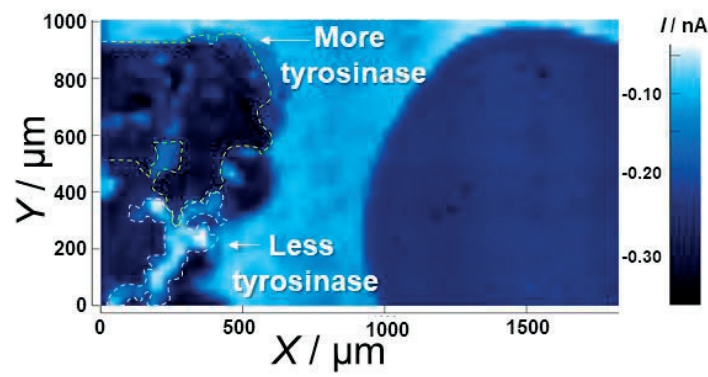

b)
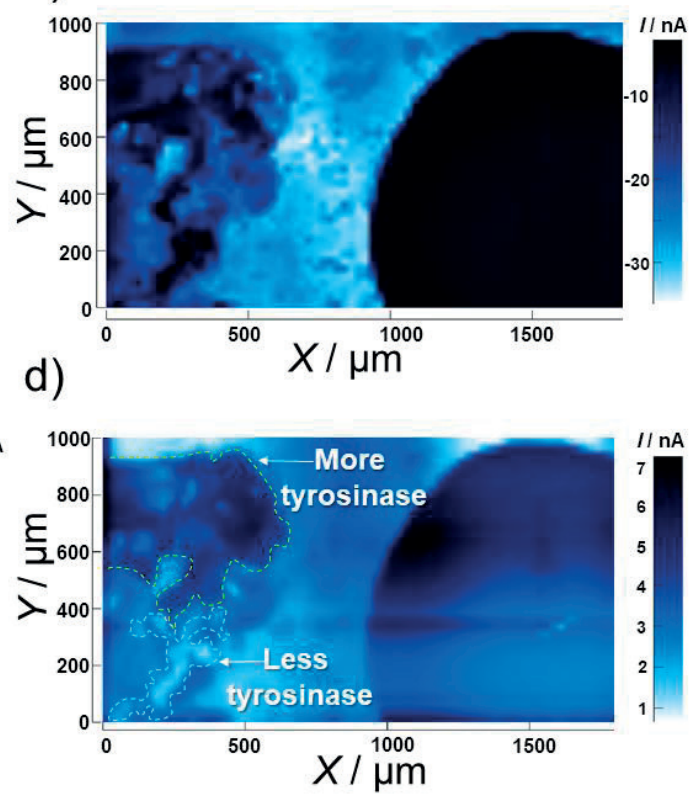

Fig.3.14 a) Optical microscopic and (b-d) constant height SECM images of an adsorbed tyrosinase spot next to a micro-contact printed banana over a PVDF membrane. SECM images were obtained by b) oxygen reduction method, c) immunoassay strategy and d) tyrosinase activity assay. The experimental conditions for (b) and (d) are the same as previously mentioned, while for (c): $\left[\mathrm{TMB}_{r e d}\right.$ commercial solution] $=10 \% \mathrm{v} / \mathrm{v} . E_{\mathrm{T}}=-0.15 \mathrm{~V}$, Pt microelectrode $\left(r_{\mathrm{T}}=\right.$ $12.5 \mu \mathrm{m}, R G=9-10)$, step size $=25 \mu \mathrm{m}$, translation speed $=50 \mu \mathrm{m} / \mathrm{s}, d=7 \mu \mathrm{m}$.

It is important to notice that the distribution of tyrosinase inside the banana peel is not homogeneous and it is mainly located in certain structures of the peel. Interestingly, in Fig.3.14c and d, SECM images display that there is more tyrosinase in the outer part of the peel than in the inner part.

\subsection{Conclusions}

In the present chapter, three different SECM approaches for protein detection on PVDF membranes have been implemented. First, the oxygen reduction method allows for an indirect SECM detection of adsorbed proteins based on the different oxygen concentration inside the PVDF membrane pores, as a consequence of the induced local changes on the membrane hydrophobicity. The main advantage of this concept is that adsorbed protein spots on PVDF membrane can be imaged indirectly without any 
protein labeling procedure or by using redox mediators.

Furthermore, the detection of tyrosinase enzymatic activity was also developed and its principle of detection was examined and confirmed through DPV and MS analysis. Finally, the specific recognition of tyrosinase spots was possible via the SECM readout of an immunoassay strategy. Thus, tyrosinase spots and $\mu \mathrm{CP}$ banana deposited on PVDF membranes were consecutively studied using the three proposed SECM strategies. As a result, a clear and straightforward interpretation of the localization and activity of tyrosinase inside the banana peels was achieved. Besides banana, other biological samples that contain proteins can be $\mu \mathrm{CP}$ on PVDF membranes and similarly analyzed by SECM. It can be envisaged that SECM studies of immobilized tyrosinase on PVDF membrane can be used to understand the influence of different factors on the binding process between tyrosinase and autoimmune antibodies typically generated in Vitiligo patients and which are known to be the responsible for the propagation of this disease. The same strategy presented here can be applied then to study the influence of different parameters, such as temperature, oxygen concentration and presence of tyrosinase inhibitors on the kinetics of fruit ripening. Furthermore, the clear understanding of the results obtained with such complex sample demonstrates that SECM provides all the tools required for performing a detailed characterization of biological surface processes, even by applying consecutive and compatible electrochemical strategies. Thus, SECM can be further employed as a powerful tool for disease diagnostics and molecular screening through electrochemical readout. In the following chapters, more investigation about tyrosinase and melanoma will be presented. 


\section{Monitoring tyrosinase expression in non- metastatic and metastatic melanoma tissues by SECM}

Adapted from: Tzu-En Lin, Alexandra Bondarenko, Andreas Lesch, Horst Pick, Fernando Cortés-Salazar, Hubert H. Girault, Angew. Chem. Int. Ed. 2016, 55, 3813 -3816.

\subsection{Introduction}

Electrochemical methods may represent a promising tool for the early identification of melanoma as they rely exclusively on the electrochemical detection of redox active species related to the presence of biomarkers. Thereby, they can overcome limitations such as optical interferences as seen frequently with conventional histochemistryrelated methods. SECM as a surface reactivity mapping tool with high spatial resolution and sensitivity has been used widely for studying living cell cultures and tissues, although only rarely applied for the latter. ${ }^{[54,174-176]}$ For instance, the enzymatic activity and oxygen production/consumption in plant tissues and micro-tissues have been monitored. ${ }^{[17,18,177-179]}$ SECM has also been applied to study the molecular transport through skin samples. ${ }^{[157,180]}$ The lateral dimensions of tissue samples can approach square centimeters and height differences can be in the critical domain of the SECM probe. Since the probe-sample interaction depends on the working distance $d$, the data interpretation from uneven samples can become cumbersome. A non-controlled $d$ can lead to probe-sample crashes when scanning closely over a tissue leading to irreparable damages in the specimens and contamination of the sensing probe. The former is especially true when using microelectrodes enclosed in a hard-insulating glass body.

In this chapter, a SECM method has been developed and applied for the precise mapping of the tyrosinase distribution in formalin-fixed, paraffin-embedded tissue micro-arrays of skin biopsies taken from nine patients. According to the tyrosinase distribution pattern and its level of over-expression, different melanoma stages as well as normal tissues can be identified. The immunodetection strategy of tyrosinase, developed in the previous chapter (Fig.4.1a), was applied for the monitoring of 
melanoma stages through the electrochemical detection of $\mathrm{TMB}_{\mathrm{ox}}$, which is the enzymatic reaction product between tetramethylbenzidine (TMB), $\mathrm{H}_{2} \mathrm{O}_{2}$ and $\operatorname{HRP}^{[11,136,181]}$ Soft microelectrode probes capable of scanning delicate samples with topographic sample features in a gentle brushing-like contact mode at a constant $d$ were employed (Fig.4.1b) and a conventional Pt microelectrode operated in contactless mode were employed (Fig.4.1c). ${ }^{[71]}$

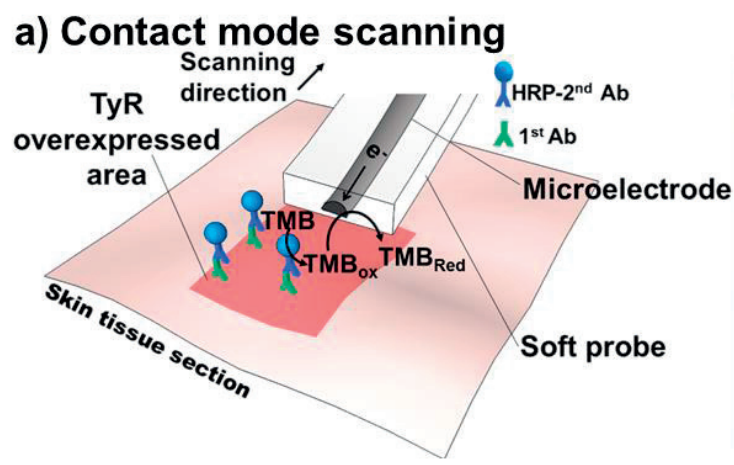

b)

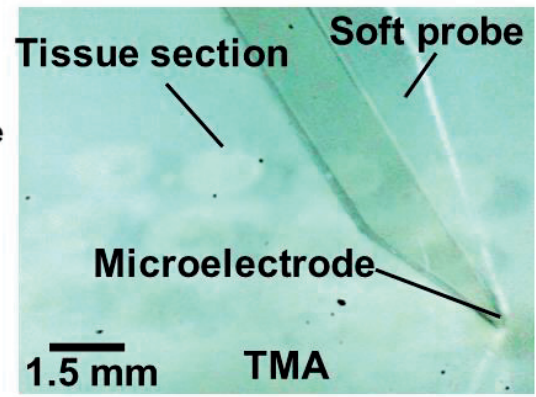

c) Contactless mode

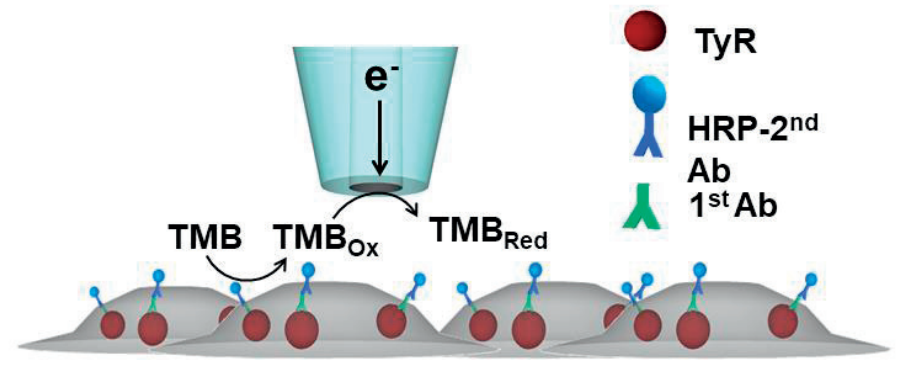

Fig.4.1 a) Schematic representation of the immunoassay based detection strategy to map the tyrosinase (TyR) distribution in tissue sections by using TMB as redox active species and a soft SECM probe. b) Photograph of a soft probe when scanning over a tissue micro-arrays. c) Schematic representation of the applied immunoassay strategy for the SECM sample generation/tip collection mode using the contactless SECM mode with a conventional microelectrode. The strategy is equal to the contact mode apart from the probe employed.

\subsection{Additional experimental details}

Tissue micro-arrays (TMAs) were obtained from Biomax (USA) and used for high-throughput screening of the biomarker tyrosinase among normal skin and different tumor samples obtained from different patients. The tumor samples had been taken out 
from human body by a hollow needle with an inner diameter of about $1.5 \mathrm{~mm}$. As noted by the product supplier, Biomax (USA), the tissue sections were extracted from the patients' skin of arms, feet and chest wall. Different tissues removed from tumor cores were sealed in a paraffin block to form a precisely spaced array pattern. Fig.4.2a displays one tissue micro-array of sectioned skin biopsies of malignant melanoma and normal skin from three different patients and was used for the experiments shown in Fig.4.3a. Another TMA (Fig.4.2b) contained biopsies from another six different patients and was used for the experiments shown in Fig.4.3b-c.

a) TMA 1

N

\section{III}

b) TMA 2

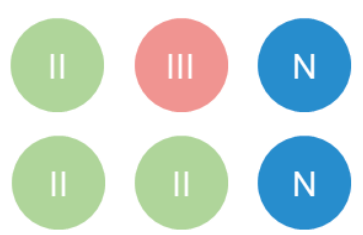

Fig.4.2 a) Tissue micro-array 1 (TMA 1) contained sectioned skin biopsies of malignant melanoma and normal skin from three different patients, and was used in Fig.4.3a. b) Tissue micro-array 2 (TMA 2) contained biopsies from another six different patients and was used in Fig.4.3b. N - normal skin, II melanoma progression stage II, III - melanoma progression stage III. 


\subsection{Results and discussions}

a) Constant height mode
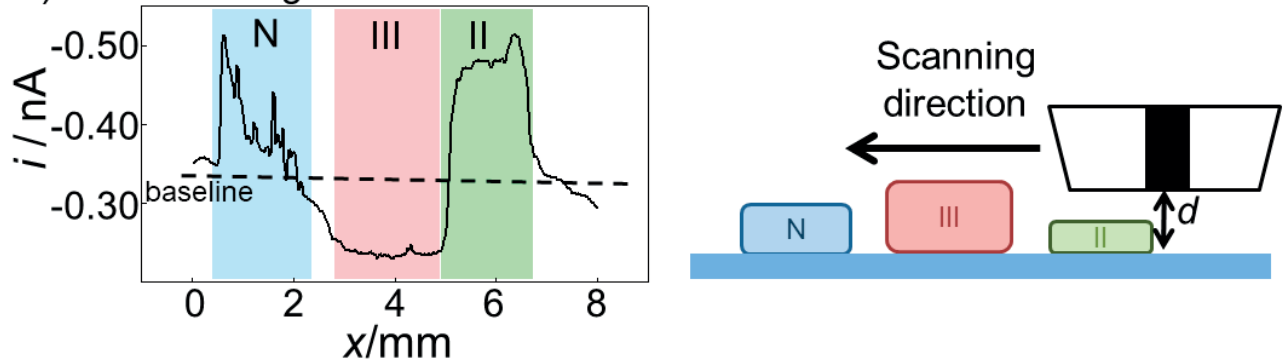

b) Contact mode
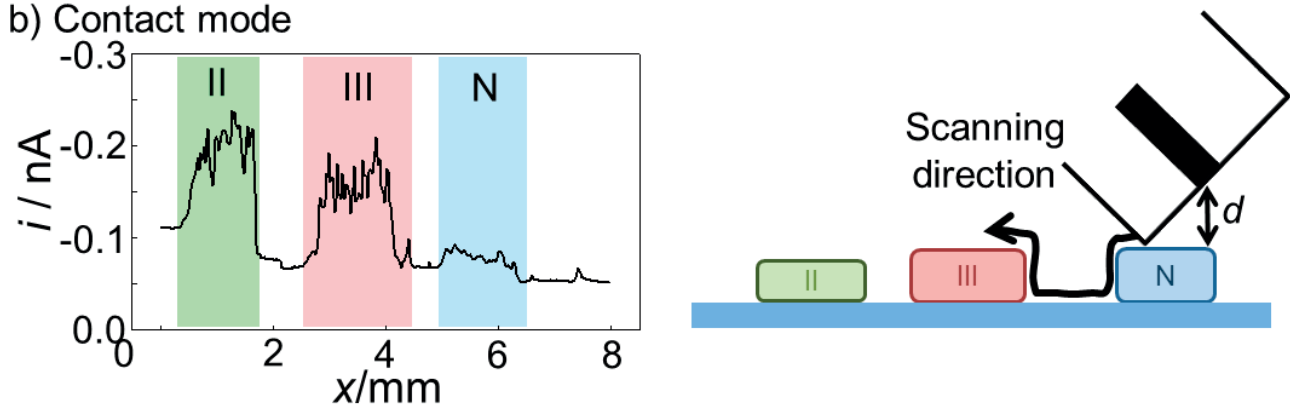

c) Contact mode

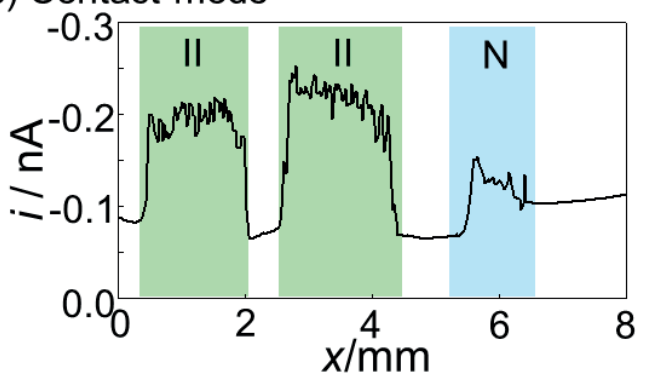

d)

TMA 1

e)

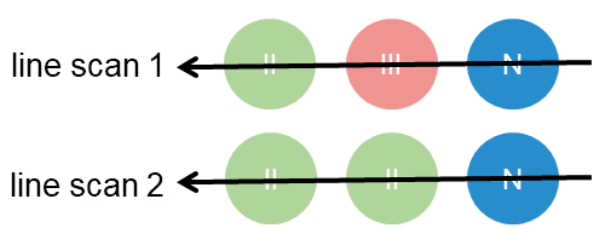

Fig.4.3 a) SECM line scans over TMAs in constant height mode using a conventional microelectrode (a) and in contact mode using a soft probe (b-c). Each tissue section was from a different patient. Left panels show the SECM data, right panels illustrate schematically both scanning modes. Experimental conditions in (a): $\mathrm{WE}=\mathrm{Pt} \mathrm{ME}, r_{\mathrm{T}}=12.5 \mu \mathrm{m}, R G=8-10$, QRE $=\mathrm{Ag}$ wire, $\mathrm{CE}=\mathrm{Pt}$ wire, $E=-0.15 \mathrm{~V}$, translation speed $=20 \mu \mathrm{m} / \mathrm{s}, d=20 \mu \mathrm{m}$ in respect to the microscope glass slide, delay of ADC time $=0.1$ s. $50 \mu \mathrm{M}$ TMB (commercial solution) and 25 $\mu \mathrm{M} \mathrm{H} \mathrm{H}_{2} \mathrm{O}_{2}$ in $10 \mathrm{mM}$ citrate phosphate buffer $(\mathrm{pH}$ ) was used as solution. Experimental conditions in (b-c): $\mathrm{WE}=$ soft carbon microelectrode, $\mathrm{QRE}=\mathrm{Ag}$ wire, $\mathrm{CE}=\mathrm{Pt}$ wire, $E_{\mathrm{T}}=-$ $0.15 \mathrm{~V}$, translation speed $=50 \mu \mathrm{m} / \mathrm{s}, h_{\mathrm{P}}=-50 \mu \mathrm{m}$, delay of $\mathrm{ADC}=0.1 \mathrm{~s} .50 \mu \mathrm{M}$ TMB 
(commercial) and $25 \mu \mathrm{M} \mathrm{H}_{2} \mathrm{O}_{2}$ mixture in $10 \mathrm{mM}$ citrate phosphate buffer (pH 5) was used as solution. Illustration of tissue sections and scanning directions were added in (d) and (e), respectively.

Various TMAs were scanned using a conventional Pt microelectrode and a soft carbon probe. The SECM sample generation-tip collection mode was applied based on the detection of $\mathrm{TMB}_{\mathrm{ox}}$ as produced by the enzymatic reaction of the tyrosinase immunoassay. Fig.4.3 shows SECM line scans over skin biopsy sections from three different patients containing melanoma stages II (non-metastatic) and III (metastatic), and normal skin tissues. A conventional Pt microelectrode in constant height mode was employed by adjusting $d$ to $20 \mu \mathrm{m}$ in respect to the glass support of the TMA, but not to the tissue surface. This working distance is relatively large for SECM, but was required for translating the probe over the tissue sections that were generally higher than $10 \mu \mathrm{m}$ (vide infra). The highest expression level of tyrosinase was observed in stage II tissue samples. A lower level of tyrosinase was found in normal skin where tyrosinase is produced at moderate levels by melanocytes located in the bottom layer of the epidermis. The line scan directions of the contact mode and contactless mode experiments on the TMAs are indicated in Fig.4.3d-e.
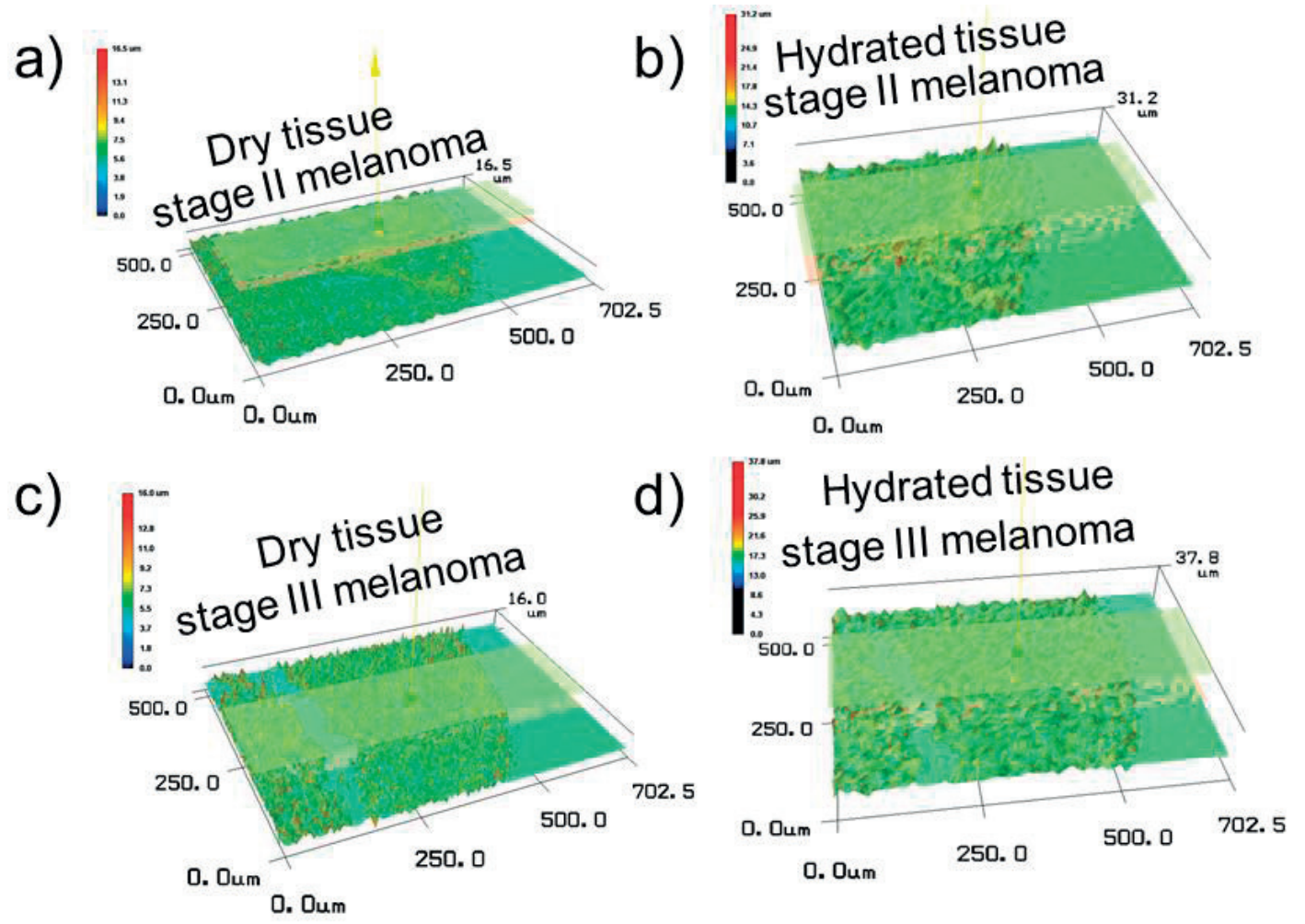
Fig.4.4 Topographic changes of stage II and stage III melanoma skin biopsies before and after hydration. a) Dry tissue section of stage II melanoma. b) Hydrated tissue section of (a). c) Dry tissue section of stage III melanoma. d) Hydrated tissue section of (c).

As it can be seen in Fig.4.3a, over the stage III tissue section much lower currents than the baseline current (i.e. defined as the hindered diffusion current measured over the insulating glass substrate) were detected with the Pt microelectrode. The diffusion of the redox active species towards the microelectrode was significantly blocked due to a very small $d$ over the relatively thick stage III tissue. This observation is supported by topographic measurements of the tissue samples recorded in hydrated state in the experimental solution (Fig.4.4). The average height difference between the dry stage II melanoma tissue and the glass surface was $8.7 \mu \mathrm{m}$ (Fig.4.4a-b). However, after the tissue got hydrated, the average height was $18.3 \mu \mathrm{m}$, demonstrating an increase of the tissue thickness of more than $100 \%$. Similarly, the average height difference between the stage III melanoma dry tissue and glass surface was $10.1 \mu \mathrm{m}$ (Fig.4.4c-d). After hydration, the height was $20.9 \mu \mathrm{m}$, indicating a stronger swelling and thus a more pronounced height. Hence, SECM scanning of tissue sections using the constant height mode faces two major limitations: i) the thickness between tissues can vary significantly and ii) each tissue swells in solution changing its thickness during the measurements.

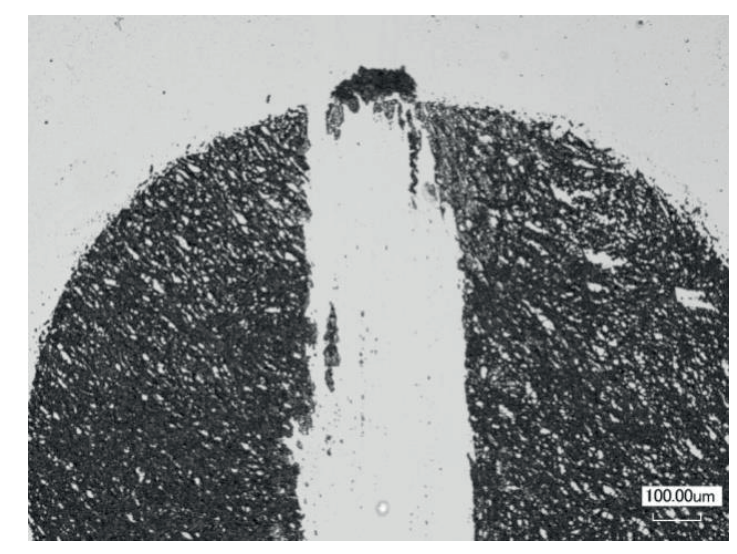

Fig.4.5 Optical micrograph of a micro-scratched tissue section after several repetitions of an SECM line scan using a conventional Pt ME enclosed in an insulating glass sheath. The ME was in contact with the tissue, not with the glass substrate.

It can even be possible that the Pt microelectrode gets into mechanical contact with the tissue inducing micro-scratches or partially detaching tissue material (Fig.4.5). 
Such an event does not only damage the sample, it also contaminates the Pt disk and usually resulting in an electrode cleaning and polishing procedure outside from the electrochemical cell. In order to overcome these obstacles for constant height mode scanning, tissues would have to be immersed in the experimental solution for a prolonged amount of time prior to the SECM experiments until the swelling process is settled. However, different tissue sections will still vary in their heights allowing only an individual investigation in contact-less mode by adjusting $d$ subsequently over each section (Fig.4.6). The experimental time would increase substantially and the stability of the immunoassay as well as the experimental solution and cleanness of $\mathrm{Pt}$ microelectrode surface could hinder long term tissue screening measurements.

a)

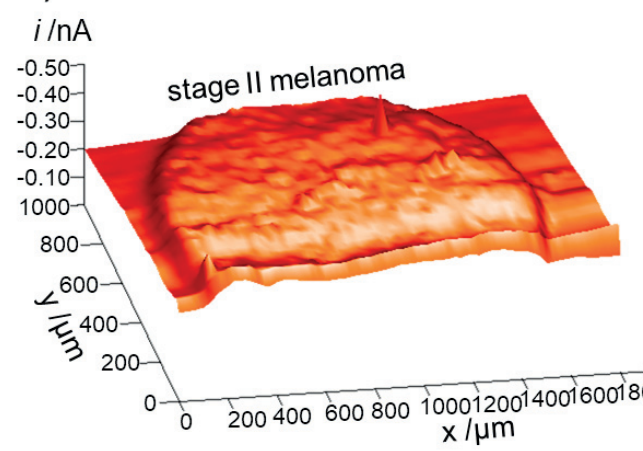

b)

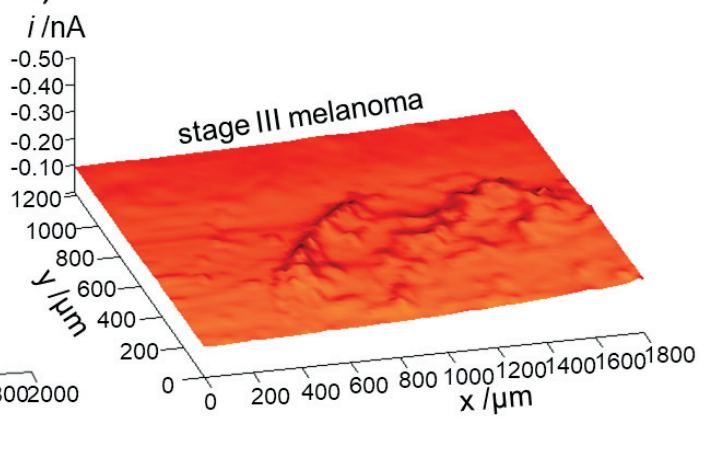

Fig.4.6 Constant height mode SECM image of stage II (a) and stage III (b) melanoma tissues. Experimental conditions: $\mathrm{WE}=\mathrm{Pt}, r_{\mathrm{T}}=12.5 \mu \mathrm{m}, R G=8-10, \mathrm{QRE}=\mathrm{Ag}$ wire, $\mathrm{CE}=\mathrm{Pt}$ wire, $E$ $=-0.15 \mathrm{~V}$, translation speed $=20 \mu \mathrm{m} / \mathrm{s}, d=20 \mu \mathrm{m}$, delay of ADC time $=0.1 \mathrm{~s} .50 \mu \mathrm{M} \mathrm{TMB}$ (commercial) and $25 \mu \mathrm{M} \mathrm{H}_{2} \mathrm{O}_{2}$ mixture in $10 \mathrm{mM}$ citrate phosphate buffer (pH 5) was used as solution.

To overcome these drawbacks, soft stylus probes were employed for the contact mode scanning of biopsies from another six patients (Fig.4.3b-c). In addition, all six tissue sections and the obtained data demonstrate the reproducibility of the SECM approach among samples from different persons. The different tyrosinase levels, i.e. stage II > normal skin, were identified with a high certainty and sensitivity. Stage III melanoma shows a slightly lower tyrosinase content over the entire tissue section than stage II. It has been reported previously that the tyrosinase distribution in stage III melanoma is heterogeneous and in consequence single SECM line scans are not representative. ${ }^{[181]}$ It is worth mentioning that the tissue sections were not damaged 
during the contact mode scanning thanks to the weak forces exerted by the soft probe onto the sample (Fig.4.7). ${ }^{[70-72]}$ The blue color in Fig.4.7 originated from $\mathrm{TMB}_{\mathrm{ox}}$ precipitated on the tissue sections after long term exposure to the assay and allows an optical analysis of the samples. In fact, the location of the precipitation of $\mathrm{TMB}_{\mathrm{ox}}$ showed a strong correlation with the tyrosinase distribution, which is in good agreement with line scan results in Fig.4.3b. However, the original color of the stage III melanoma tissue section was dark brown as a result of the presence of melanin, which can still be seen in the tissue section after TMB exposure (Fig.4.7, middle). Therefore, the color related to $\mathrm{TMB}_{\mathrm{ox}}$ is strongly interfered by the original color of the tissue.

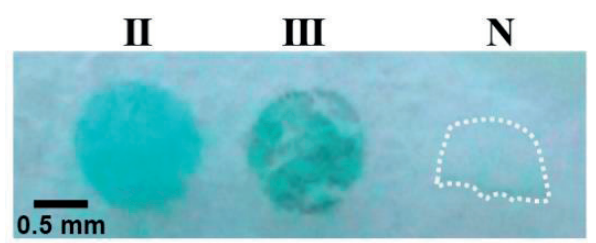

Fig.4.7 Photograph of a TMA containing stage II and stage III melanoma as well as normal skin after the line scan experiments shown in Fig.4.3b. White line as guide to the eye for the normal skin sample.

SECM images of normal skin, melanoma stages II and III sections were recorded and compared with tissues examined by the conventional immunohistochemistry (IHC) method (Fig.4.8). The SECM image in Fig.4.8a clearly indicates the homogeneous over-expression of tyrosinase in stage II and the heterogeneous distribution in stage III melanoma. This is in a good agreement with the results obtained from the contactless SECM image in Fig.4.8. The changes of tyrosinase concentration and distribution pattern in different stages of melanoma may result from complexed mechanisms of tumor progression. Tumors tend to develop different mechanisms of immunosuppression to prevent from being destroyed by the immune system. ${ }^{[182,183]}$ Shedding or mutation of the tumor antigens is one of the strategies that facilitate tumor escape from the immune system, leading to tumor proliferation and metastasis. As a tumor associated antigen, tyrosinase is recognized by the immune system and thus a gradual loss of tyrosinase could be of benefit for tumor escape. As a consequence, the tyrosinase distribution is heterogeneous and slightly decreased in stage III melanoma in accordance to our presented data. In normal skin tissue, the tyrosinase content increased slightly towards the basal epidermis where normal melanocytes are located. This 
polarity of the tyrosinase distribution as observed is in agreement with reports in literature. ${ }^{[181]}$ Because other tyrosinase distribution patterns than the one shown are known to be a sign for different tyrosinase -related dermal diseases the presented SECM approach could provide further screening applications.
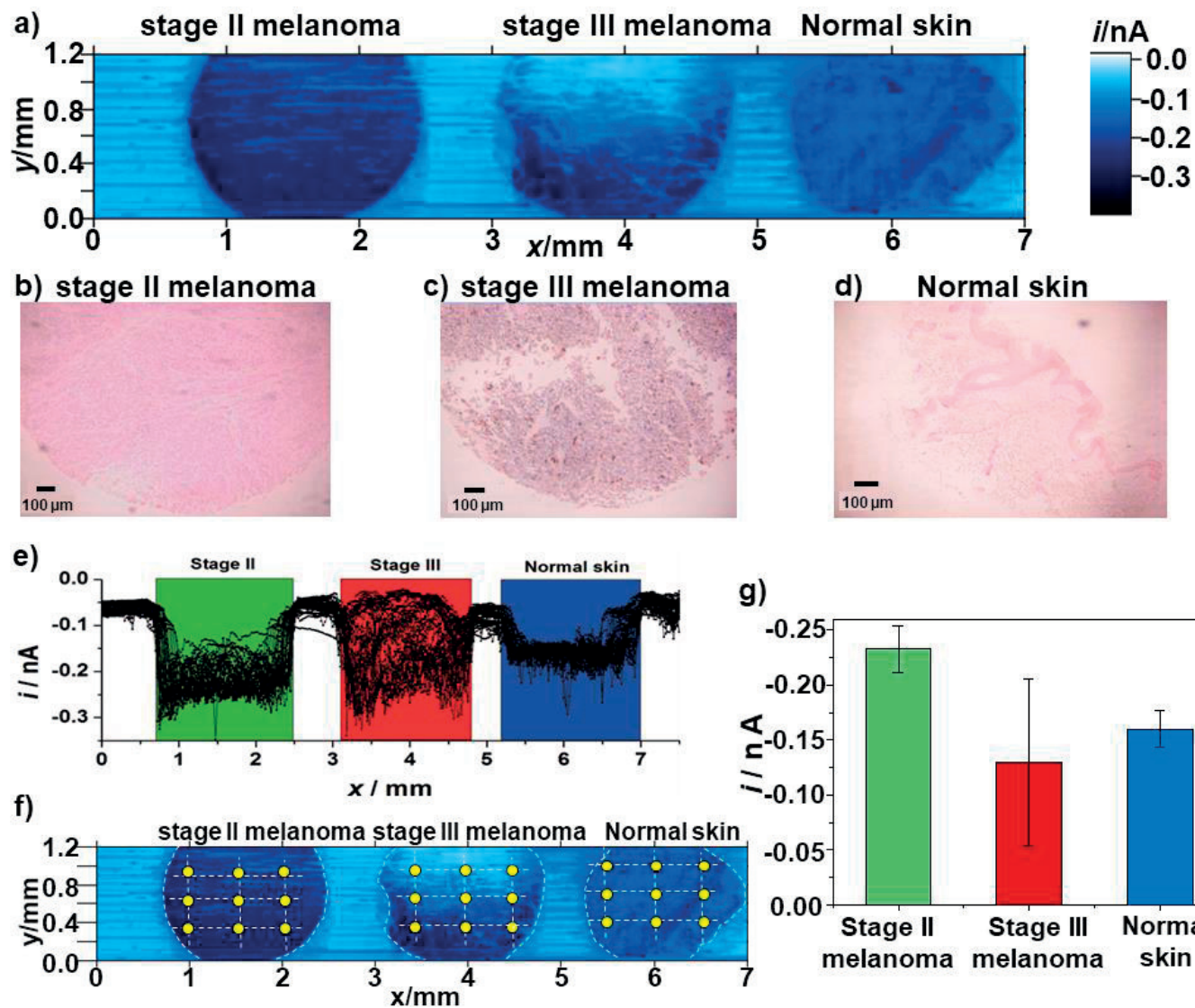

g)

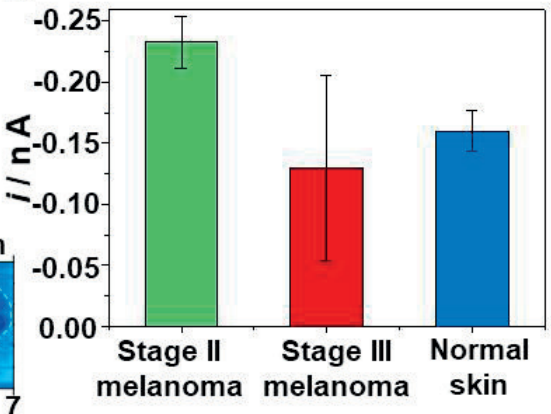

Fig.4.8 a) Contact mode SECM image of stage II and stage III melanoma and normal skin tissues after immuno-staining of tyrosinase. The experimental conditions were the same as in Fig.4.3b. b-d) Optical images of tissues performed by IHC. Similar, but different tissue samples were used in (a) and (b-d), respectively. tyrosinase was stained in pink. e) Average currents of normal skin, stage II and stage III melanomas. Nine current values were extracted and averaged from each tissue section. f) 2D plot of all line scans of the SECM image in Fig.4.8a.

Conventional IHC was performed for comparison with the SECM strategy. In IHC, tyrosinase was labeled with a specific pink chromogen and then observed under an optical microscope. Fig.4.8b shows the homogeneous tyrosinase distribution pattern while Fig.4.8d shows the tyrosinase located along the basal layer of the epidermis. Both figures confirm the reliability of the SECM strategy. However, stage III melanoma in Fig.4.8c contains more melanin and thus its detection is strongly interfered by the dark 
brown color. In contrast, the electrochemical detection of tyrosinase by SECM is not affected by colored background samples (Fig.4.8a).

The importance of mapping the local tyrosinase distribution becomes apparent when averaging the currents from nine representative locations in each tissue section reflecting the global tyrosinase concentration (Fig.4.8f). The currents were extracted every $0.5 \mathrm{~mm}$ in $x$ direction and every $0.4 \mathrm{~mm}$ in $y$ direction. In the column plot in Fig.4.8g, Stage II melanoma shows the highest average current, followed by normal skin and stage III melanoma. Hence, the lower currents of stage III melanoma could be interpreted wrongly as normal skin. However, as can be seen from the SECM image and its 2D plot (Fig.4.8e) the lower average current of stage III is a result of the heterogeneous tyrosinase distribution. Locally, the SECM currents were higher than over stage II. SECM imaging visualizes the abnormal distribution of the tyrosinase (not in the basal layer of epidermis) and confirms the correct identification of stage III melanoma tissue samples. This shows the limitation of traditional sensors that only measure the global concentration of a certain biomarker, while demonstrating the advantage of the spatial resolution. The discrimination between stage II and stage III melanoma is of high relevance because it will drastically influence the medical treatment choice in terms of chemotherapy, immunotherapy or tyrosinase DNA vaccine therapy. ${ }^{[184]}$

The specificity of the tyrosinase immunoassay was confirmed by applying the protocol without primary antibodies (Abs) showing insignificant influence from nonspecific binding. Fig.4.9 shows an SECM image of tissue sections without employing the preparation step of adding primary antibodies but the sample was treated with the same immunoassay in the absence of primary Abs. The recorded current values were significantly smaller and the current over the three tissues showed nearly the same level. 


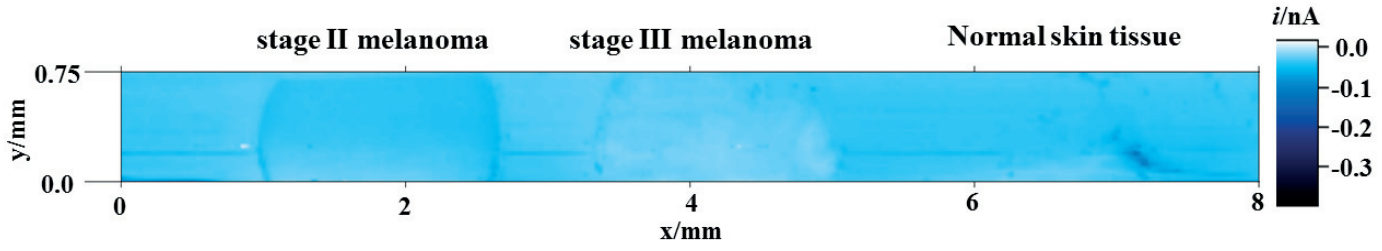

Fig.4.9 SECM image in contact mode of stage II, and stage III melanoma tissue, and normal skin sections without applying the primary antibody. Experimental conditions were the same as in Fig.4.3b.

\subsection{Conclusion}

In conclusion, SECM on skin biopsies from different melanoma patients allowed the accurate monitoring of the tyrosinase expression and distribution in distinct melanoma stages. An immunoassay was employed to distinguish melanoma stages II and III by using soft SECM probes for gentle contact mode brushing of the delicate tissue samples. The transformation from a homogeneous tyrosinase distribution in stage II to a heterogeneous one in stage III was clearly visualized. In comparison with conventional IHC, the SECM concept is not limited by the presence of optically interfering species, such as melanin. Hence, the SECM mapping of tyrosinase might be implemented directly or as complementary prognostic technique for diagnosing metastatic and non-metastatic melanoma stages. This work was the first soft probe SECM approach on thin human tissue sections and was the basis for further developments on the SECM contact mode scanning of various thin and especially thick animal and human tissues for diagnostics in the following chapters. 


\section{From tissues to organs: soft electrochemical probes for scanning human and animal tissues}

\subsection{Introduction}

The visualization of diagnostics biomarkers inside tissue sections plays an important role in medical diagnosis, such as melanoma, Alzheimer disease and rheumatoid arthritis. ${ }^{[185-189]}$ In order to understand the complex disease progression processes, the integration of information obtained from biomarkers on the tissue-level is crucial for the study of biological systems. ${ }^{[79,189]}$ One prevalent strategy for the study of cutaneous diseases, such as skin cancer, is skin biopsy. In this technique, a small piece of the skin is taken out invasively from a suspicious area on the skin possibly related to cancer, sectioned into thin layers $(\sim 5-20 \mu \mathrm{m})$ and deposited onto glass slides for pathologists to perform a microscopic analysis using visual inspection. ${ }^{[190,191]}$ The main reasons of using flat slices from a technical point of view (i.e., resolution) include ensuring precise focusing, avoiding strong scattering from irregular surfaces and enabling enough penetration of light sources throughout the entire tissue. ${ }^{[192]}$ In addition, the skin biopsy also allows performing immunohistochemistry on the sections obtained from different depths of skin. From the data interpretation point of view, it is often important to extract information also in the third dimension and thus inside the thick tissue. Therefore, a statistically relevant number of thin tissue sections (e.g. 5-20 $\mu \mathrm{m}$ thick and separated by several tens of $\mu \mathrm{m}$ ) often needs to be analyzed, which can be followed by a computationally-assisted three-dimensional reconstruction of the digitized data. ${ }^{[193]}$

The analysis of thick tissue sections (i.e., tissues with thickness around 1-3 mm) is very interesting since it allows histological examinations of disease states on relatively intact tissue where the cellular architecture is not damaged. ${ }^{[194]}$ Obtaining information from thick tissues prior to slicing or even before biopsy is attractive, for instance to perform a rapid preliminary study either on the tissue surface. In fact, thick tissue sections possess often similar textures and properties as the original tissue just obtained from the live animal body. Hence, thick tissue sections can represent ideal samples to identify and develop promising measurement techniques that are transferable from the 
sectioned thin tissue slices towards in vivo experiments while keeping the required sensitivity and selectivity. Moreover, ultrathin tissue sectioning process for preparing thin tissue slices may induce some artifacts in histopathology test under optical methods. This may lead to misinterpretations in tissue diagnosis. ${ }^{[195]}$ The study of thick tissue samples may also facilitate the determination of tumor margins in space, even on tissues with irregular topology, as no further tissue sectioning is necessary for analysis. However, thick tissue sections (i.e., tissues with thickness around 1-3 mm) or tissues with rugged surface are difficult to be observed under an optical microscope because the microscopic images can be blurred or out of focus. Thus, developing better strategies for investigating thick tissues is an important issue.

In previous chapters, Soft-Probe-SECM has been introduced for melanoma tissue section scanning. The tissues were swelling but were still relatively flat. Thick tissues (thickness in the range of millimeters instead of micrometers) are generally rougher, more elastic and of irregular shape. Furthermore, thick tissue samples are larger (square centimeters instead of square millimeters), which results in long imaging times causing electrode fouling, sample aging, electrolyte decomposition and electrolyte evaporation. Several strategies were proposed to solve these problems. ${ }^{[196,197]}$ The first one is to increase the translation rate and the step size higher than $25 \mu \mathrm{m}$ of the probe (the latter in case of a stop and go movement) to reduce the imaging time. ${ }^{[46]}$ However, SECM signals rely on diffusion control with a time constant given as $\sim r_{\mathrm{T}}^{2} / D$ where $r_{\mathrm{T}}$ is the radius of the metallic disk of a microelectrode (generally between 5 and $12.5 \mu \mathrm{m}$ ) and $D$ the diffusion coefficient (typically around $10^{-5} \mathrm{~cm}^{2} \cdot \mathrm{s}^{-1}$ ). This is generally fulfilled with slow probe translation rates $\left(\mu \mathrm{m} \cdot \mathrm{s}^{-1}\right)$ but results in imaging times that last longer than $24 \mathrm{~h}$ for square centimeter sized areas. Generally, larger step sizes lower the resolution and interferences from convection become non-negligible under fast scan rates. ${ }^{[42]} \mathrm{A}$ high-speed electrochemical imaging approach, which are principally also applicable to SECM, has recently been reported by Unwin and co-workers. ${ }^{[198-200]}$ A spiral scan pattern was implemented instead of applying the typical raster routine to enhance reliable probe translation rates using piezoelectric positioners. Furthermore, nanoprobes that are characterized by fast mass transport rates and the use of current amplifiers with high bandwidths enabled high resolution scanning of 1000 pixels $/ \mu \mathrm{m}^{2}$ with scan rates above $100 \mu \mathrm{m} / \mathrm{s} .{ }^{[198]}$ Another solution to the electrolyte 
evaporation and electrode fouling is to image sub-regions of a large sample area with intermediate renewal of supporting electrolyte. ${ }^{[201]}$ The third strategy is to use multiple electrodes to image large areas using the slow translation rates but decreasing the imaging time by a factor equal to the number of integrated probes. ${ }^{[71,202,203]}$ This has the advantage that standard SECM instrumentation can be used. The electrode arrays can be made for instance of rigid $\mathrm{Pt}^{[204,205]}$ or soft carbon MEs. ${ }^{[71,72]}$ The Pt MEs were conventional SECM glass encapsulated SECM probes that were placed into curved holders by magnetic forces allowing them to slide in contact mode with substrates of significant topography. However, such strategy is not suitable for biological samples, because the pressures exerted by the probes are too high. In contrast, the soft linear array probes are made in soft and bendable polymeric materials enabling gentle contact mode scanning over delicate samples. Recently, eight individually addressable microelectrodes have been integrated into standard soft probe arrays to reduce experimental times roughly by a factor of eight. Nevertheless, hitherto the individual array microelectrodes were embedded in one polymer sheet, which followed topographical obstacles as a single unit. Therefore, all microelectrodes were affected by major topographic features perpendicular to the probe width and about the size of the microelectrodes.

In this chapter, a soft probe array with eight completely independent, parallel microelectrodes is introduced for large area tissue scanning and the investigation of thick tissues (Fig.5.1a). With eight thin MEs brushing over the surface like a comb or sitting on a sample like a spider, this "Spider Probe" can be applied to scan samples of square centimeter sized areas on reasonable time scales avoiding sample aging and electrode fouling as the standard soft arrays can do, but with a higher resolution to surface roughness adapting to topographic changes up to the millimeter scale (Fig.5.1b). Herein, the successful SECM imaging of $i$ ) certain biomarkers, such as S100 proteins and tyrosinase, adapting immunoassay strategies on thick tumor block sections is presented and discussed while ii) tracking hemoglobin $(\mathrm{Hb})$ in blood vessels in mouse heart section. Fig.5.1c demonstrates the capability of spider probe scanning on a glass substrate with a thickness of $1 \mathrm{~mm}$ which clearly affects the traditional soft linear array (Fig.5.1d). 
a) Spider probe

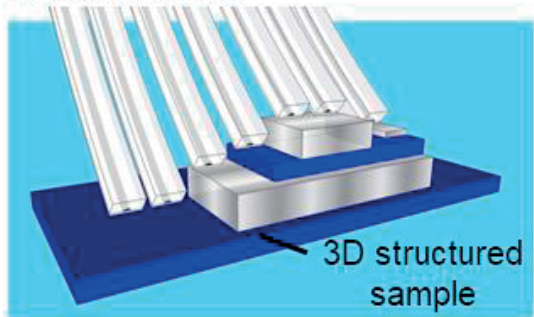

c)

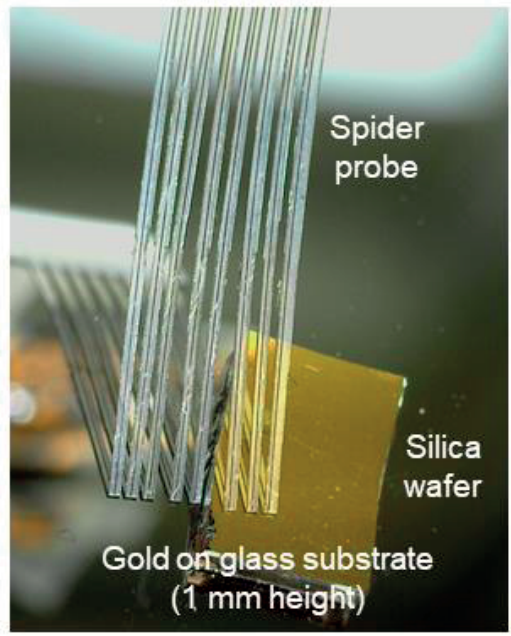

b) Soft linear array

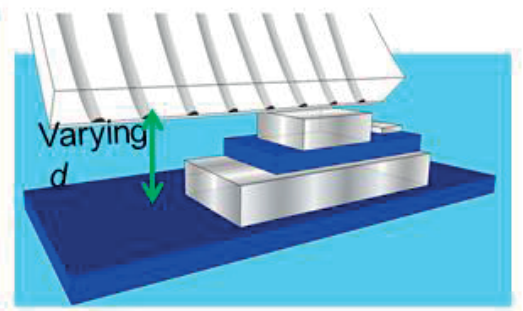

d)

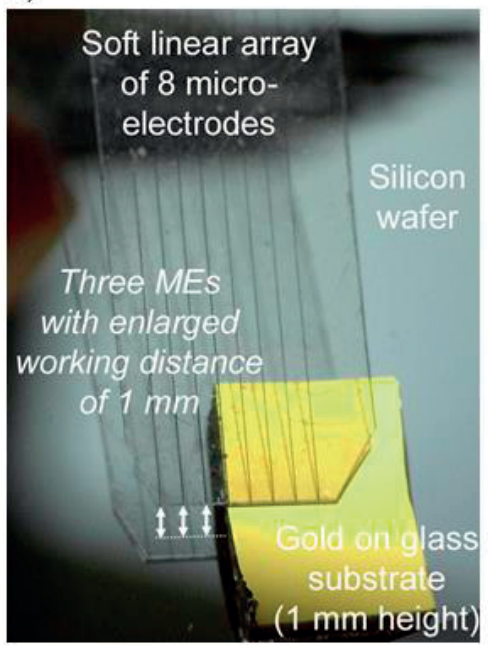

Fig.5.1 a) Schematic representations of the "spider probe" concept (a) and previous design of the soft linear microelectrode array (b) scanning over a 3D sample with significant topographic features. c) Photographs of a" spider probe" array (c) and the conventional soft linear array (d) sliding over a $1 \mathrm{~mm}$ thick Au coated glass slide placed on a Si wafer.

\subsection{Additional experimental details of soft probe fabrication}

All the single microelectrode soft stylus probes used herein were prepared based on the standard procedure introduced previously in Chapter 1. In order to scan thick tissue samples with rough surfaces by Soft-Probe-SECM, the tip of the soft probe was cut with a Spirit 1040-4-SHG laser with $\mu$ FAB Workstation (Spectra Physics Newport) into a stair case shape providing a small probe-sample contact area for high spatial resolution imaging and maintaining the stable scanning properties of the probe when it is pressed against the sample while brushing over it (Fig.5.2). 
a)

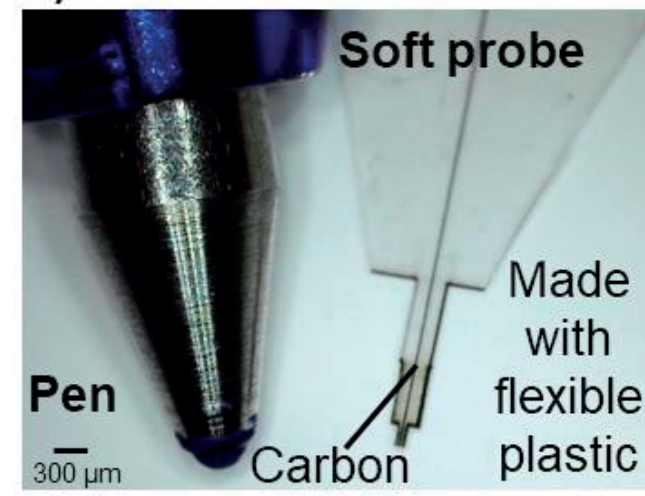

b)

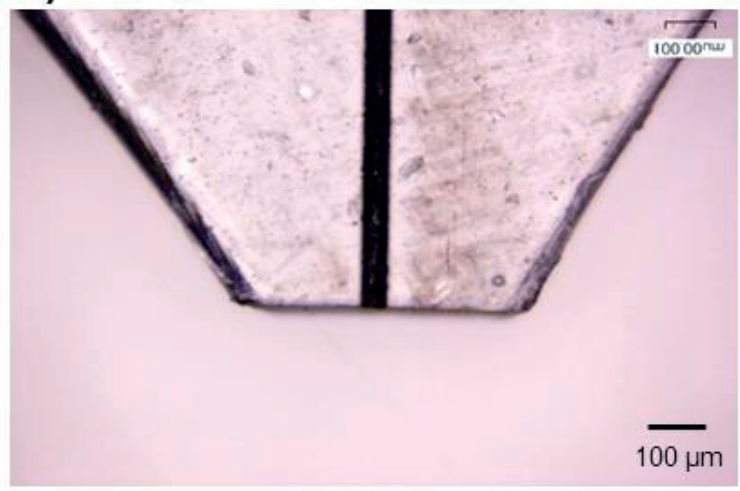

Fig.5.2 Photographs of the soft probe. The width of the probe is around $100 \mu \mathrm{m}$.

All soft microelectrode arrays were fabricated using standard procedure introduced previously in Chapter 1, as well. Briefly, either one or eight microchannels were prepared by UV-photoablation (193 nm ArF excimer laser, OPTEC LSV3) in polyethylene terephthalate (PET, Goodfellow) sheets of $125 \mu \mathrm{m}$ thickness (Fig.5.3). The midpoint distance of the microchannels in an array was $500 \mu \mathrm{m}$ and the width and depth of the channels were $25 \mu \mathrm{m}$ and $15 \mu \mathrm{m}$, respectively. The microchannels were filled with an Electrador carbon ink (Electra Polymer and Chemicals) and after curing at $80^{\circ} \mathrm{C}$ the conductive traces were coated with a Parylene $\mathrm{C}$ layer of $2 \mu \mathrm{m}$ thickness using a Comelec C-30-S Parylene deposition system. The standard linear array of eight microelectrodes was used as a block. The new spider probe was made from such a standard soft linear array by shaping the microelectrodes into "legs" of defined length $(1.5 \mathrm{~cm})$ using a Spirit 1040-4-SHG laser with $\mu$ FAB Workstation (Spectra Physics Newport). The cross-section of all probes and hence the sickle-shaped active microelectrode areas of $(155.2 \pm 18.1) \mu \mathrm{m}^{2}$ size were exposed by razor blade cutting. The width of the individual microelectrode was adjustable between 150 and $300 \mu \mathrm{m}$. Generally, a compromise was found between resolution and scanning stability (vide infra). 
a)

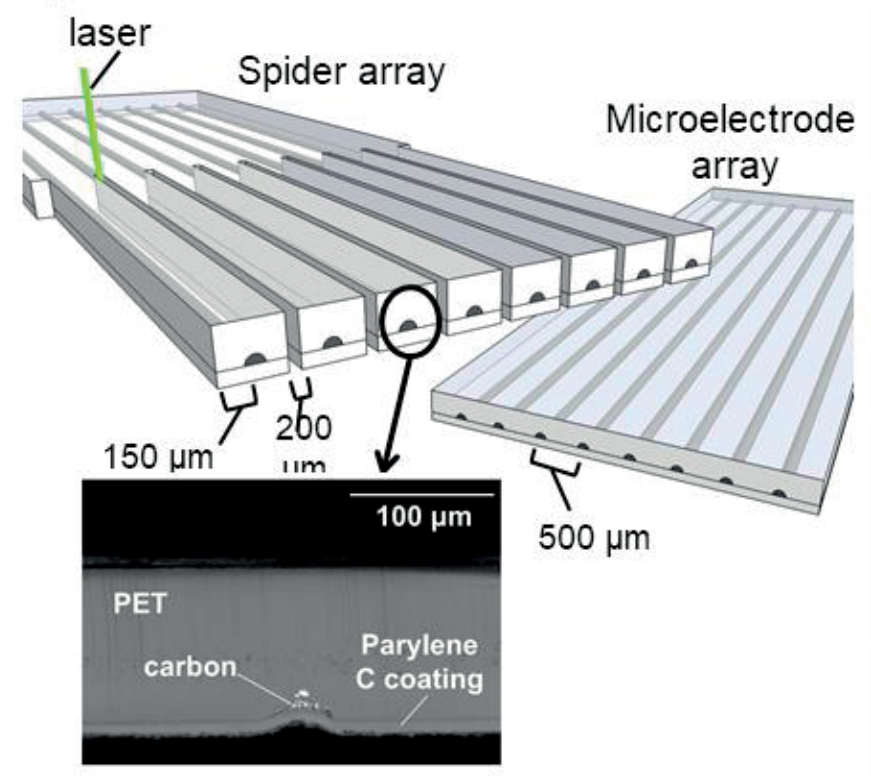

b)

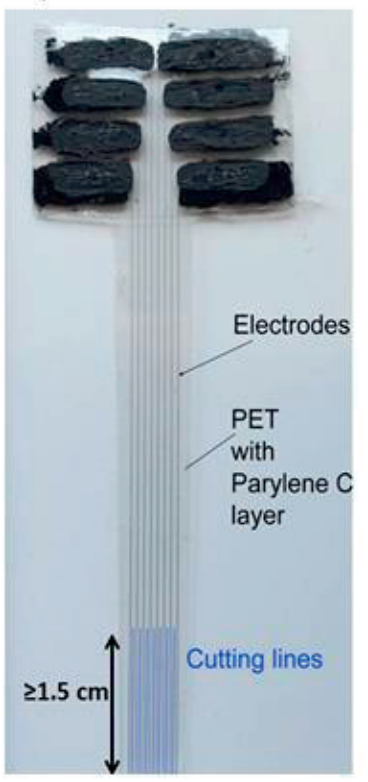

Fig.5.3 Spider probe array fabrication starting from a standard soft linear array probe with eight microelectrodes: a) UV laser ablation to separate all eight microelectrodes equally over a controllable length. The width of the legs and hence the material removed in-between the individual microelectrodes can be set in the laser ablation equipment. b) Photograph of the soft linear array prior to transformation into the spider probe.

\subsection{Results and discussions}

\subsubsection{Spider probe characterization}

The spider probe was electrochemically characterized using first cyclic voltammetry (CV) in bulk solution. In Fig.5.4a, the CVs of the eight microelectrodes showed typical sigmoidal shapes with a diffusion-controlled steady-state current for the electrochemical oxidation of FcMeOH. Slightly different current values were recorded for each microelectrode due to minor variations of the active microelectrode areas as a result of the laser fabrication process, microchannel filling and cutting procedures. The reproducibility of the probe placement and sliding on the substrate was verified by performing feedback mode approach curves on a glass slide and repeating them two times under identical experimental conditions with probe width of $150 \mu \mathrm{m}$ (Fig.5.4bd). The zero position of the $z$-axis is the relative vertical starting point of the probe movement and the positive direction was set down towards the substrate surface. While 
approaching the insulating glass surface the diffusion of the redox mediator got increasingly blocked by the sample leading to a clear decrease in the recorded microelectrode current. At certain points, each individual microelectrode of the spider probe got in mechanical contact with the substrate and started to slide on the glass. The thin spider legs bended slightly differently in solution and possibly had slight various dimensions and shapes resulting both in differences in the absolute working distance and contribution of the diffusion from the solution bulk for each microelectrode. Consequently, the contact point of each microelectrode with the substrate in the overall approach curve plot varied up to $250 \mu \mathrm{m}$ depending on the bending of each probe and how accurately the probe was mounted in the holder. Apparently, this effect can cause lateral offsets between the microelectrodes parallel and perpendicular to the scanning direction during contact mode line scans, which need to be calibrated for SECM imaging. Furthermore, the areas scanned by each microelectrode might need to be enlarged (i.e. larger area for each microelectrode than theoretically required) to guarantee a complete rastering of the entire sample area under study even if two adjacent microelectrodes are spread away from each other (vide infra). Double scanned areas can be easily organized using digital data processing software. Once a microelectrode made contact with the substrate during an approach curve the currents stayed constant as expected for soft probes. In order to level the current responses of the approach curve experiment, a calibration routine was applied that was slightly modified from procedures previously introduced. ${ }^{[41,70,71]}$ From all measured currents $i_{\mathrm{T}, k}$ for each ME $k$ at each measurement point the quantity $i_{\mathrm{T}, \mathrm{off}, k}$ was subtracted $\left(i_{\mathrm{T}, \mathrm{off}, k}\right.$ equals the recorded negative feedback (NF) current $i_{\mathrm{T}, \mathrm{nf}, k}$ of the according microelectrode in contact mode, i.e., $\left.h_{\mathrm{P}}<0 \mu \mathrm{m}\right)$ to give the quantity $i_{\mathrm{T}, k}^{\prime}$, which becomes zero for negative feedback.

$i_{\mathrm{T}, \mathrm{offs}, \mathrm{k}}=i_{\mathrm{T}, \mathrm{nf}, \mathrm{k}}\left(h_{\mathrm{p}}<0\right)$

(Equation 5.1)

$i_{\mathrm{T}, k}^{\prime}=i_{\mathrm{T}, k}-i_{\mathrm{T}, \mathrm{offs}, k}$

(Equation 5.2)

Subsequently, $i^{\prime}$ T, $k$ was normalized by the nf-offset corrected steady-state bulk current $i^{\prime} \mathrm{T}, \infty, k$.

$i_{\mathrm{T}, \infty, k}^{\prime}=i_{\mathrm{T}, \infty, k}-i_{\mathrm{T}, \mathrm{offs}, k}$

(Equation 5.3)

The normalized currents were finally $i_{\mathrm{T}, k}^{\prime} / i_{\mathrm{T}, \infty, k}^{\prime}$

(Equation 5.4) 
a)
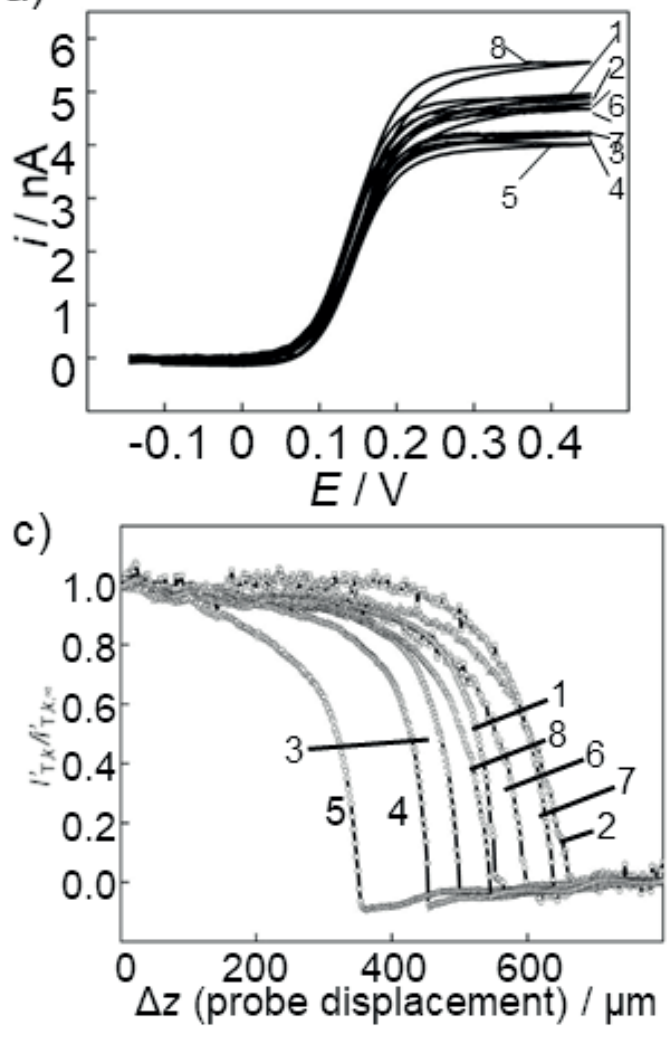
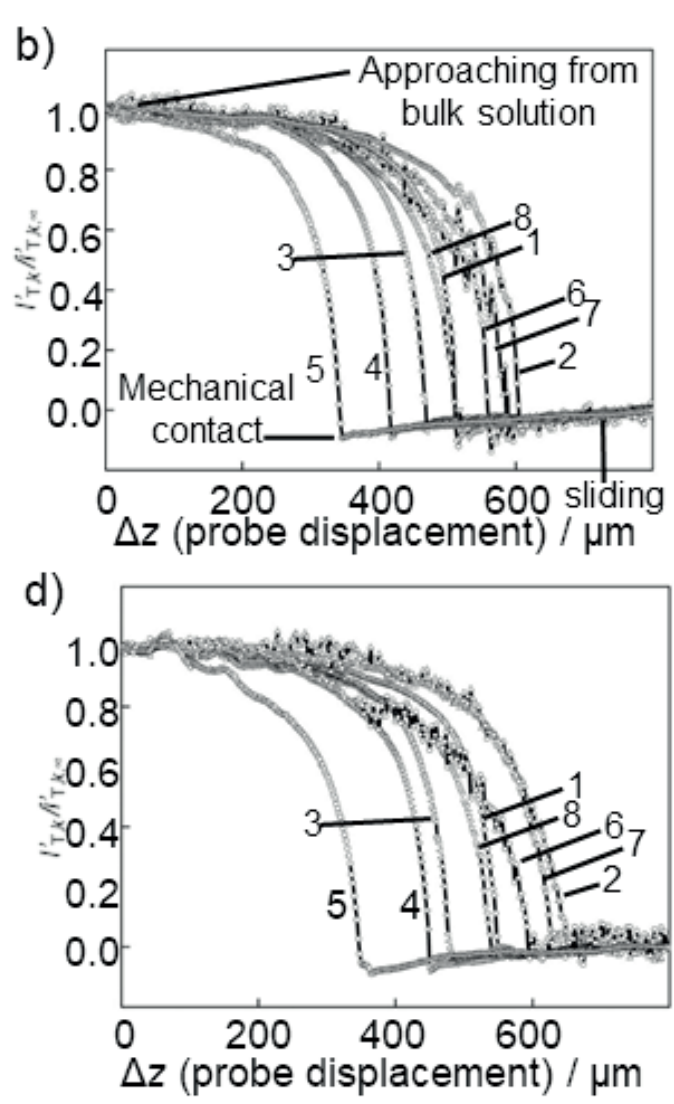

Fig.5.4 a) CVs of the eight microelectrodes within a spider probe in bulk solution. Scan rate 10 $\mathrm{mV} / \mathrm{s}$. b)-d) Three repetitive normalized approach curves of the spider probe over a glass surface. The vertical axis is relative to the start position of the $z$-motor. Experimental conditions: $\mathrm{RE}=\mathrm{Ag}, \mathrm{CE}=\mathrm{Pt}, 2 \mathrm{mM} \mathrm{FcMeOH}$ in phosphate buffer, $\mathrm{pH}$ 6, step size $1 \mu \mathrm{m}$, translation speed $2 \mu \mathrm{m} / \mathrm{s}$. The electrode numbers are indicated in the figures.

The dimensions of the microelectrodes in the spider probe were optimized by using different parameters of probe length (i.e. spider "legs"), thickness of PET and probe width (Fig.5.5c). The optimized length of spider legs, thickness and width was $1.5 \mathrm{~cm}, 125 \mu \mathrm{m}$ and $150 \mu \mathrm{m}$, respectively. Compared with probes made of PET with thickness of $100 \mu \mathrm{m}$, the thicker PET $(125 \mu \mathrm{m})$ provided better stability (less flexible and thus pressing a bit stronger onto the substrate) during operation. The width of the probe can be adjusted according to the sample roughness. However, thinner probes are less affected by sample topography, but less stable during scanning due to pronounced bending. In order to analyze the influence of the probe length, an inkjet printed fully insulating UV curable dielectric cuboid with $35 \mu \mathrm{m}$ thickness was used to analyze the probe scanning characteristics (Fig.5.5a). Fig.5.5b shows the SECM image with a 
spider probe with $0.5 \mathrm{~cm}$ short legs. It can be seen clearly that all legs were perturbed when scanned over the cuboid. At the position of $y=2-4 \mathrm{~mm}$, the microelectrodes lose contact with the substrate. This is due to the bending of one leg caused also a bending of the other seven legs resulting in this specific case in a retraction of various microelectrodes from the substrate surface. Fig.5.5d displays the SECM image of the same cuboid structure using a spider probe with a leg length of $1.5 \mathrm{~cm}$. The recorded currents over the 3D object and PET substrate show constant working distances over almost the entire sample as indicated by similar currents recorded from separate microelectrodes over the insulating PET and printed pattern. However, an edge effect can be identified (at $y=0.8-1.2 \mu \mathrm{m}$ ) where one leg twisted just slightly when being translated over the $35 \mu \mathrm{m}$ high edge increasing and decreasing the working distance. This is indicated by current values that are higher (yellow color) and lower (darker violet), respectively, than the typical negative feedback current on the insulators. Spider probes with other length of legs were also tested. In general, when the spider legs were too short (i.e., $<1.5 \mathrm{~cm}$ ) the main PET probe body transferred the bending motion from one individual leg to all other seven. Hence, the legs were not acting individually. On the contrary, when the legs were too long (i.e., $>2 \mathrm{~cm}$, data not shown) they responded indeed independently to topographic sample features but became very unstable in their scanning performance due to an exaggerated flexibility. 
a)

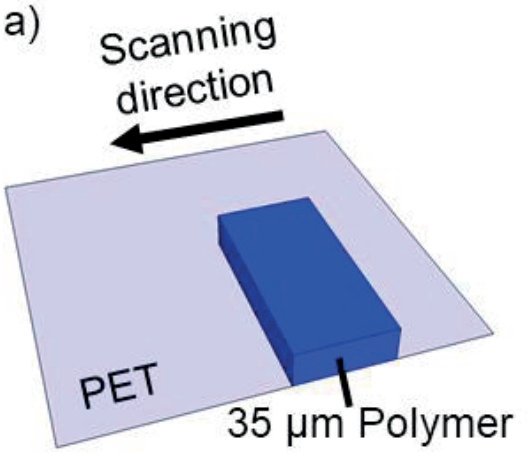

c)

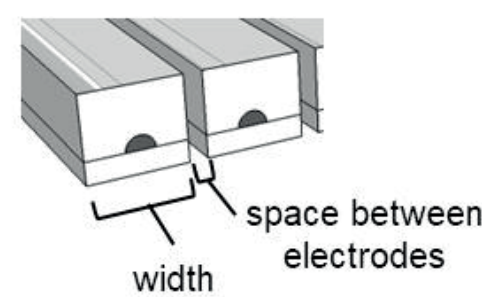

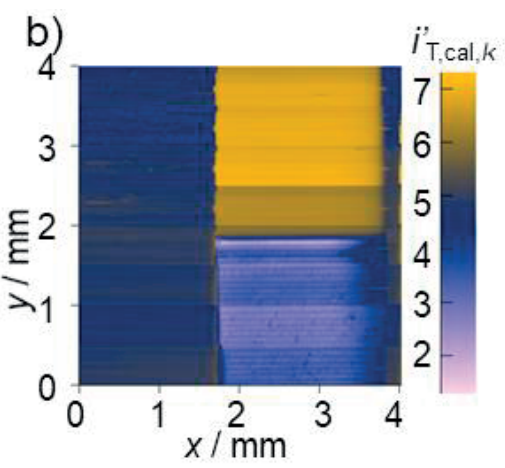

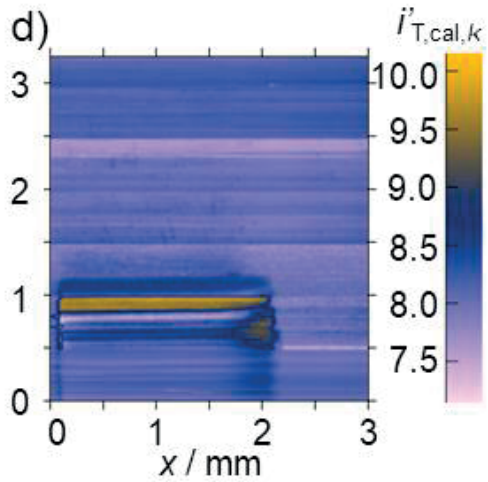

Fig.5.5 a) Schematic representation of the polymer cuboid used for the optimization of probe dimensions. b) Feedback mode SECM image of the 3D sample using an array with $0.5 \mathrm{~cm}$ short spider legs. The 3D obstacle and hence the topographic artifact of $35 \mu \mathrm{m}$ height ranged from $y$ $=0-1.8 \mathrm{~mm}$. c) Schematic representation of two MEs in a soft spider array. d) SECM image scanned with an optimized spider probe with a leg length of $1.5 \mathrm{~cm}$. Experimental conditions: $2 \mathrm{mM} \mathrm{FcMeOH}$ and $10 \mathrm{mM}$ phosphate buffer, $\mathrm{pH}=6.0 . E_{\mathrm{T}}=0.4 \mathrm{~V}, h_{\mathrm{P}}=-50 \mu \mathrm{m}$, step size $=$ $25 \mu \mathrm{m}$, translation speed $=50 \mu \mathrm{m} / \mathrm{s} . i_{\mathrm{T}, \mathrm{cal}, \mathrm{k}}$ is the current after correction. Detail parameters will be displayed in "Additional information for normalization of the SECM images".

The spider probe was tested on a more complex artificial 3D sample made of conductive, inkjet printed carbon nanotube (CNT) patterns and insulation layers with significant topography, i.e., up to $\sim 40 \mu \mathrm{m}$, on flexible and thin polyimide (PI) sheets (Fig.5.6a-b). CNT patterns were covering partially the PI as well as as the insulation layers forming a sample of various heights with different conductivity. The height of the insulation was doubled by printing twice the required number of layers. The CNT coverage and conductivity on the insulation pattern was homogeneous whereas it was heterogeneous on polyimide due to the drying behavior of the printed CNT ink forming islands of higher and lower CNT coverage, respectively. This can be seen by the darker and brighter spots in the CNT patterns. As expected, lower CNT coverage results in 
lower conductivity. The topography of the sample was measured with a mechanical profilometer (Fig.5.6e-f). The directions of the performed scans were chosen perpendicular to the inkjet printing direction and are shown as two black arrows labeled as scan 1 and scan 2 in Fig.5.6b. Due to the nature of inkjet printing and simultaneous UV photo-polymerization curved cross-sections of the patterns were obtained. Two printed insulation layers resulted in a height of $15 \mu \mathrm{m}$ and 4 printed insulation layers were as high as $40 \mu \mathrm{m}$. Please note, that the printing parameters were different for the first and consequently printed layers resulting in a non-linear height increase with the number of printed layers. The base line appears slightly curved due to the flexibility of the thin plastic substrate.

SECM feedback mode imaging was performed on this inkjet printed 3D sample using both the standard soft linear array of microelectrodes and the spider probe (Fig.5.6c-d). Generally, the higher currents resulted from positive feedback were observed on the CNT patterns. The homogeneous CNT coverage on the insulation patterns and the heterogeneous coverage on the PI were clearly revealed in the SECM image. As expected, the linear array as an entire unit was sensitive to the sample topography. The lifting of several microelectrodes off the surface with a significantly increased $d_{k}$ is schematically shown for one exemplary $x$-position (white dashed line in Fig.5.6c). Apart from one microelectrode that was in contact with the sample all other microelectrodes lost the close contact to the substrate causing topography related artifacts in the current signal. In contrast, the microelectrodes of the spider probe acted individually with a constant $d_{k}$ and generated a clear feedback mode image (Fig.5.6d). The measured currents were first calibrated to $i^{\prime}{ }_{\mathrm{T}, k}=0$ and then multiplied by a scale factor $s_{k}$ to set the positive feedback currents to unity. In addition, positional offsets of the individual microelectrodes in $x$ and $y$-direction were adjusted (details see

Additional information for normalization of the SECM images). The calibration of microelectrode variances in SECM feedback mode images on insulating and conducive substrates in Fig.5.6c-d is described in the following:

a) Calibration of the current values

$i^{\prime}{ }^{\prime}$,max,$k$ - current for diffusion controlled redox mediator regeneration $i_{\mathrm{T}, \mathrm{max}, k}$ after applying Equation 5.5:

$i_{\mathrm{T}, \max , k}^{\prime}=i_{\mathrm{T}, \max , k}-i_{\mathrm{T}, \mathrm{offs}, k}$

(Equation 5.4) 
$s_{k}-$ scale factor to set current for diffusion controlled redox mediator regeneration to unity:

$S_{k}=\frac{1}{i_{\mathrm{T}, \max , k}^{\prime}}$

$\frac{i_{\mathrm{T}, k}^{\prime}}{i_{\mathrm{T}, \max , k}^{\prime}}=\left(i_{\mathrm{T}, k}-i_{\mathrm{T}, \mathrm{offs}, k}\right) \cdot s_{k}$

(Equation 5.7)

b) Calibration of positional offsets

$x_{k}^{\prime}=x_{k}-x_{\text {offs, } k}$

$y_{k}^{\prime}=y_{k}-y_{\text {offs, }, k}$

(Equation $5.8 \& 5.9)$

The characterization of spider probe demonstrates its capability and thus can be further used on scanning of biological samples such as thick melanoma tumor sections. 
a)

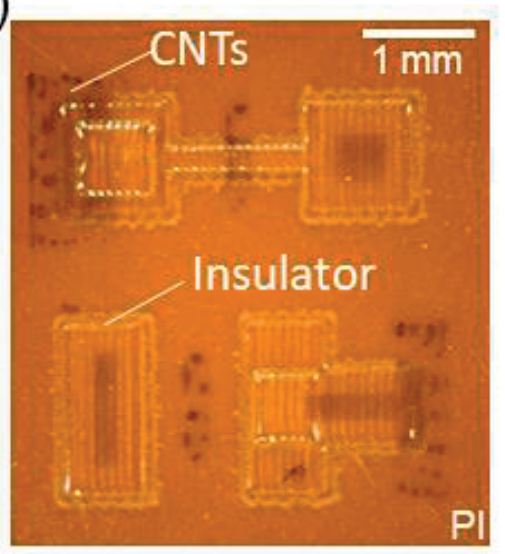

c)

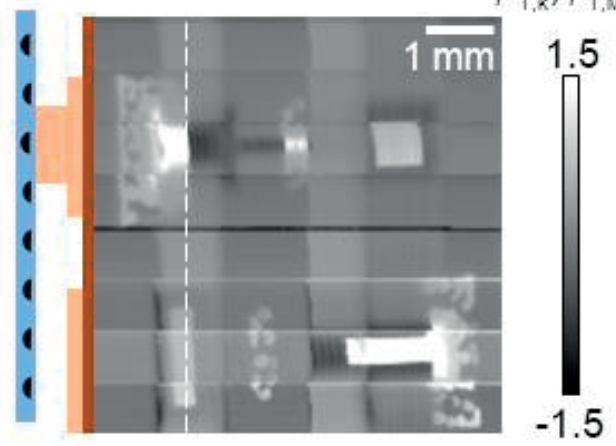

e) Scan 1

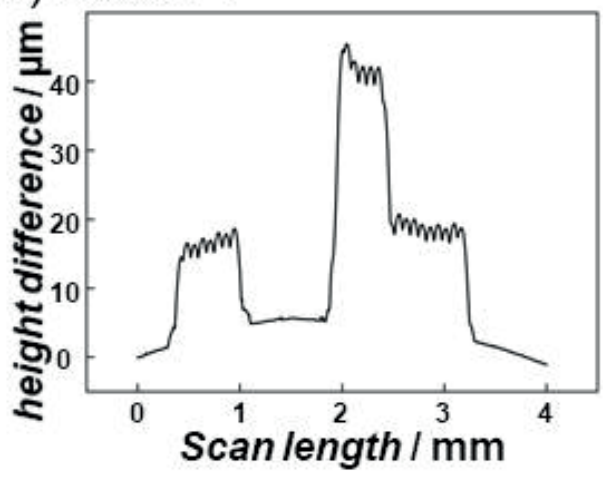

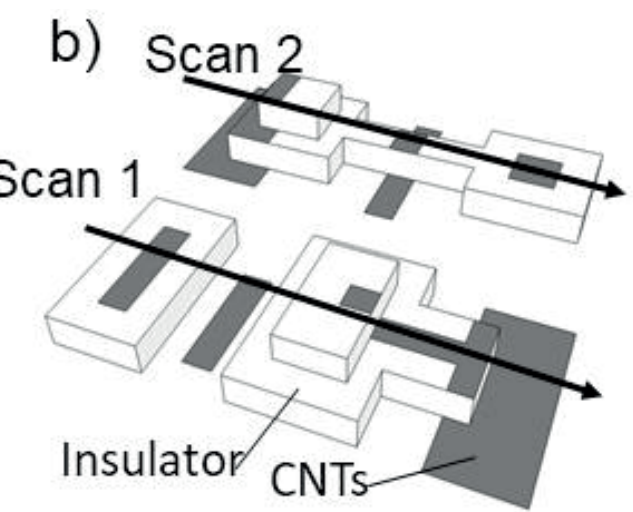

d)

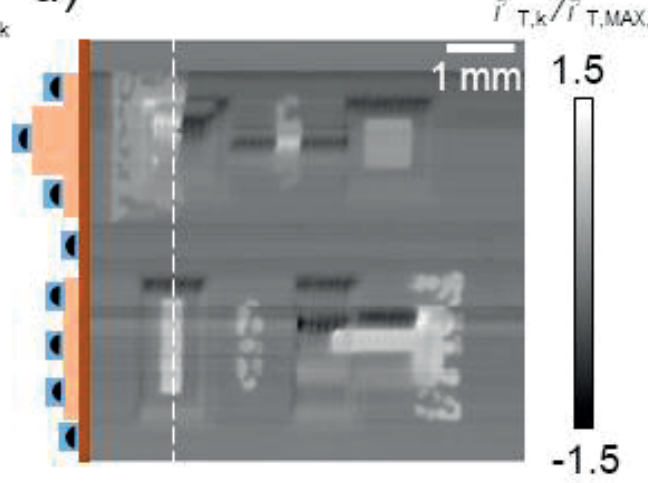

f) Scan 2

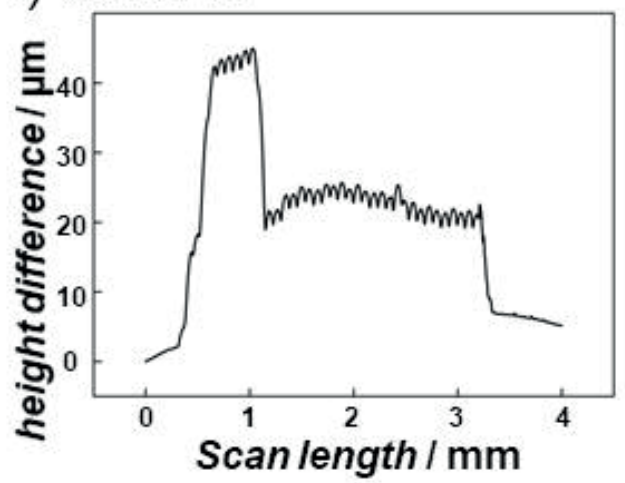

Fig.5.6 a) Microscopic image of the inkjet printed 3D sample containing CNT (dark-grayish) and transparent dielectric patterns on a PI sheet. b) Schematic representation of inkjet printed 3D sample containing conductive CNT (grey) and insulating polymer patterns (white). c) Feedback mode SECM image of the 3D sample using a conventional array. The left panels in (c) are schematic representations of the probes and sample at an exemplary $\mathrm{x}$-coordinate (dashed white lines). Experimental conditions: $2 \mathrm{mM} \mathrm{FcMeOH}$ and $10 \mathrm{mM}$ phosphate buffer, $\mathrm{pH}=6.0 . E_{\mathrm{T}}=0.4 \mathrm{~V}, h_{\mathrm{P}}=-50 \mu \mathrm{m}$, step size $=25 \mu \mathrm{m}$, translation speed $=50 \mu \mathrm{m} / \mathrm{s}$. d) SECM image of the 3D sample scanned by a spider probe. The left panels in (d) are schematic representations of the probes and sample at an exemplary $\mathrm{x}$-coordinate (dashed white lines). Experimental conditions: $2 \mathrm{mM} \mathrm{FcMeOH}, \mathrm{H}_{2} \mathrm{O}_{2} 1 \mathrm{mM}$ and $10 \mathrm{mM}$ phosphate buffer, $\mathrm{pH}$ 6. $h_{\mathrm{P}}$ 
$=-300 \mu \mathrm{m}, E_{\mathrm{T}}=-0.2 \mathrm{~V} v s \mathrm{Ag}-\mathrm{QRE}$, step size $=25 \mu \mathrm{m}$, translation speed $=50 \mu \mathrm{m} / \mathrm{s}$. The topography of this sample was scanned with a mechanical profilometer. e) and f) correspond to the two scan directions shown in (b). Parameters for current calibration is in "Additional information for normalization of the SECM images".

\subsubsection{Soft single probe on thick melanoma tissues}

The concept of Soft-Probe-SECM of thick melanoma tissue scanning was first verified by single soft probe. Two SECM detection strategies were applied. First, as shown in Fig.5.7a, an immunoassay strategy was employed for the specific SECM imaging of certain biomarkers in thick melanoma tissues in contact mode. The primary antibody and the secondary antibody labeled with horse radish peroxidase (HRP) were applied to detect the biomarkers tyrosinase and S100 protein. HRP catalyzes the oxidation of $\mathrm{FcMeOH}$ to $\mathrm{FcMeOH}^{+}$in the presence of $\mathrm{H}_{2} \mathrm{O}_{2} \cdot{ }^{[206]}$ When applying a reduction potential of $-0.2 \mathrm{~V}$, the $\mathrm{FcMeOH}^{+}$produced by HRP can be collected at the microelectrode, causing an increment in current signals over areas with significant biomarker concentration. Therefore, the SECM SG/TC mode was used. Second, as shown in Fig.5.7b, the detection of redox active proteins, which play an important role in biological functions, can be achieved using the SECM feedback mode. Here, hemoglobin ( $\mathrm{Hb}$ ) was detected. Hemoglobin present in blood is an iron containing protein functioning as oxygen transport protein. ${ }^{\text {[207-209] }}$ Hemoglobin has excellent electron transfer ability and thus can be modified on the electrode or used as a catalyst. ${ }^{[210,211]}$ This property allows the regeneration of redox mediators, leading to an increased current over the area when imaging with SECM. 

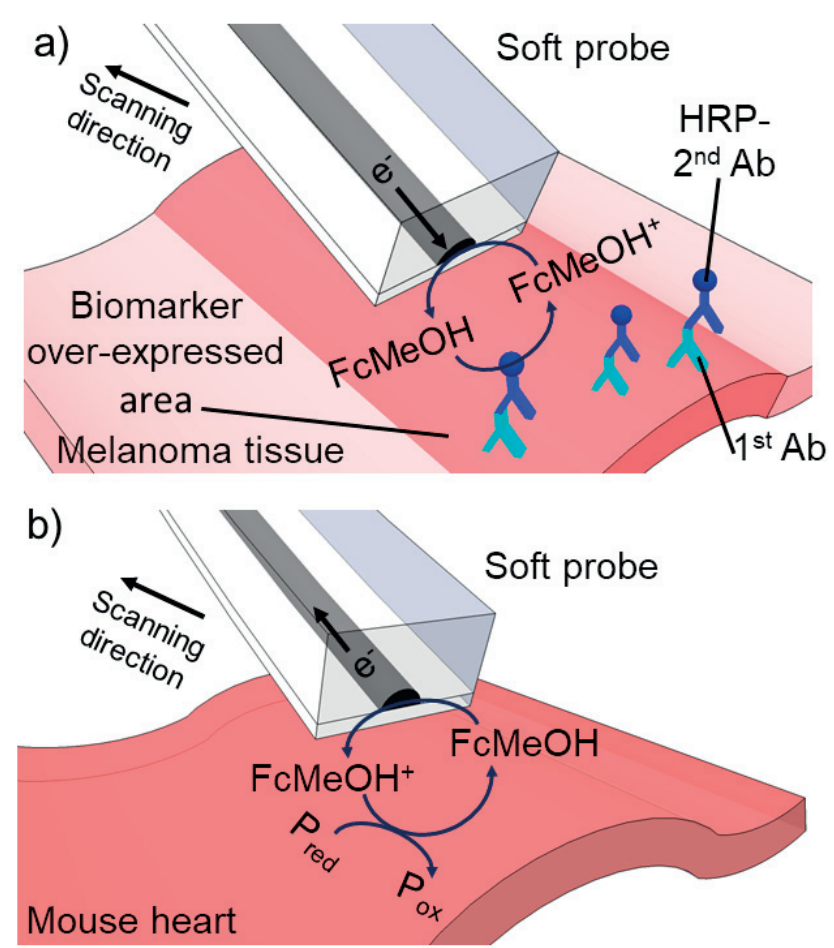

Fig.5.7 a) Schematic representation of scanning the over-expressed area over the melanoma tissue using immunoassay in contact mode. b) Schematic representation of scanning redox active proteins.

The skin biomechanics in thin tissue slides are different than thick tissue sections. The thin tissue slices are attached on glass and supported by the glass but the thick tissue sections are not. Thus, the higher elasticity and lower stiffness in thicker skin tissues may cause higher the uncertainty of topography change of the sample surface. ${ }^{[212]}$ Therefore, scanning thick tissues using conventional SECM is almost impossible.
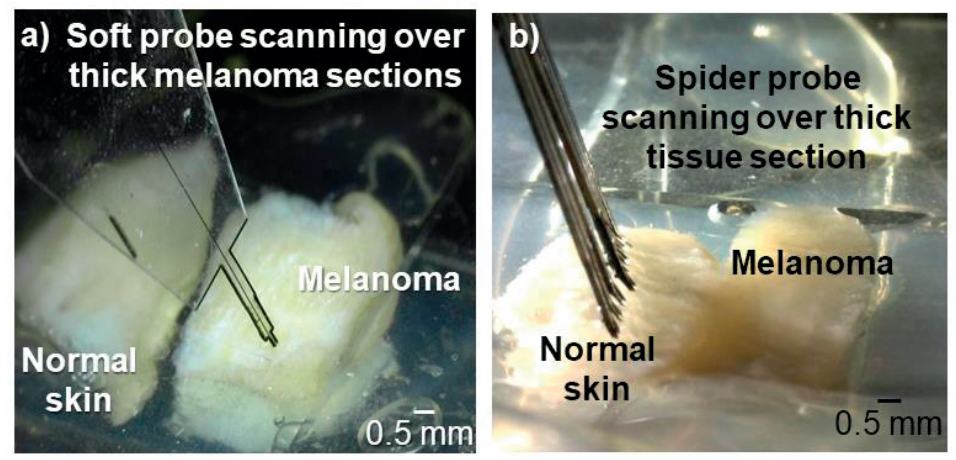

Fig.5.8 Optical pictures of the Soft-Probe-SECM scanning with a) single soft microelectrode and b) spider probe on melanoma and normal skin thick tissue sections after imaging. 
Fig.5.8 displays optical pictures of Soft-Probe-SECM on melanoma and normal skin thick tissue using a soft probe (Fig.5.8a) and spider probe after the electrodes brushing over it and making approach curves on it (Fig.5.8b). The specificity of the detection strategy was achieved by using first primary Abs followed by secondary Abs with HRP label. The delicate tissues remained intact and undamaged as no damages or scratches were identified on the tissues from a microscopic inspection after the SECM measurements. Generally, such tissue samples were several millimeters in thickness with a very versatile topography. In Fig.5.9a and d, the SECM maps of the distribution of the two immuno-labeled biomarkers S100 protein and tyrosinase inside the same tumor block were created. In both figures, the currents over melanoma tissues were higher, indicating the higher expression of the according biomarker. Unfortunately, the cancer stage was not identified by the tissue supplier, but based on the results from the previous chapter the melanoma tumor progression is probably below stage III due to its relative homogeneous tyrosinase distribution. Furthermore, thick tissue samples of different progression stages for comparison purposes were not available during the studies. The optical pictures of the samples after imaging are displayed in Fig.5.9b-c. In Fig.5.9c, a commercial solution contained the TMB which reacts with HRP labeled on the tissue at the locations where biomarkers presented before SECM experiment (details please see Chapter 4). The formation of light blue color appeared in the melanoma tissue indicated optically the presence of immune-labeled HRP due to the product of enzymatic reaction of TMB catalyzed by HRP in presence of $\mathrm{H}_{2} \mathrm{O}_{2}$, which is in good agreement with the SECM imaging results. Please note that the degree of natural pigmentation, and thus the color of the tissue, in melanoma of the same stage can be different. The brown spots in Fig.5.9c were mostly caused by melanin, which presence can lead to interference of the biomarker detection when using colorimetric assays. In addition, the lateral resolution of colorimetric assays based on color dyes such as TMB may suffer from the diffusion and therefore, the lateral information about the biomarker distribution may get partially lost. S100 protein and tyrosinase were both reported as biomarkers for melanoma diagnosis. ${ }^{[79,136]}$ The expression of various melanoma biomarkers can be very different depending on the stages and different genetic mutations in patients. ${ }^{[79,90,135,213-223]}$ In this case, tyrosinase over-expression level was 
higher compared with the over-expression level of S100 protein (Fig.5.9a and d), and thus tyrosinase was chosen for further study.

a)

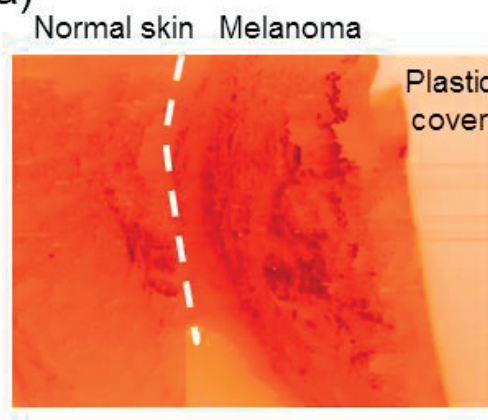

$0.5 \mathrm{~mm}$

d)

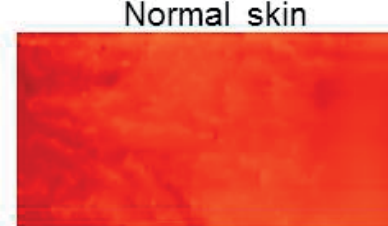

$0.2 \mathrm{~mm}$ b)

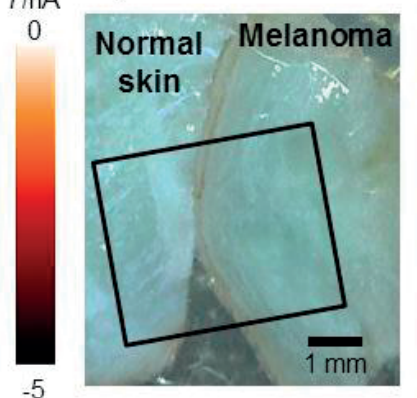

$-5$ c)

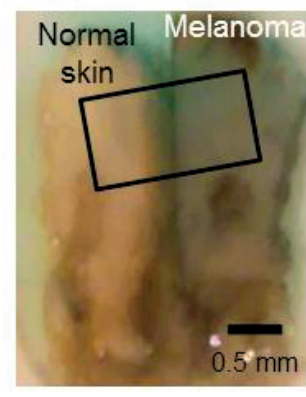

Melanoma

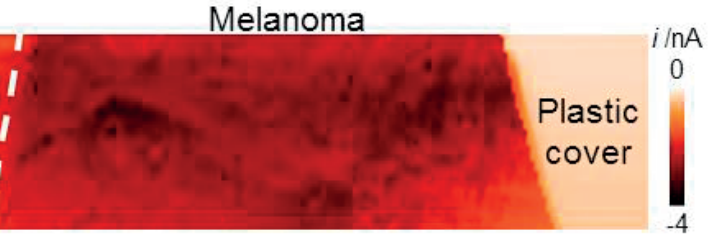

/nA

Fig.5.9 a) SECM image of S100 protein distribution using immunoassay. b) Photograph of the samples scanned in (a) after the experiment. The area scanned was labeled in the rectangular. c)TMB treated normal and melanoma tissue labeled with HRP. The area scanned in (d) is labeled as a rectangular. d) SECM image of tyrosinase distribution in normal tissue and melanoma using immunoassay. All tissues were in $2 \mathrm{mM} \mathrm{FcMeOH}, \mathrm{H}_{2} \mathrm{O}_{2} 1 \mathrm{mM}$ and $10 \mathrm{mM}$ phosphate buffer, $\mathrm{pH}$ 6. Experimental conditions: $h_{\mathrm{P}}=-300 \mu \mathrm{m}$ versus a plastic cover next to the tissues, $E_{\mathrm{T}}=-0.2 \mathrm{~V} v s$ Ag-QRE, step size $=25 \mu \mathrm{m}$, translation speed $=50 \mu \mathrm{m} / \mathrm{s}$. 
5.3.3 Thick melanoma tissue scanning by spider probe

a)

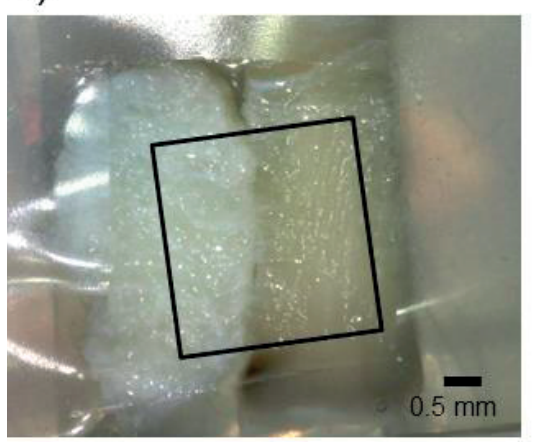

b) Normal skin Melanoma

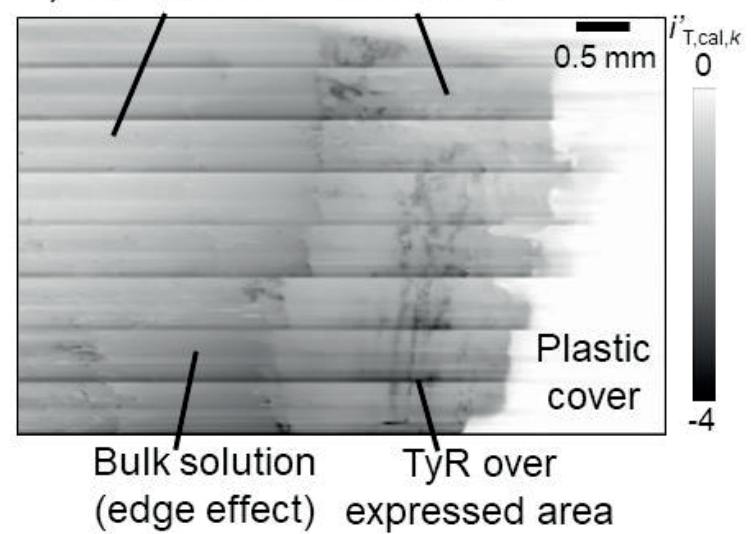

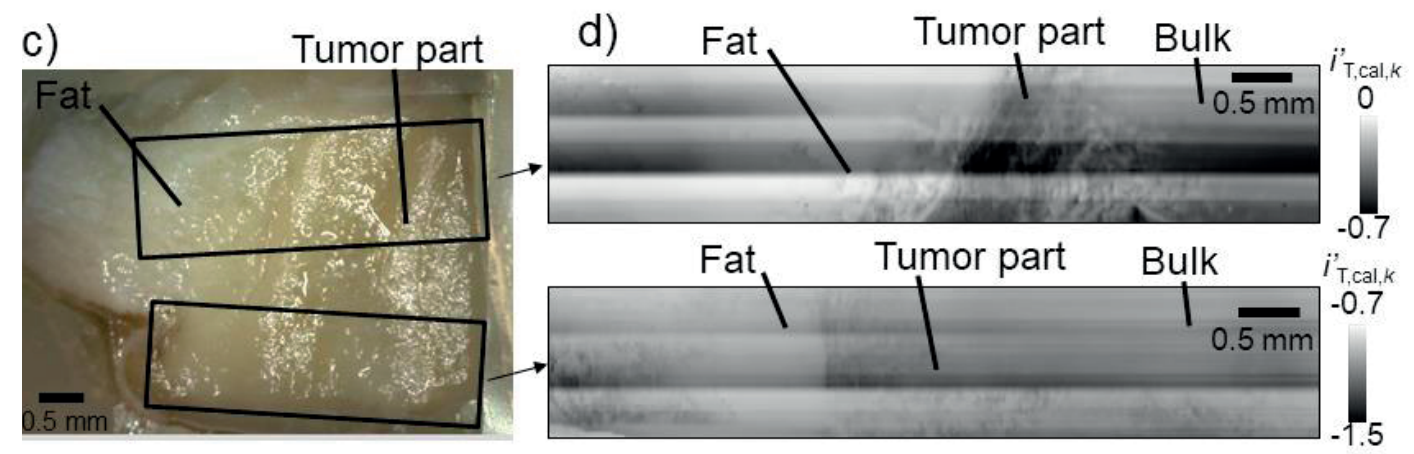

Fig.5.10 SECM SG/TC images of the tyrosinase distribution using a spider probe. a) Photograph of normal skin and melanoma after SECM scanning. b) SECM image. c) Photograph of a large melanoma sample containing fat and tumor parts recorded after SECM imaging. d) SECM images. Experimental conditions: $2 \mathrm{mM} \mathrm{FcMeOH}, \mathrm{H}_{2} \mathrm{O}_{2} 1 \mathrm{mM}$ and 10 $\mathrm{mM}$ phosphate buffer, $\mathrm{pH} 6 . h_{\mathrm{P}}=-300 \mu \mathrm{m}, E_{\mathrm{T}}=-0.2 \mathrm{~V} v s$ Ag-QRE, step size $=25 \mu \mathrm{m}$, translation speed $=50 \mu \mathrm{m} / \mathrm{s}$. tyrosinase was labeled with the immunoassay strategy. The areas scanned are indicated as black rectangles. Parameters for current calibration is in "Additional information for normalization of the SECM images".

Thick human skin tissues including one normal skin and two melanoma samples from three different people were analyzed by using a spider probe. These three samples were labeled with the tyrosinase-specific immunoassay and fixed in an electrolytic cell (Fig.5.10a and c). A frame made of plastic was placed on the tissues to fix the tissues and to provide a smooth surface for approach curves performed prior to each line scan. The positioning and movements of the eight spider legs were analyzed live with a microscope USB camera that was directed from the side through a transparent SECM cell. When the probe passed the gap between plastic cover and the tissue, each "leg" 
flipped over the gap at slightly different times. This edge effect became obvious in SECM image for each ME. In Fig.5.10b, the tyrosinase overexpression in melanoma compared to normal skin is indicated by darker color in SECM images. The tyrosinase overexpressed part (dark color) represents the tumor cell distribution. The overexpressed part is heterogeneously distributed and thus not homogenous compared with the results obtained from Fig.5.9d. This is probably a result of the different tumor cell distribution patterns. The current value in Fig.5.10b and d were calibrated by the following equations:

\section{a) Calibration of the current values}

Previously, the latter step was performed for positive feedback (e.g. CNT deposited on plastic), which was not possible to observe on the tissue samples analyzed. In contrast, negative feedback is obtained on the plastic cover placed next to the tissues. The detailed parameter is listed in the Table 5.5 and Table 5.6.

Calibrated currents $i_{\mathrm{T}, \mathrm{cal}, k}$ by applying $i_{\mathrm{T}, \mathrm{offs}, k}$ and $s_{\mathrm{k}}$ :

$i_{\mathrm{T}, \mathrm{cal}, k}=\left(i_{\mathrm{T}, k}-i_{\mathrm{T}, \mathrm{offs}, k}\right) \cdot s_{k}$

(Equation 5.10)

$i_{\mathrm{T}, \mathrm{off} s, k}-$ offset current to level the electrode responses for the microelectrode $k$. $s_{k}-$ scale factor to level the electrode responses for the microelectrode $k$ :

\section{b) Calibration of positional offsets}

$x_{k}^{\prime}=x_{k}-x_{\mathrm{offs}, k}$

$y_{k}^{\prime}=y_{k}-y_{\text {offs, }, k}$

Another effect should be taken into consideration. When the spider probe "brushed" over the edges of different tissues, it may lose contact temporarily (depending on how much the soft probe is pressed against the substrate and how deep is the surface), and suspend in the bulk solution for a short time (Fig.5.11). Therefore, the edges of different materials in the SECM image may be of jagged shape or as bulk current. In general, if the probe is flipping off a sample or climbing up a sample about significant heights (e.g. several ten $\mu \mathrm{m})$ the SECM image can locally be slightly compressed or expanded. ${ }^{[71]}$

In Fig.5.10c and d, a single melanoma thick tumor tissue obtained from another patient was labeled with the tyrosinase-specific immunoassay with HRP label and 
scanned with the spider probe. The sample was larger and contained a tumor part and a subcutaneous fat part composed of adipocytes. Indeed, the tumor part with high current signals was clearly identified. The absolute current value of the SECM image may slightly change due to the concentration of the accumulated $\mathrm{FcMeOH}^{+}$in the solution produced by HRP. In fact, a higher background signal was observed over time that could be related to the increased concentration of $\mathrm{FcMeOH}^{+}$in the solution bulk. Compared with the TMB substrate used in the previous chapters for SECM imaging, $\mathrm{FcMeOH}^{+}$ exhibited higher stability and less electrode passivation so it was chosen as the substrate in this chapter.

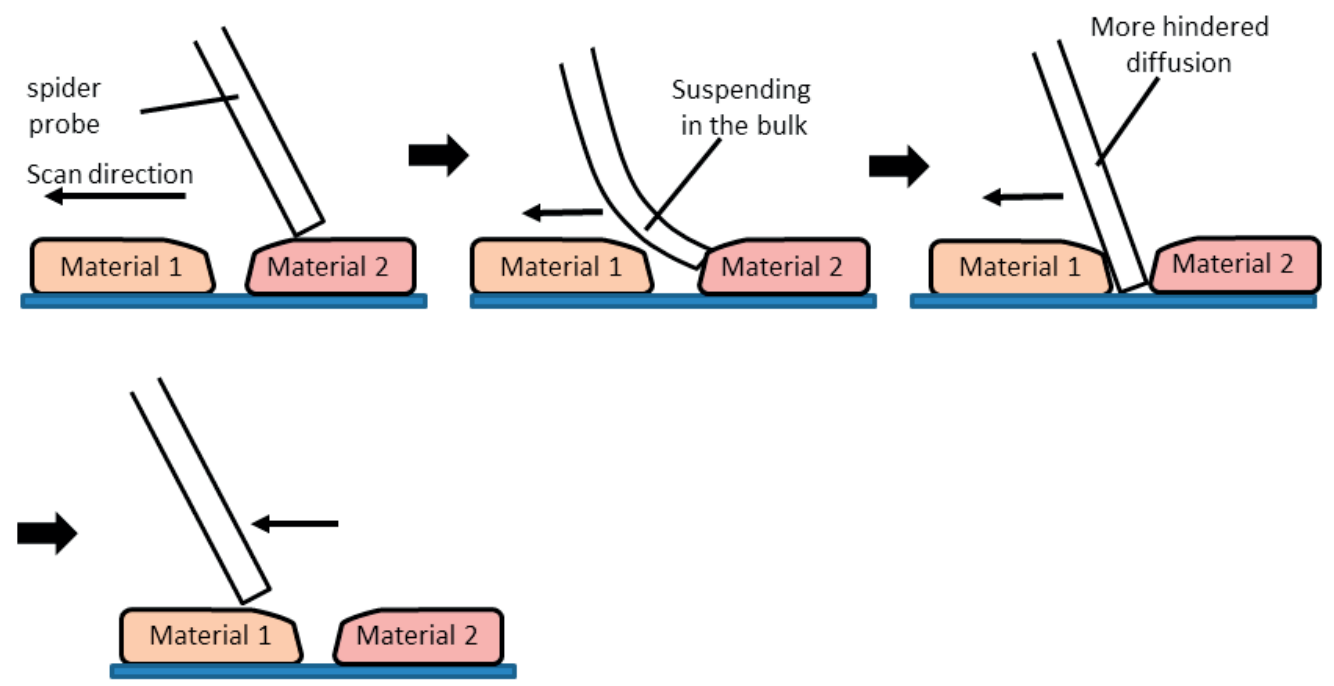

Fig.5.11 Schematic representation of a soft probe microelectrode that scans over two thick samples of different shape and dimensions.

\subsubsection{Scanning an entire mouse heart section}

In addition to melanoma samples, the ability of entire mouse organ scanning with a spider probe was tested. The heart section was attached to a microscopic glass slide. The main components of a heart are various proteins that exist in cardio vascular muscles and blood vessels. Some of the proteins can be redox active, such as hemoglobin. ${ }^{[224,225]}$ This could be used for electrochemical detection by the regeneration of a redox mediator typically used for SECM feedback mode imaging. In order to test the ability of certain relevant proteins to provide a redox mediator regeneration, $\mathrm{Hb}$ and $\mathrm{BSA}$ were deposited on a PVDF membrane and scanned by 
SECM in feedback mode using $\mathrm{FcMeOH}$ as redox mediator. $\mathrm{Hb}$ is an iron containing protein with a porphyrin ring in the center. The heme-Fe(III/II) inside $\mathrm{Hb}$ may be in oxidation state II and the $\mathrm{FcMeOH}^{+}$generated at the tip could be reduced back to $\mathrm{FcMeOH}$ causing an increased SECM current when a microelectrode is placed to $\mathrm{a} \mathrm{Hb}$ spot (Fig.5.12a). ${ }^{[226]}$ The $E^{\circ}$ for the $\mathrm{FcMeOH} / \mathrm{FcMeOH}^{+}$redox couple in PBS buffer, $\mathrm{pH} 7$ is around $0.44 \mathrm{~V} v s . \mathrm{SHE}(\sim 0.241 \mathrm{~V} v s . \mathrm{Ag} / \mathrm{AgCl}),{ }^{[227]}$ and $E^{\circ}$ for heme-Fe(III/II) inside $\mathrm{Hb}$ is around $-0.37 \mathrm{~V}$ vs. $\mathrm{Ag} / \mathrm{AgCl}$ in blood, $\mathrm{pH} 7 .^{[228]}$ Thus, the reaction $\mathrm{FcMeOH}^{+}+$heme-Fe(II) $\rightarrow \mathrm{FcMeOH}+$ heme-Fe(III) is possible depending also on the reaction kinetics and stability of the reactants and products. As for another redox couple $\left[\mathrm{Ru}\left(\mathrm{NH}_{3}\right)_{6}\right]^{3+/ 2+}$ in PBS buffer, $\mathrm{pH} 7$, the standard redox potential is around $0.05 \mathrm{~V} v s$. SHE ( - $0.149 \mathrm{~V}$ vs. Ag/AgCl). Thus heme-Fe(II) can be oxidized to heme-Fe(III) in $\left[\mathrm{Ru}\left(\mathrm{NH}_{3}\right)_{6}\right]^{3+}$ redox mediator solution and won't result in redox mediator regeneration. Fig.5.12 displays the SECM images of protein spots on PVDF membrane scanning in FcMeOH (Fig.5.12a) and $\left[\mathrm{Ru}\left(\mathrm{NH}_{3}\right)_{6}\right]^{3+}$ (Fig.5.12b) containing electrolyte solutions. Bovine serum albumin (BSA) is a serum albumin protein and it is not a metalloprotein so the current signal is weaker over the BSA spot. In Fig.5.12b, the protein spots resulted both in very low feedback currents. This indicates that both proteins have less interaction with $\left[\mathrm{Ru}\left(\mathrm{NH}_{3}\right)_{6}\right]^{3+}$. However, the clear mechanism is still unknown.

\section{a) $\mathrm{FcMeOH}$}

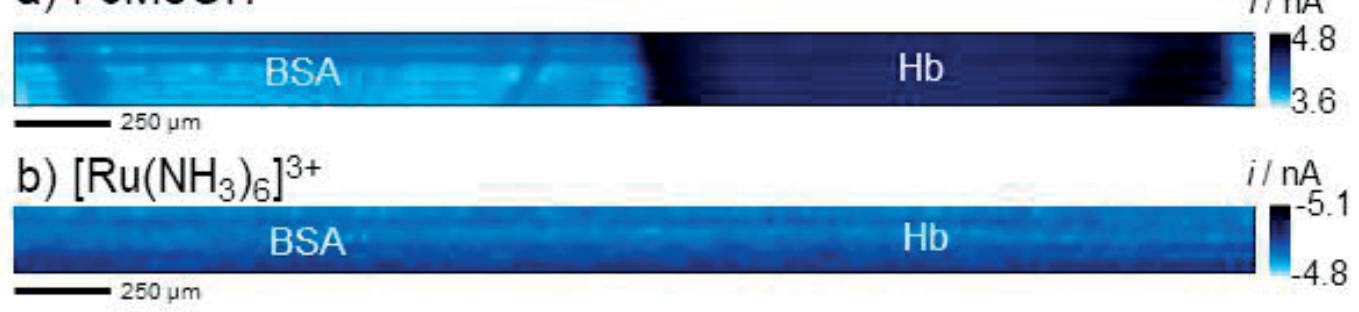

Fig.5.12 SECM image of BSA and $\mathrm{Hb}$ protein spots $(1 \mathrm{mg} / \mathrm{mL})$ on PVDF membrane in a) 2 $\mathrm{mM} \mathrm{FcMeOH}$ and b) $2 \mathrm{mM}$ of $\left[\mathrm{Ru}\left(\mathrm{NH}_{3}\right)_{6}\right]^{3+}$ scanning by soft probe in contact mode. Experimental conditions: Ag-QRE, step size $=25 \mu \mathrm{m}$, translation speed $50 \mu \mathrm{m} / \mathrm{s}, \mathrm{E}=0.4 \mathrm{~V}$ for FcMeOH and $-0.4 \mathrm{~V}$ for $\left[\mathrm{Ru}\left(\mathrm{NH}_{3}\right)_{6}\right]^{3+}$. All the solutions contain phosphate buffer $50 \mathrm{mM}, \mathrm{pH}$ 6.0 .

Fig.5.13a displays an optical microscopic picture of a slice of a mouse heart with a thickness of $\sim 15 \mu \mathrm{m}$. This sample was imaged using Soft-Probe-SECM with a spider probe of eight microelectrdoes (Fig.5.13b). The sample area was larger than what the 
soft array probe width can cover without causing double scanning of areas that were already scanned by seven out of the eight microelectrodes. Such distance in $y$-direction of an image is $4 \mathrm{~mm}$ considering the standard SECM array dimensions. If larger areas are to be scanned, the employed SECM setup enables to image a first image frame and then to place the array automatically $4 \mathrm{~mm}$ further to the adjacent next image frame. ${ }^{[41,71,202]}$. The current calibration procedures were the same as Fig.5.10. In Fig.5.10b, the micro-structure of the heart including blood vessels and interventricular septum are clearly revealed. The strong current signals come most likely from the presence of a broad range of redox active proteins so that their global distribution could be mapped electrochemically. The most possible source of the redox active proteins in heart is $\mathrm{Hb}$ which is mainly located in in the blood vessel area (yellowish color in Fig.5.10b). As discussed previously, imaging such a large area by using a traditional ME (e.g. Pt embedded in a glass insulator) is generally challenging, because the working distance could vary significantly due to the topography of the tissue section. Fig.5.10c shows a second section of the mouse heart scanned in Fig.5.10b imaged this time with a single soft probe. The current contrast between tissue and glass reveals the detailed microstructure of the heart such as the texture of the myocardium. If a single soft probe is used for imaging of the entire heart, the measurement procedure could take at about 8 times more than using the spider probe. Compared with the optical microscope images (Fig.5.10a), SECM imaging can be used for transparent samples like tissue slices or samples containing a color pigments. The porosity and permeability of the tissue is unknown. However, it can be assumed that the permeability and porosity between melanoma tissue and normal skin tissue are similar. This problem will be further investigated in the future. 
a)

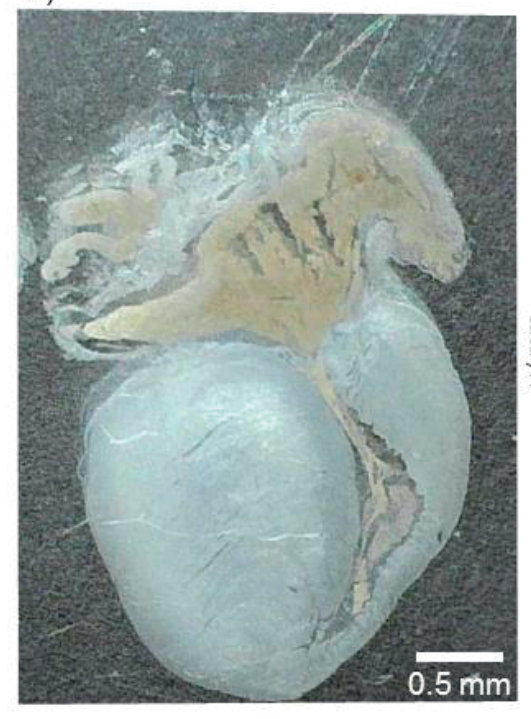

b)

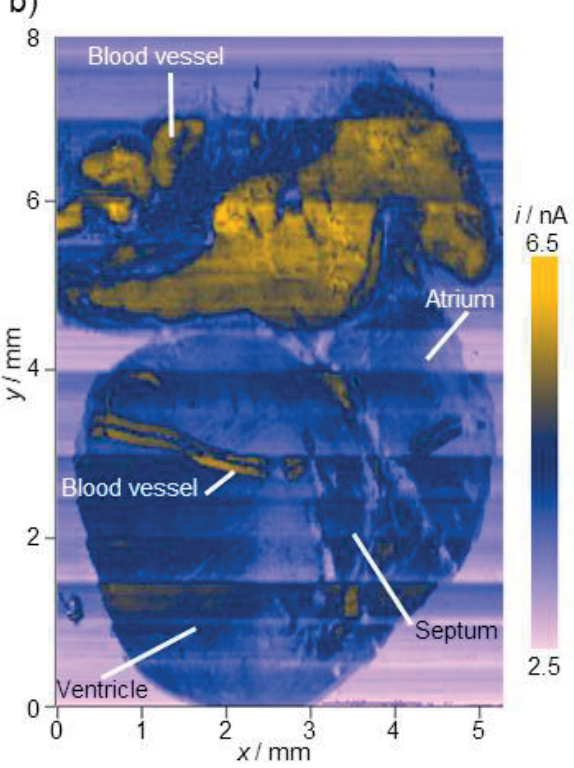

c)

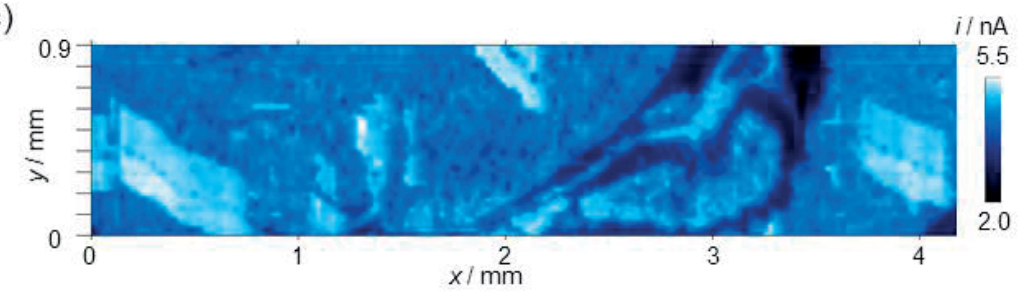

Fig.5.13 a) Optical image of a mouse heart after SECM imaging. b) SECM image of a mouse heart scanned in contact mode by soft probe. c) The detail structure of a mouse heart. The heart is the same as (b) but the section is different. Experimental conditions: Imaging time $\sim 4$ hs, 2 $\mathrm{mM}$ FcMeOH and phosphate buffer $10 \mathrm{mM}$, pH 6.0Ag-QRE, step size $=25 \mu \mathrm{m}$, translation speed $50 \mu \mathrm{m} / \mathrm{s}, \mathrm{E}=0.4 \mathrm{~V}$. The current calibration method is the same as Fig. 5.12a. Details of parameters for calibration is listed in Table 5.7.

\subsection{Conclusion}

A soft spider probe composed of eight individual microelectrodes was fabricated through laser ablation followed by laser cutting. The length and the dimension of the spider legs were optimized thus the spider array can scan artificial 3D samples with relative large topographic features. These probes were successfully applied to image thick cancer tissues. Thanks to the weak forces exerted onto the sample surface and due to the bending properties of the soft spider probe, a constant working distance was easily achieved by contact mode scanning without inducing damages, neither to the tissues nor to the probes. Furthermore, a spider probe has been used for 
imaging the redox active $\mathrm{Hb}$ proteins in the entire mouse heart section. The current over blood vessels are higher than over the myocardium part. This is possibly due to the strong signal from $\mathrm{Hb}$. Finally, in the future, the spider probe will be tested on the alive animal such as mouse skin. The micro-environment of tissue surface can be manipulated by applying high voltages and the cancer cells can be killed due to the electronic proration. The long-term goal is to develop a cancer scanning/ treatment SECM

\subsection{Additional information for normalization of the SECM images}

Table 5.1. Current offsets and scale factors of the individual electrodes in Fig.5.5b.

\begin{tabular}{ccccccccc}
\hline $\begin{array}{c}\text { Electrode } \\
\text { no. } \boldsymbol{k}\end{array}$ & $\mathbf{1}$ & $\mathbf{2}$ & $\mathbf{3}$ & $\mathbf{4}$ & $\mathbf{5}$ & $\mathbf{6}$ & $\mathbf{7}$ & $\mathbf{8}$ \\
\hline iт, off, $\boldsymbol{k}$ & 0 & 0 & 0 & 0 & 0 & 0 & -1.5 & -2 \\
$\boldsymbol{s}_{\boldsymbol{k}}$ & 1 & 1 & 1 & 1 & 1 & 1 & 1 & 1 \\
$\boldsymbol{x}_{\text {offset }}$ & 0 & 0 & 0 & 0 & 0 & 0 & 0 & 0 \\
$\boldsymbol{y}_{\text {offset }}$ & 0 & 500 & 1000 & 1500 & 2000 & 2500 & 3000 & 3500 \\
$(\mu \mathrm{m})$ & & & & & & & &
\end{tabular}

Table 5.2 Current offsets and scale factors of the individual electrodes in Fig.5.5d

\begin{tabular}{ccccccccc}
\hline $\begin{array}{c}\text { Electrode } \\
\text { no. } \boldsymbol{k}\end{array}$ & $\mathbf{1}$ & $\mathbf{2}$ & $\mathbf{3}$ & $\mathbf{4}$ & $\mathbf{5}$ & $\mathbf{6}$ & $\mathbf{7}$ & $\mathbf{8}$ \\
\hline $\mathbf{i}$, off, $\boldsymbol{k}$ & -1.5 & 0 & -2 & 0 & -2 & 0 & 0 & 0 \\
$\boldsymbol{s}_{\boldsymbol{k}}$ & 1 & 1 & 1 & 1 & 1 & 1 & 1 & 1 \\
$\boldsymbol{x}_{\text {offset }}$ & 0 & 0 & 0 & 0 & 0 & 0 & 0 & 0 \\
$\boldsymbol{y}_{\text {offset }}$ & 0 & 500 & 1000 & 1500 & 2000 & 2500 & 3000 & 3500 \\
$(\mu \mathrm{m})$ & & & & & & & &
\end{tabular}

Table 5.3 Current offsets and scale factors of the individual electrodes in Fig.5.6c.

\begin{tabular}{lllllllll}
\hline Elect & 1 & 2 & 3 & 4 & 5 & 6 & 7 & 8 \\
\hline
\end{tabular}




\begin{tabular}{|c|c|c|c|c|c|c|c|c|}
\hline $\begin{array}{l}\text { rode } \\
\text { no. } k\end{array}$ & & & & & & & & \\
\hline iT, off, $k$ & 4.20 & 4.50 & 3.65 & 4.20 & 3.70 & 4.30 & 9.00 & 3.20 \\
\hline \multirow{2}{*}{$s_{k}$} & \multirow{2}{*}{0.45454} & 0.7692 & 1.0000 & 0.6896 & 1.100 & 0.3636 & 0.6000 & 1.0000 \\
\hline & & 3 & 0 & 5 & 00 & 3 & 0 & 0 \\
\hline$x_{\text {offset }}$ & 0 & 0 & 0 & 0 & 0 & 0 & 0 & 0 \\
\hline $\begin{array}{l}\boldsymbol{y}_{\text {offset }} \\
(\mu \mathrm{m})\end{array}$ & 0 & 500 & 1000 & 1500 & 2000 & 2500 & 3000 & 3500 \\
\hline
\end{tabular}

Table 5.4 Current offsets and scale factors of the individual electrodes in Fig.5.6d.

\begin{tabular}{ccccccccc}
\hline $\begin{array}{r}\text { Electro } \\
\text { de no. } \boldsymbol{k}\end{array}$ & $\mathbf{1}$ & $\mathbf{2}$ & $\mathbf{3}$ & $\mathbf{4}$ & $\mathbf{5}$ & $\mathbf{6}$ & $\mathbf{7}$ & $\mathbf{8}$ \\
\hline $\mathbf{i}$ T, off, $\boldsymbol{k}$ & 5.90 & 5.50 & 6.60 & 5.25 & 6.20 & 5.00 & 5.60 & 5.50 \\
$\boldsymbol{s}_{\boldsymbol{k}}$ & 0.3333 & 0.1328 & 0.3333 & 0.1000 & 0.1250 & 0.3636 & 0.2500 & 0.8000 \\
$\boldsymbol{x}_{\text {offset }}$ & 3 & 5 & 3 & 0 & 0 & 4 & 0 & 0 \\
$\boldsymbol{y}_{\text {offset }}$ & 0 & 0 & 0 & 0 & 0 & 0 & 0 & 0 \\
$(\mu \mathrm{m})$ & 0 & 500 & 1000 & 1500 & 2000 & 2500 & 3000 & 3500 \\
\hline
\end{tabular}

Table 5.5 Current offsets and scale factors of the individual electrodes in Fig.5.10b

\begin{tabular}{ccccccccc}
\hline $\begin{array}{c}\text { Electrode } \\
\text { no. } \boldsymbol{k}\end{array}$ & $\mathbf{1}$ & $\mathbf{2}$ & $\mathbf{3}$ & $\mathbf{4}$ & $\mathbf{5}$ & $\mathbf{6}$ & $\mathbf{7}$ & $\mathbf{8}$ \\
\hline iт, off, $\boldsymbol{k}$ & 0 & 0 & 0 & 0 & 0 & 0 & 0 & 0 \\
$\boldsymbol{s}_{\boldsymbol{k}}$ & 1.5 & 3 & 1 & 1 & 1 & 1 & 1 & 1 \\
$\boldsymbol{x}_{\text {offset }}$ & 0 & 0 & 0 & 0 & 0 & 0 & 0 & 0 \\
$\boldsymbol{y}_{\text {offset }}$ & 0 & 500 & 1000 & 1500 & 2000 & 2500 & 3000 & 3500 \\
$(\mu \mathrm{m})$ & & & & & & & &
\end{tabular}

Table 5.6 Current offsets and scale factors of the individual electrodes in Fig.5.10d

\begin{tabular}{ccccccc}
\hline $\begin{array}{c}\text { Electrode } \\
\text { no. } \boldsymbol{k}\end{array}$ & $\mathbf{1}$ & $\mathbf{2}$ & $\mathbf{3}$ & $\mathbf{4}$ & $\mathbf{5}$ & $\mathbf{6}$ \\
\hline i, $\boldsymbol{o f f} \boldsymbol{k}$ & 0 & 0 & 0 & 0 & 0 & 0 \\
$\boldsymbol{s}_{\boldsymbol{k}}$ & 1 & 1.5 & 1 & 1.2 & 1.5 & 2 \\
\hline \multicolumn{7}{c}{108}
\end{tabular}




\begin{tabular}{ccccccc}
\hline$x_{\text {offset }}$ & 0 & 0 & 0 & 0 & 0 & 0 \\
$y_{\text {offset }}$ & 0 & 500 & 1000 & 1500 & 2000 & 2500 \\
$(\mu \mathrm{m})$ & & & & & & \\
\hline
\end{tabular}

Table 5.7 Current offsets and scale factors of the individual electrodes in Fig.5.13b

\begin{tabular}{ccccccccc}
\hline $\begin{array}{c}\text { Electrode } \\
\text { no. } \boldsymbol{k}\end{array}$ & $\mathbf{1}$ & $\mathbf{2}$ & $\mathbf{3}$ & $\mathbf{4}$ & $\mathbf{5}$ & $\mathbf{6}$ & $\mathbf{7}$ & $\mathbf{8}$ \\
\hline $\mathbf{i}_{\mathrm{T}, \text { off, } \boldsymbol{k}}$ & 0 & 0 & 0 & 0 & 0 & 0 & 0 & 0 \\
$\boldsymbol{s}_{\boldsymbol{k}}$ & 0.9 & 1.6 & 1.6 & 1 & 1 & 1 & 1 & 1.3 \\
$\boldsymbol{x}_{\text {offset }}$ & 0 & 0 & 0 & 0 & 0 & 0 & 0 & 0 \\
$\boldsymbol{y}_{\text {offset }}$ & 0 & 500 & 1000 & 1500 & 2000 & 2500 & 3000 & 3500 \\
$(\mu \mathrm{m})$ & & & & & & & &
\end{tabular}




\section{Imaging the distribution of graphene oxide nanoribbons in mice livers by soft probe SECM}

\subsection{Introduction}

A broad range of carbonaceous nanomaterials, such as carbon nanotubes, graphene and graphene oxide, is currently discussed in literature for in vivo applications, such as photothermal therapy and drug delivery. ${ }^{[110-112,229-231]}$ This is due to the large variety of adjustable material properties including NIR absorbance or enhanced drug loading capacity, respectively. However, for biomedical applications the applied nanomaterials must fulfill certain requirements: $i$ ) low toxicity $i$ ) rapid excretion, $i i i)$ high solubility in the injection medium and in the body fluids, $i v$ ) stability and biocompatibility under physiological conditions, v) knowledge about the regions in the body where the nanomaterials will accumulate and vi) low cost. Solubility in aqueous solutions can be achieved by chemically oxidizing the $\pi$-conjugated network of the CNTs or graphene to add hydrophilic functional groups. Hence, graphene oxide (GO) and its derivatives, such as GO nanoribbons (GONRs), have attracted considerable attention for in vivo applications. Graphene oxide nanoribbons are strips of GO with a high length-to-width ratio $(\sim 150)$ and can be synthesized by the longitudinal unzipping of multiwalled carbon nanotubes (MWCTs) under oxidizing conditions. ${ }^{[122,232-234]}$ Enhanced biocompatibility and stability while avoiding non-specific adsorption is achieved by the covalent or non-covalent functionalization with polyethylene glycol (PEG). ${ }^{[11]}$ Furthermore, the loading with anti-cancer drugs, such as doxorubicin (DOX), is a result of the $\pi$ - $\pi$-interactions between the drug and the GONR composite. ${ }^{[235]}$ The variety of GO derivatives and their wide range of possibilities to functionalize lead to different interactions of the nanomaterials with biological soft matter. Consequently, the accumulation region, retention time and toxicity are also affected. In order to understand the interaction of such materials with biological targets and to map the biodistribution as a result of both the nanomaterial transport in blood and the accumulation in tissue, high resolution imaging technologies need to be applied. Typical techniques to visualize the drug distribution of GO derivatives in animals and 
to study the pharmacokinetics include autoradiography, whole body single photon emission computed tomography (SPECT/CT) and positron emission tomography (PET). ${ }^{[235,236]}$ These techniques have in common that radioactive labels are used, which can be loaded onto the GO derivatives to identify the preferred location of the accumulation in the target. The measurement period after injection is restricted due to the half-life time of the radioactive labels. For autoradiography, sacrificed and cryosectioned mice are placed onto a photo film for many hours to days. On the other hand, SPECT/CT and PET is shorter and can be applied also to living samples while the resolution is limited to sub-mm. It has been shown that GONRs are mainly accumulated in the reticuloendothelial system (RES) consisting of phagocytic cells and being present for instance in liver and spleen. Bio Bioimaging reagents such as GONRs are often labeled, for instance with radio or fluorescent tagged, which creates the question for the stability of tagged nanomaterials inside animal bodies. Once these tagged nanomaterials enter the animals, the tag could separate from the nanomaterial and get into the surrounding biological fluids composed of proteins, salts and cells. In such cases, the detected signals for bio-imaging are not coming from the tagged nanomaterials, but primarily from the tags which could be located in different regions of the body. This could potentially lead to wrong conclusions about the distribution of the nanomaterials under study. Alternatively, the tagged materials can experience xenobiotic breakdown in liver involving reduction, oxidation or conjugation of hydrophilic functional groups to facilitate the excretion by urine or stool. ${ }^{[237-239]}$ The in vivo applications of nanomaterials, such as GONRs, need to address the question of toxicity. In fact, certain toxicity is discussed in literature ${ }^{[240,241]}$ while other studies have shown that the toxicity can be significantly reduced. ${ }^{[241]}$ Hence, analytical tools able to study the interaction of such materials with biological tissues is important.

In this chapter, thick and thin mouse liver tissue sections have been investigated by Soft-Probe-SECM in feedback mode using ferrocene methanol and ruthenium hexaamine as redox mediators. Prior to the sample preparation for electrochemical imaging experiments, GONRs functionalized with PEG (PEG-GONRs) and partially loaded with magnetic nanoparticles (PEG-MNP-GONRs) were injected into the mice. SECM feedback currents were recorded due to the presence of the GONR composites. The electrochemical results were compared with those obtained from autoradiography and SPECT/CT. 


\subsection{Results and discussions}

Nanomaterials with sufficient redox activity, such as the PEG-MNP-GONR inside biological tissues, can initiate the oxidation or reduction of redox active species initially present or generated in the electrolyte solution in which the tissue samples are immersed (Scheme 6.1). Hence, an electron transfer between the PEG-MNP-GONR-composite and the redox active species in solution takes place contributing to the regeneration of the redox mediator and resulting in an increased current signal at the SECM probe. In another work, the electronic conductivity of reduced GO layers was mapped by using SECM. ${ }^{[242,243]}$ Analyzed GO thin films showed conductivity compared to the inactive glass substrate. 
a)

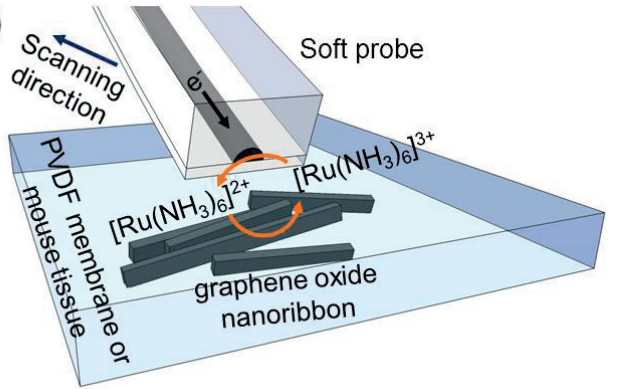

b)

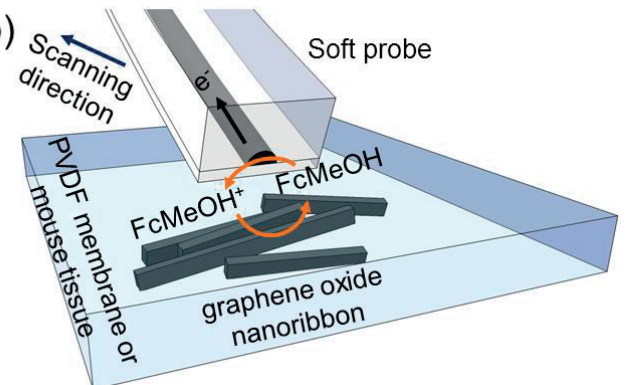

Scheme 6.1 Schematic representation of scanning GONRs in feedback mode by using a) $\left[\mathrm{Ru}\left(\mathrm{NH}_{3}\right)_{6}\right]^{3+}$ and b) FcMeOH.

In order to investigate the redox properties of the MNP-GONR composite, two 0.8 $\mu \mathrm{L}$ droplets of $2.5 \mathrm{mg} / \mathrm{mL}$ MNP-GONRs were deposited onto a PVDF membrane and imaged with Soft-Probe-SECM. Fig.6.1 displays the SECM images of the MNP-GONR spots measured in electrolyte solutions containing either $\mathrm{FcMeOH}$ (Fig.6.1a) or $\left[\mathrm{Ru}\left(\mathrm{NH}_{3}\right)_{6}\right]^{3+}$ (Fig.6.1b). The MNP-GONRs are hydrophilic whereas PVDF is hydrophobic resulting in a coffee ring drying effect resulting in an accumulation of the MNP-GONRs in a ring-like structure with undefined coverage. The ring diameters were generally about $1.5-1.8 \mathrm{~mm}$ and the ring width with higher material accumulation was up to $200 \mu \mathrm{m}$. The current increased over the MNP-GONRs indicating a redox mediator regeneration, i.e., $\mathrm{FcMeOH}^{+}+e^{-} \rightarrow \mathrm{FcMeOH}$ (reduction) and $\left[\mathrm{Ru}\left(\mathrm{NH}_{3}\right)_{6}\right]^{2+} \rightarrow$ $\left[\mathrm{Ru}\left(\mathrm{NH}_{3}\right)_{6}\right]^{3+}+e^{-}$(oxidation) at the MNP-GONRs, respectively. 

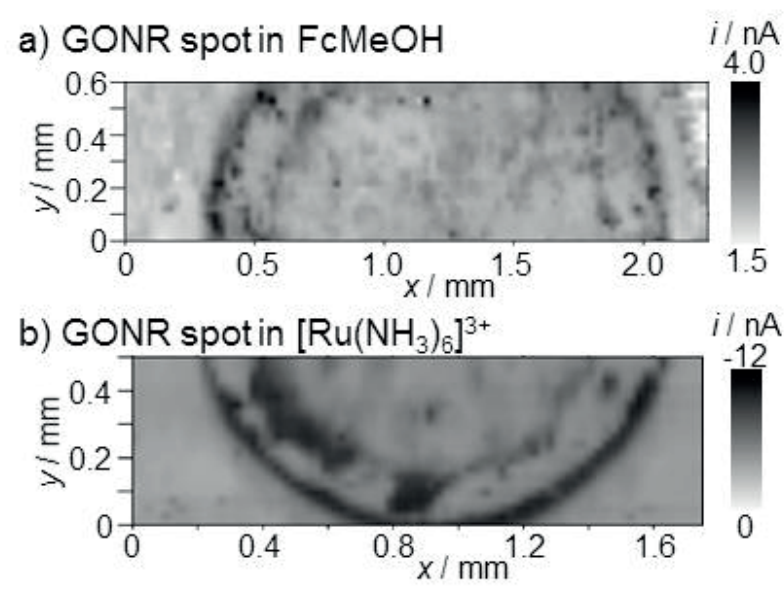

Fig.6.1 SECM image of MNP-GONR spots in a) FcMeOH and b) $\left[\mathrm{Ru}\left(\mathrm{NH}_{3}\right)_{6}\right]^{3+}$ containing electrolyte solutions scanned in contact mode with a soft probe. Experimental conditions: The concentration of MNP-GONRs was $2.5 \mathrm{mg} / \mathrm{mL}$ within a deposited volume of $0.8 \mu \mathrm{L}$ that spread in coffee-ring shape of diameter $\sim 1.5 \mathrm{~mm}$. The redox mediator concentration was $2 \mathrm{mM}$ with phosphate buffer $10 \mathrm{mM}, \mathrm{pH} 6.0, \mathrm{Ag}-\mathrm{QRE}, \mathrm{Pt} \mathrm{CE}$, step size $=25 \mu \mathrm{m}$, translation speed $50 \mu \mathrm{m} / \mathrm{s}$, $E=0.4 \mathrm{~V}$ in $\mathrm{FcMeOH}$ and $-0.4 \mathrm{~V}$ in $\left[\mathrm{Ru}\left(\mathrm{NH}_{3}\right)_{6}\right]^{3+}$.

GONRs coated with and without MNPs showed comparable current signals under the same experimental conditions indicating a minor or negligible contribution of the MNPs to the feedback current (Fig.6.2). The current over the formed ring of the spots are higher, as expected, due to the higher particle concentration as a result of the coffee ring effect. In $\mathrm{FcMeOH}$ solution, the feedback currents are higher over the spots without MNPs. The reason could be that the at the same concentration, the pure GONR spot contains more active GONRs than the MNP-GONR spot, because the nanomaterial solutions were prepared based on the full weight of the particles. On the contrary, in $\left[\mathrm{Ru}\left(\mathrm{NH}_{3}\right)_{6}\right]^{3+}$ containing solution the current over the two spots appeared more similar. This could be attributed to different reaction kinetics for the oxidation of $\left[\mathrm{Ru}\left(\mathrm{NH}_{3}\right)_{6}\right]^{3+}$. 

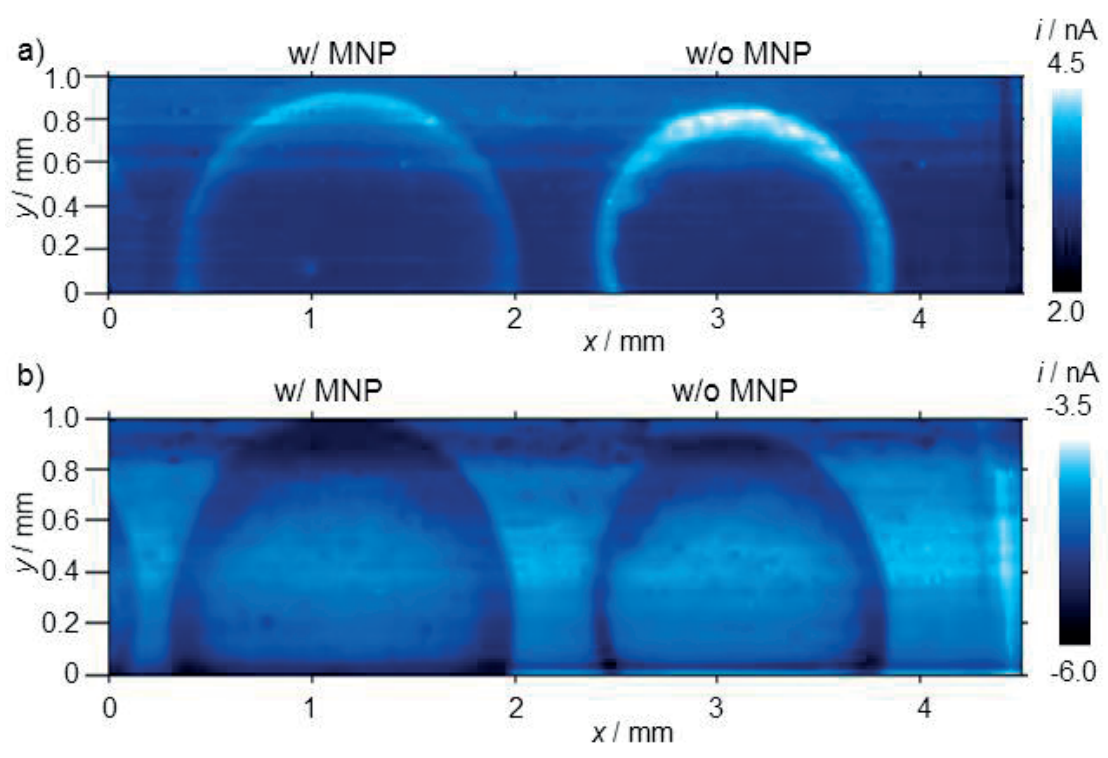

Fig.6.2 SECM image of GONR (w/ and w/o MNP) spots with concentration of $2.5 \mathrm{mg} / \mathrm{mL}$ on PVDF membrane scanned in contact mode by soft probe. a) in $2 \mathrm{mM} \mathrm{FcMeOH}, E=0.4 \mathrm{~V}$. b) in $2 \mathrm{mM}\left[\mathrm{Ru}\left(\mathrm{NH}_{3}\right)_{6}\right]^{3+}, E=-0.4 \mathrm{~V}$. Experimental conditions: $10 \mathrm{mM}$ phosphate buffer (pH 6.0), Ag-QRE, step size $=25 \mu \mathrm{m}$, translation speed $50 \mu \mathrm{m} / \mathrm{s}$.

Furthermore, the presence of PEG did not show a significant effect on the redox mediator regeneration using $\left[\mathrm{Ru}\left(\mathrm{NH}_{3}\right)_{6}\right]^{3+}$ suggesting that the PEG did not insulate fully or significantly the MNP-GONRs (Fig.6.3). 


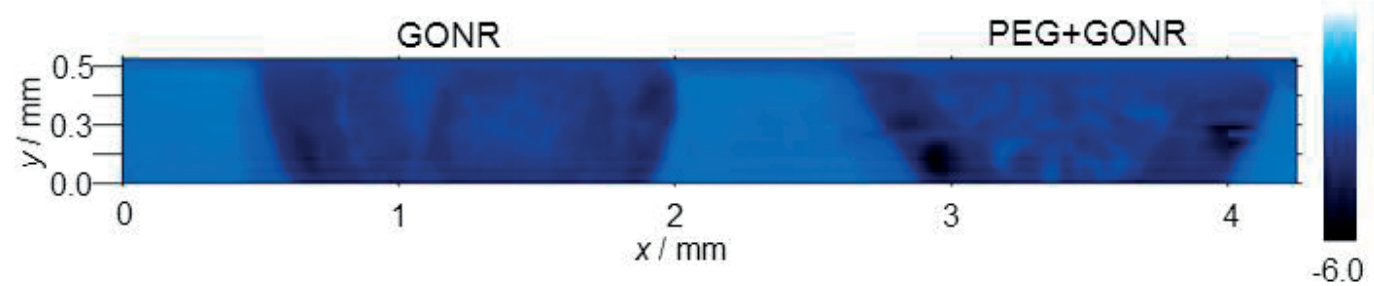

Fig.6.3 SECM image of GONR (w/ and w/o PEG) spots with concentration of $2.5 \mathrm{mg} / \mathrm{mL}$ on PVDF membrane scanned in contact mode with a soft probe in $2 \mathrm{mM}\left[\mathrm{Ru}_{(}\left(\mathrm{NH}_{3}\right)_{6}\right]^{3+}$ and phosphate buffer $10 \mathrm{mM}$ (pH 6.0). Experimental conditions: PEG with molecular weight of 1000, concentration $0.25 \mathrm{mg} / \mathrm{mL}$, was mixed with GONR solution $2.5 \mathrm{mg} / \mathrm{mL}$ and sonicated for $10 \mathrm{~min}$ before the deposition. $E=-0.4 \mathrm{~V}$ vs Ag-QRE, step size $=25 \mu \mathrm{m}$, translation speed $50 \mu \mathrm{m} / \mathrm{s}$.

In order to detect nanomaterials inserted in or deposited on substrates it is important to analyze the ranges in which they can be identified with certainty (e.g. limit of detection (LOD) or limit of quantification (LOQ)) In fact, the regeneration currents for $2 \mathrm{mM}\left[\mathrm{Ru}\left(\mathrm{NH}_{3}\right)_{6}\right]^{3+}$ showed a dependence of the MNP-GONR concentration and the MNP-GONR rings made with as low as $0.8 \mu \mathrm{L}$ of $0.2 \mathrm{mg} / \mathrm{mL}$ MNP-GONRs (i.e., $0.16 \mu \mathrm{g}$ ) were detectable (Fig.6.4). However, as already noted, the GONRs were mostly located at the rim of the deposited spots with an unknown effective coverage. This uncertainty is also a result of the heterogeneous coverage along the ring as it can be seen in the heterogeneous SECM currents. The average currents along the full diameter of the spots from the first line scan in $x$ direction (top of the figure) were found to be proportional to the concentration of MNP-GONR between the range of $0-2.5 \mathrm{mg} / \mathrm{mL}$ (Fig.6.4). The conductivity of GO and its derivatives depends on the relative number of $s p^{2}$ and $s p^{3}$ carbons. The presence of $s p^{3}$ carbons is the result of defects and holes in the $\pi$-network lowering the electronic conductivity. As shown previously, the bare and PEGylated MNP-GONRs used also in this work showed $I_{\mathrm{D}} / I_{\mathrm{G}}$ ratios of 0.94 and 0.96 , respectively, ${ }^{[235]}$ which are similar to that typically reported for reduced GO (rGO) that is considered as a material with considerable conductivity. ${ }^{[244]}$ Although such comparison must be made cautiously, it confirms that the present MNP-GONR composite shows certain conductivity as suggested by the SECM feedback images obtained with both redox mediators. Notably, the regeneration currents recorded with $\left[\mathrm{Ru}\left(\mathrm{NH}_{3}\right)_{6}\right]^{3+}$ were significantly higher than for $\mathrm{FcMeOH}$ indicating faster kinetics also 
when considering the contribution of oxygen reduction to the reduction current of $\left[\mathrm{Ru}\left(\mathrm{NH}_{3}\right)_{6}\right]^{3+}$ that is fairly low at carbon paste. ${ }^{[244]}$

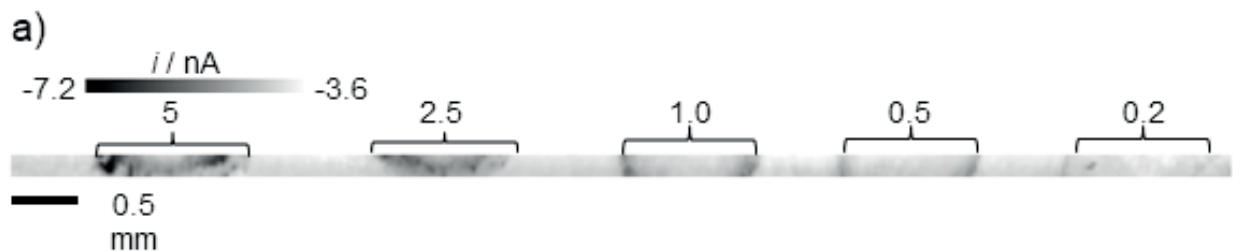

b)

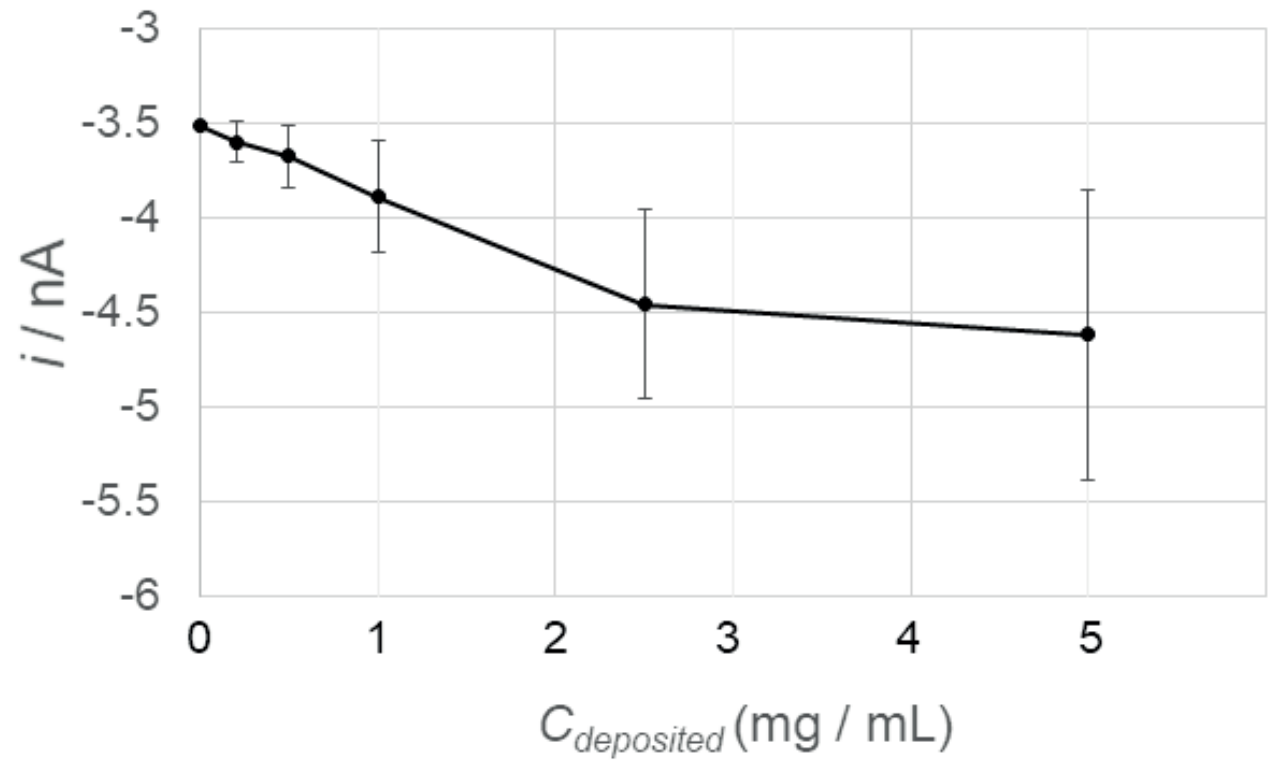

Fig.6.4 a) SECM image of MNP-GONR spots made with $0.8 \mu \mathrm{L}$ with different concentrations $c_{\text {deposited }}(5,2.5,1.0,0.5,0.2 \mathrm{mg} / \mathrm{mL})$ on PVDF membrane scanned in contact mode with a soft probe. b) Plot of average currents over the spot versus and $c_{\text {deposited. }}$ Experimental conditions: 2 $\mathrm{mM}\left[\mathrm{Ru}\left(\mathrm{NH}_{3}\right)_{6}\right]^{3+}$ with $10 \mathrm{mM}$ phosphate buffer $(\mathrm{pH}$ 6.0), Ag-QRE, step size $=25 \mu \mathrm{m}$, translation speed $50 \mu \mathrm{m} / \mathrm{s}, E=-0.4 \mathrm{~V}$.

Fig.6.5 displays SECM feedback images of thin liver tissue sections (thickness ranging approx. from 15 to $20 \mu \mathrm{m}$ ) on glass from PEG-MNP-GONR-injected mice and GONR-free mice using separately the two different redox mediators $\mathrm{FcMeOH}$ and $\left[\mathrm{Ru}\left(\mathrm{NH}_{3}\right)_{6}\right]^{3+}$. The tissue samples were comparable in terms of the location of their removal from the mouse organ and their preparation procedure. The GONR concentration in the solution for the injection into the mice was identical. During all contact mode line scans, the tissue samples were well adhered and stayed fully intact as no scratches were identified on the tissue sections after the experiments. The recorded currents were normalized by the steady state current measured in the solution 
bulk. In all cases, the contrast between tissue and inert glass substrate can be seen clearly. Even without GONRs, a higher feedback current was detected over the tissues than over the inert glass (Fig.6.5 a and c). This could be due to the presence of redox active proteins, such as thioredoxin present in liver, ${ }^{[245]}$ inducing possible redox mediator regeneration. ${ }^{[246]}$ Furthermore, the SECM current can be higher over the tissue compared to the situation on flat glass due to possible redox mediator permeability of the tissue or porosity of the sample causing less blocking of the diffusion of the redox mediator from the bottom. ${ }^{[12]}$ However, the contribution of these effects, for instance, the diffusion length of the redox mediator inside the tissue, is still unclear and need to be further analyzed. In addition, the contribution of these effects is probably different for $\mathrm{FcMeOH}$ and $\left[\mathrm{Ru}\left(\mathrm{NH}_{3}\right)_{6}\right]^{3+}$ due to different hydrophobicity (i.e., lower for $\left.\left[\mathrm{Ru}\left(\mathrm{NH}_{3}\right)_{6}\right]^{3+}\right)$. Furthermore, the regeneration will be influenced by the redox potentials and reaction kinetics. The currents over the tissues in Fig.6.5a were higher than Fig.6.5c. This is due to the presence of redox active proteins, for instance, hemoglobin, that can lead to the regeneration of $\mathrm{FcMeOH}$. In Chapter 5, this phenomenon is discussed in detail.

Topographic features in the SECM currents can be seen when the soft probe of $500 \mu \mathrm{m}$ width was slid over the edge of the tissue (Fig.6.5d, white circle). When the edge of the soft probe tip was in contact with the tissue, but the active microelectrode area, which is located in this case approximately $20 \mu \mathrm{m}$ away in the center of the probe tip, was positioned freely suspended in solution beyond the tissue surface a larger current due to the pronounced diffusion of the redox mediator from the bottom was recorded. This current is a result of the $h_{\mathrm{P}}$ value which is identical to the substrate thickness (Fig.6.5). 
a) GONR(-), FcMeOH

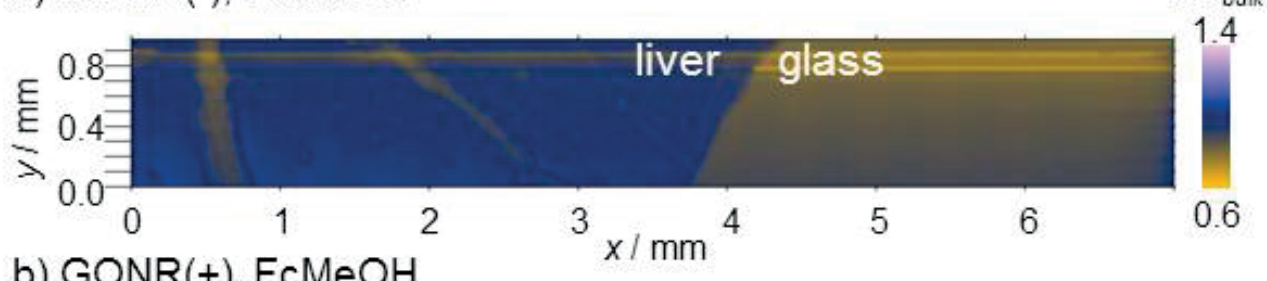

b) $\mathrm{GONR}(+), \mathrm{FcMeOH}$

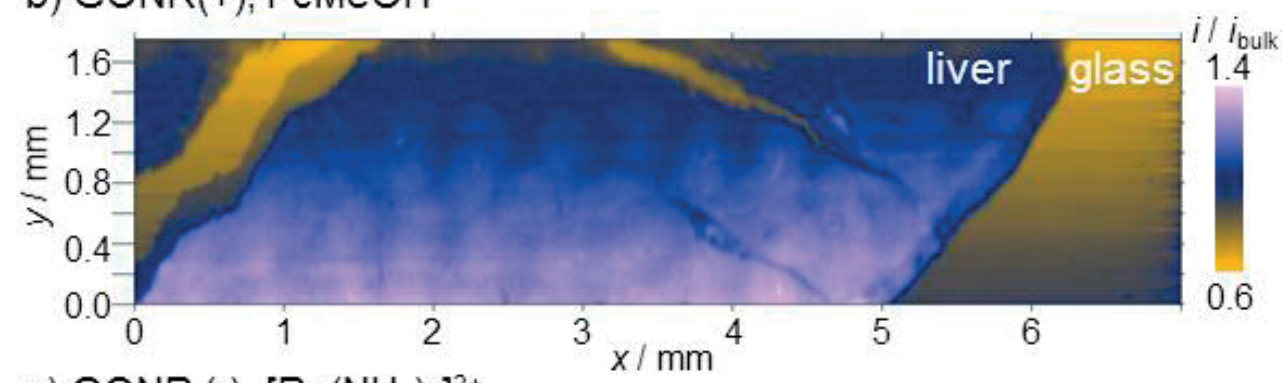

c) $\operatorname{GONR}(-),\left[\mathrm{Ru}\left(\mathrm{NH}_{3}\right)_{6}\right]^{3+}$

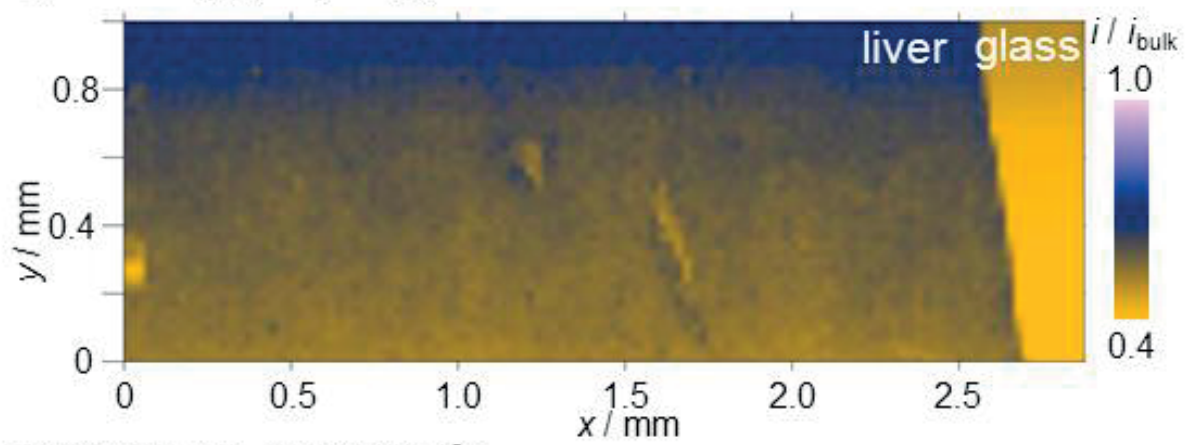

d) $\operatorname{GONR}(+),\left[\mathrm{Ru}\left(\mathrm{NH}_{3}\right)_{6}\right]^{3+}$

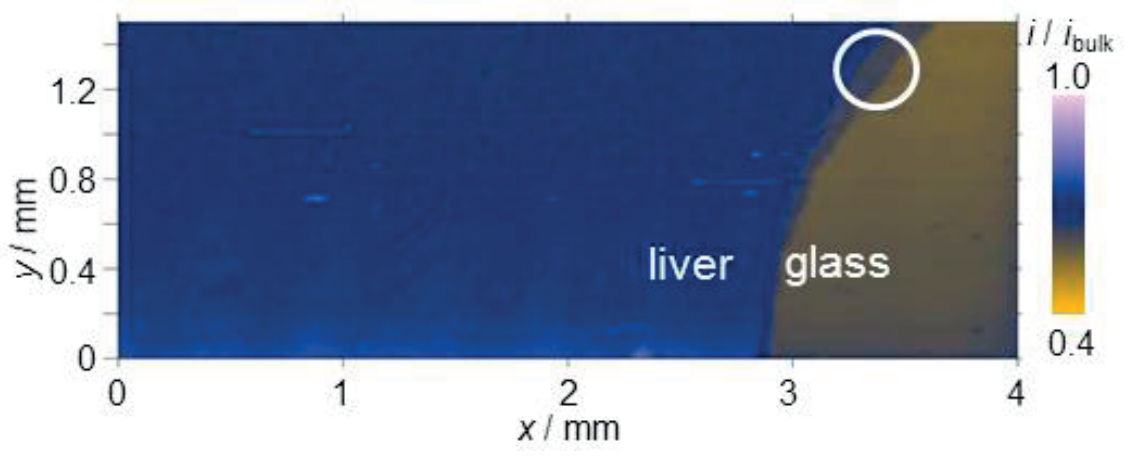

Fig.6.5 a) and c) SECM images of mouse livers without PEG-MNP-GONR injection scanned in $\mathrm{FcMeOH}$ and $\left[\mathrm{Ru}\left(\mathrm{NH}_{3}\right)_{6}\right]^{3+}$. b) and d) SECM images of mouse livers with PEG-MNP-GONR injection in scanned in $\mathrm{FcMeOH}$ and $\left[\mathrm{Ru}\left(\mathrm{NH}_{3}\right)_{6}\right]^{3+}$ Experimental conditions: $2 \mathrm{mM}$ FcMeOH or $\left[\mathrm{Ru}\left(\mathrm{NH}_{3}\right)_{6}\right]^{3+}$ with phosphate buffer $10 \mathrm{mM}$, pH 6.0, Ag-QRE, step size $=25 \mu \mathrm{m}$, translation speed $50 \mu \mathrm{m} / \mathrm{s}, E=0.4 \mathrm{~V}$ for $\mathrm{FcMeOH}$ and $-0.4 \mathrm{~V}$ for $\left[\mathrm{Ru}\left(\mathrm{NH}_{3}\right)_{6}\right]^{3+}$. All the samples were scanned in contact mode with a soft probe.

In order to exclude any influence from redox active compounds that could be present and released from the tissue over time and could be detected by the 
microelectrode, Soft-Probe-SECM of two mice livers without GONRs were subsequently imaged in pure phosphate buffer applying the potential typically applied for $\mathrm{FcMeOH}$ and $\left[\mathrm{Ru}\left(\mathrm{NH}_{3}\right)\right]_{6}{ }^{3+}$, respectively. The recorded currents over the liver and glass were homogeneous and notably low (i.e., few pA) in both cases (Fig.6.6a-b). This confirms that no direct electrochemical detection of biological compounds at the microelectrode took place.

a) $\mathrm{PB}, 0.4 \mathrm{~V}$

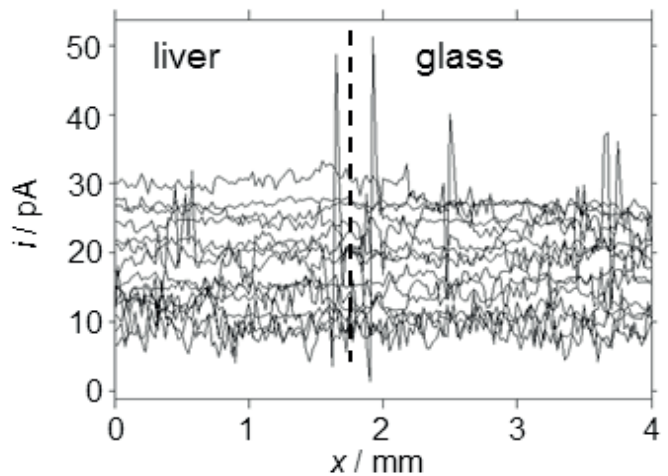

b) $\mathrm{PB},-0.4 \mathrm{~V}$

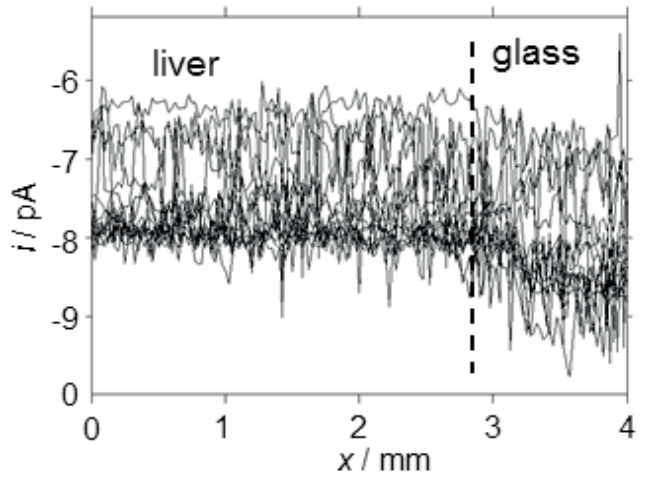

Fig.6.6 Line scans over different locations in mouse liver in phosphate buffer without the presence of GONR. a) $E=0.4 \mathrm{~V}$. b) $E=-0.4 \mathrm{~V}$. Experimental conditions: phosphate buffer 10 $\mathrm{mM}, \mathrm{pH} 6.0, \mathrm{Ag}-\mathrm{QRE}$, step size $=25 \mu \mathrm{m}$, translation speed $50 \mu \mathrm{m} / \mathrm{s}$.

Furthermore, it is important to understand the degree of redox mediator accumulation inside the biological material and the possible time dependence of such process. For this, the samples used in Fig.6.7 were separately immersed in $2 \mathrm{mM}$ solutions of FcMeOH and $\left[\mathrm{Ru}\left(\mathrm{NH}_{3}\right)\right]_{6}{ }^{3+}$, respectively. After 2 hours, the redox mediator solutions were removed and the samples quickly immersed and imaged by SECM in pure $\mathrm{PB}$. This concept is based on the detection of higher currents over the tissue in case of prior accumulation of the redox mediator diffusing from the tissue into the redox mediator-free PB solution being then detected by the SECM mode in a sample generation collection equivalent mode. In fact, Fig.6.7a shows that a certain amount of $\mathrm{FcMeOH}$ was accumulated in the tissues as an increased current was measured during the first two line scans. After few line scans, FcMeOH was highly diluted into the solution and no longer detectable. On the contrary, a significant current was observed for $\left[\mathrm{Ru}\left(\mathrm{NH}_{3}\right)_{6}\right]^{3+}$ during all line scans (Fig.6.7b). This could be caused by $\left[\mathrm{Ru}\left(\mathrm{NH}_{3}\right)_{6}\right]^{3+}$ either accumulating more than $\mathrm{FcMeOH}$ or being faster diffusing back into the solution increasing the redox mediator concentration in the buffer solution (higher currents even 
over glass). These results suggest that redox mediators could accumulate in the biological material and cause certain effects, such as higher background signal, but the real contribution during the measurements in redox mediator containing solution remains unclear. However, because the PEG-MNP-GONRs are accumulated in a threedimensional organization in the tissue redox mediator permeation into the tissue could have an advantage leading possibly to signal amplification.

Considering both the possible influences of redox active proteins and redox mediator accumulation, the regeneration currents when measuring the tissues were higher with $\mathrm{FcMeOH}$ than with $\left[\mathrm{Ru}\left(\mathrm{NH}_{3}\right)_{6}\right]^{3+}$, even in presence of PEG-MNP-GONRs. This was different for the MNP-GONR-coated PVDF membranes, which were free of biological material. However, the currents over the tissue areas were remarkably homogeneous for all analyzed tissues.

a) PB, 0.4V

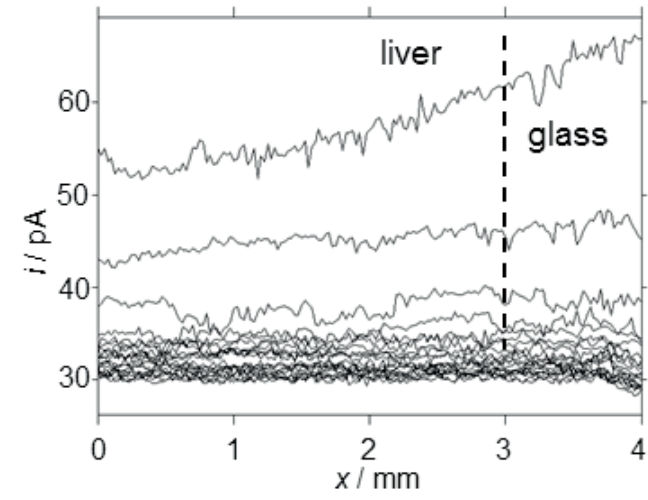

b) $\mathrm{PB},-0.4 \mathrm{~V}$

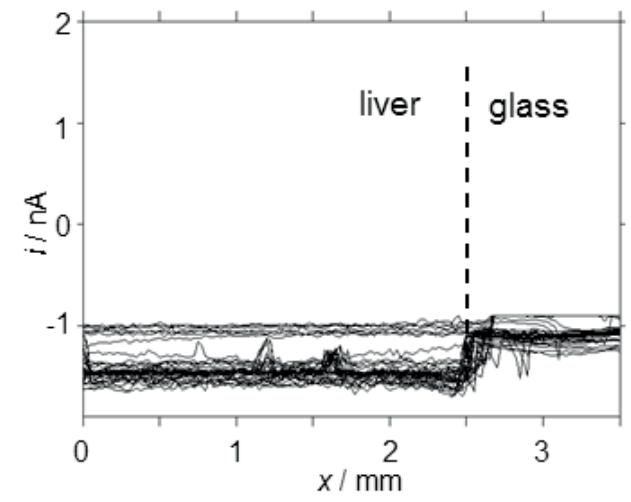

Fig.6.7 Line scans over different locations in mouse liver in phosphate buffer without the presence of GONR. a) sample immersed in $\mathrm{FcMeOH}$ and followed by $\mathrm{PB}$. Applied potential $=$ 0.4 V. b) sample immersed in $\left[\mathrm{Ru}\left(\mathrm{NH}_{3}\right)_{6}\right]^{3+}$ and followed by PB. Applied potential $=-0.4 \mathrm{~V}$. Experimental conditions: phosphate buffer $10 \mathrm{mM}, \mathrm{pH} 6.0 \mathrm{Ag}-\mathrm{QRE}$, step size $=25 \mu \mathrm{m}$, translation speed $50 \mu \mathrm{m} / \mathrm{s}$.
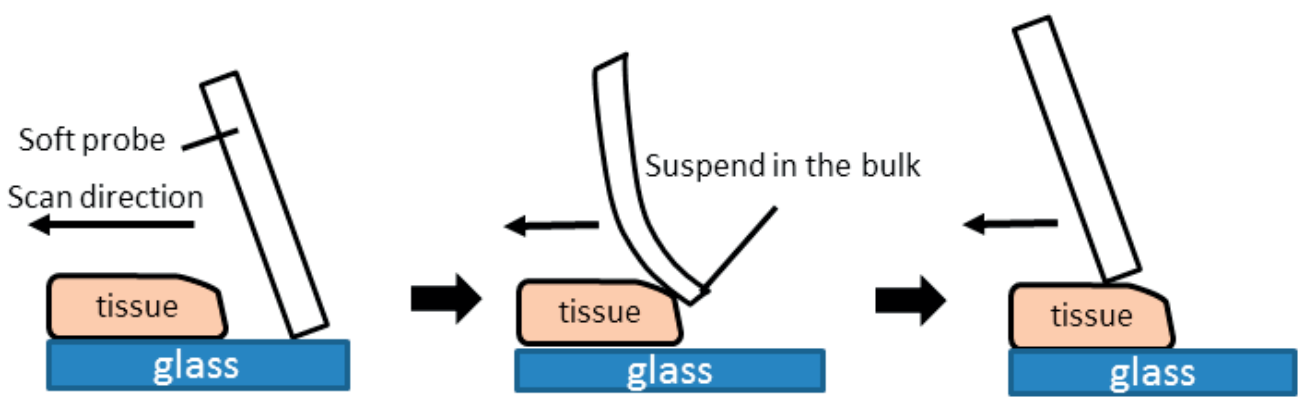
Fig.6.8 Schematic representation of the soft probe scanning over the glass and the tissue.

A closer look into the SECM image of Fig.6.5b where FcMeOH was used as redox mediator for PEG-MNP-GONR-treated tissues reveals clearly hexagonal microstructures in the tissue, which correspond to hepatic lobules in the liver as shown schematically in Fig.6.9a. A lobule functions as a small filtration unit that processes the blood coming from a central vein and releases the resulting blood through an outgoing hepatic venule. ${ }^{[247]}$ The hexagonal microstructures in the SECM image show diameters around $0.5 \mathrm{~mm}$, which correspond to the values obtained from microscopic investigations reported in literature. ${ }^{[248]}$ The observed increased feedback currents over the lobule suggest that the PEG-MNP-GONR nanomaterials were transported through the central vein and metabolized through the RES (Fig.6.9b).

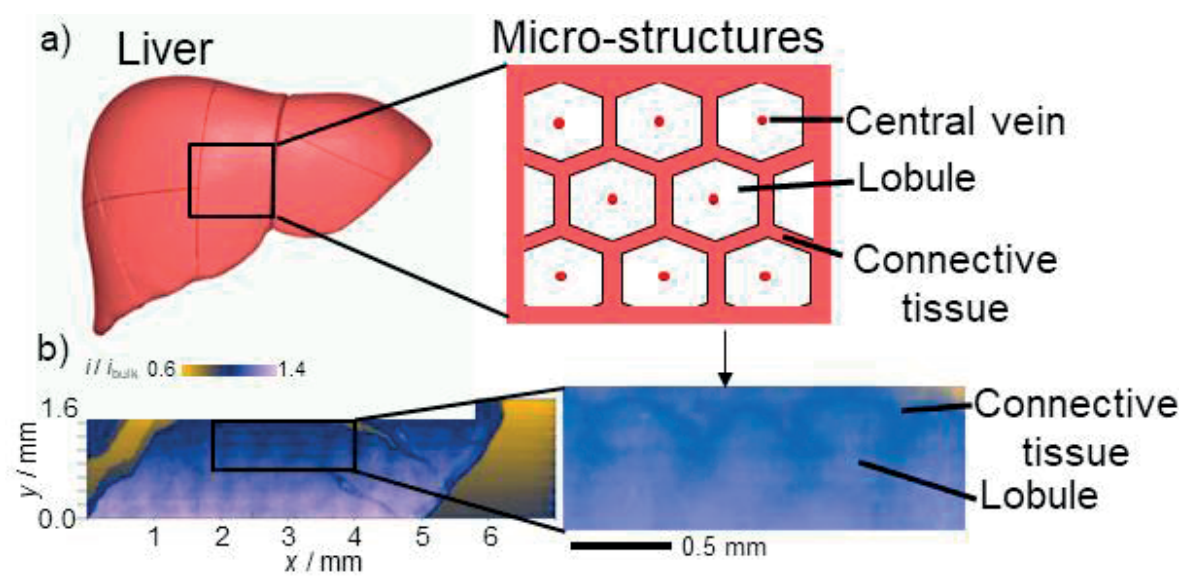

Fig.6.9 a) Liver is composed of hexagonal micro-structures called lobule. b) Zoom in the Fig.6.5b and adjust the color code and contrast, the structure of lobule can be revealed.

For comparison, whole-body autoradiography (Fig.6.10a-b) and SPEC/CT (Fig.6.10c) analyses of a cryo-sectioned mouse with in vivo injected ${ }^{99 \mathrm{~m}} \mathrm{Tc}$-labeled PEG-MNP-GONRs were performed. In the autoradiography pictures, it can be seen clearly that the PEG-MNP-GONRs accumulated mostly in the liver. In the SPECT/CT images, a similar biodistribution of ${ }^{99}$ Tc-labeled PEG-MNP-GONRs $2 \mathrm{~h}$ after injection was revealed but also showed a strong signal in bladder, which can be seen in the crosssection for the autoradiography. The detection of the PEG-MNP-GONRs in bladder shows that the excretion process has already been started, which is an important result considering that the nanoparticles, e.g. when used as drug carriers, must be removed from the body. In comparison with the two traditional bioimaging methods, SECM 
imaging can provide detailed information about the localization of the accumulated nanomaterials in the liver cross-sections. Although whole-body autoradiography analysis and SPECT/CT images can provide the information of the macroscopic distribution of the nanomaterials, the more resolved details up to micrometer scale are missing. In addition, radiolabels have certain life time for the analysis, while SECM of conductive materials can be done at any time after the mice experiment. Autoradiography can take hours to days. Soft-Probe-SECM imaging with microelectrode arrays can scan such areas in equal or shorter times (depending on the number of microelectrodes). Therefore, SECM can be a good auxiliary for visualizing the micro-biodistribution of nanomaterials in tissue microstructures.

a)

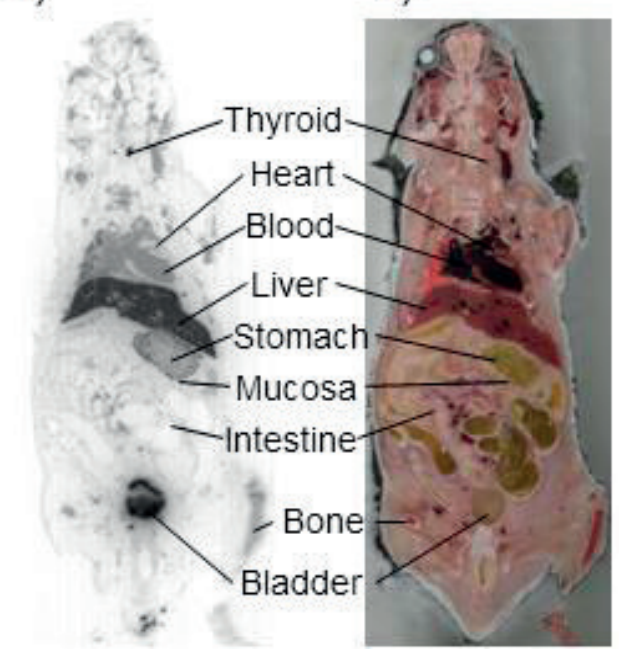

c)

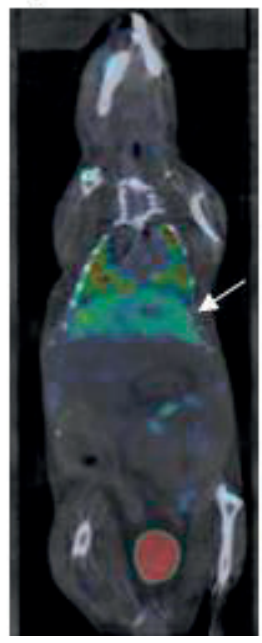

Fig.6.10 a) Whole-body autoradiography image of ${ }^{99 \mathrm{~m}} \mathrm{Tc}$-labeled PEG-MNP-GONRs complex obtained $2 \mathrm{~h}$ after injection. b) The photograph of the mouse section for whole-body autoradiography. The liver is indicated by the black arrow in (a) and (b). c) SPECT/CT image of the ${ }^{99 \mathrm{~m}}$ Tc-labeled PEG-MNP-GONRs complex in mouse. The liver is pointed out by the white arrow. Red and green colors mean very high and high concentration of radio labels. Two different mice were analyzed in (a,b) and (c). This figure is kindly provided by Dr. Yu-Ren Lu.

Compared to thin tissue sections, much larger topographic features up to millimeters need to be considered for the investigation of thick tissues with the thickness of 1-3 mm. The challenges for SECM probes to analyze such samples are much bigger, because thick tissues can be very rough and soft with the ability to swell when immersed for longer times in solutions. In order to scan such samples with SoftProbe-SECM, the stair case shaped soft probe was used. The details of the probes were 
introduced in the previous chapter. Fig.6.11 displays the SECM images of two thick liver tissues from two mice from which one was exposed to prior in vivo PEG-MNPGONR injection while the other one was not. A tiny soft probe tip (width $<100 \mu \mathrm{m}$ ) was applied scanned with stair case shaped probe. After the SECM image, no damages of the tissues were identified thanks to the softness of the SECM contact mode probe. In agreement with the results obtained on thin tissue sections, the SECM feedback current over the thick tissues treated with PEG-MNP-GONR was significantly higher than for the untreated thick tissues. However, also for these tissue samples, it must be noted that redox mediator permeability and sample porosity could contribute to the current signals. Also surface features of the size of the tip could cause minor effects on the scanning. However, since a specific marker is analyzed on two samples of identical structure, significant differences in the measured signals can be correlated to the accumulation or absence of the injected nanoparticles. Compared with the thin tissue sections, the morphology of thick tissue sections is much closer to those of real animal or human organs demonstrating the suitability of soft probes for a broad range of biological applications. 


\section{a)}
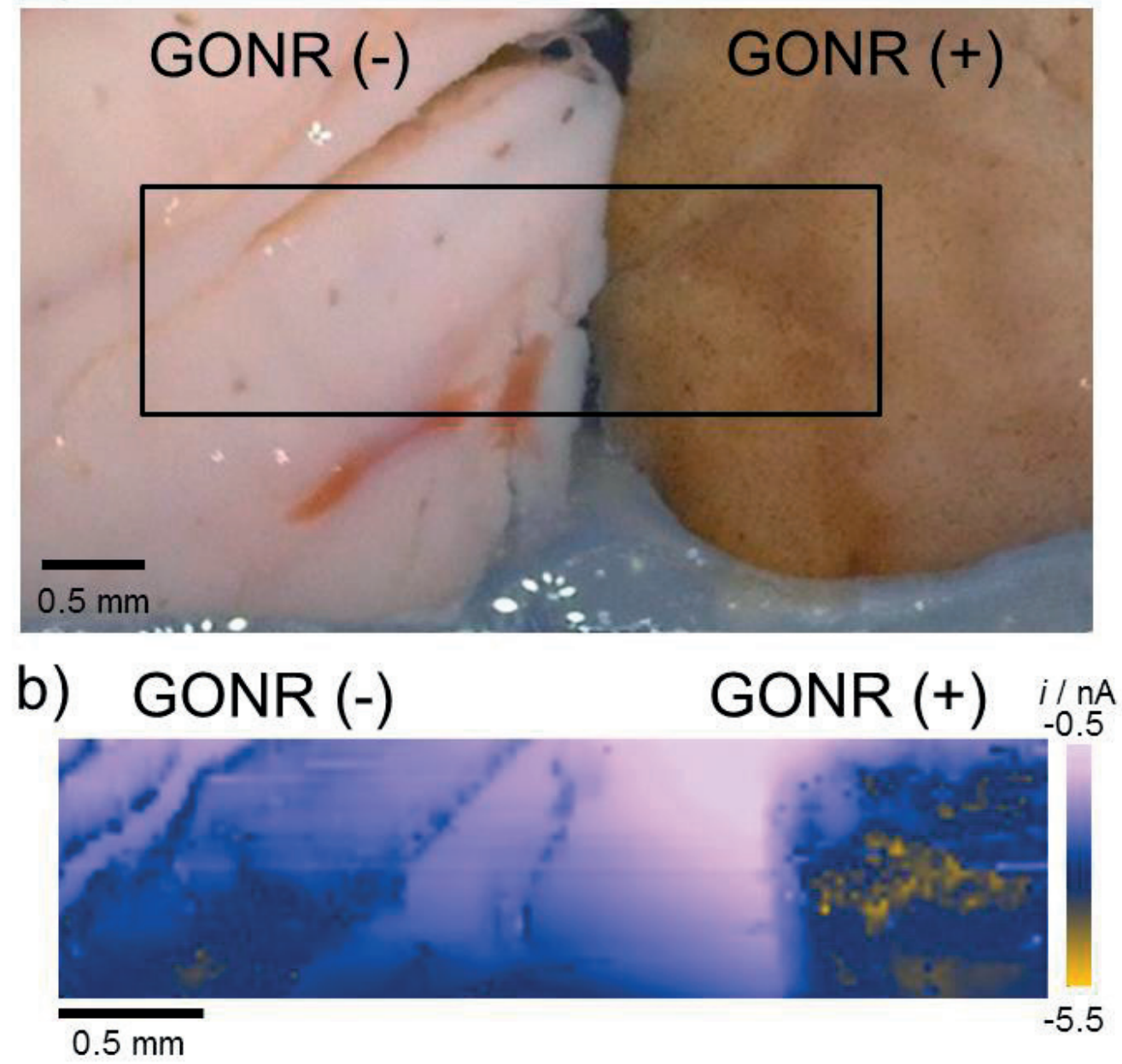

Fig.6.11 a) Optical picture of two mice livers. The area scanned by Soft-Probe-SECM is marked with a black rectangle. b) SECM image of the two mice livers in the presence and absence of PEG-MNP-GONR injection scanned in contact mode by soft probe. Experimental conditions: $2 \mathrm{mM}\left[\mathrm{Ru}\left(\mathrm{NH}_{3}\right)_{6}\right]^{3+}$ and phosphate buffer $10 \mathrm{mM}, \mathrm{pH}$ 6.0Ag-QRE, step size $=25 \mu \mathrm{m}$, translation speed $50 \mu \mathrm{m} / \mathrm{s}, E=-0.4 \mathrm{~V}$.

\subsection{Conclusion}

To conclude, a strategy for the detection of PEG-MNP-GONR composites in mouse liver tissues by using Soft-Probe-SECM was developed. Feedback mode imaging of large areas using ferrocene methanol and ruthenium hexaammine as redox mediators resulted in PEG-MNP-GONR maps of micrometer resolution. The PEGMNP-GONRs were found in the lobule in the liver but less in connective tissue. In 
comparison to standard bio-imaging techniques, such as autoradiography and SPECT/CT, SECM does not require radiolabels and can compete in terms of resolution. Furthermore, the application of soft probes does not only enable the analysis of thin but also of thick tissue sections thanks to the contact mode scanning. Soft-Probe-SECM is an electrochemical tool with high potential for biological applications. Nevertheless, the complexity of biological samples, such as the presence of other redox active centers, such as proteins, the permeability of the redox mediator and the porosity of the samples, needs to be considered and are part of our further studies. 


\section{Mapping the antioxidant activity of apple peels with soft probe scanning electrochemical microscopy}

Adapted from: Tzu-En Lin, Andreas Lesch, Chi-Lin Li, Hubert H. Girault Journal of Electroanalytical Chemistry, 2017, 786, 120 - 128.

\subsection{Introduction}

Antioxidants (AOs) are well-known beneficial dietaries for reducing the risks of cardiovascular disease and cancer. ${ }^{[249,250]}$ They also protect fruits from oxidative stress caused by unfavorable external environments such as drought, chilling injury, UV radiation or pathogen attack. ${ }^{[251-254]}$ Hence, AOs in fruits are generally more concentrated in the peel than in the flesh representing a major fruit protection barrier. ${ }^{[255,256]}$ Several enzymatically assisted mechanisms provide a certain degree of regeneration of AOs after the AOs degradation by free radicals. Consequently, the AO concentration in the peel is a quantitative indicator for the status of the AO defense system of the fruit.

Apples have a high content of AOs making them highly valuable crops from which thousands of tons are consumed worldwide every day. Therefore, the understanding and support of their AO defense system is an important issue for apple cultivators. ${ }^{[257,258]}$ The AO defense system of apples is rather complex and depends on many factors, such as apple variety and growing as well as storage conditions. It has been reported that polyphenolic compounds, in particular flavanols (e.g. catechin and procyanidin), flavonoids (e.g. quercetin) and hydroxycinnamic acid derivates (e.g. chlorogenic acid), show the highest AO activity. ${ }^{[252,253,259]}$ Other AO molecules, such as glutathione (GSH) or ascorbate (vitamin C, L-AA), participate in the removal of free radicals. ${ }^{[260]}$ Although L-AA is up to six times more concentrated in the apple peel than in the fruit part, the contribution of L-AA to the overall AO activity is rather small. ${ }^{[257]}$ Antioxidative enzymes, such as glutathione reductase, ascorbate peroxidase and catalase, are involved in reducing free radicals and $\mathrm{H}_{2} \mathrm{O}_{2}$, while they also keep the level of GSH and L-AA at 
sufficient levels within the glutathione-ascorbate cycle. ${ }^{[261]}$ The major part of these AOs is fixed in the apple matrix as glycosides and carboxylic acid esters, ${ }^{[262,263]}$ which usually requires vigorous extraction and purification methods such as HPLC followed by the quantification with optical detection methods. ${ }^{[264,265]}$ The sample preparation and optical measurement procedures are time consuming and optical methods can be interfered by sample color background (color pigments in fruits) or autofluorescence. ${ }^{[266-268]}$ Furthermore, although the peel and flesh of the apple can be separated prior to the extraction and $\mathrm{AO}$ quantification, spatial information about the local distribution of AOs, for example over the peel surface, cannot be obtained. In addition, once an apple is prepared for analysis using the invasive methods described above, the $\mathrm{AO}$ content of this particular fruit cannot be followed further requiring the investigation of statistically relevant amounts of apples for aging studies.

Therefore, developing simple and non-destructive sensors for analyzing rapidly, at low cost and on site the status of the AO defense system with high spatial resolution is attractive for apple growers. UV sensitive AOs in the outer layer of the apple peel have recently been measured directly and non-invasively on the fruit by using reflectance and chlorophyll fluorescence spectroscopy. ${ }^{[269-271]}$ However, these approaches can only be applied to optically active compounds. Alternatively, AOs can be detected at amperometric electrodes where the AOs get electrochemically oxidized or reduced depending on the applied electrode potential. ${ }^{[272]}$ The recorded current is proportional to the concentration of AOs in the sample and common optical limitations (e.g. interferences from colorful pigments) do not occur. For this analysis, the AOs need to be extracted from the apple and dissolved in an electrolyte solution. Nevertheless, the electrochemical detection of certain AOs can suffer from slow kinetics and/or interferences by other electrochemically active compounds. One approach is to use redox mediators that, for instance, get reduced by the AOs and then electrochemically oxidized at the electrode resulting in a redox mediator recycling. ${ }^{[273]}$ The standard redox potential of the AOs must be lower than the potential of the redox mediator. These redox active compounds are generally embedded in the matrix of chemically modified electrodes but can also be present in solution. ${ }^{[274-276]}$ The latter concept is related to the well-known ferric ion reducing antioxidant power (FRAP) assay where $\mathrm{Fe}^{3+}$ ions are reduced by $\mathrm{AOs}$ to $\mathrm{Fe}^{2+}$ ions to determine the total AO's reducing capacity by a detectable color change of the iron complex. ${ }^{[277]}$ 
Conventionally, SECM is operated in constant height mode requiring flat and nontilted substrates. Natural samples such as apples contain curved topographic features that are reasonably larger than the probe dimension. This leads to misleading information caused by the varying working distance between the probe and the sample. In this work, we employed a soft carbon-based SECM probes ${ }^{[12]}$ as ideal scanners or measurements on the fragile and curved peel of apples (Scheme 7.1). Ferrocene methanol $(\mathrm{FcMeOH})$ was used as redox mediator that was electrochemically oxidized under diffusion control to $\mathrm{FcMeOH}^{+}$at the scanning microelectrode. The result is a steady-state current. When the probe is brought in close proximity to the sample, $\mathrm{FcMeOH}^{+}$reaches by diffusion the apple peel where the reaction of $\mathrm{FcMeOH}^{+}$with the AOs inside the apple peel regenerates FcMeOH (known as SECM feedback mode). This additional flux of $\mathrm{FcMeOH}$ towards the microelectrode results in an increased current signal (i.e. "regeneration current") compared to a lower current over purely insulating substrates where no regeneration takes place and just hindered diffusion of the redox mediator is observed. In this way, the AO activity initiated by molecular and enzymatic systems in the apple peel and able to reduce $\mathrm{FcMeOH}^{+}$was mapped. Similarly, FcMeOH had been used previously by other groups in SECM related approaches to detect the status of the AO molecule GSH in cancer cells. ${ }^{[176,278]}$ Furthermore, an apple peel sample was exposed to hydrogen peroxide solution to degrade artificially the AO activity as clearly visualized by subsequent SECM imaging. Finally, the AO activity and the gradient of AO concentration in an apple cross-section were mapped.

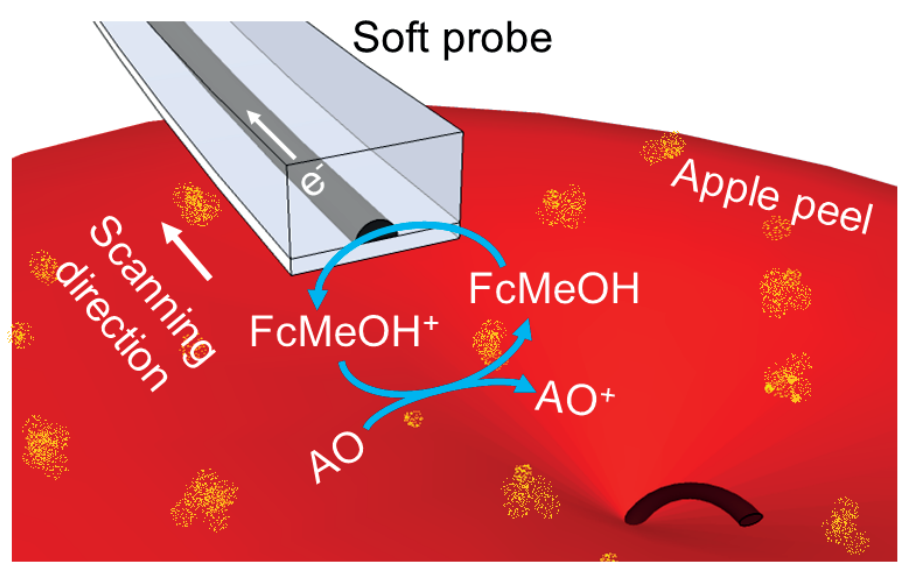

Scheme 7.1 Schematic representation of a soft probe detecting AOs on a curved apple surface by the regeneration current of $\mathrm{FcMeOH}$ caused by AOs present in the apple peel. 


\subsection{Results and discussions}

When the carbon microelectrode is positioned in the bulk solution (i.e. $\left.h_{\mathrm{P}}>>0\right)$, a diffusion-limited steady-state current $i_{T, \infty}$ for the electrochemical oxidation of $\mathrm{FcMeOH}$ is recorded. While the probe is approaching an insulating glass surface the diffusion of $\mathrm{FcMeOH}$ gets physically hindered by the glass substrate and the recorded tip current $i_{\mathrm{T}}$ decreases (red curve in Fig. 0a with normalized current $i_{\mathrm{T}} / i_{\mathrm{T}, \infty}$ ) until the soft probe touches the glass surface $\left(h_{\mathrm{P}}=0\right)$. At this point the soft probe starts sliding forward over the glass surface while the working distance $d$ is kept constant resulting in a barely changing current. The remaining current is a result of the diffusion of $\mathrm{FcMeOH}$ from the bulk solution around the soft probe tip. When approaching a smooth part of an apple peel with the same soft probe and within the same electrochemical cell (black curve in Fig. 7.1a), the measured tip current decreases less strongly and is larger at physical contact with the apple peel compared to the theoretical negative feedback current for a totally inactive substrate such as glass. This is an indication for redox mediator regeneration induced by the apple peel AOs. Indeed, the recorded regeneration current is smaller than the diffusion-controlled oxidation current of $\mathrm{FcMeOH}$ in bulk solution demonstrating finite kinetics of the regeneration reaction, a small amount of accessible active $\mathrm{AO}$ centers and/or a slow mass transport of $\mathrm{FcMeOH}$ through the various layers of the apple peel matrix with likely different porosity. Besides these three effects, the microscopic apple peel topography could influence the measured current signal by influencing the diffusion of the redox mediator. A varying sample tilt shows a limited influence on the recorded current as demonstrated previously. ${ }^{[71]}$ Furthermore, organic compounds, which are electrochemically inactive in the applied electrochemical window, could slowly diffuse out from the apple peel and contaminate the electrode surface. Consequently, the absolute current measured at the electrode goes down (vide infra), whereas electrode fouling was not observed when measuring the glass sample. 


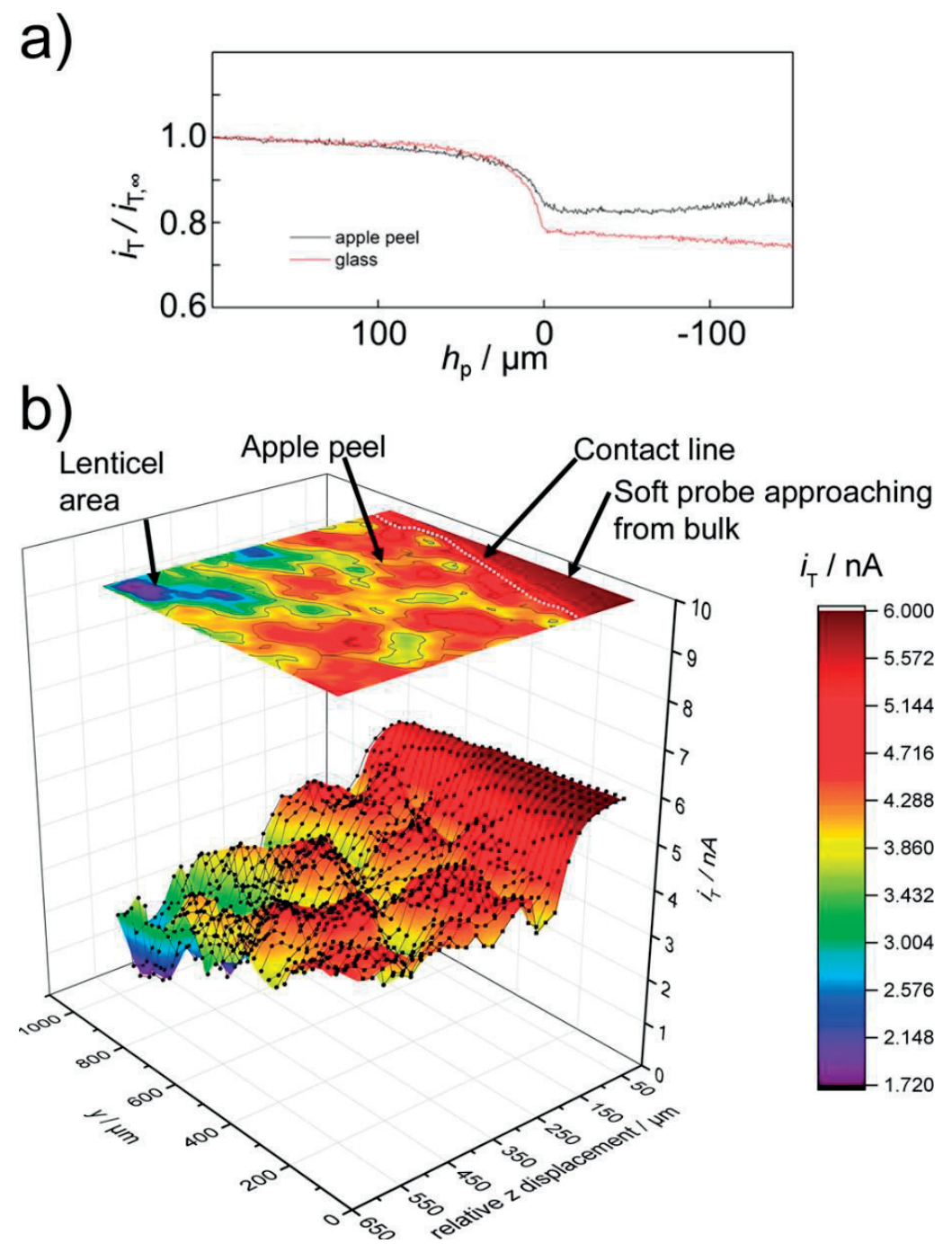

Fig. 7.1 a) Feedback mode approach curves over a smooth apple peel and flat glass substrate. b) Series of adjacent approach curves on an apple peel. Upon mechanical contact the soft probe slides forward over the apple as a result of the continued vertical approaching of the probe holder. Experimental conditions: $E_{\mathrm{T}}=0.4 \mathrm{~V}$, vertical step size $=20 \mu \mathrm{m}$, horizontal step size $=$ $25 \mu \mathrm{m}$, translation speed $=50 \mu \mathrm{m} / \mathrm{s}$. The experimental solution contained $2 \mathrm{mM} \mathrm{FcMeOH}$ in $10 \mathrm{mM}$ phosphate buffer (PB), pH 6.

Generally, the reaction of free radicals with AOs is very fast. However, most AOs are embedded inside the apple peel and the exact transport mechanism of FcMeOH into the apple peel compartments is unknown yet. It is known that an intracuticular lipophilic wax is present within a more hydrophilic cutin polymer matrix in the cuticular layer of the apple peel forming a water-in-oil emulsion like structure. ${ }^{[126]}$ This layer contains certain amounts of the hydrophilic and lipophilic AOs (vide supra). ${ }^{[26]}$ Because $\mathrm{FcMeOH}$ shows neither unequivocal hydrophilic nor hydrophobic 
characteristics, it was chosen as the redox mediator in this study. Furthermore, SECM approach curves, such as those shown over the glass and the apple peel, do theoretically not cross each other, because the shape of such curves is mainly dependent on the substrate kinetics. The observed crossing in Fig. 7.1a is most likely a result of the macroscopic apple topography that affects the diffusion of $\mathrm{FcMeOH}$ and influences the tip current as long as the soft probe is not in contact with the substrate.

The validity of the feedback mode concept was supported by cyclic voltammetry in solutions containing various concentrations of the AOs quercetin and GSH. The AOs caused a clear amplification of the steady-state current for the oxidation of $\mathrm{FcMeOH}$ (Fig. 7. 2). ${ }^{[279]}$ The CVs of GSH and quercetin in absence and presence of FcMeOH are presented in Fig. 7. 2. In the solutions, which did not contain FcMeOH (Fig. 7. 2a and c), the current signals were very low. However, in the presence $2 \mathrm{mM} \mathrm{FcMeOH}$, the current signals increase with $\mathrm{AO}$ concentration indicating the recycling of $\mathrm{FcMeOH}$ by AOs (Fig. 7. 2b and d).

a)

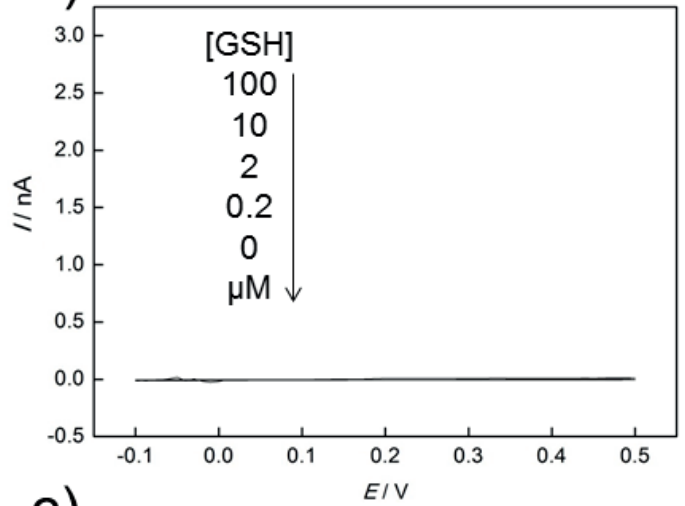

c)

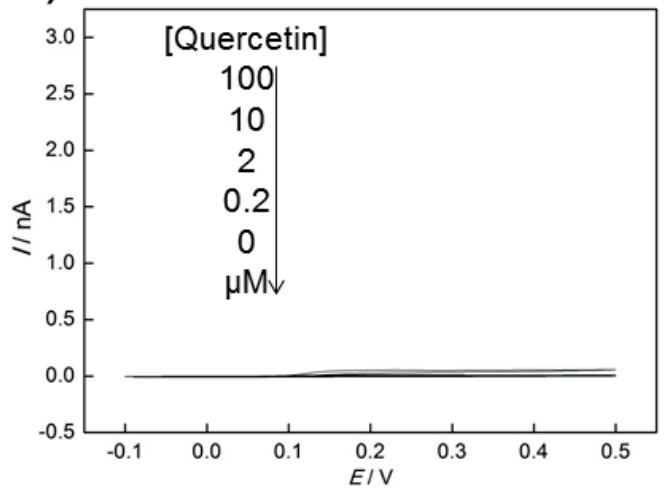

b)

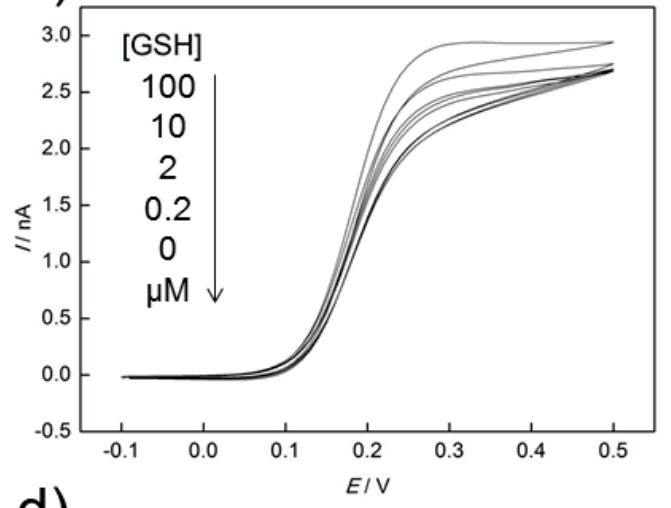

d)

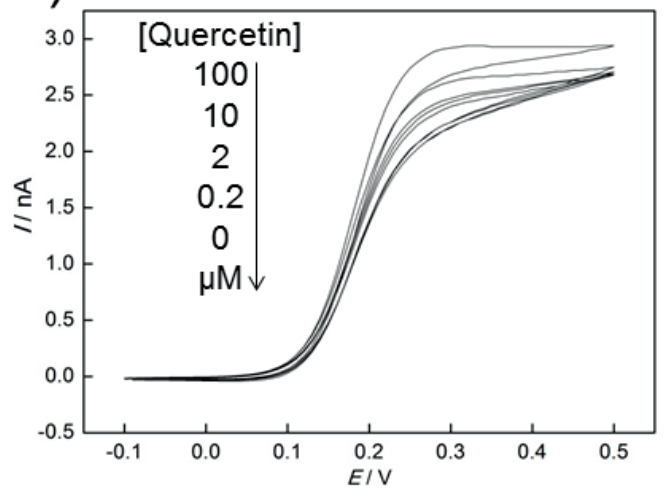

Fig. 7.2 a) CVs of various concentrations of GSH in $10 \mathrm{mM}$ PB. b) CVs of GSH in the presence of $\mathrm{FcMeOH}$. c) CVs of quercetin in $10 \mathrm{mM}$ PB. d) CVs of quercetin in the presence of FcMeOH. 
Experimental conditions: $2 \mathrm{mM} \mathrm{FcMeOH}$ in $\mathrm{PB}(\mathrm{pH} 6,10 \mathrm{mM})$. Scan rate $=10 \mathrm{mV} / \mathrm{s} . \mathrm{WE}=$ Soft stylus probe. $\mathrm{RE}=\mathrm{Ag} / \mathrm{AgCl} . \mathrm{CE}=\mathrm{Pt}$.

In order to demonstrate the stability and reproducibility of approach curves along such rough and delicate surfaces, a series of parallel approach curves on the apple peel was performed and the recorded tip current was plotted as a function of the horizontal and vertical probe position (Fig. 7.1b). Once the soft probe got in contact with the sample and started a horizontal probe movement on the apple peel, the recorded approach curve experiments turned into a 2D mapping of the substrate reactivity. Such combined approach curve-imaging experiment demonstrated the stability of the probe sliding as well as a stable electrochemical signal. While sliding over the apple peel surface, regions of higher (yellow-red in Fig. 7.1b) and lower (blue) redox mediator regeneration capabilities were identified. The origin of these signals is a non-uniform apple peel surface that contains specific microstructures of which some appear for instance as yellow spots (Fig. 7.3a). 

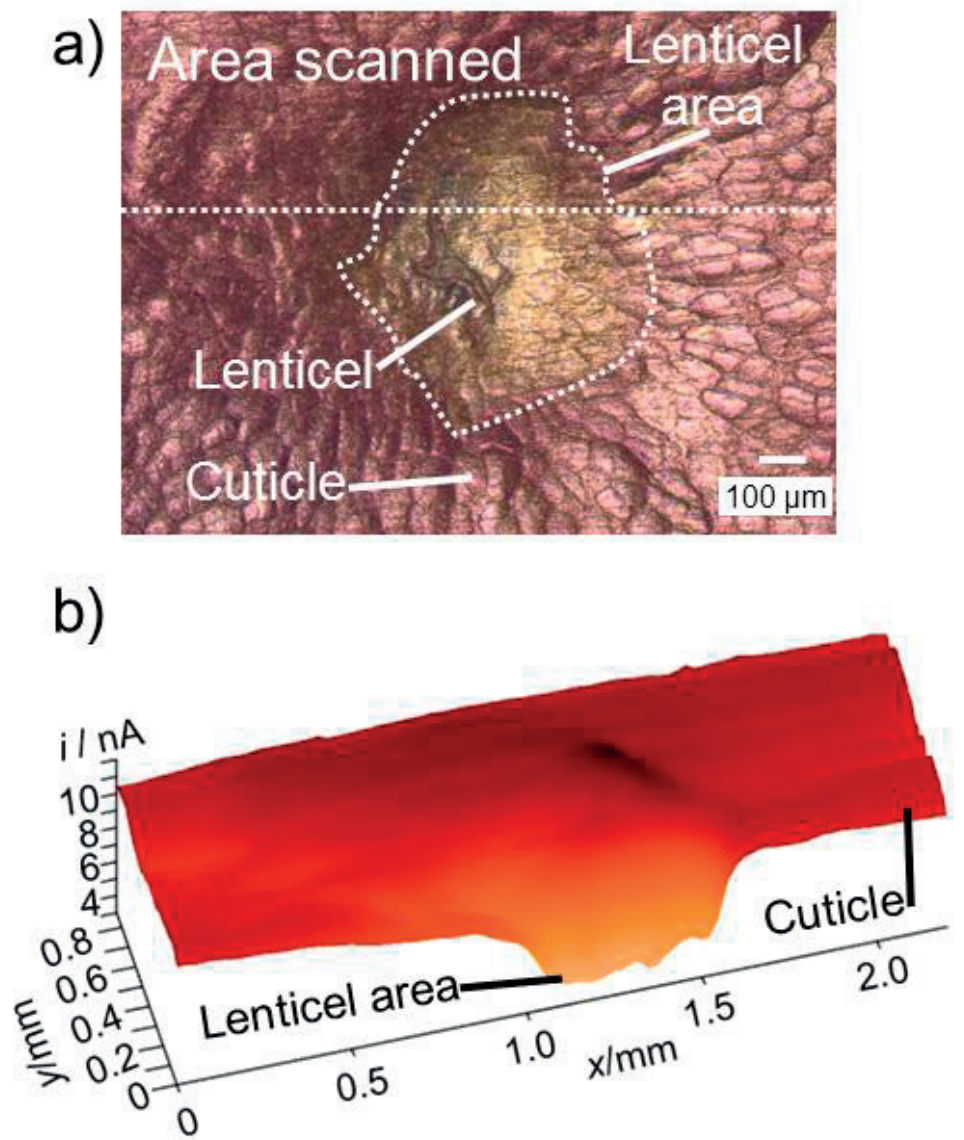

c)

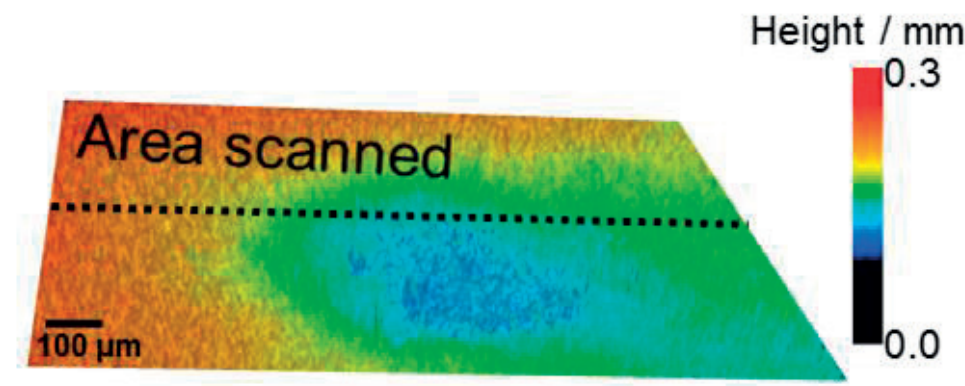

Fig. 7.3 a) Laser optical microscopy image of lenticel region on an apple peel. The area scanned by SECM is located above the white dashed line. b) Soft-Probe-SECM image of lenticel area on an apple peel. c) Topography of the studied yellow spot on the apple peel. The scanned area is located behind the black dashed line. Experimental details: working potential $E_{\mathrm{T}}=0.4 \mathrm{~V}$, probe translation speed $=50 \mu \mathrm{m} / \mathrm{s}$, step size $=25 \mu \mathrm{m}, h_{\mathrm{P}}=-200 \mu \mathrm{m}$ and delay time between probe movement and current reading $=0.1 \mathrm{~s}, 2 \mathrm{mM} \mathrm{FcMeOH}$ in $10 \mathrm{mM}$ phosphate buffer (PB), pH 6.

These areas consist of special cells called lenticels (Fig. 7.4), which are porous tissues with large intercellular spaces located in the periderm of fruit peels. The lenticels 
are formed from cracks that arise when the fruit peel expands during apple growing and can act as pores for the interchange of gases between the interior tissue and the surrounding air. The AO defense system of a fruit starts a mechanism to accumulate AOs, mainly phenolic compounds, around such lenticel to protect the fruit from oxidative stress induced by the air entering through this porous structure. ${ }^{[280,281]}$ As a result of this defense mechanism, the phenolic AOs located around a lenticel are mainly oxidized leading to lenticel discoloration. This can be seen as the yellowish area surrounding the lenticel, termed lenticel area in this work (Fig. 7.3a).

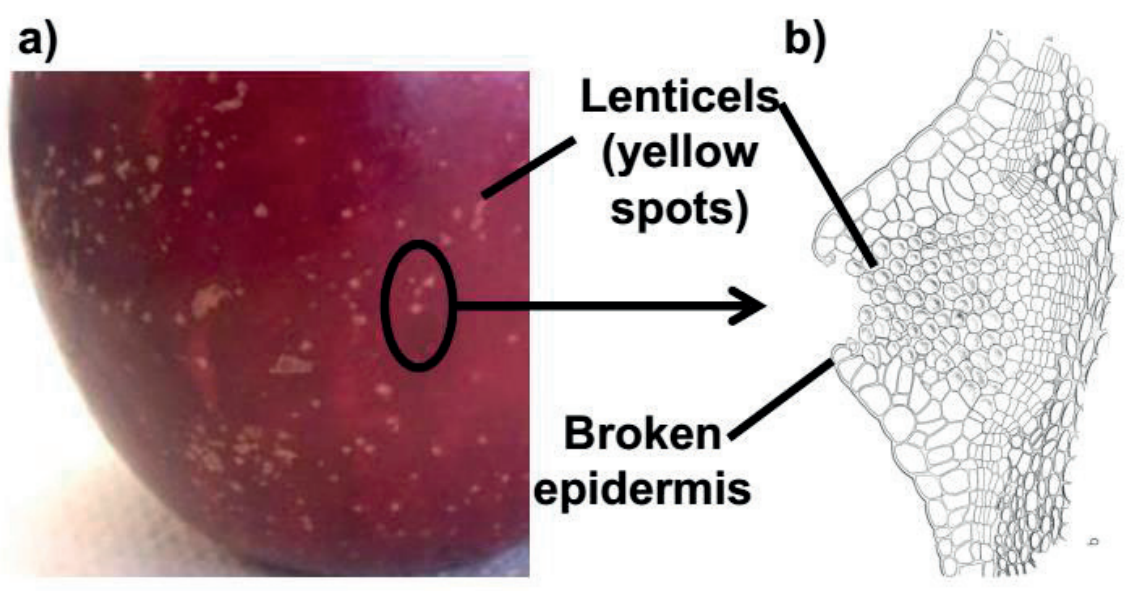

Fig. 7.4 a) Photograph of an apple with yellow spots on its peel. b) Schematic representation of a lenticel on the apple peel. These lenticels are centered in yellow spots forming a lenticel area.

A soft probe was then applied to monitor the AO activity around a lenticel on the apple peel by using the lateral SECM imaging routine where the probe was horizontally translated in a line-by-line fashion (Fig. 7.3b). The current over the lenticel and in the surrounding area was significantly lower than over the surrounding apple peel indicating the lower amount or absence of $\mathrm{AO}$ activity in this so-called lenticel area (i.e. the lenticel with periphery of reduced AO activity). It is important to note that this yellow spot area had a maximum lateral dimension of about $0.8 \mathrm{~mm}$ with a maximum depth of $100 \mu \mathrm{m}$ (Fig. 7.3c). Due to the contact mode operation, the tip of the soft probe was able to slide inside this lenticel area that was larger than probe width (i.e. $100 \mu \mathrm{m}$ ) and ensured an almost constant working distance. In fact, the probe was too wide to enter into the tiny crack of the lenticel itself $(<100 \mu \mathrm{m})$. Therefore, topographic effects over the lenticel are principally possible if the imaging resolution as achieved by the 
probe dimension and the experimental conditions is high enough. In this work, no significant topographic artifacts in the recorded current were observed over the entire lenticel area. For instance, if the probe width would be larger than the diameter of the yellow spot the probe would not enter inside the lenticels area. As a consequence, $d$ would increase over such area and result in a larger current approaching the steady-state current in bulk solution (Fig. 7.1), which was not observed. The bulk currents in CV plots (Fig. 7. 2b and d) are slightly lower than current over the lenticel area in Fig. 7.3b. This is due to the manual fabrication of different probes or different cutting that cause some slight variations in active electrode area. Therefore, the exact current values are not comparable between different SECM images.

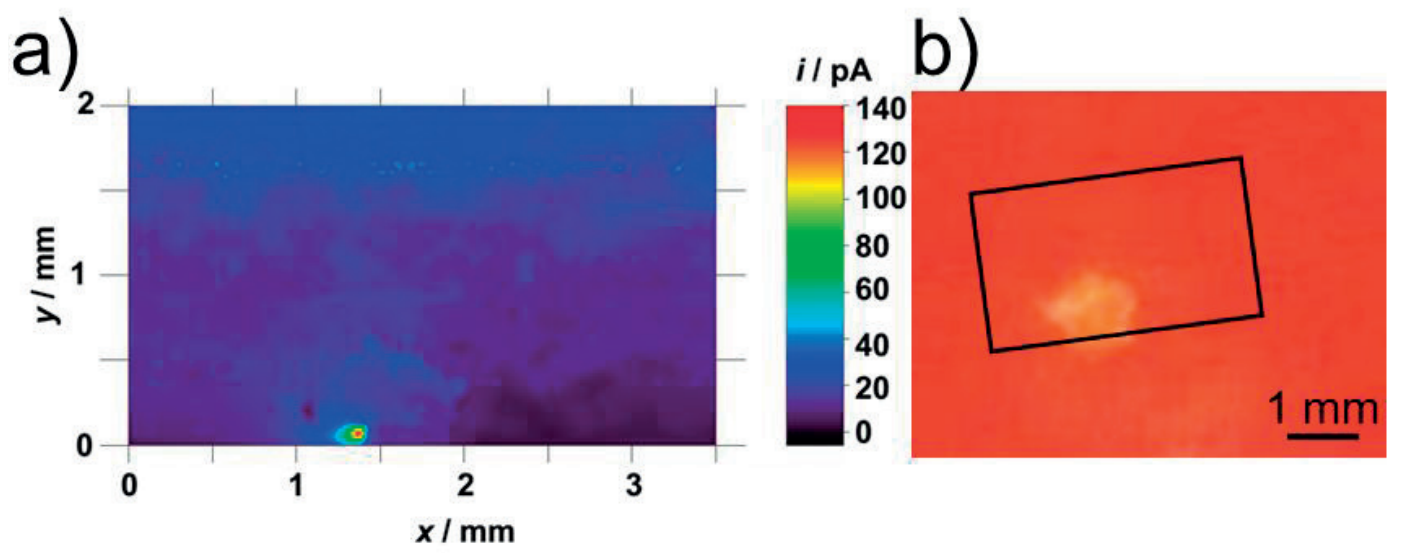

Fig. 7.5 a) Soft-Probe-SECM feedback mode image of a lenticel area. Experimental conditions: $10 \mathrm{mM}$ phosphate buffer $(\mathrm{pH}=6.0), E_{\mathrm{T}}=0.4 \mathrm{~V}, h_{P}=-200 \mu \mathrm{m}$, step size $=25 \mu \mathrm{m}$, translation speed $=50 \mu \mathrm{m} / \mathrm{s}$. b) Photograph of the apple peel sample containing the scanned as marked by the black rectangle.

The redox mediator regeneration is most likely caused by the AOs and related AO enzymatic mechanisms, which could be i) embedded inside the cuticle (redox mediator would diffuse in and out the cuticle), ii) fixed in the top layer of the cuticle (easily accessible for the redox mediator) or iii) diffusing out from the cuticle (regeneration of the redox mediator by AO molecules or direct measurement of AOs outside from the apple). The SECM imaging of the apple peel in phosphate buffer solution without redox mediator has not shown any significant current signal (Fig. 7.5). This explains that the direct electrochemical oxidation of AOs released from the apple can be excluded, but the amplification by $\mathrm{FcMeOH}$ recycling is still possible. The exact underlying 
processes are not fully understood yet.

In order to understand the permeability and the possible porosity caused by the apple peel structure, SECM imaging of the apple peel in phosphate buffer was subsequently performed without the redox mediator, (Fig. 7.6a-b), with the redox mediator (Fig. 7.6c-d), and again without the redox mediator (Fig. 7.6e-f) The recorded current in the first experiment was very low, indicating no direct signals from the AOs as expected. These data represent the baseline for the third SECM measurement in this experiment. Afterwards, $\mathrm{FcMeOH}$ solution was used and the same area was imaged again, but with more line scans. The lenticel area with lower recorded currents can be seen on the right (Fig. 7.6c-d). Afterwards, the FcMeOH solution was removed and the sample was washed twice with phosphate buffer. The same area was immediately scanned again in pure PB. (Fig. 7.6e-f). In Fig. 6c-d, the lenticel area can be seen clearly in presence of the redox mediator as presented already above. The recorded currents in Fig. 7.6e-f are significantly larger than in the first redox mediator-free experiment. This shows that $\mathrm{FcMeOH}$ had accumulated in the apple peel and diffused slowly out from apple peel surface after the solution composition was changed to the bare buffer. Especially during the first three line scans the currents were even higher. This result indicates that the $\mathrm{FcMeOH}$ diffused away from the apple peel in approximately $15 \mathrm{~min}$. This experiment indicates that the permeability of the redox mediator through the apple peel plays a role during the SECM experiment and the interpretation of the data. 

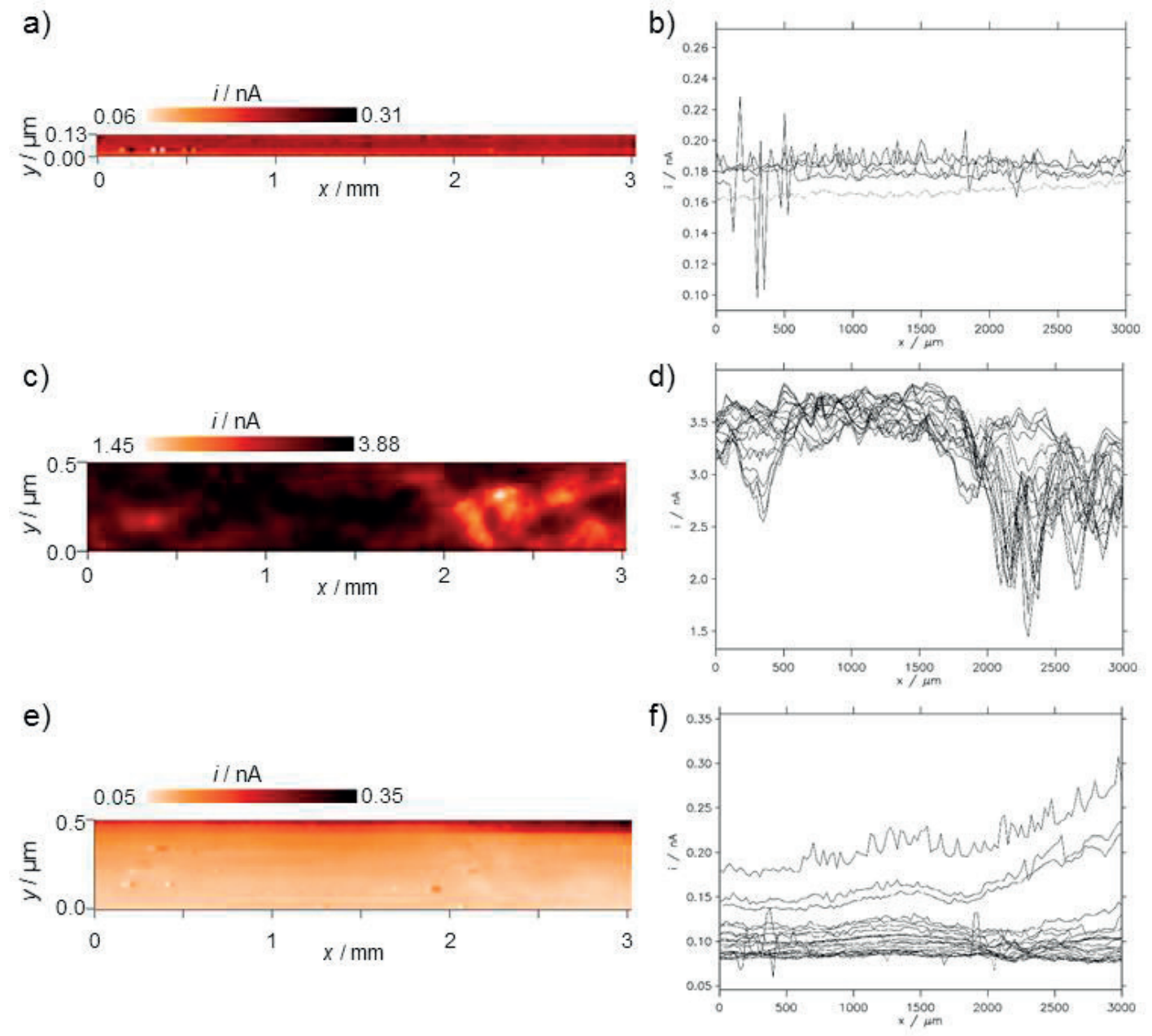

Fig. 7.6 Soft-Probe-SECM feedback mode images of an identical area of the red apple peel with lenticel area $(x=2 \sim 3 \mathrm{~mm}$ ) recorded subsequently in 1.) pure $\mathrm{PB}$ (a and b), $\mathrm{FcMeOH}$ solution (c and d) and again pure PB (e and f). For each measurement, the SECM image is shown as a map (left) and 2D plot (right). Experimental solutions: a) and c): $10 \mathrm{mM}$ phosphate buffer $(\mathrm{pH}=6.0)$. b) $2 \mathrm{mM} \mathrm{FcMeOH}$ containing $10 \mathrm{mM}$ phosphate buffer $(\mathrm{pH}=6.0)$. Other experimental conditions: $E_{\mathrm{T}}=0.4 \mathrm{~V}, h_{P}=-200 \mu \mathrm{m}$, step size $=25 \mu \mathrm{m}$, translation speed $=50$ $\mu \mathrm{m} / \mathrm{s}$. The volume of all the experimental solution were $3 \mathrm{~mL}$.

A video sequence of a soft probe sliding along the vertical cross-section of an apple peel, including a lenticel area, was recorded (i.e. a typical contact mode line scan) in order to identify a possible deformation and compression of the apple peel. In Fig. 7.7, three representative snapshots from the video are shown. The soft probe followed the topography of the apple peel and lenticel area while the peel surface seemed to get neither deformed nor compressed. However, when approaching the apple peel surface area, the soft probe slit forwards and the apple peel was slightly compressed. 
a)

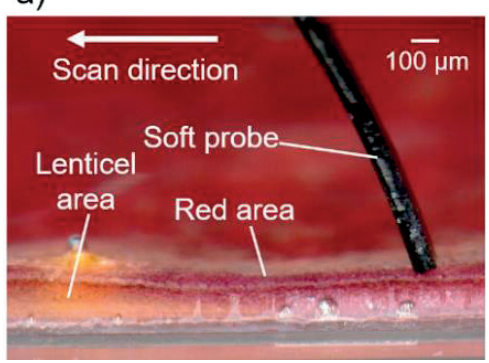

b)

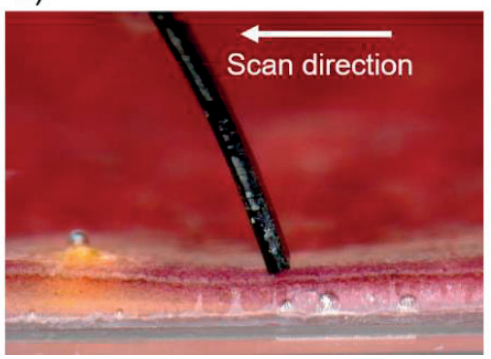

c)

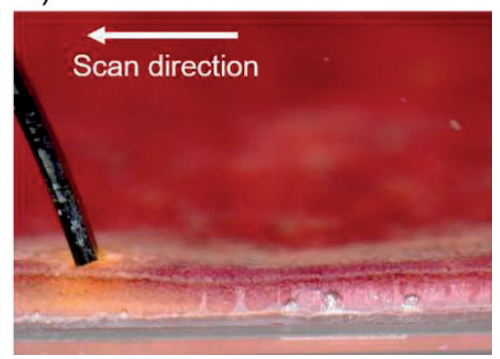

Fig. 7.7 a)-c) Optical micrographs of a soft probe line scan in contact mode on a red apple peel with lenticel area. The apple peel sample was covered with a droplet of electrolyte solution. The curved electrolyte solution-air interface resulted in a curved appearance of the straight soft probe due to the light refraction.

The videos were taken during the approaching curves, and the images extracted from the video were shown in Fig. 7.8. The upper panels of Fig. 7.8 show the probe sliding in contact mode during an approach curve on apple. A very little compression can be recognized due to a slight pressure that was not observed during line scan sliding (lower panels) where no decompression of the apple peel was recognized. This might cause the release of (electro)chemically active compounds, however, no indications for this were observed in the experiments performed (for instance, see approach curve plot in Fig. 7.1b). 

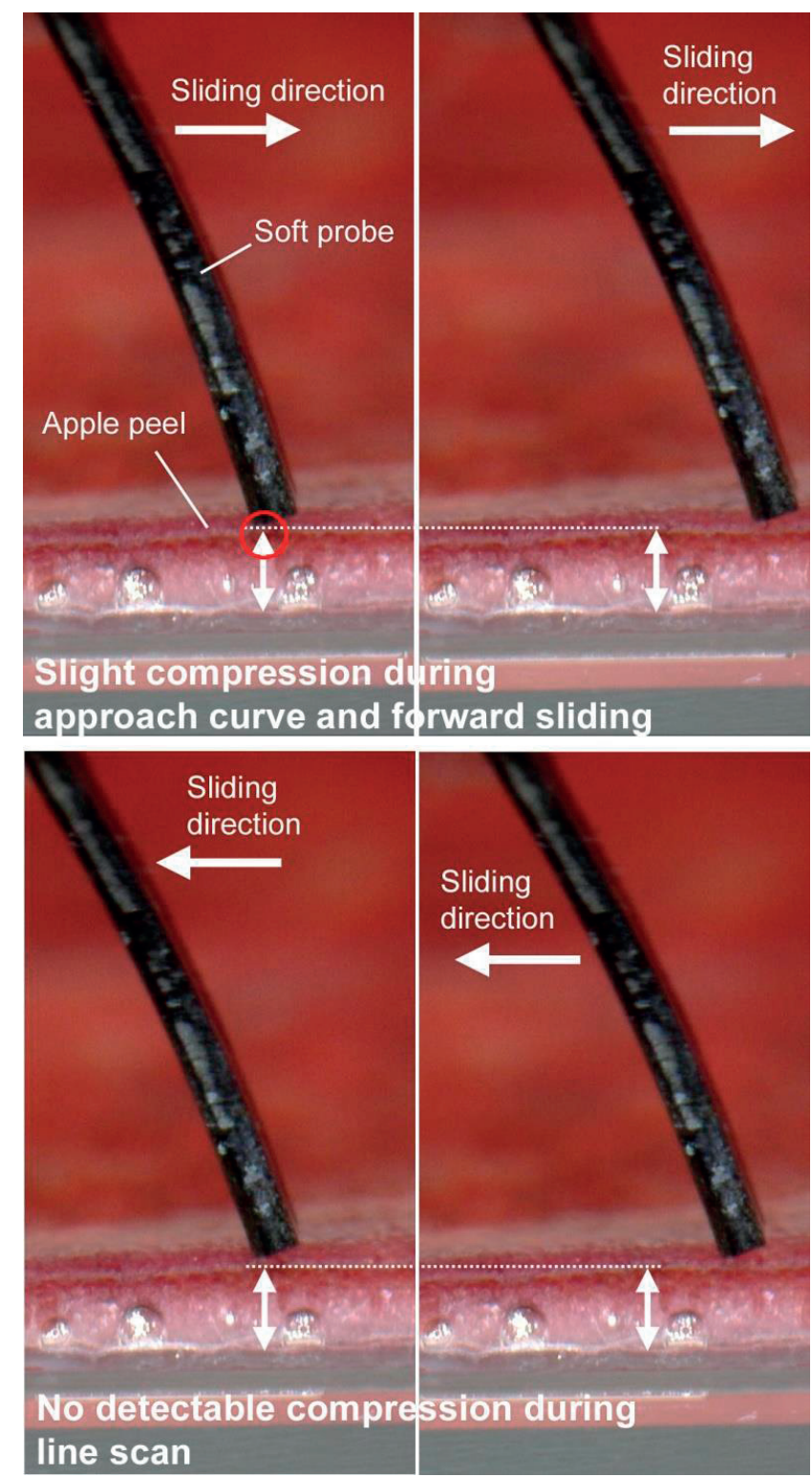

Fig. 7.8 Snapshots of recorded videos of a soft probe sliding along a vertical apple peel crosssection forward during approaching (upper panels) and during line scans (lower panels).

In order to corroborate that the distribution of the AO activity within the apple peel can be visualized with high certainty, part of the apple peel components was chemically oxidized by a controlled exposure to hydrogen peroxide $\left(\mathrm{H}_{2} \mathrm{O}_{2}\right)$ solution for $15 \mathrm{~min}$ prior to SECM imaging. Such treatment led to a strong artificial oxidative damage due to the oxidizing power of $\mathrm{H}_{2} \mathrm{O}_{2}$ for biological materials, which is directly linked to a significant degradation of the AO defense system. This might be accompanied by a change in the permeability characteristics of this peel area. The boundary between the untreated and treated areas is clearly visible in the SECM feedback mode image (Fig. 
7.9a). A lower current was measured over the bleached area probably due to the reduced AO activity. Some of the present AOs, such as anthocyanidin, are also pigments that change color or turn even colorless after oxidation (Fig. 7.9b). However, many AOs, such as GSH, are colorless and their degradation cannot be identified simply by eye or other optical methods, but by using the presented SECM imaging strategy.
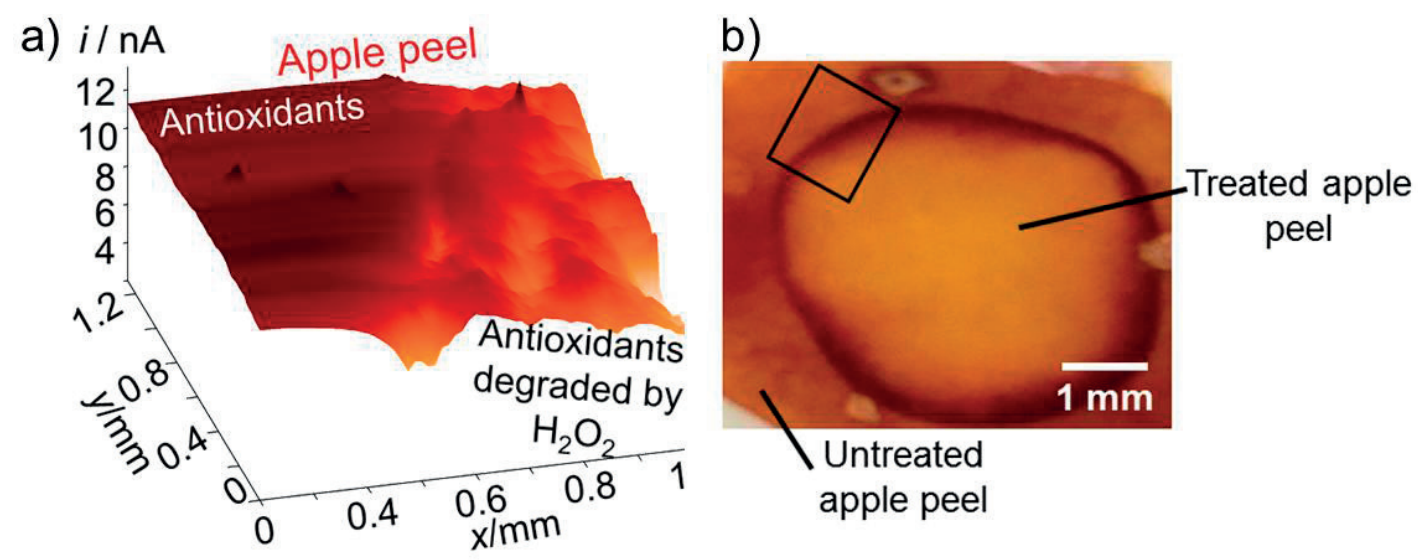

Fig. 7.9 a) Soft-Probe-SECM image of an apple peel area partially exposed to $\mathrm{H}_{2} \mathrm{O}_{2}$ for $15 \mathrm{~min}$ prior to the experiment. Experimental details: working potential $E_{\mathrm{T}}=0.4 \mathrm{~V}$, probe translation speed $=50 \mu \mathrm{m} / \mathrm{s}$, step size $=25 \mu \mathrm{m}, h_{\mathrm{P}}=-200 \mu \mathrm{m}$ and delay time between probe movement and current reading $=0.1 \mathrm{~s}, 2 \mathrm{mM} \mathrm{FcMeOH}$ in $10 \mathrm{mM}$ phosphate buffer (PB), $\mathrm{pH} 6 . \mathrm{b}$ ) Photograph of the investigated apple peel after treatment with $\mathrm{H}_{2} \mathrm{O}_{2}(60 \%)$. The area scanned by Soft-Probe-SECM is indicated by the black rectangle.

Furthermore, the higher content of AOs in the apple peel was confirmed by SECM feedback mode imaging of the cross-section of an apple (Fig. 7.10). After the cutting procedure, the sample was quickly embedded in a flat wax matrix and immersed in solution. Over the wax area, a pure hindered diffusion current was measured. Over the apple peel, the current gradient from higher values (lower part of the SECM image) to lower values (upper part of the SECM image) was due to slow and slight electrode fouling, most likely caused by compounds diffusing slowly out of the apple and contaminating the carbon electrode surface. The apple peel of approximately $100 \mu \mathrm{m}$ thickness (from right to left containing the cuticular, epidermal and hypodermal layers) shows a higher and homogeneous AO activity in respect to the flesh (Fig. 7.10a), which is also valid when considering the decaying electrode current and comparing the currents for each $y$-position. On the contrary, a locally heterogeneous gradient of AO activity from the peel towards the flesh can be seen. The probe slide well on wax and 
apple (step 1 and 4). However, when passing the gap, the probe would flip due to the pressure applied on the sample surface (step2-3). This would cause a peak in SECM image as schematically shown in Fig. 7.11. When the probe passed through the gap between apple and the wax, the vibration of the probe may cause edge effect and thus the current increased at the short moment. The photograph of the apple cross section after the SECM experiment is shown in Fig. 7.10b. Please note, that the gap between apple peel and wax in the optical image is larger than it was during the actual experiment due to the water loss during drying in air. This experiment demonstrates that Soft-Probe-SECM imaging can be used to investigate the distribution of the AO activity in various parts of the apple, even in the fruit part. Imaging on the flesh is more challenging since various biological compounds can diffuse in the electrolyte solution causing serious electrode contamination issues over time (seen as a minor current decrease in positive $y$-direction).

In order to show that the SECM image of the apple cross-section represents the global AO activity a freshly prepared apple cross-section was micro-contact printed onto a PVDF membrane to adsorb the hydrophobic AOs molecules and proteins (Fig. 7.10c). PVDF membranes are well known for their extraordinary ability to adsorb proteins and hydrophobic molecules (see also experimental part of this thesis). The left image part (i.e. $x=0-1.2 \mathrm{~mm}$ ) corresponds to the apple fruit, whereas the area between $x=1.2-1.3 \mathrm{~mm}$ shows the enhanced AO activity of the apple peel. This result is in very good agreement with the SECM image performed directly on the apple cross-section. However, the permeability for the redox mediator is expected to be higher when the exposed apple peel and flesh are immersed in solution compared to the experiments on the top of the peel. Because the AO activity of the peel and flesh was preserved in the printed sample, it can be suggested that the permeability of the redox mediator over the entire sample is comparable and/or does not play a role for this measurement. 


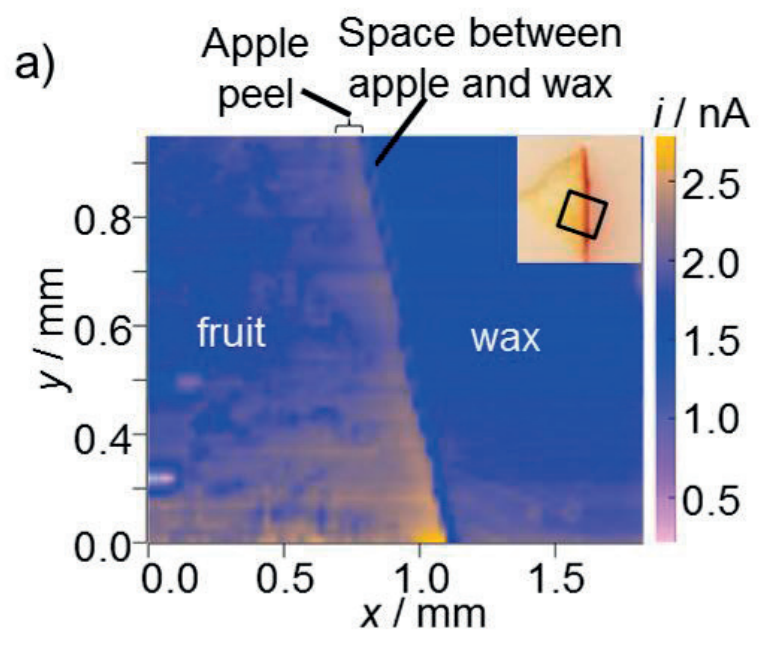

b)

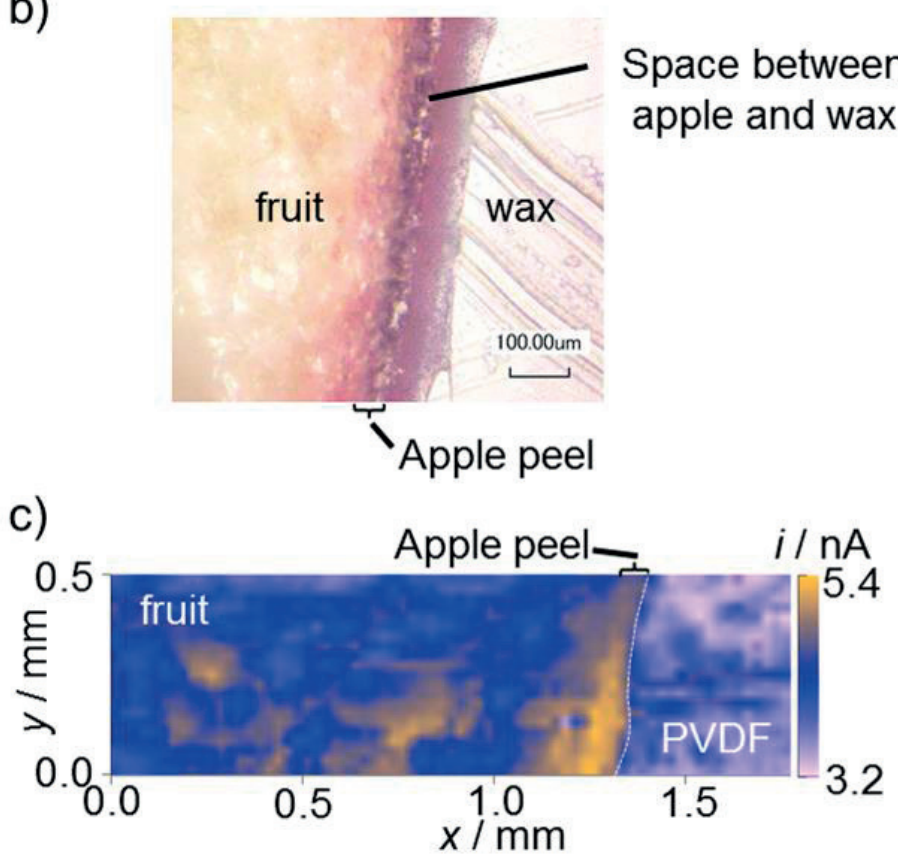

Space between apple and wax

Fig. 7.10 a) Soft-Probe-SECM feedback mode image of an apple cross-section. Photograph of the sample is in the right corner. The scanned area is indicated by the black rectangle. b) Microscopic picture of the apple cross-section. The thickness of the apple peel was around 50$70 \mu \mathrm{m}$. The image was recorded directly after the SECM experiment. The apple cross-section was already dried and contracted slightly compared to the situation during the SECM imaging procedure. c) Soft-Probe-SECM feedback image of an apple cross section micro-printed on a PVDF membrane. Experimental details: working potential $E_{\mathrm{T}}=0.4 \mathrm{~V}$, probe translation speed $=50 \mu \mathrm{m} / \mathrm{s}$, step size $=25 \mu \mathrm{m}, h_{\mathrm{P}}=-200 \mu \mathrm{m}$ and delay time between probe movement and current reading $=0.1 \mathrm{~s}, 2 \mathrm{mM} \mathrm{FcMeOH}$ in $10 \mathrm{mM} \mathrm{PB}, \mathrm{pH} 6$. 

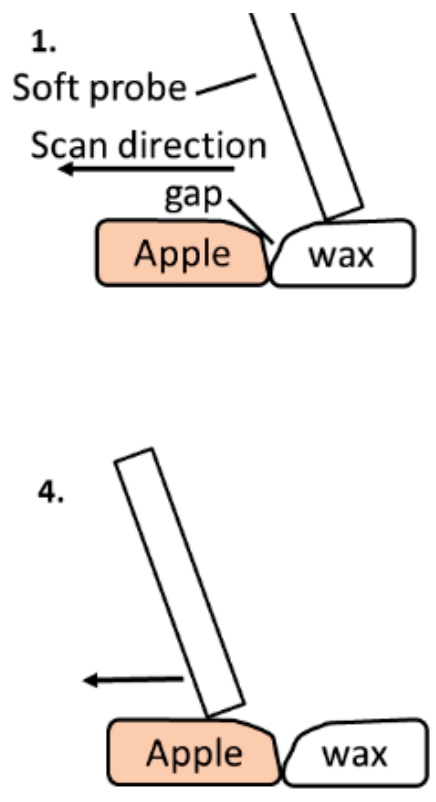

2.
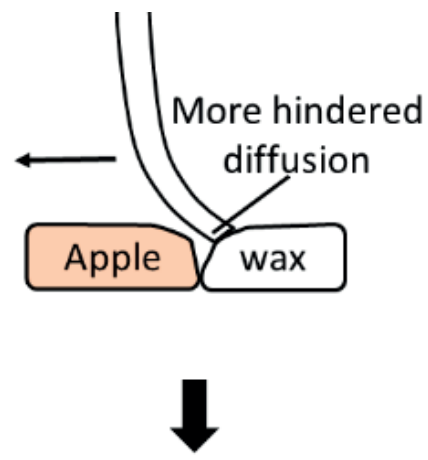

3.

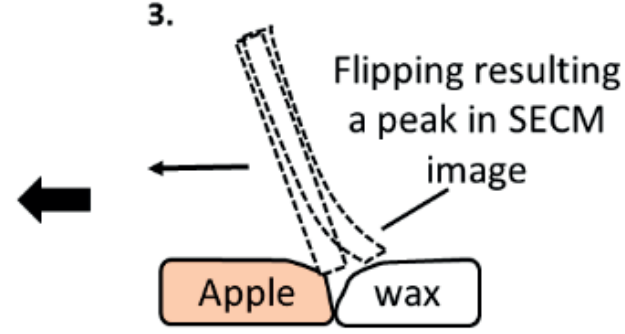

Fig. 7.11 Schematic representation of a soft probe passing the gap between apple and wax.

\subsection{Conclusions}

The local distribution of antioxidant activity on apple peels and within apple peel cross-sections was efficiently mapped using Soft-Probe-SECM. The SECM feedback mode was successfully applied to record an enhanced current over AO-rich locations due to the redox mediator regeneration of $\mathrm{FcMeOH}$ initiated by the $\mathrm{AO}$ defense system of the apple peel and flesh. To conclude, the AO activity on red apple peel cuticle (high), on the yellow lenticel areas (lower) and on areas with artificially degraded AO activity (very low) has been visualized. Furthermore, the higher and homogeneous AO activity in the peel compared to the flesh was confirmed. However, the possible influence of the peel permeability for the redox mediator needs to be considered for the interpretation of the presented data.

The presented electrochemical approach could be the basis for further research and development of electrochemically based in-field antioxidant sensors and scanners. Non-invasive monitoring on apple peels of intact apples could be achieved by implementing the soft microelectrode into portable, handhold scanners for in-field measurements. The electrolyte solutions could be inserted into a hydrogel or delivered and aspirated from microfluidic channels. Such scanners would be attractive for the 
monitoring of the antioxidant defense of apple peels during growing, picking and storing, for the early identification of fruit diseases, such as lenticel breakdown. 


\section{General Conclusions and Future Perspectives}

In this thesis, various applications of Soft-Probe-SECM for bio-imaging and biosensing of human, animal and plant tissue were demonstrated. Soft probes are easy to prepare and the SECM instrumentation can be kept simple and affordable bringing many advantages for tissue analysis. In Chapters 1 and 2, the fabrication of different types of soft probes including conventional single soft stylus, stair case shaped single soft probe, conventional soft linear array, and newly developed soft spider probe that contains eight microelectrodes independently can slide over rough surface features. Thanks to advanced laser microfabrication tools, the tips of the soft probes could be made significantly smaller allowing a more independent brushing of rough surfaces, such as thick tissue.

In Chapter 3, multiple methodologies for the detection of the melanoma biomarker tyrosinase were established based on conventional SECM instrumentation, i.e. a Pt microelectrode operated in constant height mode. In the first approach, the so-called "oxygen reduction method" enabled the indirect SECM detection of adsorbed proteins on PVDF membrane by measuring the different concentrations of dissolved oxygen about protein blocked empty hydrophobic PVDF pores. Secondly, SECM was implemented as a useful tool for the monitoring of the tyrosinase enzymatic activity by detecting locally the products of the enzymatic reaction between L-DOPA and tyrosinase. Finally, the specific recognition of tyrosinase was achieved via the SECM readout of an adapted immunoassay strategy for tyrosinase in the SG/TC mode. This concept could successfully be applied for the localization and activity mapping of tyrosinase inside banana peels where tyrosinase is present as well.

In Chapter 4, the knowledge towards tyrosinase mapping using SECM detection was applied to monitor the tyrosinase over-expression in skin biopsies from different melanoma stages. An immunoassay was employed to investigate tyrosinase inside nonmetastatic melanoma (stage II) and metastatic stage (stage III) using Soft-Probe-SECM. The imaging of different tyrosinase distribution patterns allows the distinguishing of these two cancer stages and normal skin. This work demonstrated the first example of SECM on thin human tissue biopsies as an alternative for melanoma diagnostics. Soft- 
Probe-SECM revealed the heterogeneity and reduced tyrosinase concentration with micrometer resolution, which macroscopic electrochemical techniques cannot provide. Compared to state-of-the-art microscopic techniques, Soft-Probe-SECM can avoid optical interferences caused by species such as melanin. However, although SECM instrumentation can be rather simple and affordable, its throughput needs to be sped up to compete with standard optical diagnostics.

In Chapters 5 and 6, Soft-Probe-SECM of thick tissues was addressed. Compared with the thin tissue slices that can be observed under optical microscopes, the thick tissues with rugged surface are usually difficult to deal with due to limited light penetration and varying focal points. The so-called "spider probe" comprised of eight independent soft microelectrodes brush easily in constant distance mode over such tissues and overcome problems in optical as well as conventional electrochemical probe microscopy. The biodistribution of naturally present biomarkers for melanoma (SECM SG/TC mode based on immunoassays), artificially induced nanomaterials (graphene oxide nanoribbons visualized by SECM FB mode), and redox active proteins were imaged in thick as well as large tissue, such as an entire mouse heart section.

In Chapter 7, the global activity of AOs, which are mainly polyphenols in apple peel, was analyzed using Soft-Probe-SECM. The different AO activities in the lenticel containing regions and intact, here red, apple skin was imaged. When a soft probe is brushed within a thin layer of liquid on a fresh apple, the technique can be made noninvasive and can open a new possibility of analyzing the AO defense of apples, which can be related to fruit diseases, storage or growth conditions.

The applications of Soft-Probe-SECM presented in this thesis pave not only the way to analyze a broad range of tissue sections, but also live material, including tissue and also live cells (Fig. 8.1). For single cell analysis, Bio-SECM can be coupled with optical microscopy. This possesses the advantages of real time observation, combining fluorescence or visible light measurements with electrochemical scanning. ${ }^{[282]}$ Although soft probes have demonstrated their ability to scan delicate samples such as human tissue in contact mode, the scanning of single live cells is still challenging and requires even softer probes as developed herein. Single live cells are simple to detach, extremely vulnerable and sensitive to pressure, since they are not embedded in a relatively stable tissue matrix. The physical impact on the cell needs to be minimized and further work needs to be done to optimize the probe fabrication for this purpose. 


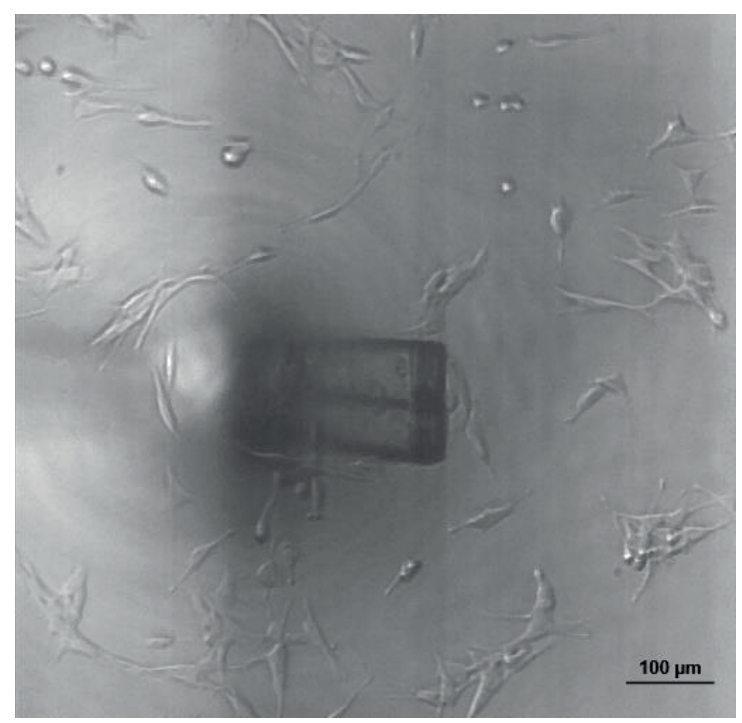

Fig. 8.1 A microscopic image of the soft electrode implanted in Bio-SECM scanning over live cells.

In recent years, the miniaturization of biosensing device enables the minimal invasive clinical detection of various biomolecules such as biomarkers, ions, and glucose. Microneedles related devices draw scientists' attention because they can be employed on transdermal biosensing of analytes of clinical interest. ${ }^{[283-287]}$ Microneedles are miniaturized replica of hypodermic needles with length-scales of hundreds of micrometers with the advantages of pain-free, easy-to-use and minimaltraining features. For the future, it would be of value to integrate electrochemical detection with a microneedle system into a small real-time sensor. The electrodes could be located at the tip of the microneedle as shown in Fig. 8.2. Antibodies or aptamers will be immobilized on the WE surface within a gel or porous matrix to provide selectivity for the detection of certain markers, such as tyrosinase and s100 protein. The aim of this device will be to diagnose skin cancer through addressable microneedles and thus locate the cancer area on the skin. Further treatment can be achieved by using electroporation applying on the cancer area or by other local treatment methods. For instance, the microfluidic system can be integrated in the microneedle system, enabling the local release of the drug. If this device is developed successfully, it can be used in remote area where lack of hospitals, doctors and medical sources such as mountain areas or space station in outer space. 


\section{Microneedle array}

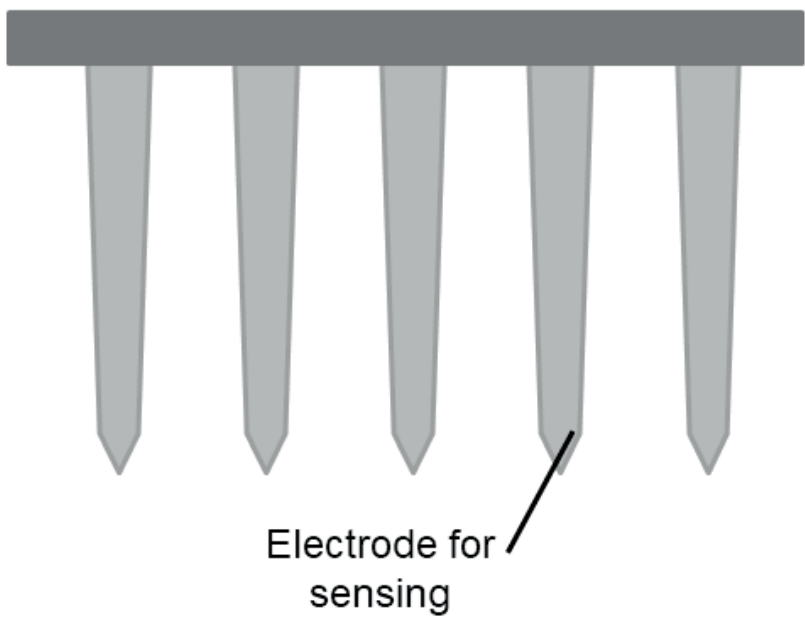

Fig. 8.2 Schematic representation of the microneedles sensing probe with electrode integrated at the tip. 


\section{References}

[1] A. J.Bard, F. R. F.Fan, M.V.Mirkin, Electroanal. Chem. 1994, 18, 357-363.

[2] P. R.Unwin, A. J.Bard, J. Phys. Chem 1991, 95, 7814-7824.

[3] K.Hu, Y.Gao, Y.Wang, Y.Yu, X.Zhao, S. A.Rotenberg, E.Gökmese, M.V.Mirkin, G.Friedman, Y.Gogotsi, J. Solid State Electrochem. 2013, 17, 2971-2977.

[4] C.Kranz, Analyst 2014, 139, 336-52.

[5] M.Etienne, E. C.Anderson, S. R.Evans, W.Schuhmann, I.Fritsch, Anal. Chem. 2006, 78, 7317-7324.

[6] R. C.Engstrom, M.Weber, D. J.Wunder, R.Burgess, S.Winquist, Anal. Chem. 1986, 58, 844-848.

[7] H.Liu, F.Fan, C.Lin, A.Bard, J. Am. Chem. Soc. 1986, 108, 3838-3839.

[8] J.Kwak, A. J.Bard, Anal. Chem. 1989, 61, 1221-1227.

[9] A.Bondarenko, T.-E.Lin, P.Stupar, A.Lesch, F.Cortés-Salazar, H. H.Girault, H.Pick, Anal. Chem. 2016, 88, 11436-11443.

[10] T.-E.Lin, A.Lesch, C.-L.Li, H. H.Girault, J. Electroanal. Chem. 2017, 786, $120-128$.

[11] T.-E.Lin, F.Cortés-Salazar, A.Lesch, L.Qiao, A.Bondarenko, H. H.Girault, Electrochim. Acta 2015, 179, 57-64.

[12] T.-E.Lin, A.Bondarenko, A.Lesch, H.Pick, F.Cortés-Salazar, H. H.Girault, Angew. Chemie - Int. Ed. 2016, 55, 3813-3816.

[13] H.Kikuchi, A.Prasad, R.Matsuoka, S.Aoyagi, T.Matsue, S.Kasai, Front. Physiol. 2016, 7, 25.

[14] A.Page, M.Kang, A.Armitstead, D.Perry, P. R.Unwin, Anal. Chem. 2017, 89, acs.analchem.6b04629.

[15] S.Bergner, P.Vatsyayan, F.Matysik, Anal. Chim. Acta 2013, 775, 1-13.

[16] J.V.Perales-Rondón, E.Herrero, J.Solla-Gullón, C. M.Sánchez-Sánchez, V.Vivier, J. Electroanal. Chem. 2017, 793, 218-225.

[17] A.Sridhar, A.van denBerg, S.LeGac, Electroanalysis 2014, 26, 1881-1885.

[18] A.Sridhar, H. L.deBoer, A.van denBerg, S.LeGac, PLoS One 2014, 9, e93618.

[19] T.Arai, T.Nishijo, Y.Matsumae, Y.Zhou, K.Ino, H.Shiku, T.Matsue, Anal. Chem. 2013, 85, 9647-9654.

[20] F.Li, P. R.Unwin, J. Phys. Chem. C 2015, 119, 4031-4043.

[21] C.Demaille, M.Brust, M.Tsionsky, A.J.Bard, Anal. Chem. 1997, 69, 2323-8.

[22] C.Amatore, C.Pebay, L.Thouin, A.Wang, J. S.Warkocz, Anal. Chem. 2010, 82, 6933-6939.

[23] M.V.Mirkin, B. R.Horrocks, Anal. Chim. Acta 2000, 406, 119-146. 
[24] J.Heinze, Angew. Chemie Int. Ed. English 1993, 32, 1268-1288.

[25] C. G.Zoski, Electroanalysis 2002, 14, 1041-1051.

[26] J.Heinze, Angew. Chemie Int. Ed. English 1993, 32, 1268-1288.

[27] D.Shoup, A.Szabo, J. Electroanal. Chem. 1982, 140, 237-245.

[28] R. B.Keithley, P.Takmakov, E. S.Bucher, A. M.Belle, C. A.Owesson-White, J.Park, R. M.Wightman, Anal. Chem. 2011, 83, 3563-3571.

[29] B. E. K.Swamy, B. J.Venton, Anal. Chem. 2007, 79, 744-750.

[30] H.Yang, A. J.Bard, J. Electroanal. Chem. 1992, 339, 423-449.

[31] M.Etienne, Y.Guillemin, D.Grosso, A.Walcarius, Anal. Bioanal. Chem. 2013, 405, 1497-1512.

[32] J. H.Zhou, J. P.Zhao, L. K.Wu, J. M.Hu, Electrochem. commun. 2016, 72, 171175.

[33] J.Molina, J.Fernández, F.Cases, Synth. Met. 2016, 222, 145-161.

[34] L.Stratmann, J.Clausmeyer, W.Schuhmann, ChemPhysChem 2015, 16, 34773482 .

[35] K.Borgwarth, C.Ricken, D. G.Ebling, J.Heinze, Berichte der Bunsengesellschaft für Phys. Chemie 1995, 99, 1421-1426.

[36] S.-Y.Ku, K.-T.Wong, A. J.Bard, J. Am. Chem. Soc. 2008, 130, 2392-2393.

[37] A. J.Bard, M.V.Mirkin, Scanning Electrochemical Microscopy, Second Edition, CRC Press, 2012.

[38] K.Torbensen, M.Kongsfelt, K.Shimizu, E. B.Pedersen, T.Skrydstrup, S. U.Pedersen, K.Daasbjerg, Langmuir 2015, 31, 4443-4452.

[39] T. M.Day, P. R.Unwin, J.V.Macpherson, Nano Lett. 2007, 7, 51-57.

[40] W.Liu, F.Cao, Y.Xia, L.Chang, J.Zhang, Electrochim. Acta 2014, 132, 377388.

[41] A.Lesch, B.Vaske, F.Meiners, D.Momotenko, F.Cortés-Salazar, H. H.Girault, G.Wittstock, Angew. Chemie - Int. Ed. 2012, 51, 10413-10416.

[42] C.Combellas, M.Fermigier, A.Fuchs, F.Kanoufi, Anal. Chem. 2005, 77, 79667975.

[43] G.Wittstock, M.Burchardt, S. E.Pust, Y.Shen, C.Zhao, Angew. Chemie - Int. Ed. 2007, 46, 1584-1617.

[44] P.Sun, F. O.Laforge, M.V.Mirkin, Phys. Chem. Chem. Phys. 2007, 9, 802-823.

[45] M.V.Mirkin, W.Nogala, J.Velmurugan, Y.Wang, Phys. Chem. Chem. Phys. 2011, 13, 21196.

[46] A. J.Bard, M.VMirkin, P. R.Unwin, D. O.Wipf, J. Phys. Chem. 1992, 96, 1861-1868.

[47] R.Cornut, C.Lefrou, J. Electroanal. Chem. 2008, 621, 178-184.

[48] S. S.Jamali, S. E.Moulton, D. E.Tallman, M.Forsyth, J.Weber, G. G.Wallace, 
Electrochim. Acta 2015, 152, 294-301.

[49] S. S.Jamali, S. E.Moulton, D. E.Tallman, M.Forsyth, J.Weber, G. G.Wallace, Corros. Sci. 2014, 86, 93-100.

[50] D.Polcari, P.Dauphin-Ducharme, J.Mauzeroll, Chem. Rev. 2016, 116, 1323413278.

[51] B. R.Horrocks, D.Schmidtke, A.Heller, A. J.Bard, Anal. Chem. 1993, 65, 3605-3614.

[52] S.Amemiya, J.Guo, H.Xiong, D. A.Gross, Anal. Bioanal. Chem. 2006, 386, 458-471.

[53] Y.Matsumae, Y.Takahashi, K.Ino, H.Shiku, T.Matsue, Anal. Chim. Acta 2014, $842,20-26$.

[54] Y.Takahashi, A. I.Shevchuk, P.Novak, B.Babakinejad, J.Macpherson, P. R.Unwin, H.Shiku, J.Gorelik, D.Klenerman, Y. E.Korchev, et al., Proc. Natl. Acad. Sci. U. S. A. 2012, 109, 11540-5.

[55] H.Yamada, D.Haraguchi, K.Yasunaga, Anal. Chem. 2014, 86, 8547-8552.

[56] S. E.Salamifar, R. Y.Lai, Anal. Chem. 2013, 85, 9417-9421.

[57] V. S.Joshi, J.Kreth, D.Koley, Anal. Chem. 2017, 89, acs.analchem.7b01677.

[58] Y.Takahashi, T.Miyamoto, H.Shiku, R.Asano, T.Yasukawa, I.Kumagai, T.Matsue, Anal. Chem. 2009, 81, 2785-2790.

[59] S. A.Rotenberg, M.V.Mirkin, J. Mammary Gland Biol. Neoplasia 2004, 9 , 375-382.

[60] A. B.Chinen, C. M.Guan, J. R.Ferrer, S. N.Barnaby, T. J.Merkel, C. A.Mirkin, Chem. Rev. 2015, 115, 10530-10574.

[61] S.Zhang, J.Yang, J.Lin, Bioelectrochemistry 2008, 72, 47-52.

[62] R. M.Wightman, Science 2006, 311, 1570-1574.

[63] A.Schulte, W.Schuhmann, Angew. Chemie - Int. Ed. 2007, 46, 8760-8777.

[64] P.Knittel, H.Zhang, C.Kranz, G. G.Wallace, M. J.Higgins, Nanoscale 2016, 8, 4475-4481.

[65] C. a.Morris, C.-C.Chen, L. a.Baker, Analyst 2012, 137, 2933-2938.

[66] J. J.Santana, M.Pähler, R. M.Souto, W.Schuhmann, Electrochim. Acta 2012 , 77, 60-64.

[67] J.Izquierdo, B. M.Fernández-Pérez, A.Eifert, R. M.Souto, C.Kranz, Electrochim. Acta 2015, 201, 320-332.

[68] P. M.Diakowski, Z.Ding, Phys. Chem. Chem. Phys. 2007, 9, 5966-74.

[69] U. M.Tefashe, G.Wittstock, Comptes Rendus Chim. 2013, 16, 7-14.

[70] F.Cortés-Salazar, D.Momotenko, A.Lesch, G.Wittstock, H. H.Girault, Anal. Chem. 2010, 82, 10037-10044.

[71] A.Lesch, D.Momotenko, F.Cortés-Salazar, F.Roelfs, H. H.Girault, 
G.Wittstock, Electrochim. Acta 2013, 110, 30-41.

[72] F.Cortés-Salazar, D.Momotenko, A.Lesch, G.Wittstock, H. H.Girault, Anal. Chem. 2010, 82, 10037-10044.

[73] D.Momotenko, F.Cortes-Salazar, A.Lesch, G.Wittstock, H. H.Girault, Anal. Chem. 2011, 83, 5275-5282.

[74] F.Cortes-Salazar, A.Lesch, D.Momotenko, J.-M.Busnel, G.Wittstock, H. H.Girault, Anal. Methods 2010, 2, 817-823.

[75] J.-S.Taylor, Science 2015, 347, 31-35.

[76] A. J.Miller, M. C.Mihm, N. Engl. J. Med. 2006, 355, 51-65.

[77] I.Koskivuo, Forum Nord. Dermato-Venerology 2010, 15, 47-48.

[78] C. M.Balch, J. E.Gershenwald, S. J.Soong, J. F.Thompson, M. B.Atkins, D. R.Byrd, A. C.Buzaid, A. J.Cochran, D. G.Coit, S.Ding, et al., J. Clin. Oncol. 2009, 27, 6199-6206.

[79] B. E. G.Rothberg, M. B.Bracken, D. L.Rimm, J. Natl. Cancer Inst. 2009, 101, 452-474.

[80] V.Gray-Schopfer, C.Wellbrock, R.Marais, Nature 2007, 445, 851-857.

[81] A. B.Lerner, Arch. Biochem. Biophys. 1952, 36, 473-481.

[82] S. Y.Seo, V. K.Sharma, N.Sharma, J. Agric. Food Chem. 2003, 51, 2837-2853.

[83] V. J.Hearing, K.Tsukamoto, FASEB J. 1991, 5, 2902-2909.

[84] V. J.Hearing, M.Jiménez, Int. J. Biochem. 1987, 19, 1141-1147.

[85] M.Seiberg, Pigment cell Res. 2001, 14, 236-42.

[86] M.Brenner, V. J.Hearing, Photochem. Photobiol. 2008, 84, 539-549.

[87] J.-P.Ortonne, Br. J. Dermatol. 2002, 146, 7-10.

[88] A.Kastner, E. C.Hirsch, O.Lejeune, F.Javoy-Agid, O.Rascol, Y.Agid, J. Neurochem. 1992, 59, 1080-1089.

[89] G. E.Orchard, Histochem. J. 2000, 32, 475-481.

[90] G. F. L.Hofbauer, J.Kamarashev, R.Geertsen, R.Böni, R.Dummer, J. Cutan. Pathol. 1998, 25, 204-209.

[91] N.Abbasi, H.Shaw, D.Rigel, JAMA J. Med. Assoc. 2004, 292, 2771-2776.

[92] G. C.Bethune, A. S. L.Pettit, D.Veldhuijzen van Zanten, P. J.Barnes, Histopathology 2017, 70, 966-974.

[93] H.Frickmann, A. E.Zautner, A.Moter, J.Kikhney, R. M.Hagen, H.Stender, S.Poppert, Crit. Rev. Microbiol. 2017, 7828, 1-31.

[94] N.Vishwanathan, A.Bandyopadhyay, H. Y.Fu, K. C.Johnson, N. M.Springer, W. S.Hu, Biotechnol. Bioeng. 2017, 114, 1903-1908.

[95] A. A.Marghoob, L. D.Swindle, C. Z. M.Moricz, F. A.Sanchez Negron, B.Slue, A. C.Halpern, A. W.Kopf, J. Am. Acad. Dermatol. 2003, 49, 777-797.

[96] A.Gadeliya Goodson, D.Grossman, J. Am. Acad. Dermatol. 2009, 60, 719-735. 
[97] T.Wadhawan, N.Situ, K.Lancaster, X.Yuan, G.Zouridakis, in Proc. - Int. Symp. Biomed. Imaging, IEEE, 2011, pp. 133-136.

[98] G.Zonios, A.Dimou, I.Bassukas, D.Galaris, A.Tsolakidis, E.Kaxiras, J. Biomed. Opt. 2008, 13, 14017.

[99] X.Wang, H.Li, X.Li, Y.Chen, Y.Yin, G.Li, Electrochem. commun. 2014, 39, $12-14$.

[100] R.Baron, M.Zayats, I.Willner, Anal. Chem. 2005, 77, 1566-1571.

[101] X.Ren, T.Yan, Y.Zhang, D.Wu, H.Ma, H.Li, B.Du, Q.Wei, Biosens. Bioelectron. 2014, 58, 345-350.

[102] R.Seenivasan, N.Maddodi, V.Setaluri, S.Gunasekaran, Biosens. Bioelectron. 2015, 68, 508-515.

[103] S. B.Revin, S. A.John, Sensors Actuators, B Chem. 2013, 188, 1026-1032.

[104] S. A.Sydlik, S.Jhunjhunwala, M. J.Webber, D. G.Anderson, R.Langer, ACS Nano 2015, 9, 3866-3874.

[105] B. C.Thompson, E.Murray, G. G.Wallace, Adv. Mater. 2015, 27, 7563-7582.

[106] K. A.Mkhoyan, A. W.Contryman, J.Silcox, D. A.Stewart, G.Eda, C.Mattevi, S.Miller, M.Chhowalla, Nano Lett. 2009, 9, 1058-1063.

[107] M.Xu, T.Liang, M.Shi, H.Chen, Chem. Rev. 2013, 113, 3766-3798.

[108] J.Lin, X.Chen, P.Huang, Adv. Drug Deliv. Rev. 2016, 105, 242-254.

[109] D. R.Dreyer, S.Park, C. W.Bielawski, R. S.Ruoff, Chem. Soc. Rev. 2010, 39, $228-240$.

[110] K.Yang, L.Feng, Z.Liu, Adv. Drug Deliv. Rev. 2016, 105, 228-241.

[111] Z.Liu, J. T.Robinson, X.Sun, H.Dai, J. Am. Chem. Soc. 2008, 130, 1087610877.

[112] W.Zhang, Z.Guo, D.Huang, Z.Liu, X.Guo, H.Zhong, Biomaterials 2011, 32, $8555-8561$.

[113] J.Joo, E. J.Kwon, J.Kang, M.Skalak, E. J.Anglin, A. P.Mann, E.Ruoslahti, S.Bhatia, M. J.Sailor, Nanoscale Horiz. 2016, 1, 1-8.

[114] D.Yang, L.Feng, C. A.Dougherty, K. E.Luker, D.Chen, M. A.Cauble, M. M.Banaszak Holl, G. D.Luker, B. D.Ross, Z.Liu, et al., Biomaterials 2016, 104, 361-371.

[115] S.Kim, S.-R.Ryoo, H.-K.Na, Y.-K.Kim, B.-S.Choi, Y.Lee, D.-E.Kim, D.H.Min, Chem. Commun. 2013, 49, 8241-3.

[116] R.-C.Huang, W.-J.Chiu, I.Po-Jung Lai, C.-C.Huang, Sci. Rep. 2015, 5, 10292.

[117] Q.Liu, L.Wei, J.Wang, F.Peng, D.Luo, R.Cui, Y.Niu, X.Qin, Y.Liu, H.Sun, et al., Nanoscale 2012, 4, 7084-9.

[118] Q.Liu, B.Guo, Z.Rao, B.Zhang, J. R.Gong, Nano Lett. 2013, 13, 2436-2441.

[119] X.Hu, X.Dong, Y.Lu, J.Qi, W.Zhao, W.Wu, Drug Discov. Today 2017, 22, 
$382-387$.

[120] C.Tan, J.Rodríguez-López, J. J.Parks, N. L.Ritzert, D. C.Ralph, H. D.Abruña, ACS Nano 2012, 6, 3070-3079.

[121] S.Rapino, E.Treossi, V.Palermo, M.Marcaccio, F.Paolucci, F.Zerbetto, Chem. Commun. 2014, 50, 13117-13120.

[122] C. L.Sun, C. T.Chang, H. H.Lee, J.Zhou, J.Wang, T. K.Sham, W. F.Pong, ACS Nano 2011, 5, 7788-7795.

[123] C.-W.Lin, K.-C.Wei, S.Liao, C.-Y.Huang, C.-L.Sun, P.-J.Wu, Y.-J.Lu, H.W.Yang, C.-C. M.Ma, Biosens. Bioelectron. 2015, 67, 431-437.

[124] P.Verboven, A.Nemeth, M. K.Abera, E.Bongaers, D.Daelemans, P.Estrade, E.Herremans, M.Hertog, W.Saeys, E.Vanstreels, et al., Postharvest Biol. Technol. 2013, 78, 123-132.

[125] D.Post-Beittenmiller, Plant Biol. 1996, 47, 405-430.

[126] Z.Ju, W. J.Bramlage, Postharvest Biol. Technol. 1999, 16, 107-118.

[127] A.Lesch, F.Cortés-Salazar, M.Prudent, J.Delobel, S.Rastgar, N.Lion, J. D.Tissot, P.Tacchini, H. H.Girault, J. Electroanal. Chem. 2014, 717-718, 6168.

[128] C. G.Zoski, Electroanalysis 2002, 14, 1041-1051.

[129] G.Wittstock, T.Asmus, T.Wilhelm, Fresenius. J. Anal. Chem. 2000, 367, 34651.

[130] M.Zhang, A.Becue, M.Prudent, C.Champod, H. H.Girault, Chem. Commun. 2007, 0, 3948.

[131] Y.Jiang, X.Duan, D.Joyce, Z.Zhang, J.Li, Food Chem. 2004, 88, 443-446.

[132] V.Kahn, J. Food Sci. 2006, 50, 111-115.

[133] Y.-H.Song, E.Connor, Y.Li, B.Zorovich, P.Balducci, N.Maclaren, Lancet 1994, 344, 1049-1052.

[134] Y.-T.Chen, E.Stockert, S.Tsang, K. A.Coplan, L. J.Old, Immunology 1995, 92, $8125-8129$.

[135] M.Mossberg, S.Vernick, R.Ortenberg, G.Markel, Y.Shacham-Diamand, J.Rishpon, Electroanalysis 2014, 26, 1671-1675.

[136] M.Urosevic, B.Braun, J.Willers, G.Burg, R.Dummer, Exp. Dermatol. 2005, 14, 491-497.

[137] G. E.Orchard, Histochem. J. 2000, 32, 475-481.

[138] X.Feng, F.Feng, M.Yu, F.He, Q.Xu, H.Tang, S.Wang, Y.Li, D.Zhu, Org. Lett. 2008, 10, 5369-5372.

[139] Q.Xu, J.Yoon, Chem. Commun. 2011, 47, 12497-9.

[140] C.Védrine, S.Fabiano, C.Tran-Minh, Talanta 2003, 59, 535-544.

[141] P.Önnerfjord, J.Emnéus, G.Marko-Varga, L.Gorton, F.Ortega, E.Domínguez, 
Biosens. Bioelectron. 1995, 10, 607-619.

[142] B.Serra, M.Dolores Morales, J.Zhang, A. J.Reviejo, E. H.Hall, J. M.Pingarron, Anal. Chem. 2005, 77, 8115-8121.

[143] S.Cosnier, S.Szunerits, R. S.Marks, J. P.Lellouche, K.Perie, J. Biochem. Biophys. Methods 2001, 50, 65-77.

[144] H. J.Issaq, Electrophoresis 2001, 22, 3629-3638.

[145] K.Berggren, T. H.Steinberg, W. M.Lauber, J. A.Carroll, M. F.Lopez, E.Chernokalskaya, L.Zieske, Z.Diwu, R. P.Haugland, W. F.Patton, Anal. Biochem. 1999, 276, 129-143.

[146] T.Rabilloud, L.Vuillard, C.Gilly, J. J.Lawrence, 2009.

[147] V.Neuhoff, N.Arold, D.Taube, W.Ehrhardt, Electrophoresis 1988, 9, 255-262.

[148] M.Zhang, H. H.Girault, Analyst 2009, 134, 25-30.

[149] G.Wittstock, K.-J.Yu, H. B.Halsall, T. H.Ridgway, W. R.Heineman, Anal. Chem. 1995, 67, 3578-3582.

[150] A.Kueng, C.Kranz, A.Lugstein, E.Bertagnolli, B.Mizaikoff, Angew. Chemie Int. Ed. 2003, 42, 3238-3240.

[151] D. T.Pierce, P. R.Unwin, A. J.Bard, Anal. Chem 1992, 64, 1795-1804.

[152] S.Kasai, A.Yokota, H.Zhou, M.Nishizawa, K.Niwa, T.Onouchi, T.Matsue, Anal. Chem. 2000, 72, 5761-5765.

[153] G.Sciutto, S.Prati, R.Mazzeo, M.Zangheri, A.Roda, L.Bardini, G.Valenti, S.Rapino, M.Marcaccio, Anal. Chim. Acta 2014, 831, 31-37.

[154] D.Rudolph, D.Bates, T. J.DiChristina, B.Mizaikoff, C.Kranz, Electroanalysis 2016, 28, 2459-2465.

[155] H.Shiku, T.Matsue, I.Uchida, Anal. Chem. 1996, 68, 1276-1278.

[156] J.-M.Noel, A.Latus, C.Lagrost, E.Volanschi, P.Hapiot, J. Am. Chem. Soc. 2012, 134, 2835-2841.

[157] B. D.Bath, R. D.Lee, H. S.White, E. R.Scott, Anal. Chem. 1998, 70, 10471058.

[158] M.Gonsalves, A. L.Barker, J.V.Macpherson, P. R.Unwin, D.O’Hare, C. P.Winlove, Biophys. J. 2000, 78, 1578-1588.

[159] C. M.Sánchez-Sánchez, A. J.Bard, Anal. Chem. 2009, 81, 8094-8100.

[160] L.Ma, H.Zhou, S.Xin, C.Xiao, F.Li, S.Ding, Electrochim. Acta 2015, 178, 767777.

[161] M.Nebel, S.Grützke, N.Diab, A.Schulte, W.Schuhmann, Angew. Chemie - Int. Ed. 2013, 52, 6335-6338.

[162] K. B.Holt, A. J.Bard, Biochemistry 2005, 44, 13214-13223.

[163] T.Kaya, Y. S.Torisawa, D.Oyamatsu, M.Nishizawa, T.Matsue, Biosens. Bioelectron. 2003, 18, 1379-1383. 
[164] N.Géza, N.Lívia, in Trends Bioelectroanal., Springer, Cham, 2017, pp. 281339.

[165] T. C.Rohner, J. S.Rossier, H. H.Girault, Electrochem. commun. 2002, 4, 695700.

[166] F.Cortés-Salazar, J.-M.Busnel, F.Li, H. H.Girault, J. Electroanal. Chem. 2009, $635,69-74$.

[167] M.Zhang, G.Wittstock, Y.Shao, H. H.Girault, Anal. Chem. 2007, 79, 48334839.

[168] B. K.Sørensen, P.Højrup, E.Østergård, C. S.Jørgensen, J.Enghild, L. R.Ryder, G.Houen, Anal. Biochem. 2002, 304, 33-41.

[169] J.Ji, F.Liu, N. A.Hashim, M. R. M.Abed, K.Li, React. Funct. Polym. 2015, 86, $134-153$.

[170] A.Dobrzeniecka, A.Zeradjanin, J.Masa, J.Stroka, M.Goral, W.Schuhmann, P. J.Kulesza, in ECS Trans., The Electrochemical Society, 2011, pp. 33-44.

[171] S.Gidanian, P. J.Farmer, J. Inorg. Biochem. 2002, 89, 54-60.

[172] G.Volpe, R.Draisci, G.Palleschi, D.Compagnone, Analyst 1998, 123, 13031307.

[173] M.Jović, Y.Zhu, A.Lesch, A.Bondarenko, F.Cortés-Salazar, F.Gumy, H. H.Girault, J. Electroanal. Chem. 2017, 786, 69-76.

[174] B.Liu, S. A.Rotenberg, M.V.Mirkin, Proc. Natl. Acad. Sci. 2000, 97, 98559860.

[175] M.Nebel, S.Grützke, N.Diab, A.Schulte, W.Schuhmann, Angew. Chemie Int. Ed. 2013, 52, 6335-6338.

[176] S.Rapino, R.Marcu, A.Bigi, A.Soldà, M.Marcaccio, F.Paolucci, P. G.Pelicci, M.Giorgio, Electrochim. Acta 2015, 179, 65-73.

[177] R.Zhu, S. M.Macfie, Z.Ding, J. Exp. Bot. 2005, 56, 2831-2838.

[178] M.Tsionsky, Z. G.Cardon, A. J.Bard, R. B.Jackson, Plant Physiol 1997, 113, 895-901.

[179] H.Zhou, H.Shiku, S.Kasai, H.Noda, T.Matsue, H.Ohya-Nishiguchi, H.Kamada, Bioelectrochemistry 2001, 54, 151-156.

[180] E. R.Scott, H. S.White, J. B.Phipps, Anal. Chem. 1993, 65, 1537-1545.

[181] G. F. L.Hofbauer, J.Kamarashev, R.Geertsen, R.Böni, R.Dummer, J. Cutan. Pathol. 1998, 25, 204-209.

[182] F. H.Igney, P. H.Krammer, J. Leukoc. Biol. 2002, 71, 907-920.

[183] P.Alexander, Cancer Res. 1974, 34.

[184] J.Yan, C.Tingey, R.Lyde, T. C.Gorham, D. K.Choo, A.Muthumani, D.Myles, L. P.Weiner, K. A.Kraynyak, E. L.Reuschel, et al., Cancer Gene Ther. 2014, $21,507-517$. 
[185] M.Wirth, S.Villeneuve, C. M.Haase, C. M.Madison, H.Oh, S. M.Landau, G. D.Rabinovici, W. J.Jagust, JAMA Neurol. 2013, 70, 1512-9.

[186] C. R.Jack, D. M.Holtzman, Neuron 2013, 80, 1347-1358.

[187] D.Chernoff, R.Bolce, C.Hwang, X.Wang, A.Kivitz, J.Curtis, Ann. Rheum. Dis. 2017, 76, 232-233.

[188] B. E. G.Rothberg, M. B.Bracken, D. L.Rimm, JNCI J. Natl. Cancer Inst. 2009, $101,452-474$.

[189] A. K.Bosserhoff, Clin. Chim. Acta 2006, 367, 28-35.

[190] V. H.Stell, H. J.Norton, K. S.Smith, J. C.Salo, R. L.White, Ann. Surg. Oncol. 2007, 14, 893-898.

[191] J. C.Yang, R. M.Sherry, S. A.Rosenberg, Nat. Rev. Clin. Oncol. 2014, 11, 245246.

[192] R.Fernandez-Gonzalez, A.Jones, E.Garcia-Rodriguez, P. Y.Chen, A.Idica, S. J.Lockett, M. H.Barcellos-Hoff, C.Ortiz-De-Solorzano, Microsc. Res. Tech. 2002, 59, 522-530.

[193] P.Bajcsy, S. C.Lee, A.Lin, R.Folberg, J. Microsc. 2006, 221, 30-45.

[194] Z. S.Tannous, M. C.Mihm, T. J.Flotte, S.González, J. Am. Acad. Dermatol. 2002, 46, 260-263.

[195] S.Chatterjee, J. Oral Maxillofac. Pathol. 2014, 18, S111-6.

[196] W.Liu, H.Ye, A. J.Bard, J Phys Chem C 2009, 114, 1201-1207.

[197] M.Black, J.Cooper, P.McGinn, Meas. Sci. Technol. 2005, 16, 174-182.

[198] D.Momotenko, J. C.Byers, K.McKelvey, M.Kang, P. R.Unwin, ACS Nano 2015, 9, 8942-8952.

[199] M.Kang, D.Momotenko, A.Page, D.Perry, P. R.Unwin, Langmuir 2016, 32, 7993-8008.

[200] D.Momotenko, K.McKelvey, M.Kang, G. N.Meloni, P. R.Unwin, Anal. Chem. 2016, 88, 2838-2846.

[201] C. G.Zoski, N.Simjee, O.Guenat, M.Koudelka-Hep, Anal. Chem. 2004, 76, 6272.

[202] F.Cortés-Salazar, D.Momotenko, H. H.Girault, A.Lesch, G.Wittstock, Anal. Chem. 2011, 83, 1493-1499.

[203] R. J.Fasching, Y.Tao, F. B.Prinz, in Sensors Actuators, B Chem., 2005, pp. 964-972.

[204] A.Lesch, P. C.Chen, F.Roelfs, C.Dosche, D.Momotenko, F.Cortés-Salazar, H. H.Girault, G.Wittstock, Anal. Chem. 2014, 86, 713-720.

[205] A. L.Barker, P. R.Unwin, J. W.Gardner, H.Rieley, Electrochem. commun. 2004, 6, 91-97.

[206] L.Setti, A.Fraleoni-Morgera, I.Mencarelli, A.Filippini, B.Ballarin, M.DiBiase, 
Sensors Actuators, B Chem. 2007, 126, 252-257.

[207] N.Abbaspour, R.Hurrell, R.Kelishadi, J. Res. Med. Sci. 2014, 19, 164-174.

[208] J. F.Storz, J. Exp. Biol. 2016, 219, 3190-3203.

[209] C. J.Coates, H.Decker, Cell. Mol. Life Sci. 2016, 74, 293-317.

[210] F.Shi, W.Zheng, W.Wang, F.Hou, B.Lei, Z.Sun, W.Sun, Biosens. Bioelectron. 2014, 64, 131-137.

[211] Q.Wang, G.Lu, B.Yang, Biosens. Bioelectron. 2004, 19, 1269-1275.

[212] L. K.Smalls, R.Randall Wickett, M. O.Visscher, Ski. Res. Technol. 2006, 12, 43-49.

[213] S. R.Alonso, P.Ortiz, M.Poll?n, B.P?rez-G?mez, L.S?nchez, M. J.Acu?a, R.Pajares, F. J.Mart?nez-Tello, C. M.Hortelano, M. A.Piris, et al., Am. J. Pathol. 2004, 164, 193-203.

[214] G.Weinlich, W.Bitterlich, V.Mayr, P. O.Fritsch, B.Zelger, Br. J. Dermatol. 2003, 149, 535-541.

[215] J. A.Ramsay, L.From, N. A.Iscoe, H. J.Kahn, J. Invest. Dermatol. 1995, 105, $22-26$.

[216] D. L.Dai, M.Martinka, G.Li, J. Clin. Oncol. 2005, 23, 1473-1482.

[217] T.Vlaykova, L.Talve, M.Hahka-Kemppinen, M.Hernberg, T.Muhonen, Y.Collan, S.Pyrhönen, Oncology 2002, 62, 259-268.

[218] P.Eliopoulos, M. Q.Mohammed, K.Henry, S.Retsas, Melanoma Res. 2002, 12, 139-145.

[219] Y.Wang, D. L.Dai, M.Martinka, G.Li, Clin. Cancer Res. 2007, 13, 4111-4116.

[220] M. M.McCarthy, Clin. Cancer Res. 2006, 12, 3856-3863.

[221] S.Streit, D. S.Mestel, M.Schmidt, A.Ullrich, C.Berking, Br. J. Cancer 2006, 94, 1879-1886.

[222] J.Cruz, J. S.Reis-Filho, P.Silva, J. M.Lopes, Oncology 2003, 65, 72-82.

[223] L. P.Bron, R. A.Scolyer, J. F.Thompson, P.Hersey, Pathology 2004, 36, 561565.

[224] C.Wang, C.Yang, Y.Song, W.Gao, X.Xia, Adv. Funct. Mater. 2005, 15, $1267-$ 1275.

[225] G.McLendon, R.Hake, Chem. Rev. 1992, 92, 481-490.

[226] S.George, H. K.Lee, J. Phys. Chem. B 2009, 113, 15445-15454.

[227] M. V. Mirkin, Allen J. Bard, Scanning Electrochemical Microscopy, Second Edition, CRC Press, 2012.

[228] K.Amreen, A. S.Kumar, Analyst 2016, 141, 2145-2149.

[229] X.Yan, G.Niu, J.Lin, A. J.Jin, H.Hu, Y.Tang, Y.Zhang, A.Wu, J.Lu, S.Zhang, et al., Biomaterials 2015, 42, 94-102.

[230] C.Liang, S.Diao, C.Wang, H.Gong, T.Liu, G.Hong, X.Shi, H.Dai, Z.Liu, Adv. 
Mater. 2014, 26, 5646-5652.

[231] A.Sahu, W.IlChoi, J. H.Lee, G.Tae, Biomaterials 2013, 34, 6239-6248.

[232] X.Zhou, G.Lu, X.Qi, S.Wu, H.Li, F.Boey, H.Zhang, J. Phys. Chem. C 2009, 113, 19119-19122.

[233] A. L.Higginbotham, D.V.Kosynkin, A.Sinitskii, Z.Sun, J. M.Tour, ACS Nano 2010, 4, 2059-2069.

[234] Y.Zhu, D. K.James, J. M.Tour, Adv. Mater. 2012, 24, 4924-4955.

[235] Y. J.Lu, C. W.Lin, H. W.Yang, K. J.Lin, S. P.Wey, C. L.Sun, K. C.Wei, T. C.Yen, C. I.Lin, C. C. M.Ma, et al., Carbon N. Y. 2014, 74, 83-95.

[236] D. A.Jasim, C.Ménard-Moyon, D.Bégin, A.Bianco, K.Kostarelos, J. J. A. Grácio, D.Dash, S.Goel, J.Bean, C. P.Theuer, et al., Chem. Sci. 2015, 6, 3952 3964.

[237] C. J.Omiecinski, J. P.VandenHeuvel, G. H.Perdew, J. M.Peters, Toxicol. Sci. 2011, 120, S49-S75.

[238] T.Cresteil, Food Addit. Contam. 1998, 15, 45-51.

[239] B.Blumberg, W.Sabbagh, H.Juguilon, J.Bolado, C. M.VanMeter, E. S.Ong, R. M.Evans, Genes Dev. 1998, 12, 3195-3205.

[240] S.Mullick Chowdhury, G.Lalwani, K.Zhang, J. Y.Yang, K.Neville, B.Sitharaman, Biomaterials 2013, 34, 283-293.

[241] O.Akhavan, E.Ghaderi, H.Emamy, F.Akhavan, Carbon N. Y. 2013, 54, 419431.

[242] S.Rapino, E.Treossi, V.Palermo, M.Marcaccio, F.Paolucci, F.Zerbetto, Chem. Commun. 2014, 50, 13117-13120.

[243] J.Azevedo, C.Bourdillon, V.Derycke, S.Campidelli, C.Lefrou, R.Cornut, Anal. Chem. 2013, 85, 1812-1818.

[244] G.Eda, G.Fanchini, M.Chhowalla, Nat. Nanotechnol. 2008, 3, 270-274.

[245] M.Luthman, A.Holmgren, Biochemistry 1982, 21, 6628-6633.

[246] X.Li, A. J.Bard, J. Electroanal. Chem. 2009, 628, 35-42.

[247] A. M.Rappaport, Z. J.Borowy, W. M.Lougheed, W. N.Lotto, Anat. Rec. 1954, 119, 11-33.

[248] M.Wachstein, E.Meisel, Am. J. Clin. Pathol. 1957, 27, 13-23.

[249] H. N.Siti, Y.Kamisah, J.Kamsiah, Vascul. Pharmacol. 2015, 71, 40-56.

[250] V.Fuchs-Tarlovsky, Nutrition 2013, 29, 15-21.

[251] B. L.Halvorsen, K.Holte, M. C. W.Myhrstad, I.Barikmo, E.Hvattum, S. F.Remberg, A.-B.Wold, K.Haffner, H.Baugerød, L. F.Andersen, et al., J. Nutr. 2002, 132, 461-471.

[252] K.Wolfe, X.Wu, R. H.Liu, J. Agric. Food Chem. 2003, 51, 609-614.

[253] B.Łata, Sci. Hortic. 2008, 117, 45-52. 
[254] K. E.Hummer, J. F.Hancock, HortScience 2015, 50, 780-783.

[255] K. L.Wolfe, R. H.Liu, J. Agric. Food Chem. 2003, 51, 1676-1683.

[256] B.Łata, J. Agric. Food Chem. 2007, 55, 663-671.

[257] R. H.Liu, M.V.Eberhardt, C. Y.Lee, Nature 2000, 405, 903-904.

[258] C.Guo, J.Yang, J.Wei, Y.Li, J.Xu, Y.Jiang, Nutr. Res. 2003, 23, 1719-1726.

[259] L.Panzella, M.Petriccione, P.Rega, M.Scortichini, A.Napolitano, in Food Chem., 2013, pp. 672-679.

[260] Y. Z.Fang, S.Yang, G.Wu, Nutrition 2002, 18, 872-879.

[261] G.Noctor, C. H.Foyer, Annu. Rev. Plant Physiol. Plant Mol. Biol. 1998, 49, 249-279.

[262] K. W.Lee, Y. J.Kim, D. O.Kim, H. J.Lee, C. Y.Lee, J. Agric. Food Chem. 2003, 51, 6516-6520.

[263] M.Kalinowska, A.Bielawska, H.Lewandowska-Siwkiewicz, W.Priebe, W.Lewandowski, Plant Physiol. Biochem. 2014, 84, 169e188.

[264] F.Shahidi, Y.Zhong, J. Funct. Foods 2015, 18, 757-781.

[265] J. B. L.Tan, Y. Y.Lim, Food Chem. 2015, 172, 814-822.

[266] N.Seawan, W.Vichit, A.Thakam, P.Chaiwut, P.Pintathong, Suranaree J. Sci. Technol. 2014, 21, 301-306.

[267] V.V.Roshchina, J. Fluoresc. 2003, 13, 403-418.

[268] A. A.Karaçelik, M.Küçük, Z.Iskefiyeli, S.Aydemir, S.DeSmet, B.Miserez, P.Sandra, Food Chem. 2015, 175, 106-114.

[269] S. F.Hagen, K. A.Solhaug, G. B.Bengtsson, G. I. A.Borge, W.Bilger, Postharvest Biol. Technol. 2006, 41, 156-163.

[270] D. L.Betemps, J. C.Fachinello, S. P.Galarça, N. M.Portela, D.Remorini, R.Massai, G.Agati, J. Sci. Food Agric. 2012, 92, 1855-1864.

[271] M. N.Merzlyak, A. E.Solovchenko, A. I.Smagin, A. A.Gitelson, J. Plant Physiol. 2005, 162, 151-160.

[272] P.Tacchini, A.Lesch, A.Neequaye, G.Lagger, J.Liu, F.Cortés-Salazar, H. H.Girault, Electroanalysis 2013, 25, 922-930.

[273] M. F.Barroso, N.De-los-Santos-Állvarez, C.Delerue-Matos, M. B. P. P.Oliveira, Biosens. Bioelectron. 2011, 30, 1-12.

[274] H.Li, F.Zhao, L.Yue, S.Li, F.Xiao, Electroanalysis 2016, 28, 1003-1011.

[275] K. Z.Brainina, D. P.Varzakova, E. L.Gerasimova, J. Anal. Chem. 2012, 67, 364-369.

[276] R. de Q.Ferreira, S. J.Greco, M.Delarmelina, K. C.Weber, Electrochim. Acta 2015, 163, 161-166.

[277] I. F. F.Benzie, J. J.Strain, Anal. Biochem. 1996, 239, 70-76.

[278] S.Kuss, D.Trinh, J.Mauzeroll, Anal. Chem. 2015, 87, 8102-8106. 
[279] B.Lertanantawong, A. P.O’Mullane, J.Zhang, W.Surareungchai, M.Somasundrum, A. M.Bond, Anal. Chem. 2008, 80, 6515-6525.

[280] J. L. J.Bezuidenhout, P. J.Robbertse, C.Kaiser, J. Hortic. Sci. Biotechnol. 2005, $80,18-22$.

[281] H.Rymbai, M.Srivastav, R. R.Sharma, S. K.Singh, Sci. Hortic. 2012, 135, 164 170.

[282] L.Pitta Bauermann, W.Schuhmann, A.Schulte, Phys. Chem. Chem. Phys. 2004, 6, 4003.

[283] R.Wang, Z.Wei, W.Wang, Z.Li, Rsc.Org 2012, 1249-1251.

[284] R. K.Mishra, A. M.Vinu Mohan, F.Soto, R.Chrostowski, J.Wang, D.Moatti, G.Reach, J.Phillips, N. A.Monteiro-Riviere, A.Ovsianikov, Analyst 2017, 142, 918-924.

[285] H.Kalluri, A. K.Banga, L.Lewis, J.Agalloco, B.Lambert, R.Madsen, M.Staples, H.Kalluri, A. K.Banga, AAPS PharmSciTech 2011, 12, 431-441.

[286] L.Ventrelli, L.Marsilio Strambini, G.Barillaro, Adv. Healthc. Mater. 2015, 4, 2606-2640.

[287] S.Sharma, A.Saeed, C.Johnson, N.Gadegaard, A. E.Cass, Sens. Bio-Sensing Res. 2017, 13, 104-108. 


\section{Curriculum Vitae}

$\underline{\text { TzU-EN LIN }}$

\section{CONTACT INFORMATION}

MOBILE:+41787668664

TZU-EN.LIN@EPFL.CH

AdDRESS: RUE DE LA MOYA 12, MARTIGNY 1920, SWITZERLAND

\section{PERSONAL INFORMATION}

DATE OF BIRTH : 20, SEPTEMBER, 1987

BIRTH PLACE : TAIPEI, TAIWAN

\section{EDUCATION}

Sep 2013-present

$\mathrm{PhD}$ candidate

Swiss Federal Institute of Technology of Lausanne (EPFL), Dept. of

Chemistry and Chemical Engineering, Switzerland

Feb-Apr, $2017 \quad$ Visiting scholar in Stanford University, USA

Jul 2012 - Sep 2013 Full-time Teaching Assistant, Dept. of Chemistry, National Taiwan

University, Taiwan

Sep 2010 - Jun 2012 National Taiwan University MS, Dept. of Chemistry, Taiwan

Sep 2006 - Jun 2010 National Taiwan University, BS, Dept. of Biochemical Science

Technology, Taiwan

\section{$\underline{\text { AWARDS }}$}

- 2017 Chemistry Travel Award by SCNAT and SCS

- 2017 Stanford-EPFL exchange program scholarship

- $\quad$ ECS 231, New Orleans, Travel grants

- $\quad$ The best poster prize at the 7th European dermatology congress, Spain, 2016

- The 2013 Taiwanese Ministry of Education Technologies Incubation Scholarship

- 2011 LCY CHEMICAL CORP. Scholarship

- Award of Master Thesis Poster

- Member of Phi Tau Phi Scholastic Honor Society

- Dean's Award in BS

- 3 Presidential Awards in BS 


\section{Publications}

1. Electrochemical imaging of cells and tissues. Lin, T.-E.; Rapino, S.; Lesch, A.; Girault, H. H. Chem. Sci. invited paper

2. From tissues to organs: soft electrochemical probes for mapping the distribution of biomarkers and injected nanomaterials in animal and human tissues. Lin, T.-E.; Lu, Y.-J.; Sun, C.-L.; Chen, J.P.; A.; Pick, H.; Lesch, A.; Girault, H. H. Angew. Chem. Int. Ed. Under revision

3. Electrostatic spray ionization from 384-well microtiter plates for mass spectrometry analysis based enzyme assay and drug metabolism screening. Qiao, L.; Zhong, X.; Belghith, E.; Deng, Y.; Lin, T.-E.; Tobolkina, E.; Liu, B.; Girault, H. Anal. Chem. 2017, 89, 895983-5990.

4. Encyclopedia of interfacial chemistry: scanning electrochemical microscopy for bio-imaging. (book chapter) Lin, T.-E.; Bondarenko, A.; Lesch, A.; Girault, H. H. Wiley-VCH in press

5. Mapping the antioxidant activity of apple peels with soft probe scanning electrochemical microscopy. Lin, T.-E.; Lesch, A.; Li, C.-L.; Girault, H. H. J. Electroanal. Chem. 2017, 786, 120-128.

6. Monitoring Tyrosinase Expression in Non-metastatic and Metastatic Melanoma Tissues by Scanning Electrochemical Microscopy. Lin, T.-E.; Bondarenko, A.; Lesch, A.; Pick, H.; CortésSalazar, F.; Girault, H. H. Angew. Chem. Int. Ed. 2016, 55, 3813-3816.

7. Untersuchung der Tyrosinase-Expression in Nicht-metastatischen und Metastatischen Melanomgeweben durch Elektrochemische Rastersondenmikroskopie. Lin. T.-E.; Bondarenko, A.; Lesch, A.; Pick, H.; Cortés-Salazar, F.; Girault, H. H. Angew. Chem. 2016, 11, 3878-3881. (Same article as the previous one)

8. Fixation and Permeabilization Approaches for Scanning Electrochemical Microscopy of Living Cells. Bondarenko, A.; Lin. T.-E.; Petar, S.; Lesch, A.; Cortés-Salazar, F.; Girault, H. H.; Pick, H. Anal. Chem. 2016, 88, 11436-11443.

9. Open-space Microfluidics, Concepts, Implementations, Applications. Lin. T.-E.; Bondarenko, A.; Lesch, A.; Cortés-Salazar, F.; Girault, H. H. Chapter 16\&17. Wiley-VCH in press

10. Multiple Scanning Electrochemical Microscopy Mapping of Tyrosinase in Micro-contact Printed Fruit Samples on Polyvinylidene Fluoride Membrane. Lin, T.-E.; Cortés-Salazar, F.; Lesch, A.; Qiao, L.; Bondarenko, A.; Girault, H. H. Electrochimica Acta 2015, 179, 57-64.

11. Carbon Dots Prepared from Ginger Exhibiting Efficient Inhibition of Human Hepatocellular Carcinoma Cells. Li, C.-L.; Ou, C.-M.; Huang, C.-C.; Wu, W.-C.; Chen, Y.-P.; Lin, T.-E.; Ho, L.-C.; Wang, C.-W.; Shih, C.-C.; Zhou, H.-C.; Lee, Y.-C.; Tzeng, W.-F.; Chiou, T.-J.; Chu, S.-T.; Cang, J.; Chang, H.-T. J. Mater. Chem. B 2014, 2, 4564-4571.

12. Using Protein G-Assembled Magnetic Nanoparticles and Aptamer-Conjugated Gold Nanoparticles to Detect Immunoglobulin G in Plasma Based on Controlled Thrombin Activity. Lin, T.-E.; Li, C.L.; Huang, C.-C.; Chang, H.-T. J. Spectrosc. Dyn. 2014, 4, 9-15.

13. Aptamer-functionalized Nanoparticles for the Detection of Platelet-derived Growth Factor and Immunoglobulin G. Lin, T.-E.; Chang, H.-T. Master Thesis of Dept. of Chemistry, NTU, 2012. 
14. Colorimetric Detection of Platelet-Derived Growth Factors through Competitive Interactions between Proteins and Functional Gold Nanoparticles. Lin, T.-E.; Chen, W.-H.; Shiang, Y.-C.; Huang, C.-C.; Chang, H.-T. Biosens. Bioelectron. 2011, 29, 204-209.

15. Positional Effects on Helical Ala-Based Peptides. Cheng, R. P.; Girinath, P.; Suzuki, Y.; Kuo, H.-T.; Hsu, H.-C.; Wang, W.-R.; Yang, P.-A.; Gullickson, D.; Wu, C.-H.; Koyack, M. J.; Chiu, H.-P.; Weng, Y.J.; Hart, P.; Kokona, B.; Fairman, R.; Lin, T.-E.; Barrett, O.; Biochemistry 2010, 49, 9372-9384. 

